\title{
High viscosity industrial chromatography for mild food fractionation
}

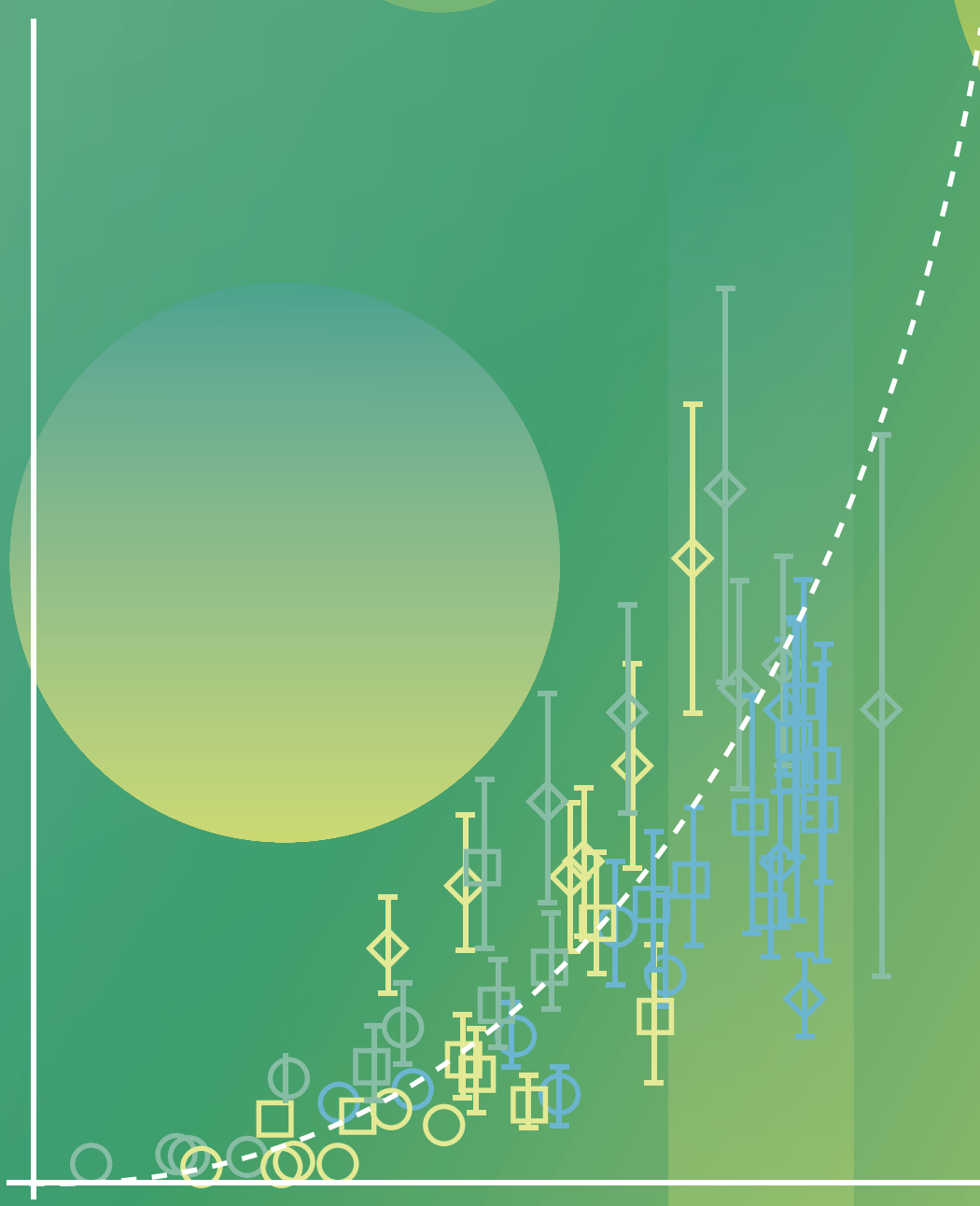

Anton Schultze-Jena 



\section{Propositions}

1. Chromatographic separations become more productive if viscosity is used correctly. (this thesis)

2. A mechanism can be wonderfully counterintuitive despite over half a century of research. (this thesis)

3. The consumer's responsibility towards sustainable consumption is real but limited.

4. Waste is too cheap for people, profit, as well as for the planet.

5. The European Union is the best thing that happened to Europeans since Pizzaro's expedition brought back the potato.

6. Consistency requires creativity.

Propositions belonging to the thesis, entitled

High viscosity industrial chromatography for mild food fractionation

Anton Schultze-Jena

Wageningen, $9^{\text {th }}$ of October, 2019 



\section{High viscosity industrial chromatography for mild food fractionation}

Anton Schultze-Jena 


\section{Thesis committee}

\section{Promotor}

Prof. Dr Albert van der Padt

Special professor Sustainable Production of Food, Wageningen University \& Research

\section{Co-Promotors}

Dr Anja E.M. Janssen

Associate Professor, Food Process Engineering, Wageningen University \& Research

Dr Floor Boon

Scientist, Biobased Products, Wageningen University \& Research

\section{Other members}

Prof. Dr Harry Bitter, Wageningen University \& Research

Prof. Dr Michel Eppink, Wageningen University \& Research

Prof. Dr Hans-Geerd Janssen, Van 't Hoff Institute for Molecular Sciences, University of Amsterdam Prof. Dr Peter de Jong, Van Hall Larenstein University, Leeuwarden

This research was conducted under the auspices of the Graduate School VLAG

(Advanced Studies in Food Technology, Agrobiotechnology, Nutrition, and Health Sciences) 


\title{
High viscosity industrial chromatography for mild food fractionation
}

\author{
Anton Schultze-Jena
}

\section{Thesis}

submitted in fulfilment of the requirements for the degree of doctor

at Wageningen University

by the authority of the Rector Magnificus

Prof. Dr A.P.J. Mol,

in the presence of the

Thesis Committee appointed by the Academic Board

to be defended in public

on Wednesday $9^{\text {th }}$ of October, 2019

at 4.00 p.m. in the Aula. 
Anton Schultze-Jena

High viscosity industrial chromatography for mild food fractionation

140 pages

$\mathrm{PhD}$ thesis, Wageningen University, Wageningen, NL (2019)

With references, with summary in English

ISBN: 978-94-6395-030-5

DOI: $10.18174 / 495572$ 
To my grandmother Moma, who sparks curiosity 



\section{Contents}

Chapter $1 \quad$ Introduction

Chapter 2 The counterintuitive role of extra-column volume in the determination of column efficiency and scaling of chromatographic processes

Chapter 3 Predicting intraparticle diffusivity as function of stationary phase characteristics in preparative chromatograph

Chapter 4 High viscosity preparative chromatography for food applications

Chapter 5 Elevated viscosities in a simulated moving bed using ion-exclusion chromatography: a case study on $\gamma$-aminobutyric acid recovery from tomato serum

Chapter 6 General discussion

Summary

Acknowledgements

About the author

List of publications

Overview of completed training activities 

Chapter 1

\section{Introduction}




\subsection{Introduction}

Agro-materials are a potential source of valuable components (e.g. proteins, peptides, isoflavones, lycopene etc.) or contain reactive molecules like polyphenols that will deteriorate the agro-material. In both cases, these components, often present in low concentration, must be harvested or removed without spoiling the remainder agro-material. Components within a mixture can be separated based on specific characteristics. Membrane processes are used to fractionate components based on size difference, while crystallization processes are used to separate a mixture based on solubility variations. Affinity differences are used as separation mechanism in extraction and chromatographic processes.

Liquid chromatography is commonly used in the pharmaceutical industry to purify and fractionate complex molecules such as proteins and peptides [1]. In the food industry the examples of chromatography are rather scarce, although food industry, along with petro-chemicals, were the first to use countercurrent chromatographic production processes [2]. In the food industry, process streams are generally much larger than in pharmaceutical production, in combination with lower added value products, this results in economically less attractive large equipment. Economic aspects of the separation process can be improved by reducing the size of the chromatographic installation [3].

\subsection{Chromatography}

In a chromatographic system, two different phases are present, the mobile and the stationary phase. The components within the mobile phase are separated based on their variation in affinity for the two phases. The component with the higher affinity for the stationary phase will have a lower migration speed, resulting in a longer retention time. Chromatography is scalable to virtually any size imaginable (millions of tons of product per year [1]), uses limited quantities of resources (i.e. eluent, energy, space, and time) and is a robust process capable of producing active pharmaceutical ingredients under good manufacturing process GMP conditions $[1,2]$.

Different modes of chromatography are defined by their separation mechanism, i.e. size-exclusion, ionexchange, ion-exclusion, hydrophobic interaction, reversed phase, and affinity chromatography [4]. Separation can be achieved by difference in molecular size, ionic charge, solubility, hydrophobicity, affinity, or a combination of these mechanisms. In this thesis mainly size-exclusion chromatography $S E C$ was used. In SEC separation is based on available retention volume for each molecule. Small molecules have a large retention volume, because they can access a large pore volume fraction in the stationary phase. Larger molecules have a smaller retention volume, because they can access only a smaller pore volume fraction, or remain in the interstitial particle volume if they cannot enter the pores at all, and will elute first.

Further, two different kinds of chromatography can be distinguished by their aim, namely analytical and preparative chromatography. The aim of analytical chromatography is to identify and quantify the contents of a sample. Small volumes are used and sample recovery is not necessary, analytes are sometimes modified, denatured, or labelled and discarded as waste after detection [2]. The aim of preparative chromatography is to collect the eluting sample fractions for further use, while maintaining their functionality. Therefore target components are purified and collected, with focus on fast recovery, high productivity, and desired purity. 
Preparative chromatography is a separation method of homogeneous mixtures and can be scaled to input streams from $\mathrm{mL} / \mathrm{min}$ to $\mathrm{m}^{3} / \mathrm{h}$, purifying more than $10 \mathrm{~kg}$ of product per day and $\mathrm{kg}$ of stationary phase material [2]. In the latter case, where columns and installations reach proportions measured in meters and separated products are used on large industrial scale, the separation technique is often called industrial chromatography, in order to emphasize the scale.

\subsection{Design of chromatographic processes}

For the design of an industrial system, chromatographic processes are generally simulated using mathematical models, validated, and optimized at laboratory level and then scaled up to pilot and subsequently industrial scale. These models describe, among other things, the migration of various components in the mobile phase through a column packed with stationary phase and how the column (or columns) are operated. In case of simulated moving bed $S M B$ chromatography, where cycled switching of an array of columns simulates movement of the stationary phase, the switching of the columns is included as design parameter in the model. Generally an optimization routine is set to seek settings that result in highest productivity in combination with constraints given on purity and recovery [5]. Sometimes lowest possible eluent use is included into the optimization goals [6].

Economic feasibility of industrial chromatography requires small and robust separation systems which make use of continuous multicolumn applications, like $S M B$ systems with high productivities. Smaller systems require smaller amounts of stationary phase, which is one of the main contributors to the operation costs [7]. Small systems can be achieved by using chromatographic materials that combine the advantage of being open with small characteristic lengths. The use of open material is even more important to robust operation, when the input feed streams are viscous or turbid. The use of concentrated feed streams leads to a reduction in stream volume and therefore the volumetric flow rate, which is one possible way to reduce system size. Consequently, concentrating product streams by water removal is standard practice is the agro-food industry resulting in highly concentrated and viscous serums, syrups, extracts, etc. with smaller volumes for transport, storage, and various processes. Unfortunately such streams are not acceptable in a chromatographic systems for which they often need to be diluted again to reduce pressure drop and enhance mass transfer.

Another aspect in the design of sustainable and economically feasible chromatographic processes is the use of (relatively pure) water as mobile phase instead of organic solvents or salt. The use of water is cheaper than (organic) solvents, which are sometimes used at smaller scale and in pharmaceutical industries, but it also reduces the number of applicable modes of chromatography. Especially in view of post-process disposal, using water with low levels of contamination is likely to lead to more sustainable processes than water carrying high salt loads from ion-exchange mode or solvents.

Different mathematic models, describing the migration kinetics can be used for the modelling and design of chromatographic processes [8]. The general rate model is the most complex model which requires detailed knowledge on molecular diffusion and adsorption kinetics inside the porous particles, to describe the concentration gradient within the stationary phase particles. The lumped kinetic model is derived from the general rate model by two simplifications: it assumes that both the adsorption-desorption process and the diffusion in the stagnant mobile phase within the pore volume are very fast. In consequence, there is no radial 
concentration gradient within a particle and the percolating mobile phase outside the particles and the stagnant mobile phase inside the pores are constantly in equilibrium. The resistances to mass transfer, inside and outside the particle, are lumped into a single overall mass transfer coefficient, hence the name of the model. Even though the general rate model gives a more detailed outcome, in most cases the lumped kinetic model provides a sufficiently precise approximated result of the behavior of chromatographic columns and are therefore used in this thesis [8].

\subsection{Mass transfer within a chromatographic column}

The behavior of chromatographic columns is governed by thermodynamic, hydrodynamic, and kinetic factors [9]. Thermodynamics describe the interactions of solutes and stationary phase at their equilibrium, hydrodynamics depict the fluid flow inside the column, and kinetics describe how fast solutes move in mobile and stationary phase to reach an equilibrium. All three factors relate how solutes move inside the chromatographic system and it is in the phenomena depicted by these factors, that separation between two components may take place. For successful separation process design, it important to understand and quantify each of these factors.

In size exclusion chromatography, thermodynamic relations display linear isotherms (proportional equilibrium concentrations in stationary and liquid phase). Mass transfer kinetics is generally the most influential parameter governing column behavior in size exclusion chromatography [10]. The individual contributions to mass transfer resistance are eddy dispersion, longitudinal diffusion, film layer mass transfer resistance and the intraparticle diffusivity [11]. These are depicted in Fig. 1.1, taken from [12]. Furthermore, adsorption and desorption kinetics may play a role and friction can influence thermodynamics, but the former is generally assumed to be instantaneous and the latter only plays a role at high or very high pressures, which is only relevant for analytical applications [13]. Both are therefore generally ignored in preparative chromatography.

Axial dispersion is comprised of eddy dispersion and diffusion in longitudinal direction of the column. Eddy dispersion describes the unevenness of flow, how packing and packing structure cause mobile phase in some areas of the column to move faster than in others. This contribution was initially assumed to be independent of velocity [14], but it has been shown, that eddy dispersion is in fact dependent on mobile phase velocity and particle diameter as well as packing quality [1]. The longitudinal diffusion describes the molecular diffusion inside the column along its axial direction. It is only relevant at low velocities and has no significant contribution to overall mass transfer resistance in most preparative chromatographic processes. The external film mass transfer resistance describes the resistance imposed by the stagnant fluid layer surrounding each particle, which needs to be overcome by diffusion [15]. Lastly, trans-particle mass transfer resistance, describes the diffusion of molecules inside the particle. This term is often the main resistance to mass transfer in preparative chromatography $[9,10]$.

The summation of resistances to mass transfer determines how the band of an injected sample moves through the column and how its individual molecules pertain a distribution of migration velocities [16]. The extend of band broadening is also called column efficiency and expressed by the number of plates $N$, which was initially 
defined for Gaussian peak shapes, and later for all possible peak shapes by using the first and second central moment $\mu_{1}$ and $\mu_{2}$ of the elution profile in equation 1.1 [1].

$$
N=\frac{\mu_{1}{ }^{2}}{\mu_{2}}
$$

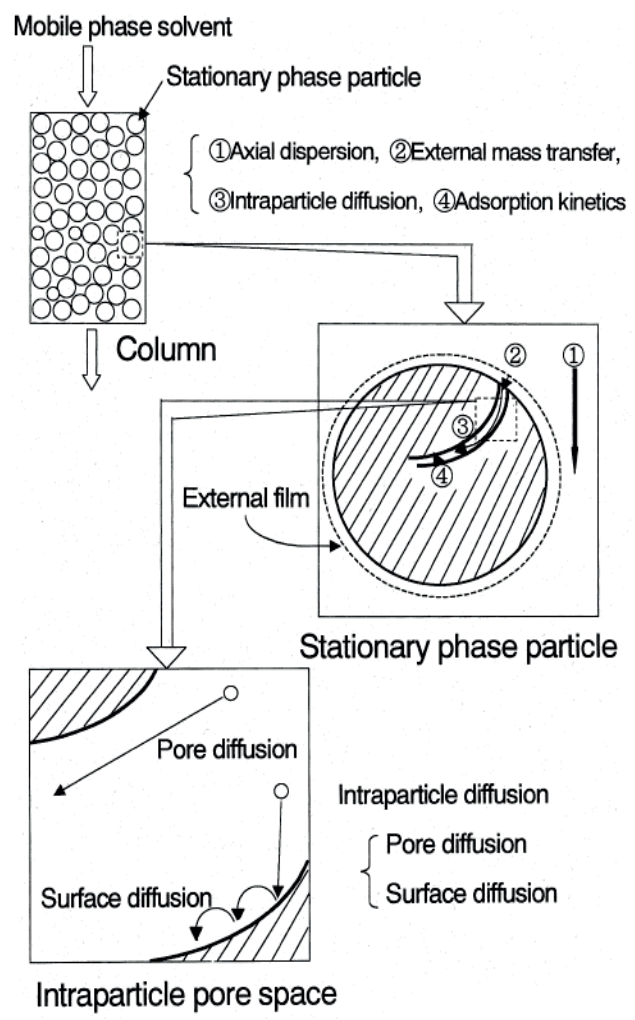

Fig. 1.1 - Illustration of mass transfer inside a column, reproduced from [12].

The height equivalent to a theoretical plate HETP includes the length of the column $L$ and sums up fluid dynamic non-idealities, mass transfer resistances, and ad- and desorption rates in a chromatographic column (equation 1.2) [11, 16].

$$
H E T P=\frac{\mu_{2} \cdot L}{\mu_{1}^{2}}
$$

HETP increases with velocity and is typically plotted in the classic van Deemter curve, illustrated in Fig. 1.2a $[13,17]$. Industrial chromatography is typically operated in the linear region of the curve at relatively high velocities. Fig. 1.2a illustrates the van Deemter curve of two almost identical feed streams under identical conditions, only that stream $A$ has been diluted from stream $B$, resulting in smaller viscosity but higher volume. Fig. 1.2b illustrates the influence velocity and viscosity have on column dimensions. Point 1 in Fig. 1.2a 
operates at low viscosity and velocity, the column length required for the separation is low, but the diameter is large in order to process the large volume at low velocity. Point 2 shows the operation for the same feed stream at higher velocity requires a longer column, as the HETP is increased. Due to the higher velocity, the column diameter is smaller than in point 1 . If the feed stream viscosity is increased, scenario $B$ is applied. Point 3 shows the same HETP as in point 2, but at a lower velocity. Therefore the column length is identical. However, since the volume of stream $B$ is smaller than of $A$, column diameter is decreased. Point 4 shows stream $B$ operating at a velocity equal to point 2 . Column length has to be increased, as HETP is greater in this case. Evaluation of column performance requires knowledge on column efficiency, stream viscosity, and pressure drop over the column bed, when input stream concentration is changed. In this thesis van Deemter curves are used mainly for two different measurements. Intraparticle diffusivity is measured from the slope of the linear region of van Deemter curves in chapter 3, chapter 4, and chapter 5. In chapter 4, the change in slope of van Deemter curves with viscosity is used to find the optimal feed viscosity for productivity.
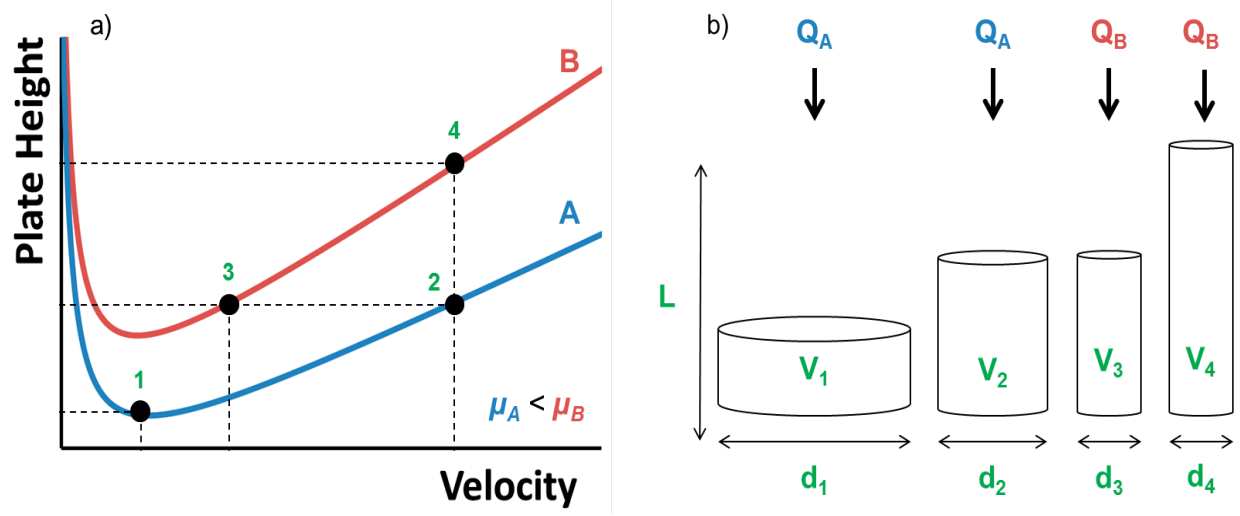

Fig. 1.2 - The interplay between feed viscosity, feed volume, mass transfer and system size, illustrated by a) van Deemter curves of essentially the same feed at different dilution levels $\left(\mu_{A}<\mu_{B}\right)$ and b) the effect different velocities, viscosities and stream volumes have on column dimensions.

\subsection{Chromatography within the food industry}

Examples of current use of chromatography within the food industry are given in Table 1.1. The chromatographic systems process large product streams. A few examples are production of various whey protein isolate products in a continuous simulated moving bed system $S M B$ with a capacity of over 1 million liters of cheese whey per day [18]. A different type of chromatographic system, where the stationary phase is not packed but rather sedimenting down against the upwards flowing feed stream, expanded bed adsorption chromatography, is used for production of lactoferrin, lactoperoxidase, and immunoglobulins from cheese whey, processing more than $200,000 \mathrm{~L}$ of cheese whey per day [18]. In beet sugar processing, sucrose is recovered from molasses by $S M B$ systems with a capacity of over $600 \mathrm{t}$ per day (with $80 \%$ dry matter) [19]. 
Table 1.1 - Examples of chromatographic separations used in food industry. SMB: Simulated moving bed, Batch: single column.

\begin{tabular}{|c|c|c|c|}
\hline $\begin{array}{c}\text { Separation } \\
\text { mechanism }\end{array}$ & Food industry/process & Separation & Process type \\
\hline Ligand exchange & Corn starch refining & Separation of fructose and glucose & $S M B[20]$ \\
\hline Ion exchange & Corn starch refining & Demineralisation & Batch [20] \\
\hline Adsorption & Corn starch refining & Decolorisation & Batch [20] \\
\hline Ion exchange & Beet sugar processing & Softening / decalcification of thin juice & Batch [20] \\
\hline Ion exchange & Beet sugar processing & Recovery of sucrose from molasses & $S M B[20]$ \\
\hline Adsorption & Cane sugar processing & Decolorisation & Batch [20] \\
\hline Ion exchange & Dairy processing & Production of whey protein isolates & Batch, $S M B[18]$ \\
\hline Ion exchange & Dairy processing & Demineralisation of whey & Batch [20], [18] \\
\hline Ion exchange & Dairy processing & $\begin{array}{l}\text { Recovery of lactoferrin, lactoperoxidase } \\
\text { and immunoglobulins from whey }\end{array}$ & Batch, $S M B$ [18], [21] \\
\hline Adsorption & Beverage processing & Debittering of citrus juices & Batch [20], [22] \\
\hline Ion exchange & Beverage processing & Deacidification of fruit juices & Batch [20] \\
\hline Ion exchange & Beverage processing & Demineralisation of grape must & Batch [22] \\
\hline Adsorption & Beverage processing & Protein haze stabilisation & Batch [20] \\
\hline Adsorption & Beverage processing & $\begin{array}{l}\text { Recovery of anthocyanins from grape } \\
\text { juice }\end{array}$ & Batch [22] \\
\hline Ion exchange & Gelatine processing & Demineralisation & Batch [20] \\
\hline Ion exchange & Amino acid production & Recovery L-lysine from fermentation & Unknown [20] \\
\hline
\end{tabular}

Despite these examples, food industry is a minor user of preparative chromatography in comparison to pharmaceutical industry. On the one hand high-value products in pharmaceutical industry provide a wider window of operation of processes than the relatively low-value products in food industry. But on the other hand pharmaceutical industry often relies on production of highly purified ingredients, while in food industry there is a trend towards functional but not necessarily pure fractions [23]. The change of focus away from purity opens opportunities for chromatography within the food industry. In food industry and biorefinery, new process designs emerge, e.g. for obtaining plant proteins from leaves, that focus on developing processes for functional application of existing co-products from the agro-food industry. Van der Goot et al. [23] argue that a conceptual change away from highly purified ingredients towards the refinery of functional fractions will enable the use of more mildly and sustainable processing. Processing towards highly purified fractions in food industry is rarely required. Moreover, it is energy intensive and results in the production of significant waste streams, which often have little value as animal feed or biofuel. Less refined fractions have, in some cases, shown to have more functionality than highly purified fractions [23]. This could be due to functionality loss during processing or synergetic effects in the mixture. This approach of designing chromatographic processes to win food ingredients, needs to balance effects of viscosity with the change in volume, to overcome the main bottle-necks of current process designs. 


\subsection{Aim of this thesis}

Bottle-necks for the separation of the complex/reactive molecules or fractions out of complex food streams are high water usage, complexity and destructiveness of available separation techniques. Consequently, new technologies for the recovery of complex molecules or fractions from large process streams must maintain functionality, use water and energy efficiently, and must be scalable and economical feasible. Chromatography as a technology offers potential to fulfil all these requirements [24].

The aim of this thesis was to contribute to the development of continuous chromatography of agro-streams (plant or dairy based) with high volumes, targeting fractions with (relatively) low value compounds in a food grade environment and in a sustainable manner. The challenge was to separate/fractionate viscous streams in bulk by means of chromatography and to understand the interplay between feed viscosity, mass transfer resistance, pressure drop and eventually productivity and water use of a chromatographic system.

\subsection{Outline of this thesis}

In the design of industrial chromatographic processes, both experiments at lab scale and mathematical model calculations are used. Experiments are performed to determine model parameters and to validate the model. Subsequently the model can be used to scale-up. Experiments require, in addition to the column, the use of further equipment (connectors, tubing, valves, detectors, etc.), all of which add to extra-column volume. The contribution of the extra-column volume to peak broadening is often neglected assuming that by doing so the column efficiency is underestimated and scaling up results in an oversized system. Chapter $\mathbf{2}$ describes how to measure column efficiency and elucidates the importance of extra-column volume and its impact for designing dimensions at industrial scale.

Diffusion inside pores is the rate limiting step in many preparative chromatographic separations and a key parameter for process design. In chapter $\mathbf{3}$ the diffusion of molecules inside porous stationary phases is studied and linked to the properties of both: (small) molecules and stationary phase. Experimental data were related to two existing predictive models from literature.

The implications of using a concentrated feed with an increased viscosity on mass transfer resistance inside a chromatographic column is described in chapter 4. Calculations are made to estimate the effect of viscosity on column volume, while maintaining separation performance (number of theoretical plates) and pressure drop.

In the previous chapters experiments were done with single columns using model solutions with viscosifier agents to increase the viscosity. In chapter 5 a multicolumn separation process $S M B$ is used to obtain a $\gamma$ aminobutyric acid enriched fraction from tomato serum. This separation is based on ion-exclusion chromatography IEC. A mathematical model of the process is validated and used to determine the conditions with the highest productivity.

The thesis concludes with a general discussion in chapter $\mathbf{6}$ on the results in the previous chapters. Further addressed are increasing temperature, as a means to work with concentrated streams while minimizing the viscosity increase, and the influence of a change in peak shape on the separation performance at elevated 
viscosity. Lastly some concluding remarks and future perspective on the chromatography in viscous food applications are given.

\subsection{References}

1. G. Guiochon, D.G. Shirazi, A. Felinger, A.M. Katti, Fundamentals of Preparative and Nonlinear Chromatography, Academic Press, 2006.

2. H. Schmidt-Traub, R. Ditz, Introduction, Preparative Chromatography, Wiley-VCH Verlag GmbH \& Co. KGaA, 2012, pp. 1-6.

3. H. Schmidt-Traub, M. Kaspereit, S. Engell, A. Susanto, A. Epping, A. Jupke, Model-Based Design, Optimization, and Control, Preparative Chromatography, Wiley-VCH Verlag GmbH \& Co. KGaA, 2012, pp. 425-518.

4. O. Coskun, Separation Tecniques: Chromatography, Northern Clinics of Istanbul, (2016).

5. S. Golshan-Shirazi, G. Guiochon, Optimization of experimental conditions in preparative liquid chromatography: Trade-offs between recovery yield and production rate, Journal of Chromatography A, 536 (1991) 57-73.

6. S. Li, Y. Kawajiri, J. Raisch, A. Seidel-Morgenstern, Optimization of simulated moving bed chromatography with fractionation and feedback: Part I. Fractionation of one outlet, Journal of Chromatography A, 1217 (2010) 5337-5348.

7. M. Verzele, Industrial application of preparative liquid chromatography, TrAC Trends in Analytical Chemistry, 6 (1987) 202-205.

8. A. Felinger, G. Guiochon, Comparison of the Kinetic Models of Linear Chromatography, Chromatographia, 60 (2004) pp. 175-180.

9. R.M. Nicoud, Chromatographic Processes, Cambridge University Press, 2015.

10. O. Ludemann-Hombourger, M. Bailly, R.M. Nicoud, Design of a Simulated Moving Bed: Optimal Particle Size of the Stationary Phase, Sep.Sci.Technol., 35 (2000) 1285-1305.

11. A. Seidel-Morgenstern, M. Schulte, A. Epping, H. Schmidt-Traub, M. Schulte, A. SeidelMorgenstern, Fundamentals and General Terminology, Preparative Chromatography, Wiley-VCH Verlag GmbH \& Co. KGaA, 2012, pp. 7-46.

12. K. Miyabe, G. Guiochon, Measurement of the parameters of the mass transfer kinetics in high performance liquid chromatography, Journal of Separation Science, 26 (2003) 155-173.

13. F. Gritti, G. Guiochon, Mass transfer kinetics, band broadening and column efficiency, Journal of Chromatography A, 1221 (2012) 2-40.

14. J.J. van Deemter, F.J. Zuiderweg, A. Klinkenberg, Longitudinal diffusion and resistance to mass transfer as causes of nonideality in chromatography, Frontiers of Chemical Engineering Science, 50 (1995) 3869-3882.

15. F. Gritti, G. Guiochon, A revisit of the concept of external film mass transfer resistance in the packed beds used in high-performance liquid chromatography, Chemical Engineering Science, 72 (2012) 108-114.

16. G. Guiochon, The limits of the separation power of unidimensional column liquid chromatography, Journal of Chromatography A, 1126 (2006) 6-49. 
17. J.J. van Deemter, F.J. Zuiderweg, A. Klinkenberg, Longitudinal diffusion and resistance to mass transfer as causes of nonideality in chromatography, Chemical Engineering Science, 5 (1956) 271289.

18. B.W.K. Woonton, U.; De Silva, K.; Smithers, G.W., Modern Chromatographic Separation Technologies for Isolation of Dairy Ingredients, Advances in Dairy Ingredients, 2013.

19. A.R. Inc., Amalgamated Research Inc. Simulated Moving Bed Chromatography (SMB), in, pp. 3.

20. Dow, Ion exchange and adsorbent solutions for the nutrition market, in, https://www.dow.com/webapps/include/GetDoc.aspx?filepath=liquidseps/pdfs/noreg/17703529.pdf, 2017.

21. M. Tomita, New Technologies and their Application in the Milk Industry, Asian Australasian Journal of Animal Sciences, 13 (2000) 376-382.

22. Resindion, Ion exchange resins, adsorbent resins, and chromatographic resins in food industry, in, pp. 28.

23. A.J.v.d. Goot, P.J.M. Pelgrom, J.A.M. Berghout, M.E.J. Geerts, L. Jankowiak, N.A. Hardt, J. Keijer, M.A.I. Schutyser, C.V. Nikiforidis, R.M. Boom, Concepts for further sustainable production of foods, Journal of Food Engineering 168 (2016), (2016).

24. H. Schmidt-Traub, M. Schulte, A. Seidel-Morgenstern, Preparative Chromatography, Wiley, 2012. 


\section{Chapter 2}

\section{The counterintuitive role of extra-column volume in the determination of column efficiency and scaling of chromatographic processes}

This chapter has been published as:

A. Schultze-Jena, M.A. Boon, P.J.T. Bussmann, A.E.M. Janssen, A. van der Padt, The counterintuitive role of extra-column volume in the determination of column efficiency and scaling of chromatographic processes, Journal of Chromatography A, 1493 (2017) 4956. 


\section{Abstract}

In industrial liquid separation processes chromatography often has a key function in the optimization of yield and purity. For the design of an industrial system, chromatographic processes are generally simulated using mathematical models, tested and optimized at laboratory level, and then scaled up to pilot and subsequently industrial scale. To describe the system, experimental data and model data need to be fitted and extra column contribution must be determined. This paper describes the influence of extra-column volume on overall separation efficiency for lab scale and its impact on the design of large scale systems.

Measurement of extra-column contribution was investigated in terms of mean retention time and variance using two different methods: the commonly used zero dead volume connector and as an alternative the zero length column. Further, a technique is presented to estimate extra-column contribution to band broadening for different injection volumes, velocities, and tracers based on representative measurements.

When scaling up, often contribution of extra-column volume from laboratory equipment is neglected assuming to be on the safe side, however column efficiency is often lower than efficiency measured for the entire chromatographic system. Relation between system efficiency and column efficiency was investigated using laboratory data and the lumped kinetic model. Depending on the ratio of extra-column volume to retention volume in the system, deduced column efficiency was up to $20 \%$ smaller than overall system efficiency. This ratio revealed the misleading nature of the term efficiency loss, when describing influence of extra-column volume on column efficiency. A scheme, which relates the relative variance of the system to the relative extra-column volume, provided an assessment of under- or overestimation of column efficiency. In this article it is shown how scaling up a system based on laboratory data, where extra-column volume contribution is not accounted for, may severely overestimate column efficiency. This overestimation results in underestimated column dimensions at pilot and industrial scale, and hence underperformance of the industrial system. 


\subsection{Introduction}

Chromatographic separation methods are common practice in most analytical and preparative separation applications. Except for thin layer chromatography, all chromatographic methods share inherent construction of one or several columns connected to equipment such as detectors and valves via tubing/piping and connectors. Scaling up chromatographic processes starts with the right interpretation of laboratory experiments. When analyzing any chromatogram it is important to keep in mind that mobile phase and analytes pass not only through the column but through the entire system, every part of equipment in the flow path between sample injection and detection including the column. Each part adds to overall retention time and band broadening (expressed in terms of variance). If the column is taken out, then the resulting chromatogram shows the extra-column contribution to retention time and variance [1-3]. It is not possible to show the chromatogram of the column alone. Only by accounting for extra-column contribution in the measured system, properties of the column can be deduced. If the contribution to band broadening of the chromatographic column is not separated from the contribution of the extra-column volume, then estimation of column dimensions during development and scaling up may be subject to large errors.

The challenge of determining the influence of extra-column volume to band broadening and role of individual equipment parts before and after the column was first discussed in 1966 for gas chromatography by Sternberg [1]. The concept of extra-column band broadening was theoretically applied to analytical liquid chromatography in 1975 [4] and practically investigated the following year for contribution of injection system, detectors, connectors and guard columns [2]. These articles demonstrated an increase in peak variance caused by equipment parts before and after the column and argued for a minimization of extra-column band broadening in order to utilize the full separation efficiency of the column. More recent literature was primarily concerned with minimization of extra-column volume and optimization of flow channels to reduce extracolumn variance [2, 5-7] as well as modelling the effect of each part [6, 8-11].

Column efficiency, describing band broadening of an injected pulse migrating along the column, is one of the most important scaling parameters [12]. When scaling up a system, column efficiency should be kept constant [13] or must be accounted for. Several publications stated that efficiency of the column was almost or always higher than efficiency of the entire system $[8,14,15]$. Most publications reviewed for this work did not specify retention time and variance of column and of extra-column volume, so the reader cannot deduce column performance within that specific system e.g. $[6,15,16]$. Preliminary experiments indicated that efficiency, defined on the basis of retention time and variance, was not always reduced when extra-column contribution was taken into account. A system efficiency larger than the column efficiency has to our knowledge not been described before. It opposes the intuitive term "efficiency loss", which is associated with extra-column volume [5].

This work aimed at better understanding efficiency loss due to extra-column volume and extra-column contribution to band broadening. Elution profiles of pulse injections were investigated for the calculated efficiency before and after accounting for extra-column volume, and the resulting efficiency loss was analyzed. Two methods for measurement of extra-column contribution were compared: a zero dead volume connector $Z D V$ and the alternative "zero length column". The latter retained all construction parts found in the resin filled column (flow distributors, frits, filters, etc.), except for the space the resin occupied in a regular column as well as the resin itself, and therefore gave a more accurate representation of extra-column 
contribution to retention time and variance than the commonly used $Z D V$. In addition, the influence of injection volume, mobile phase velocity and tracer molecule on extra-column contribution was investigated to enable accurate estimation of extra-column variance for different experimental settings. This work shows that in many cases system efficiency is higher than column efficiency after correction for extra-column contribution.

A guide to estimate under- or overestimation of column efficiency when extra-column contribution is not taken into account is provided in the last chapter. When scaling up a column based on efficiency calculated for the system, column dimension could be either over- or underestimated.

\subsection{Theoretical background - column efficiency}

Discussion on column efficiency began in a time when chromatography was still considered to be a succession of discontinuous equilibration steps with a step height and a specific time to achieve equilibration analogue to a classical distillation column [12]. Chromatography is now understood as a continuous process, but the idea of plates and plate height still holds and the number of plates $N$ in a column serves as a dimensionless number for column efficiency, as explained in detail in an excellent review by Guiochon [12]. Column length $L$ divided by $N$ gives the height equivalent of the theoretical plate HETP, which describes retention time distribution of the same molecular species within a sample moving through the column $[3,12]$.

For interpretation of column performance, measured data of the system has to be corrected for influence of extra-column volume. $N$ is calculated as ratio of squared mean retention time $\bar{t}_{R}^{2}$ over peak variance $\sigma^{2}$. Analyzing a chromatogram yields $\bar{t}_{R . S y s t e m}^{2}$ and $\sigma_{\text {System }}^{2}$ which are used to calculate $N_{\text {System }}$. Assuming that individual contributions to peak broadening are independent of each other, column efficiency $N_{\text {Column }}$ is calculated as shown in equation $2.1[3,4]$ :

$$
N_{\text {Column }}=\frac{\left(\bar{t}_{\text {R.System }}-\bar{t}_{\text {R.ECC }}\right)^{2}}{\sigma_{\text {System }}^{2}-\sigma_{\text {ECC }}^{2}}=\frac{\bar{t}_{R . \text { Column }}^{2}}{\sigma_{\text {Column }}^{2}}
$$

where $\sigma_{E C C}^{2}$ is peak variance and $\bar{t}_{R . E C C}$ mean retention time of extra-column contribution. Through this subtraction of extra-column contribution from system data, influence of all construction parts of the extracolumn volume, but also the inherent variance to the injection volume, are accounted for. As previously mentioned the reduction of $N_{\text {Column }}$ to $N_{\text {System }}$ is described as efficiency loss [8, 14, 15], meaning the loss of system efficiency due to extra-column contribution. In terms of retention times and variances, efficiency loss is described by equation 2.2 :

$$
\begin{aligned}
& N_{\text {Column }}>N_{\text {System }} \Rightarrow \frac{\bar{t}_{\text {R.Column }}^{2}}{\sigma^{2} \text { Column }}>\frac{\bar{t}_{\text {R.System }}^{2}}{\sigma_{\text {System }}^{2}} \\
& \Rightarrow\left(\frac{\bar{t}_{\text {R.System }}-\bar{t}_{R . E C C}}{\bar{t}_{\text {R.System }}}\right)^{2}>\frac{\sigma_{\text {System }}^{2}-\sigma_{\text {ECC }}^{2}}{\sigma_{\text {System }}^{2}}
\end{aligned}
$$


Rearranging equation 2.2 shows, if the ratio of variances is smaller than the ratio of squared mean retention times, $N_{\text {Column }}$ is greater than $N_{\text {System }}$.

\subsection{Materials and methods}

\subsubsection{Materials}

\section{Chemicals}

All experiments used Milli-Q water as mobile phase. Tracers were $\mathrm{D}_{2} \mathrm{O}$, glucose, urea, and dextran T2000 (all four obtained from Sigma Aldrich, St. Louis, MO, USA). $\mathrm{D}_{2} \mathrm{O}$ was used pure and in 1:5 dilution in Milli-Q water when $1 \mathrm{~mL}$ sample volume was applied. Dextran $(3 \mathrm{~g} / \mathrm{L}$ ), urea, and glucose (both $5 \mathrm{~g} / \mathrm{L}$ ) were dissolved in Milli-Q water. The column was packed with Dowex Monosphere $99 \mathrm{Ca} / 320$ polystyrene resin (Supelco, Bellefonte, PA, USA), mean particle diameter $307 \mu \mathrm{m}$, measured with a Mastersizer 2000 (Malvern, UK).

\section{Instruments}

For high-pressure liquid chromatography a Wellchrom set-up with a K-1001 pump and a K-2401 RI-detector was used, all from Knauer, Germany. Further a Julabo F25 MP controlled temperature in the column jacket and a mini Cori-Flow flowmeter (Bronkhorst, The Netherlands) measured flow rate after the detector. All components were connected with 0.02" PEEK tubing (Grace, Deerfield, IL, USA).

\section{Column}

Column measurements were made on a slurry packed Götec Superformance 300-10 column (300 x 10 mm) with tefzel capillaries of $35 \mathrm{~cm}$ lengths and ID $0.5 \mathrm{~mm}$ (Götec, Germany), including flow adapter with frits and filter. Bed height was consistent around $24-25 \mathrm{~cm}$. Before use, resin counter-ion was exchanged from $\mathrm{Ca}^{2+}$ to $\mathrm{K}^{+}$with $5 \mathrm{CV}$ 's of $1 \mathrm{M} \mathrm{KCl}$ (Sigma Aldrich, St. Louis, MO, USA) in Milli-Q water and the column was re-packed after re-swelling in Milli-Q water. The zero length column was made from a Götec Superformance $10-10$ column $(10 \times 10 \mathrm{~mm})$. External porosity $(38.6 \%)$ was measured with $3 \mathrm{~g} / \mathrm{L}$ dextran (average molecular weight of approximately 2,000,000 Da) and the total porosity (77.7\%) measured with $\mathrm{D}_{2} \mathrm{O}$.

\subsubsection{Methods}

\section{Chromatographic analysis}

All chromatographic measurements were conducted as pulse injections, varying tracer, injection volume, and velocity. The column was kept at $25^{\circ} \mathrm{C}$ through a water jacket. Elution peaks exhibited different shapes and degrees of asymmetry which rendered calculation of central moments the most accurate method to determine mean retention time and variance $[17,18]$. In Excel the $0^{\text {th }}$ central moment $\mu_{0}, 1^{\text {st }}$ central moment $\mu_{1}$, and $2^{\text {nd }}$ central moment $\mu_{2}$ were determined as described in [19]. Integration limits were set manually and baseline drift was corrected for manually, where necessary, to mitigate common concerns of inaccuracy when using the method of moments $[14,20,21]$. $N_{\text {System }}$ was calculated from $\mu_{1}$ and $\mu_{2}$ with equation 2.3. HETP $P_{\text {System }}$ was calculated from $\mu_{1}, \mu_{2}$, and $L$ with equation 2.4. 


$$
\begin{aligned}
& N_{\text {System }}=\frac{\mu_{1}^{2}}{\mu_{2}}=\frac{\bar{t}_{R . \text { System }}^{2}}{\sigma^{2} \text { system }} \\
& \text { HETP }_{\text {System }}=L \frac{\mu_{2}}{\mu_{1}^{2}}=L \frac{\sigma^{2} \text { System }}{\bar{t}_{\text {R.System }}^{2}}
\end{aligned}
$$

Mean retention time $\bar{t}_{R}$ was converted to retention volume $V_{R}$ by multiplying $\bar{t}_{R}$ with volumetric flow rate $Q_{V}$. Variance $\sigma^{2}$ from time units $\left(\mathrm{s}^{2}\right)$ was converted into volumetric units $\left(\mathrm{mL}^{2}\right)$ by multiplying with $Q_{V}{ }^{2}$. The linear superficial velocities $u_{S}$ were $1,2.5,5$, or $7.5 \mathrm{~m} / \mathrm{h}$.

\section{Measurement of extra-column contribution to peak broadening}

Extra-column contribution to peak broadening, in terms of mean retention time and variance, can be measured when the column is replaced by a suitable connection. Most commonly the column is replaced with a zero dead volume connector $Z D V$ which connects column input directly to column output tubing [17]. Using a $Z D V$ important parts inherent to most preparative columns such as flow adapters, filters, and fixed column tubing are bypassed, resulting in a different extra-column volume and flow path. An alternative method, with the column in place, requires measurement of several tracers with a large range in retention factors [22, 23]. This method is not feasible for all chromatographic separations, such as separations based on size exclusion.

In this study an alternative method of extra-column contribution measurement, the "zero length column", was compared to the use of a $Z D V$. The zero length column featured all construction details of the resin packed column used in column experiments. The flow adapters, however, were positioned to touch each other, leaving no space for resin in between. The concept of the zero length column is also used for the determination of sorption kinetics with small amounts of resin, e.g. [24]. The zero length column used in this study is similar in construction, except that it does not contain any resin. Mean retention volume in extra-column measurements changes with linear velocity, due to changes in dimensions of the stagnant layer volume. To account for this effect, the extra-column volume was determined at room temperature for each tracer by extrapolating mean retention times of pulse injections in extra-column volume for different flow rates to $0 \mathrm{~mL} / \mathrm{min}$; as described in [17].

For considerations of extra-column contribution in chapters 2.4.1 to 2.4.3, the volumetric flow rate $Q_{V}$ was used for display and discussion $(1.3,3.4,6.7$, and $9.9 \mathrm{~mL} / \mathrm{min})$ rather than the linear superficial velocity $u_{S}$, since the mobile phase in the extra-column volume passes through several parts with different geometries.

\section{Injection Volume}

Injection volumes were varied with different volume sample loops in between $40 \mu \mathrm{L}$ and $1 \mathrm{~mL}$. Influence of sample volume $V_{I n j}$ on variance $\sigma_{I n j}^{2}$ was calculated with equation 2.5 as described in [1, 4, 5, 25-27] with dimensionless parameter $D^{2}=12$ for rectangular injection [1].

$$
\sigma_{I n j}^{2}=\frac{V_{I n j}^{2}}{D^{2}}
$$




\section{Bulk diffusion coefficients}

The bulk diffusion coefficients $D_{M}$ of tracers in water were taken from literature sources: $\mathrm{D}_{2} \mathrm{O} 2.1 \cdot 10^{-9}$ [28], dextran $1.310^{-11}$ [29], glucose $6.8 \cdot 10^{-10}[30]$, and urea $1.2 \cdot 10^{-9}[31]\left(\right.$ all in $\left.\mathrm{m}^{2} / \mathrm{s}\right)$.

\section{Simulating elution profiles}

Experimental research was extended beyond laboratory boundaries of limited column length and retention of size exclusion chromatography, by modelling elution peaks with the lumped kinetic model as described by [32] using gProms Model builder 4.2.0. $\mathrm{D}_{2} \mathrm{O}$ elution peaks were simulated for a constant linear superficial velocity $\left(u_{S}=1 \mathrm{~m} / \mathrm{h}\right)$ with increasing retention factor $\left(k^{\prime}\right)$ of 0.65 through 4.13 and increasing bed height $(0.1$ through $5 \mathrm{~m}$ ). The resulting chromatograms were analyzed with the method of moments. The lumped kinetic model only describes the column; for estimation of system data, extra-column contribution measured experimentally with the zero length column was added, analog to equation 2.1. Input parameters for the lumped kinetic model were calculated according to $[18,32]$ (Table 2.1). Particle diffusivity across the porous stationary phase $D_{P}$ was fitted to experimental data.

Table 2.1 - Input parameters for lumped kinetic model, respective values and units. Calculated according to references [18, 32].

\begin{tabular}{r|ll} 
Input variable & Value & Unit \\
\hline Concentration & 500 & $\mathrm{~kg} / \mathrm{m}^{3}$ \\
Equilibrium constant $\mathrm{k}^{\prime}$ & 0.65 & - \\
Lumped distribution coefficient $\mathrm{k}_{\mathrm{f}}$ & 0.17 & $1 / \mathrm{s}$ \\
Zone retention factor $\mathrm{k}_{1}$ & 1.02 & - \\
Film transfer coefficient $\mathrm{k}_{\mathrm{f}}$ & $9.2 \cdot 10^{-5}$ & $\mathrm{~m} / \mathrm{s}$ \\
Axial dispersion $\mathrm{D}_{\mathrm{L}}$ & $3.5 \cdot 10^{-8}$ & $\mathrm{~m}^{2} / \mathrm{s}$ \\
Particle diffusivity $\mathrm{D}_{\mathrm{P}}($ fitted $)$ & $2.9 \cdot 10^{-10}$ & $\mathrm{~m}^{2} / \mathrm{s}$
\end{tabular}

\subsection{Results and discussion}

\subsubsection{Measuring extra-column contribution to variance and retention volume}

The extra-column contribution to variance and retention volume was measured with two methods: a zero dead volume connector $Z D V$ and an alternative method, the zero length column. Extra-column volume was $20 \%$ smaller when measured with the $Z D V$ compared to the zero length column. Difference in variance was nearly constant, roughly $0.01 \mathrm{~mL}^{2}$ smaller, when measured with the $Z D V$ compared to the zero length column. The variance difference was independent on injected volume, employed flow rate and tracer (Fig. 2.1). The majority of variance could therefore be attributed to system parts identical in zero length column and $Z D V$ such as tubing, valves, and detector. We expect the constant difference between peak variances measured with $Z D V$ and zero length column to be general and independent of the chromatographic unit. We expect that the actual value and constant difference is specific for the $Z D V$ and zero length column used. Measured mean retention times and variances for all four tracers and injection volumes of $200 \mu \mathrm{L}$ are listed in Table 2.2. 


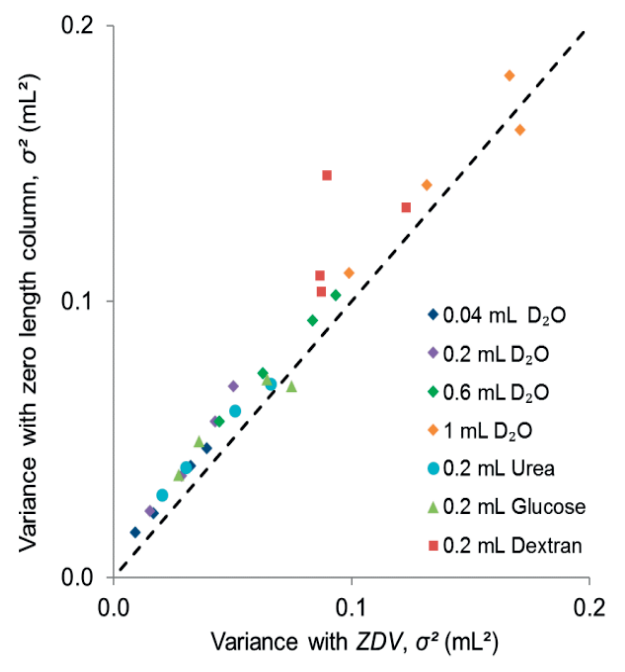

Fig. 2.1 - Variances measured with zero length column over variances measured with $Z D V$ connector for different injection volumes $(0.04$ through $1 \mathrm{~mL})$, volumetric flow rates (1.3 through $10 \mathrm{~mL} / \mathrm{min})$, and tracers $\left(\mathrm{D}_{2} \mathrm{O}\right.$, dextran, glucose, and urea).

Table 2.2- Measured mean retention time $\mu_{1}$ and peak variance $\mu_{2}$ in column, zero length column and zero dead volume connector $Z D V$ for all four tracers at four different velocities. All injection volumes were $200 \mu \mathrm{L}$.

\begin{tabular}{cc|cccccc} 
Molecule & Velocity & \multicolumn{2}{c}{ Column } & \multicolumn{2}{c}{ Zero length column } & \multicolumn{2}{c}{ ZDV } \\
\hline \multirow{5}{*}{$D_{2} O$} & $u_{S}(\mathrm{~m} / \mathrm{h})$ & $\mu_{1}(\mathrm{~mL})$ & $\mu_{2}\left(\mathrm{~mL}^{2}\right)$ & $\mu_{1}(\mathrm{~mL})$ & $\mu_{2}\left(\mathrm{~mL}^{2}\right)$ & $\mu_{1}(\mathrm{~mL})$ & $\mu_{2}\left(\mathrm{~mL}^{2}\right)$ \\
& 1.01 & 17.12 & 1.61 & 1.21 & 0.02 & 0.98 & 0.02 \\
& 2.59 & 17.18 & 3.34 & 1.32 & 0.04 & 1.05 & 0.03 \\
& 5.13 & 17.07 & 4.86 & 1.39 & 0.06 & 1.13 & 0.04 \\
& 7.60 & 17.33 & 6.49 & 1.43 & 0.07 & 1.26 & 0.05 \\
Urea & 1.01 & 18.82 & 4.25 & 1.22 & 0.03 & 0.99 & 0.02 \\
& 2.59 & 19.08 & 9.78 & 1.31 & 0.04 & 1.09 & 0.03 \\
& 5.13 & 19.52 & 18.78 & 1.47 & 0.06 & 1.20 & 0.05 \\
& 7.60 & 19.88 & 24.84 & 1.50 & 0.07 & 1.18 & 0.07 \\
Glucose & 1.01 & 14.41 & 5.72 & 1.25 & 0.04 & 1.01 & 0.03 \\
& 2.59 & 14.51 & 14.26 & 1.31 & 0.05 & 1.09 & 0.04 \\
& 5.13 & 14.82 & 23.84 & 1.42 & 0.07 & 1.24 & 0.07 \\
& 7.60 & 13.83 & 19.66 & 1.45 & 0.07 & 1.26 & 0.06 \\
& 1.01 & 8.92 & 0.31 & 1.26 & 0.11 & 1.09 & 0.09 \\
& 2.59 & 8.94 & 0.35 & 1.36 & 0.10 & 1.13 & 0.09 \\
& 5.13 & 9.31 & 0.41 & 1.48 & 0.15 & 1.25 & 0.09 \\
& 7.60 & 9.32 & 0.42 & 1.57 & 0.13 & 1.21 & 0.12
\end{tabular}


Suitability of the use of $Z D V$ to determine the extra-column contribution to variance was also studied by other authors. Gritti \& Guiochon for example concluded that measurement with the $Z D V$ yields results slightly smaller than the extra-column contribution measured with other, more elaborate methods [12,23]. The authors further go on to label the difference to be small and of little consequence [17], unless working at pressures of several hundred bars [8]. In the system measured here, deduced column efficiency was about $3 \%$ smaller, when extra-column contribution was measured with the zero length column compared to measurement with the $Z D V$ connector. This, albeit small, difference is attributed to tubing, flow distributers, filters, and frits, which are measured with the zero length column, but omitted when measuring with the $Z D V$. Although the difference is small, we advise to use the zero length column when the extra-column contribution to retention time and variance needs to be determined with accuracy. For all following observations, the zero length column was used for measurements of extra-column contribution.

\subsubsection{Injection volume influence on extra-column contribution}

A pulse of tracer injected into the system differs from the ideal Dirac function that is assumed in theory [21]. Injection volume and volumetric flow rate of the mobile phase contribute to mean retention time and variance and therefore to overall measured mean retention time and variance $[1,12]$. With increasing injection volume, measured variance of $\mathrm{D}_{2} \mathrm{O}$ pulse injections increased at each measured flow rate (Fig. 2.2). The variance of a sample $\sigma_{I n j}^{2}$, ideally represented by a rectangular function when injected via an injection loop, was calculated with equation 2.5. Subtraction of $\sigma_{I n j}^{2}$ from the total variance measured with the zero length column $\sigma_{E C C}^{2}$ led to the residual variance caused by extra-column volume. The calculated residual variances spread increasingly with greater volumetric flow rate (open symbols in Fig. 2.3) indicating a dependence of $\sigma_{I n j}^{2}$ on volumetric flow. Therefore the parameter $D^{2}$ from equation 2.5 was fitted to minimize the standard deviation between the residual variances within each flow rate (closed symbols in Fig. 2.3). With this fitted parameter the relative standard deviation between residual variances was reduced from around $26 \%$ with $D^{2}=12$ to $12 \%$. Measured mean retention times and variances for all four tracers and injection volumes of $200 \mu \mathrm{L}$ are listed in Table 2.2 .

Resulting values for the fitted $D^{2}$ were plotted over volumetric flow rate (Fig. 2.4). At a flow rate of zero there was no deviation from plug flow and therefore $D^{2}=12$ [1]. As flow rate increased, the injection profile differed more from a rectangular shape and attributed increasingly to the variance of the injection volume and as a result $D^{2}$ decreased. This relation has to our knowledge not been described before; $D^{2}$ has rather been described as a function of injection volume [22] and independent of velocity [1, 4, 5]. These results were confirmed by applying the same analysis on data from literature [22] (Fig. 2.9, supplementary material). It is therefore concluded that it is possible to estimate extra-column contribution to variance independent of injection volume and at different flow rates with reasonable accuracy. For a given experimental set-up, this enables the estimation of extra-column contribution for different injection volumes and mobile phase flow rates from a single measurement. However, $\sigma_{E C C}^{2}$ is not equal to the variance of a band profile that enters the column; for the distinction between pre- and post-column band broadening, further analytical techniques are required [11]. 


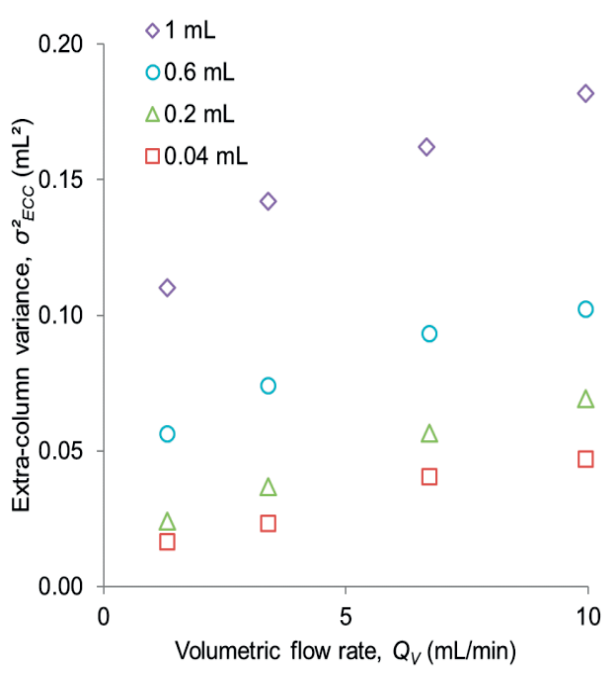

Fig. 2.2 - Extra-column variances at increasing volumetric flow rates for $\mathrm{D}_{2} \mathrm{O}$ injection volumes of 0.04 , $0.2,0.6$, and $1 \mathrm{~mL}$.

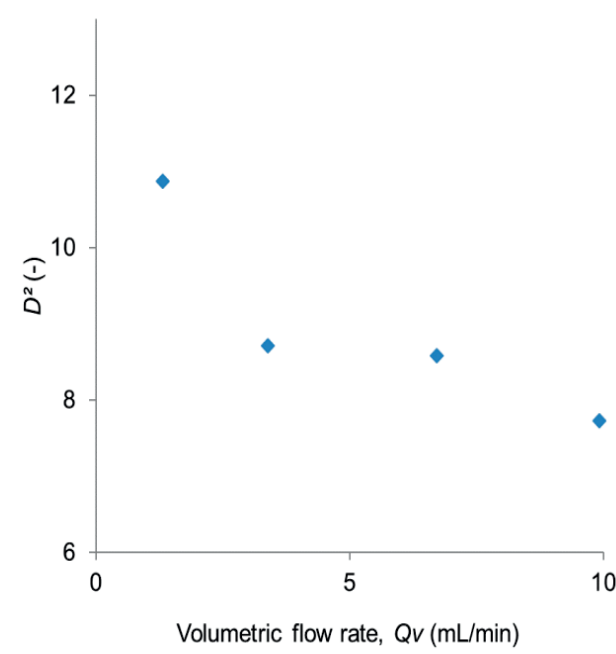

Fig. 2.4 - Fitted dimensionless parameter $D^{2}$ over volumetric flow rate $Q_{V}$ to estimate variance contribution of injection volume. Fitted to minimize standard deviation between measured peak variances (Fig. 2.3).

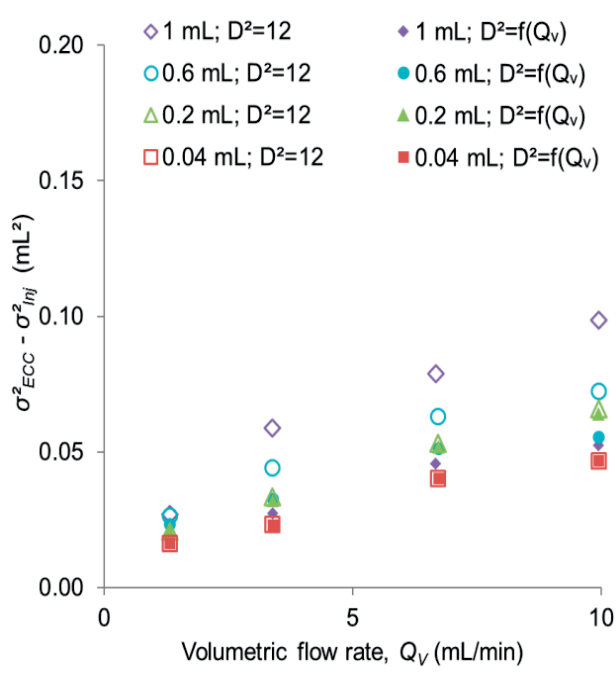

Fig. 2.3 - Variances of injection volume $\sigma_{I n j}^{2}$ subtracted from measured peak variances $\sigma_{E C C}^{2}$ at increasing volumetric flow rates for $\mathrm{D}_{2} \mathrm{O}$ injection volumes of 0.04 , $0.2,0.6$, and $1 \mathrm{~mL} \cdot \sigma_{I n j}^{2}$ calculated with $\mathrm{D}^{2}=12$ (open symbols), as suggested in literature, and $D^{2}$ fitted as a function of flow rate (closed symbols). 


\subsubsection{Tracer molecule influence on extra-column contribution}

Band broadening inside extra-column volume is governed by the mobile phase flow profile and tracer diffusivity [17]. Two main diffusive directions are prevalent inside extra-column volume: radial and longitudinal. Diffusive transport will be far smaller than convective transport in the same direction. Although molecules diffuse against the direction of convective flow as well, the short retention time in the extra-column volume limits the influence on peak variance; therefore longitudinal diffusivity can be neglected. Diffusion in radial direction however may relax parabolic flow profiles traveling through tubing [8] and therefore influence measured peak variance. To study influence of diffusivity on peak variance, pulse injections of $\mathrm{D}_{2} \mathrm{O}$, glucose, urea, and dextran at various flow rates were analyzed.

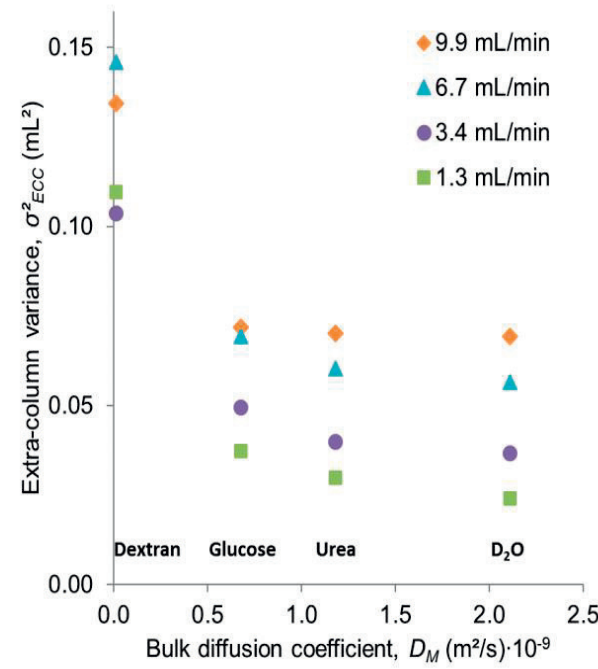

Fig. 2.5 - Variance of extra-column volume over bulk diffusion coefficient $D_{M}$, measured for pulse injections $(200 \mu \mathrm{L})$ of different tracer molecules at different volumetric flow rates.

Upon increasing flow rate, larger peak variances were measured for all tracer molecules, with exception of dextran (Fig. 2.5). With smaller bulk diffusion coefficient $D_{M}$, greater peak variances were measured at all flow rates, indicating less relaxation of the parabolic flow profile through radial diffusion. The relative standard deviation between peak variances measured for $\mathrm{D}_{2} \mathrm{O}$, glucose, and urea was $18 \%, 13 \%, 9 \%$, and $2 \%$ at flow rates of 1.3, 3.4, 6.7 and $9.9 \mathrm{~mL} / \mathrm{min}$ respectively. Extra-column contribution to band broadening should be measured under the same experimental conditions used for column operation [17]. For process design purposes this should include use of actual target molecules as tracer as well as actual feed as mobile phase. The feed composition is relevant when it influences the flow profile, diffusivity and interaction with the stationary phase. Based on data shown here extra-column contribution to variance for tracers with similar diffusivities could be estimated. As the diffusivity of the tracer influences the measured peak variance less at greater velocities, the estimation will be more accurate the higher the velocity of the mobile phase. Measured mean retention times and variances for all four tracers and injection volumes of $200 \mu \mathrm{L}$ are listed in Table 2.2 . 


\subsubsection{Column efficiency compared to system efficiency}

Influence of extra-column contribution on system efficiency became apparent when column efficiency $N_{\text {Column }}$ was compared to system efficiency $N_{\text {System }}$. As shown in equation 2.2, $N_{\text {Column }}$ is greater than $N_{\text {System }}$ when the ratio of variances is smaller than the ratio of retention times. Plotting experimental ratios of variance against ratios of retention time for $\mathrm{D}_{2} \mathrm{O}$, glucose, and urea however, showed $N_{\text {Column }}$ smaller than $N_{\text {System }}$ (closed symbols in Fig. 2.6). With increasing velocity, ratios of $N_{\text {System }}$ over $N_{\text {Column }}$ became greater, as indicated by the top left arrow. The same behavior was found irrespective of injection volume and flow rate, only for dextran was $N_{\text {Column }}$ consistently greater than $N_{\text {System }}$ (plot within plot Fig. 2.6 to accommodate for difference in scale). Dextran is not retained in the column, therefore the extra-column volume is quite large in comparison to the retention volume of dextran and the ratio of retention times rather small. Further the peak variance caused by the column is low. Even though the dextran sample is polydisperse in size, it is unlikely that even the smaller fractions enter any of the pore space in a resin with a molecular cut-off just above glucose. Two representative peak overlays, zero length column in Fig. 2.10 and system peaks of $\mathrm{D}_{2} \mathrm{O}$ in Fig. 2.11, $V_{I n j .}=200 \mu \mathrm{L}$, at four velocities were added to the supplementary material.

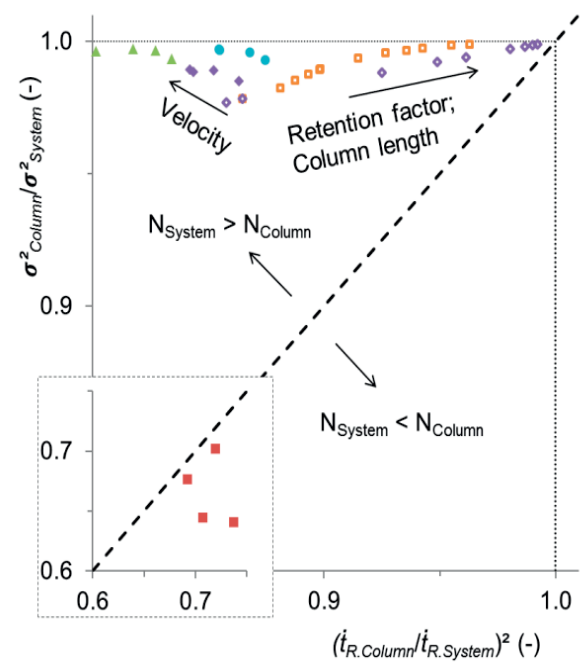

Fig. 2.6 - Ratio of column variance to system variance plotted over the square of the ratio of mean retention times of the column over the system. Experimental data for $\mathrm{D}_{2} \mathrm{O} \bullet$, glucose $\Delta$, urea and dextran $\bullet$. Lumped kinetic model calculation for increased retention factor $k^{\prime} \square$ or column length $L \diamond$. Plot in plot window for dextran, to accommodate for difference in scale.

Relative importance of extra-column contribution can be reduced by increasing the retention time in the column [12,17], e.g. through longer columns or increased retention factors. Therefore, elution profiles of $\mathrm{D}_{2} \mathrm{O}$ were simulated with increased retention factor $k^{\prime}$ and column length $L$. It was found that with increasing $k$ ' and $L$ difference in ratios of mean retention time and variance became smaller, but $N_{\text {System }}$ never became smaller than $N_{\text {Column }}$ (open symbols in Fig. 2.6, increasing $k$ ' and $L$ indicated by top right arrow). The accuracy 
of the simulated peaks was demonstrated in a parity plot of calculated data with fitted parameter $D_{p}$ over measured data for column variance and retention volume (Fig. 2.12, supplementary material).

A relationship of $N_{\text {System }}$ larger than $N_{\text {Column }}$ has, to our knowledge, not been described in literature before. In certain publications $[6,15] N_{\text {Column }}$ is displayed as a function of variance alone. Neglect of extra-column retention time $\left(t_{r \text {.Column }} / t_{\text {.System }}=1\right)$ inevitably leads to a $N_{\text {Column }}$ larger than $N_{\text {System }}$ as can be seen in Fig. 6; when the value on the x-axis is fixed at 1 , only $N_{\text {Column }}$ larger than $N_{\text {System }}$ is mathematically possible.

In the case of $\mathrm{D}_{2} \mathrm{O}$, glucose, and urea, $N_{\text {Column }}$ was overestimated when extra-column volume contribution to band broadening and mean retention time was not taken into account. Therefore the effect of extra-column contribution on scaling up was investigated next.

\subsubsection{System efficiency as function of variance and relative extra-column volume}

Extra-column contribution to retention time and variance has led to a measured system efficiency $\left(N_{\text {System }}\right)$ larger than the deduced column efficiency $N_{\text {Column }}$. The origin of this unexpected behavior was found in ratios of column variance to system variance as well as column retention time to system retention time (chapter 0 ). In case extra-column contribution was not accounted for, $N_{\text {Column }}$ was either under- or overestimated, an error quantified as ratio of $N_{\text {System }}$ to $N_{\text {Column }}$. This error was investigated as a function of extra-column contribution to variance for different extra-column volumes (Fig. 2.7). When extra-column contribution to band broadening and retention was not accounted for in the measured data of retained tracer molecules, the column performance was overestimated. Using system data for a scale-up would overestimate the column efficiency by 15 through $18 \%$ in case of $\mathrm{D}_{2} \mathrm{O}$ and up to $20 \%$ in case of glucose. Again dextran exhibited a behavior of different from retained tracers, as explained in chapter 0.

For Fig. 2.7 increasing retention volume $V_{R \text {.Column }}$ and variance $\sigma^{2}$ Column of a fictional column were used to create different column efficiencies $N_{\text {Column }}$. Extra-column volume $V_{R . E C C}$ and variance $\sigma_{E C C}^{2}$ were used as measured in the laboratory set up, $N_{\text {System }}$ was calculated by summing up retention volumes and variances; analogue to equation 2.1. The influence of retention volume is shown as ratio of $V_{R . E C C}$ to retention volume of a tracer molecule in the system $V_{R . S y s t e m}$, labelled relative extra-column volume $r E C V$.

Experimental data were introduced into Fig. 2.7, as well as data taken from Alexander et al. [14], who investigated extra-column contribution for different tracers and system modifications. All data retrieved from their publication shows $N_{\text {System }}>N_{\text {Column }}$. Retention volumes were calculated based on the retention factors given in their article, the $r E C V$ varied from $1 \%$ through $14 \%$. For the system analyzed in this work $r E C V$ was $6.5 \%$ for $\mathrm{D}_{2} \mathrm{O}, 5.9 \%$ for urea, $7.7 \%$ for glucose, and $12.8 \%$ for dextran.

The column efficiency was lower than the system efficiency when $r E C V>1 \%$ and variance ratios larger than $\sim 50$. With increasing $r E C V$, the threshold of variance ratio at which $N_{\text {System }} / N_{\text {Column }}<1$, became smaller. For variance ratios of about 50 and larger, $N_{\text {System }} / N_{\text {Column }}$ was only influenced by the $r E C V$. The physical background to explain the role of the extra-column volume is found in its different contribution to retention time and variance. Extra-column volume has a relatively great influence on retention time, but adds relatively little to variance, therefore the apparent separation efficiency increases, when the extra-column contribution is accounted for. 


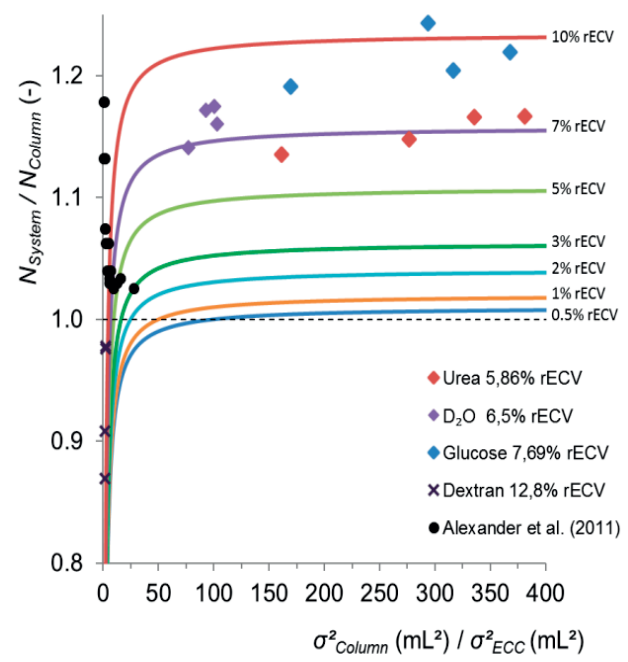

Fig. 2.7 - The error in column efficiency calculation $N_{\text {System }} / N_{\text {Column }}$ made if the influence of the extra-column contribution is not accounted for, plotted for different ratios of column variance to extra-column variance and for different relative extra-column volumes $r E C V$. Additionally added experimental data as well as data taken from Alexander et al. [14].

In most preparative chromatographic applications the ratio of variances from column to extra-column contribution will be quite large, with preparative chromatography employing large bead diameters and high velocities, leading to $N_{\text {System }} / N_{\text {Column }}$ to be determined by $r E C V$ alone. In order to reduce the error made, the ratio of variances $\sigma_{\text {Column }}^{2}$ over $\sigma_{E C C}^{2}$ has to be decreased. This can be obtained by using a more efficient column, for example a column packed with resin particles with a smaller diameter.

In the analytical field resolution is used as a separation criteria. Resolution, as calculated in [33], is dependent on separation factor, capacity factor and column efficiency. The resolution is therefore influenced by the extracolumn contribution to variance as well as retention volume. Due to the dependence on column efficiency, the error made, when not taking the extra-column volume into account, behaves analogue to the ratio $N_{\text {System }}$ over $N_{\text {Column }}$ as seen in Fig. 2.7: actual resolution in the column may be over- or underestimated, depending on the ratio of $\sigma_{\text {Column }}^{2}$ over $\sigma_{E C C}^{2}$ and increases with increasing $r E C V$.

Next to column efficiency, the separation factor $\alpha_{i, j}$ is an important design parameter. The separation factor, calculated as described in [33] is influenced by the extra-column volume through its contribution to mean retention volume $V_{R}$. When the extra-column volume is not taken into account, it will lead to an underestimation of column separation factor and an overestimation of the required chromatographic volume. It could therefore partly compensate the underestimation based on the column efficiency. The measured separation factor $\alpha_{i, j . S y s t e m}$ will be smaller than the actual separation factor in the column $\alpha_{i, j . \text { Column }}$. This 
underestimation is in the range of $1-5 \%$ for $\alpha_{i, j . S y s t e m}$ below 2 and $r E C V=10 \%$ (relative to the retention volume of the first peak).

Influence of correction for extra-column contribution on HETP measured for the system is illustrated in Fig. 2.8, which shows the averaged $H_{E T P}$ System $_{\text {f }}$ for three pulse injections of $200 \mu \mathrm{L} \mathrm{D}_{2} \mathrm{O}$ and the corrected $H_{\text {ETP }}$ Column, as well as the standard deviation. The standard deviation of the column is the sum of standard

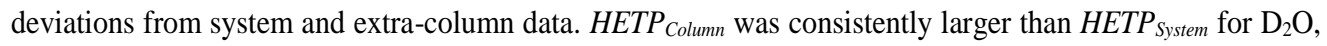
displaying a misconception of the term efficiency loss through extra-column contribution. Through the difference in slope of the van Deemter plot, the overestimation of column efficiency, when not correcting for extra-column contribution, becomes more pronounced with increasing velocity. In case laboratory data are not corrected for extra-column contribution, column efficiency may well be overestimated, leading to underdimensioning in a subsequent scale-up operation.

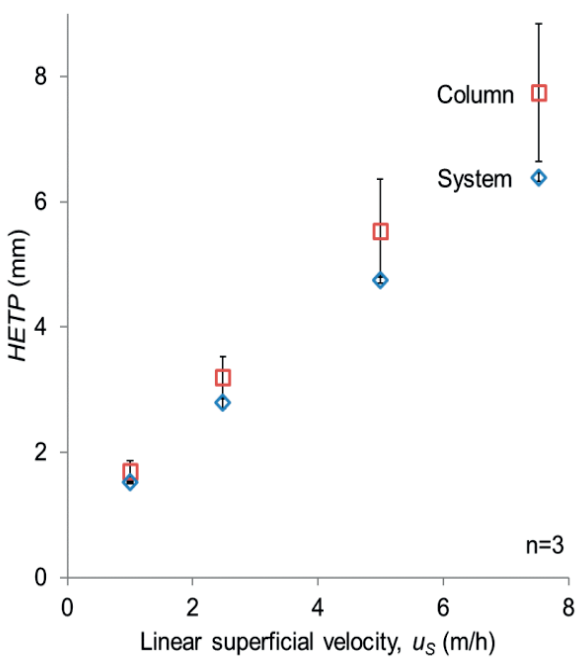

Fig. 2.8 - Mean HETP of triplicate $200 \mu \mathrm{L} \mathrm{D}_{2} \mathrm{O}$ pulse injection as a function of velocity (van Deemter plot) for the system $(\diamond)$ as evaluated from the chromatogram and for the column (a), corrected for extra-column influence as shown in equation 2.1 using data measured with the zero length column. All points are averages of triplicate measurements with standard deviation shown.

\subsection{Conclusions}

Accurate estimation of column performance can only be accomplished if the contribution of extra-column volume to variance $\sigma_{E C C}^{2}$ and mean retention time $\bar{t}_{R . E C C}^{2}$ are accounted for. Measuring the extra-column contribution with a $Z D V$ consistently underestimates $\sigma_{E C C}^{2}$ and $\bar{t}_{R E C C C}^{2}$. Although differences are small, on 
average 3\% difference in efficiency measured with $Z D V$ to the zero length column, it is advised to use a zero length column when extra-column contribution needs to be determined with accuracy.

Extra-column contribution and column performance should be analyzed under the same conditions [7], but we show injection volume, volumetric flow rate, and tracer molecule type can be accounted for with reasonable accuracy, as long as the molecules have a similar diffusive behavior. With this knowledge, for a given experimental set-up, extra-column contribution does not require to be measured for every tracer, injection volume, or velocity. Contradicting literature we show that influence of injection volume on measured variance is not constant but a function of velocity.

The term efficiency loss may lead to the impression, that neglecting extra-column contribution to overall band broadening in a small scale system, will lead to a safety margin in column dimensions when scaling up the process. This is however a misconception of the term efficiency loss, since calculated system efficiency may well be higher than column efficiency. In neglecting extra-column contribution, column efficiency may be overestimated by as much as $20 \%$. This effect is especially pronounced for columns with low inherent efficiency, as are often utilized in preparative separations. To avoid confusion about efficiencies of systems and columns we suggest that the terms efficiency and HETP are only used for column data, after accounting for extra-column contribution.

\subsection{Acknowledgements}

This research took place within the framework of the Institute for Sustainable Process Technology ISPT. The authors would like to thank the ISPT for their support, together with Unilever (Vlaardingen, NL), FrieslandCampina Research (Amersfoort, NL), DSM (Delft, NL) and Cosun Food Technology (Roosendaal, $\mathrm{NL}$ ) for their financial support and interest in this project.

\subsection{References}

1. J.C. Sternberg, Extracolumn contributions to chromatographic band broadening, Advances in Chromatography, 2 (1966) 205-270.

2. J.J. Kirkland, W.W. Yau, H.J. Stoklosa, C.H. Dilks, Sampling and Extra-Column Effects in HighPerformance Liquid Chromatography; Influence of Peak Skew on Plate Count Calculations, J.Chromatogr.Sci., 15 (1977) 303-316.

3. G. Guiochon, D.G. Shirazi, A. Felinger, A.M. Katti, Fundamentals of Preparative and Nonlinear Chromatography, Academic Press, 2006.

4. M. Martin, C. Eon, G. Guiochon, Study of the pertinency of pressure in liquid chromatography, Journal of Chromatography A, 108 (1975) 229-241.

5. A. Prüß, C. Kempter, J. Gysler, T. Jira, Extracolumn band broadening in capillary liquid chromatography, Journal of Chromatography A, 1016 (2003) 129-141.

6. K.J. Fountain, U.D. Neue, E.S. Grumbach, D.M. Diehl, Effects of extra-column band spreading, liquid chromatography system operating pressure, and column temperature on the performance of sub-2- $\mu$ m porous particles, Journal of Chromatography A, 1216 (2009) 5979-5988. 
7. D.V. McCalley, Instrumental considerations for the effective operation of short, highly efficient fused-core columns. Investigation of performance at high flow rates and elevated temperatures, Journal of Chromatography A, 1217 (2010) 4561-4567.

8. F. Gritti, G. Guiochon, Effect of the pressure on pre-column sample dispersion theory, experiments, and practical consequences, Journal of Chromatography A, 1352 (2014) 20-28.

9. J.P. Grinias, B. Bunner, M. Gilar, J.W. Jorgenson, Measurement and Modeling of Extra-Column Effects Due to Injection and Connections in Capillary Liquid Chromatography, Chromatography, 2 (2015) 669.

10. R. De Pauw, K. Shoykhet, G. Desmet, K. Broeckhoven, Understanding and diminishing the extracolumn band broadening effects in supercritical fluid chromatography, Journal of Chromatography A, 1403 (2015) 132-137.

11. K. Vanderlinden, K. Broeckhoven, Y. Vanderheyden, G. Desmet, Effect of pre- and post-column band broadening on the performance of high-speed chromatography columns under isocratic and gradient conditions, Journal of Chromatography A, 1442 (2016) 73-82.

12. G. Guiochon, The limits of the separation power of unidimensional column liquid chromatography, Journal of Chromatography A, 1126 (2006) 6-49.

13. A. Jupke, A. Epping, H. Schmidt-Traub, Optimal design of batch and simulated moving bed chromatographic separation processes, 14th International Symposium on Preparative and Process Chromatography, 944 (2002) 93-117.

14. A.J. Alexander, T.J. Waeghe, K.W. Himes, F.P. Tomasella, T.F. Hooker, Modifying conventional high-performance liquid chromatography systems to achieve fast separations with Fused-Core columns: A case study, Journal of Chromatography A, 1218 (2011) 5456-5469.

15. N. Wu, A.C. Bradley, Effect of column dimension on observed column efficiency in very high pressure liquid chromatography, High Speed and High Resolution Separations. In Honour of Professor Milton Lee on the Occasion of his 65th Birthday, 1261 (2012) 113-120.

16. A.C. Sanchez, J.A. Anspach, T. Farkas, Performance optimizing injection sequence for minimizing injection band broadening contributions in high efficiency liquid chromatographic separations, HighPerformance Columns and their Operations: The Speed, Efficiency and Peak Capacity Obtainable with High-Performance Columns under Various Operation Conditions, 1228 (2012) 338-348.

17. F. Gritti, G. Guiochon, Accurate measurements of peak variances: Importance of this accuracy in the determination of the true corrected plate heights of chromatographic columns, Journal of Chromatography A, 1218 (2011) 4452-4461.

18. F. Gritti, G. Guiochon, Mass transfer kinetics, band broadening and column efficiency, Journal of Chromatography A, 1221 (2012) 2-40.

19. G. Carta, A. Jungbauer, Effects of Dispersion and Adsorption Kinetics on Column Performance, Protein Chromatography, Wiley-VCH Verlag GmbH \& Co. KGaA, 2010, pp. 237-276.

20. J.J. Baeza-Baeza, S. Pous-Torres, J.R. Torres-Lapasió, M.C. García-Álvarez-Coque, Approaches to characterise chromatographic column performance based on global parameters accounting for peak broadening and skewness, Journal of Chromatography A, 1217 (2010) 2147-2157.

21. F. Gritti, A. Felinger, G. Guiochon, Influence of the errors made in the measurement of the extracolumn volume on the accuracies of estimates of the column efficiency and the mass transfer kinetics parameters, Journal of Chromatography A, 1136 (2006) 57-72. 
22. H.H. Lauer, G.P. Rozing, The selection of optimum conditions in HPLC I. The determination of external band spreading in LC instruments, Chromatographia, 14 (1981) 641-647.

23. F. Gritti, G. Guiochon, Accurate measurements of the true column efficiency and of the instrument band broadening contributions in the presence of a chromatographic column, Journal of Chromatography A, 1327 (2014) 49-56.

24. A. Micke, M. Kočiřik, M. Bülow, Theory of zero length column chromatography with the condition of a well-stirred sorbing zone, Microporous Materials, 1 (1993) 373-381.

25. R.P.W. Scott, Extra-column dispersion in liquid chromatography systems, J.Liq.Chromatogr.Rel.Technol., 25 (2002) 2567-2587.

26. J. Samuelsson, L. Edström, P. Forssén, T. Fornstedt, Injection profiles in liquid chromatography. I. A fundamental investigation, Journal of Chromatography A, 1217 (2010) 4306-4312.

27. P. Aggarwal, K. Liu, S. Sharma, J.S. Lawson, H. Dennis Tolley, M.L. Lee, Flow rate dependent extracolumn variance from injection in capillary liquid chromatography, Journal of Chromatography A, 1380 (2015) 38-44.

28. D. Eisenberg, Kauzmann, W., The structure and properties of water, J.Mol.Struct., 6 (1970) 419.

29. P. Gribbon, T.E. Hardingham, Macromolecular diffusion of biological polymers measured by confocal fluorescence recovery after photobleaching, Biophys.J., 75 (1998) 1032-1039.

30. A.C.F. Ribeiro, O. Ortona, S.M.N. Simões, C.I.I.A.V. Santos, P.M.R.A. Prazeres, A.J.M. Valente, V.M.M. Lobo, H.D. Burrows, Binary Mutual Diffusion Coefficients of Aqueous Solutions of Sucrose, Lactose, Glucose, and Fructose in the Temperature Range from (298.15 to 328.15) K, Journal of Chemical \& Engineering Data, 51 (2006) 1836-1840.

31. A. Tamir, O. Falk, Dissolution of solids and pressure drop in a cyclone semibatch reactor and a two impinging streams semibatch reactor, Ind Eng Chem Res, 27 (1988) 1930-1936.

32. A. Felinger, G. Guiochon, Comparison of the Kinetic Models of Linear Chromatography, Chromatographia, 60 (2004) S175-S180.

33. A. Seidel-Morgenstern, M. Schulte, A. Epping, H. Schmidt-Traub, M. Schulte, A. SeidelMorgenstern, Fundamentals and General Terminology, Preparative Chromatography, Wiley-VCH Verlag GmbH \& Co. KGaA, 2012, pp. 7-46. 


\subsection{Supplementary material}

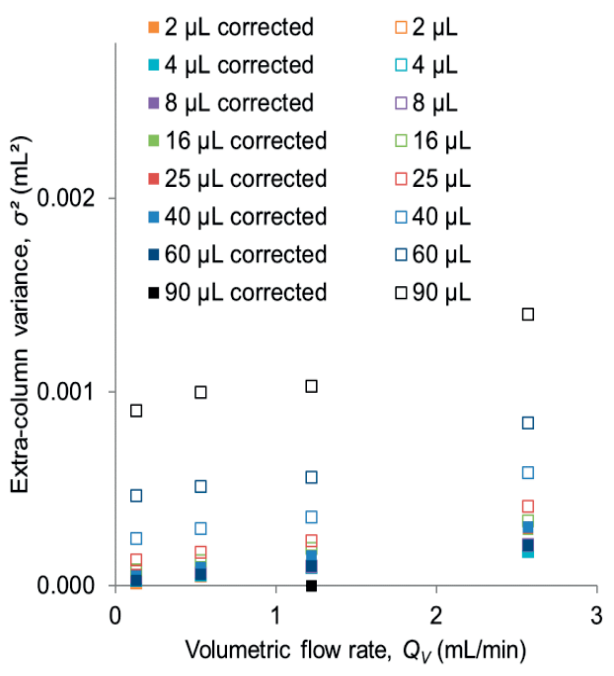

Fig. 2.9 - Variance of extra-column volume, corrected for injection volume with $D^{2}=\mathrm{f}\left(\mathrm{Q}_{\mathrm{v}}\right)$ as shown in Chapter 4.2. Data from Lauer \& Rozing [22].

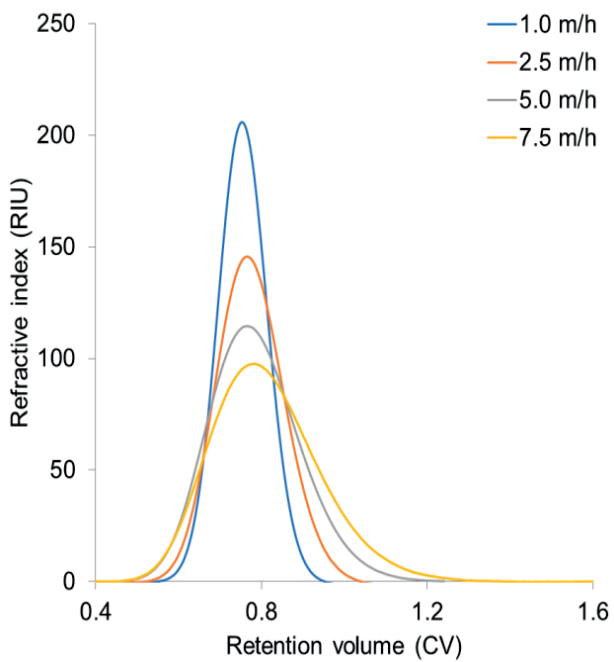

Fig. 2.11 - Pulse injection profiles of $\mathrm{D}_{2} \mathrm{O}$ after passing through the system (column and extra-column volume) at four velocities. $V_{I n j}=200 \mu \mathrm{L}$.

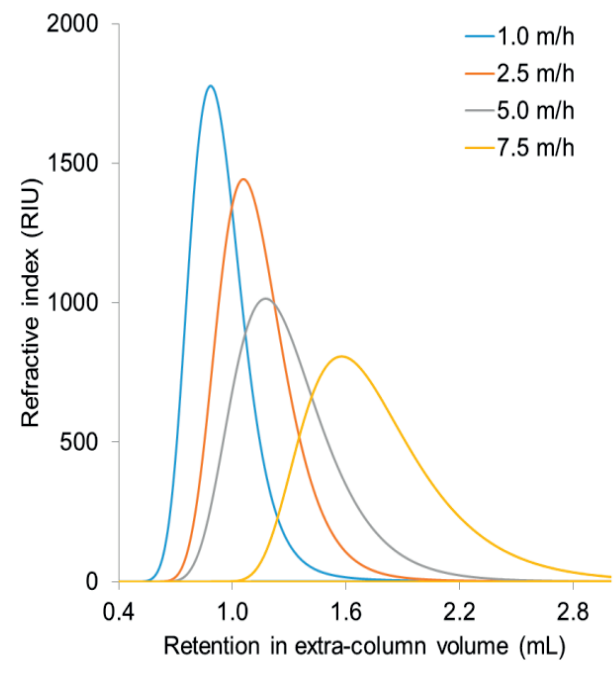

Fig. 2.10 -Pulse injection profiles of $\mathrm{D}_{2} \mathrm{O}$ after passing through extra-column volume with zero length column at four velocities. $V_{I n j .}=200 \mu \mathrm{L}$.

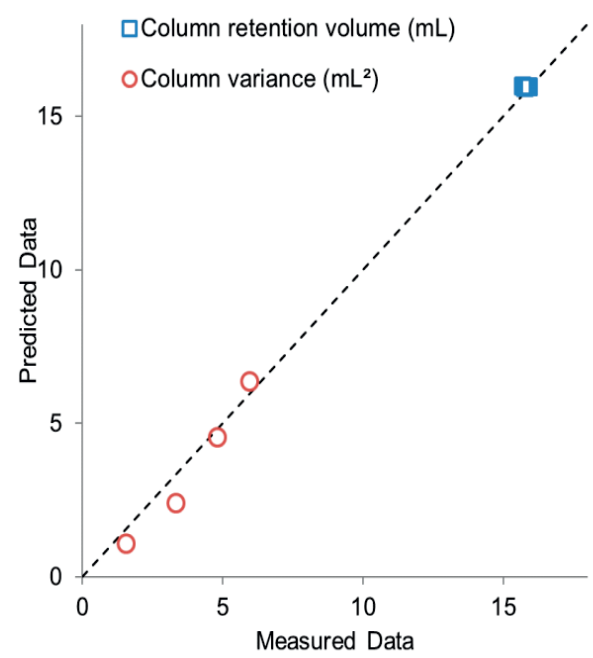

Fig. 2.12 - Parity plot of fitted data over measured data for column variance and retention volume to show accuracy of model calculations. 



\section{Chapter 3}

\section{Predicting intraparticle diffusivity as function of stationary phase characteristics in preparative chromatograph}

This chapter has been submitted as:

A. Schultze-Jena, M.A. Boon, D.A.M. de Winter, P.J.Th. Bussmann, A.E.M. Janssen, A. van der Padt. 


\section{Abstract}

Diffusion inside pores is the rate limiting step in many preparative chromatographic separations and a key parameter for process design in weak interaction aqueous chromatographic separations employed in food and bio processing. This work aims at relating diffusion inside porous networks to properties of stationary phase and of diffusing molecules. Intraparticle diffusivities were determined for eight small molecules in nine different stationary phases made from three different backbone materials. Measured intraparticle diffusivities were compared to the predictive capability of the correlation by Mackie and Meares and the parallel pore model. All stationary phases were analyzed for their porosity, apparent pore size distribution and tortuosity, which are input parameters for the models. The parallel pore model provides understanding of the occurring phenomena, but the input parameters were difficult to determine experimentally. The model predictions of intraparticle diffusion were of limited accuracy. We show that prediction can be improved when combining the model of Mackie and Meares with the fraction of accessible pore volume. The accessible pore volume fraction can be determined from inverse size exclusion chromatographic measurements. Future work on our improved model should be further challenged, specifically widening the applicability to greater accessible pore fractions $(>0.7)$ with corresponding higher intraparticle diffusivities $\left(D_{p} / D_{m}>0.2\right)$. A database of intraparticle diffusion and stationary phase pore property measurements is supplied, to contribute to general understanding of the relationship between intraparticle diffusion and pore properties. 


\subsection{Introduction}

Diffusion inside porous structures is of relevance in fields like genomics, biofilms, drug delivery, implantable devices, contact lenses, cell- and tissue engineering, geography, petroleum recovery, heterogeneous catalysis, membrane filtration and chromatography [1-13]. Well over a hundred years of research has resulted in a wide range of definitions and quantifications of pore characteristics and diffusivity correlations, even within single scientific disciplines [14, 15]. In chromatographic processes, porous structures are essential for separation as pores increase surface area for adsorption and/or reaction processes and separate molecules by structural properties. Chromatographic separation is achieved by pumping feed (mobile phase) through a bed of microporous stationary phase. In packed bed chromatography the stationary phase consists of spherical particles or in some cases a single solid monolithic structure. Mass transfer, from the mobile phase into the stationary phase and back is limited by the rate in which molecules enter, exit, and move through the stationary phase. The molecular movement is particularly important when relatively large distances have to be traversed by diffusive forces [16-18]. This is often the case in preparative chromatography, where large particle diameters are desired for large volumetric feed throughput while maintaining low back pressures. The limitation of mass transfer through intraparticle diffusivity becomes even more relevant with increasing mobile phase velocity [19]. Effectively, resistance to intraparticle diffusion increases separation time [17] and thus reduces productivity. Understanding mass transfer inside the stationary phase is an important step towards optimizing chromatographic processes. Unfortunately, accurately predicting intraparticle diffusion remains challenging $[17,18]$.

The term intraparticle diffusivity describes the diffusion inside a porous particle and should not be confused with interparticle diffusivity, which describes diffusion between different particles. Methods to describe intraparticle diffusivity in detail are as diverse as the fields themselves, since particular challenges, scales, and technological limitations vary in each field. In membrane ultrafiltration for instance, pore geometry is often assumed to resemble straight cylindrical tubes with the same length as the membrane thickness [20]. Such an assumption is not valid in chromatography. The only similarity of the existing theories and models is the dependence of intraparticle diffusivity on free- or self-diffusion in bulk medium, usually described in terms of Fick diffusion. Intraparticle diffusivity is thus described as bulk diffusivity, limited through one or more constraints both inherent to pore properties as well as interplay with properties of diffusing molecules. Hence the term effective diffusivity is often used.

The reduced diffusion in porous matrices and gels is described by a number of models, both empirical and analytical. A very comprehensive model is the parallel pore model, which describes the reduction of intraparticle diffusivity through particle porosity, sterical hindrance and obstruction to diffusion [21]. Within gels, diffusion is often described on the basis of gel volume fraction and the ratio of polymer strain radius to target molecule size [22]. The identification and quantification of all parameters affecting diffusivity inside stationary phases is challenging, largely due to the interplay between different parameters. Furthermore, the definitions of these parameters leave room for different interpretations and their quantification often involves indirect measurements, approximations, and/or fitting.

Our work aims at gaining further insight into individual contributions of pore characteristics and their respective relation to intraparticle diffusivity. Intraparticle diffusivity was measured in size exclusion mode via van Deemter curves and compared to stationary phase properties. Stationary phases were analyzed for 
their porosity, apparent pore size distribution, and particle tortuosity. Electron microscopy was attempted to independently confirm pore characteristics. Intraparticle diffusivities of eight different small molecules were measured in chromatographic stationary phases of three different backbone materials. For each backbone material three different stationary phases of the same series, but with a different degree of cross-linking, were analyzed. The data was used to compare the predictive capabilities of the Mackie and Meares correlation and the parallel pore model.

\subsection{Theoretical background}

\subsubsection{Diffusion}

Diffusion is the stochastic motion of molecules. Without any constraints, the diffusive motion is called free-, self- or bulk diffusion. The net ensemble movement due to a spatial difference in concentrations can be described with Maxwell-Stefan or Fick-equations. In a thermodynamically ideal system, the diffusion coefficients of Fick and Maxwell-Stefan are identical [23]. As diffusion inside chromatographic particles is often considered to happen in dilute and ideal systems, Fick diffusion coefficients are used to describe and quantify diffusive mass transfer in chromatography. In case of diffusion within a porous medium with pore dimensions in the order of magnitude of the molecular free path, diffusivity is effectively reduced. Intraparticle diffusivity can thus be described as bulk diffusivity, limited through one or more constraints inherent to pore characteristics. Hence terms such as 'apparent-' or 'effective diffusivity' are often used. In case of pores smaller than the molecular free path, diffusion is sometimes called 'Knudsen diffusion'. Different diffusion rates for the same molecules in a different porous structures can be explained by acknowledging that different pore structures reduce bulk diffusivity differently. In addition to that, molecules adsorbed on pore surfaces may diffuse as well, which is described as 'surface diffusion' [24, 25]. In all cases discussed here, molecular transport within the porous structures is considered to be purely diffusion driven without any contribution of convection.

Overall resistance to mass transfer inside a chromatographic column is the combined result of longitudinal diffusion along the column, eddy dispersion, external film mass transfer resistance, mass transfer resistance inside the pores of the stationary phase, rate of adsorption and desorption as well as the friction-expansion of the mobile phase [26]. As a result, a pulse injected into the column results in a broadened peak in the eluate. Measuring the eluate concentration in time allows for the construction of a so-called van Deemter curve by measuring mean retention time and peak variance eluted at different linear velocities. In preparative chromatography, which generally operates at high velocities using large stationary phase particles, the overall mass transfer is generally limited by resistance to diffusion inside the porous region of the stationary phase [27]. The extend of this limitation is such, that in the linear region of a van Deemter curve, measured under preparative conditions, the slope is almost entirely dependent on intraparticle mass transfer resistance, which in turn can be derived from the slope of the curve, while accounting for the contribution of film mass transfer resistance [27]. 


\subsubsection{Predictive models}

In literature a range of both empirical and theoretical models can be found describing diffusion inside porous matrices. Generally, diffusion is always described as Fickian diffusion. In the models the ratio of intraparticle diffusivity $D_{p}$ over bulk diffusivity $D_{m}$ is set in relation to one or more terms describing the stationary phase or an interaction between stationary phase and diffusing molecule. The majority of predictive models use the particle porosity $\varepsilon_{p}$ to correlate intraparticle diffusion to a property of the stationary phase which yields the intuitive boundaries $\lim _{\varepsilon_{P} \rightarrow 0} D p / D m=0$ and $\lim _{\varepsilon_{P} \rightarrow 1} D p / D m=1$. Overviews of different proposed empirical, semi-empirical, and theoretical expressions relating $\varepsilon_{p}$ to intraparticle diffusion are given in [14, $28]$.

\section{Correlation by Mackie and Meares}

In chromatography the correlation of Mackie and Meares (equation 3.1), as described by Guiochon [18], is often used. While the intuitive boundary conditions of diffusion in porous space are met, the model of Mackie and Meares, developed for electrolyte diffusion in ion-exchange membranes, takes neither characteristics of diffusing molecules nor structures and dimensions of pores into account. Yet, due to its simplicity and measurability of the single parameter particle porosity $\varepsilon_{p}$, this model offers an attractive method for a first estimation of $D_{p} / D_{m}$.

$$
D_{P}=\left[\frac{\varepsilon_{P}}{2-\varepsilon_{P}}\right]^{2} D_{m}
$$

\section{The parallel pore model}

The probably most commonly used model to relate intraparticle diffusivity to pore and molecule characteristics is the parallel pore model (equation 3.2) [29, 30]. The model is based on the assumption that diffusivity inside a porous network is comparable to diffusion inside straight parallel cylindrical tubes, where diffusion can only take place inside the pores and not through the solid phase of the pore walls [21].

For non-adsorptive processes, the parallel pore model describes an intraparticle diffusion $D_{p}$, as bulk diffusion $D_{m}$ reduced by the characteristics of the solid phase: the porosity $\varepsilon_{p}$, hindrance diffusion factor $F\left(\lambda_{m}\right)$, and the internal obstruction factor $\gamma_{p}$, all three of which have values between zero and one.

$$
D_{p}=\varepsilon_{p} \cdot F\left(\lambda_{m}\right) \cdot \gamma_{p} \cdot D_{m}
$$

A term describing surface diffusion is added to the parallel pore model in adsorptive processes [24, 25]. In reversed phase liquid chromatography applications, surface diffusion may become the major contributor to intraparticle diffusion [31].

\subsubsection{Particle porosity $\varepsilon_{p}$}

Particle porosity $\varepsilon_{p}$ refers to the pore volume accessible to the mobile phase, inside the particles. It is important to realize the influence of different measurement methods for particle porosity. Generally, particle porosity 
should be measured under the same conditions as chromatographic measurement, as particle porosity is not necessarily an intrinsic particle property. Particles may be subject to swelling and/or shrinking with medium composition and temperature [16]. During adsorptive processes, particle porosity may be influenced through adsorbed molecules, which block otherwise accessible pore volume [32].

Particle porosity can be measured ex- or in situ. Two methods to measure particle porosity ex situ are electron microscopy and intrusion porosimetry with nitrogen or mercury [16] [33]. Both methods require measurements in vacuum, which potentially leads to deformation of many chromatographic stationary phases. Hence caution is required when interpreting the results [13]. In situ measurement of particle porosity $\varepsilon_{p}$ in chromatographic stationary phases usually encompasses elution volume measurements of two non-retained molecules of different size: one small molecule capable of accessing the entire particle pore volume and the other a large molecule incapable of entering the particle pore volume at all. The former measures the total porosity $\varepsilon_{t}$, the latter the interparticle-, bed-, or external porosity $\varepsilon_{e}$. From these two measurements, the particle porosity is calculated with equation 3.3[18]:

$$
\varepsilon_{p}=\frac{\varepsilon_{t}-\varepsilon_{e}}{1-\varepsilon_{e}}
$$

\subsubsection{Hindrance diffusion factor $F\left(\lambda_{m}\right)$}

The second term in equation 3.2, the hindrance diffusion factor $F\left(\lambda_{m}\right)$, describes the drag a diffusing molecule experiences due to confinement within pore walls as well as steric exclusion [34]. For molecules larger than roughly $1 / 10^{\text {th }}$ of pore diameter, mobility will be markedly reduced through friction with pore walls [35]. Different relationships can be found in literature to describe this phenomenon, mostly based on the ratio of molecule to pore radii $\lambda_{m}$ and the work of Renkin [36] and Brenner and Gaydos [25]. Dechadilok and Deen [20] improved an empirical expression which had been developed through many researchers over the years and which now fits the range of $0 \leq \lambda_{m} \leq 0.95$ (equation 3.4). Equation 3.4was developed to describe hindered diffusion in pores of membranes in absence of convection, assuming pores to be straight and cylindrical. The width of pore size distribution is not taken into account as $\lambda_{m}$ is calculated from the mean pore radius.

$$
\begin{aligned}
F\left(\lambda_{m}\right)=1+\frac{9}{8} & \lambda_{m} \ln \lambda_{m}-1.56034 \lambda_{m}+0.528155 \lambda_{m}{ }^{2}+1.91521 \lambda_{m}{ }^{3} \\
& -2.81903 \lambda_{m}{ }^{4}+0.270788 \lambda_{m}{ }^{5}+1.10115 \lambda_{m}{ }^{6}-0.435933 \lambda_{m}{ }^{7}
\end{aligned}
$$

\subsubsection{Internal obstruction factor $\gamma_{p}$}

The internal obstruction factor $\gamma_{p}$ is arguably the most ambiguous contribution to the parallel pore model. The ambiguity in literature originates from different concepts for contributing mechanisms to $\gamma_{p}$, which are often difficult to validate experimentally [28, 37-39]. Giddings suggested that the internal obstruction factor $\gamma_{p}$ is the product of obstruction due to constriction $\gamma_{p \text {,cons }}$ and obstruction due to tortuosity $\gamma_{p . \tau}$ [40]. In more recent definitions the obstruction due to mesopore (2-50 nm [41]) connectivity $\gamma_{p, c o n n}$ is attributed to $\gamma_{p}$ as well [6], leading to equation 3.5: 


$$
\gamma_{p}=\gamma_{p, \text { cons }} \cdot \gamma_{p, \tau} \cdot \gamma_{p, \text { conn }}
$$

In practice $\gamma_{p}$ may be difficult to distinguish from $F\left(\lambda_{m}\right)[42,43]$. For this reason $\gamma_{p}$ is often used as a fitting parameter which then sums up all contributions that obstruct diffusion within the pore volume, as well as any experimental errors. While this works for retrofitting a model to a particular system, little contribution is made to fundamental understanding of the relationship of intraparticle diffusion and pore structures. Nevertheless, it is useful to discuss the three different internal obstruction factors, as it exemplifies the complexity of diffusive molecular transport through a porous material.

\section{Obstruction due to constriction}

Constriction describes randomly located bottlenecks in diffusion paths inside the porous matrix, which slow down molecules [37, 44]. Wiedenmann et al. [45] calculate the constriction factor $\gamma_{p, c o n s}$ with equation 3.6 from data obtained from three dimensional images of pore structures via x-ray tomography.

$$
\gamma_{p, \text { cons }}=\frac{A_{\min }}{A_{\max }}=\frac{\pi r_{\min }^{2}}{\pi r_{\max }^{2}}
$$

In order for equation 3.6 to be of any practical use, the transport relevant radii, $r_{\min }$ the smallest and $r_{\max }$ the largest pore radius a diffusing molecule encounters in a porous matrix, must be determined. This however, is not possible without detailed information on three dimensional pore structure, which presents a technical challenge for microscopy techniques beyond the scope of this paper. Due to the complexity and interdependence of all factors contributing to $\gamma_{p}$, the actual value of $\gamma_{p, \text { cons }}$ cannot be validated in practice [45].

\section{Obstruction due to tortuosity}

Obstruction to diffusion due to tortuosity $\gamma_{p, \tau}$ of porous particles is assumed to be a constant of the porous network and independent of molecular species, according to theories proposed by Giddings [40]. The obstruction to diffusion due to tortuosity $\gamma_{p, \tau}$ was calculated from measured tortuosity $\tau_{p}$ via equation 3.7 :

$$
\gamma_{p, \tau}=\frac{1}{\tau_{p}^{2}}
$$

Tortuosity $\tau_{p}$ is defined as ratio of average pore length $L_{p}$ to length of the porous medium or particle diameter $d_{p}$ and since $L_{p}>d_{p}$, it follows that $\tau_{p}>1$ [39]. This definition makes tortuosity difficult to determine, as it is not reducible to classic measurable microscopic parameters [46]. Tortuosity can be measured via electric impedance, either inside the column [47] or from column packing material in suspension [46] and generally increases with decreasing porosity [21]. Extensive discussions on tortuosity can be found in literature, e.g. [15, 38, 39, 46, 48-56]. Tortuosities between 1 and 5 [21, 37] are found.

\section{Obstruction due to connectivity}

Pore interconnectivity describes the extent of communication between pores in the 3D space [57]. It is well defined in pore network models, where a number of connections is attributed to each node [58]. A definition for connectivity in situ yields a term, which is hard to quantify: "connectivity describes the average number of possible distinct paths for the molecules of a fluid impregnating the porous material to move from one site 
of this material to another one" [37]. The contribution of connectivity to $\gamma_{p}$ is dependent on the size of the diffusing molecule [59]. Obstruction due to connectivity $\gamma_{p, c o n n}$ is primarily important to small molecules. Larger molecules get increasingly hindered through proximity to pore walls and $F\left(\lambda_{m}\right)$ dominates. Pore network modelling has shown that connectivity can have a large effect on $\gamma_{p}$ [43]. It is unclear however, how connectivity can be measured in situ and how its effect can be isolated from other contributions to $\gamma_{p}$.

\subsection{Materials and methods}

\subsubsection{Materials}

Mobile phase

All experiments were conducted with a phosphate based mobile phase $\left(25 \mathrm{mM} \mathrm{Na}_{2} \mathrm{HPO}_{4}, 25 \mathrm{mM} \mathrm{NaH}_{2} \mathrm{PO}_{4}\right.$, and $50 \mathrm{mM} \mathrm{NaCl}$; all from Merck, Germany) in Milli-Q water. Viscosity was measured with a Physica MCR 301 rheometer (Anton Paar, Austria). Before use the mobile phase was filtered through a $0.45 \mu \mathrm{m}$ Durapore ${ }^{\circledR}$ membrane filter (Merck, Germany).

\section{Stationary phases}

Stationary phases of three different backbone materials (dextran, styrene-divinylbenzene, and hydroxylated methacrylic polymer) were selected. For each backbone material three stationary phases of the same series and a different degree of cross-linking were selected (Table 3.1).

Table 3.1 - Stationary phase series and backbone material of all stationary phases

\begin{tabular}{|c|c|c|}
\hline Stationary phase & Material & Manufacturer \\
\hline Sephadex $G-10$ & \multirow{3}{*}{$\begin{array}{l}\text { Cross-linked } \\
\text { dextran }\end{array}$} & \multirow{3}{*}{ GE Healthcare } \\
\hline Sephadex $G-15$ & & \\
\hline Sephadex G-25 & & \\
\hline Dowex 50WX8 & \multirow{3}{*}{$\begin{array}{l}\text { Styrene- } \\
\text { divinylbenzene }\end{array}$} & \multirow{3}{*}{ Dow Chemical } \\
\hline Dowex 50WX4 & & \\
\hline Dowex 50WX2 & & \\
\hline Toyopearl HW-40F & Hydroxylated & \multirow{3}{*}{ Tosoh Bioscience } \\
\hline Toyopearl HW-50F & methacrylic & \\
\hline Toyopearl HW-65F & polymer & \\
\hline
\end{tabular}

The number in the name of each stationary phase denotes the degree of cross-linking or concentration of crosslinking agent. While the Sephadex and Toyopearl stationary phases are actual size exclusion SEC stationary phases, the Dowex stationary phases are cation exchange stationary phases, that were used in SEC mode. Before final packing, the $\mathrm{H}^{+}$ion of the Dowex stationary phases was exchanged for $\mathrm{Na}^{+}$with $1 \mathrm{M} \mathrm{NaCl}$. Due to the relatively high salt concentration in the mobile phase, no ionic interaction between target molecules and Dowex stationary phases were observed. Particle size distributions were measured with a Mastersizer 2000 (Malvern, UK) in phosphate buffer at room temperature. The Sauter diameter, or surface weighted mean diameter $d_{3,2}$, and its standard deviation was calculated from ten consecutive particle size distribution measurements. 


\section{Target molecules}

Acetone was added per volume into mobile phase and heavy water $\mathrm{D}_{2} \mathrm{O}$ was used undiluted. All solid target molecules were dissolved in the mobile phase. Their respective concentrations, molecular weights, molecule radii and detection wavelengths (refractive index in case of dextran) are listed in Table 3.2. Molecular radii $r_{m}$ were calculated from two equations. For small molecules, up to and including the disaccharide sucrose a spherical shape was assumed and the Stokes radius calculated from Stokes-Einstein relation. For all molecules larger than sucrose, the viscosity radius $R_{h}$ was calculated from the empirical relation to molecular weight $M_{w}$ given in equation 3.8 [60].

$$
R_{h}=0.271 M_{w}^{0.498}
$$

In addition a series of analytical dextran standards Dextran $1 k$ through Dextran $400 k$ was used for pore size distribution measurements. $\mathrm{NaCl}$ was obtained from Merck, Germany, all other molecules from Sigma Aldrich, St. Louis, MO, USA.

Table 3.2 - Target molecules, respective concentration in sample volume, molecular weight, molecular radii and detection wavelength ( $R I$ for refractive index).

\begin{tabular}{|c|c|c|c|c|}
\hline Molecule & $c(g / L)$ & Molecular weight $(\mathrm{Da})$ & Molecule radius (nm) & Detection \\
\hline $\mathrm{D}_{2} \mathrm{O}$ & Pure & 20 & $0.09^{\mathrm{s}}$ & RI \\
\hline$\gamma$-aminobutyric acid & 10 & 103 & $0.26^{\mathrm{s}}$ & $210 \mathrm{~nm}$ \\
\hline Triglycerin & 5 & 189 & $0.34^{\mathrm{s}}$ & $218 \mathrm{~nm}$ \\
\hline Fructose & 10 & 180 & $0.32^{\mathrm{s}}$ & RI \\
\hline Sucrose & 10 & 342 & $0.48^{\mathrm{s}}$ & RI \\
\hline Maltotriose & 10 & 504 & $0.60^{\mathrm{v}}$ & RI \\
\hline Dextran $2 \cdot 10^{6}$ & 10 & $2 \cdot 10^{6}$ & $36.71^{\mathrm{v}}$ & RI \\
\hline $\mathrm{NaCl}$ & 58 & 58 & $0.13^{\mathrm{s}}$ & $200 \mathrm{~nm}$ \\
\hline Acetone & $2 \%(\mathrm{v} / \mathrm{v})$ & 58 & $0.19^{\mathrm{s}}$ & $260 \mathrm{~nm}$ \\
\hline Dextran $1 k$ & 5 & 1,100 & $0.89^{\mathrm{v}}$ & RI \\
\hline Dextran $4 k$ & 5 & 4,400 & $1.77^{\mathrm{v}}$ & RI \\
\hline Dextran $10 k$ & 5 & 10,000 & $2.66^{\mathrm{v}}$ & RI \\
\hline Dextran $20 k$ & 5 & 20,000 & $3.76^{\mathrm{v}}$ & RI \\
\hline Dextran $45 k$ & 5 & 45,000 & $5.63^{\mathrm{v}}$ & RI \\
\hline Dextran $65 k$ & 5 & 65,000 & $6.76^{\mathrm{v}}$ & RI \\
\hline Dextran $125 k$ & 5 & 125,000 & $9.36^{\mathrm{v}}$ & RI \\
\hline Dextran $195 k$ & 5 & 195,000 & $11.68^{\mathrm{v}}$ & RI \\
\hline Dextran $275 k$ & 5 & 275,000 & $13.86^{\mathrm{V}}$ & RI \\
\hline Dextran $400 k$ & 5 & 400,000 & $16.70^{\mathrm{v}}$ & RI \\
\hline
\end{tabular}

\section{Chromatographic equipment}

For liquid chromatography a Wellchrom set-up with a K-1001 pump and a K-2401 RI-detector was used, all from Knauer, Germany. Further a Julabo F25 MP controlled the temperature in the column jacket and a mini Cori-Flow flowmeter (Bronkhorst, The Netherlands) measured the flow rate after the detector. Pressure drop over the column bed was measured using EZG10 pressure sensors (Knauer, Germany), injection port, valves, column, pressure sensors and detectors were connected with 0.02” PEEK tubing (Grace, Deerfield, IL, USA). 
All elution peaks were measured on slurry packed Götec Superformance 300-10 columns (300 x $10 \mathrm{~mm}$ ) with tefzel capillaries of $35 \mathrm{~cm}$ lengths and an inner diameter of $0.5 \mathrm{~mm}$, including flow adapter with frits and filter (all Götec, Germany). Bed height varied with pressure between 29 and $21 \mathrm{~cm}$, the precise bed heights of each stationary phase are listed in the supplementary material in Table 3.5. The zero length column was a Götec Superformance $10-10$ column $(10 \times 10 \mathrm{~mm})$ without stationary phase, top and flow adapters adjusted to create an effective bed height of $0 \mathrm{~mm}$.

\subsubsection{Methods}

\section{Column preparation and characterization}

The column was slurry packed in two steps. The first began with phosphate buffer to settle the slurry in a ramped up profile of up to $10 \mathrm{~mL} / \mathrm{min}$ for 20 minutes. In the second step the funnel for the slurry packing was removed, the flow adapter and a filter placed above the stationary phase bed and the stationary phase bed further compressed at $10 \mathrm{~mL} / \mathrm{min}$ for 30 minutes. External porosity was measured with $10 \mathrm{~g} / \mathrm{L}$ dextran with an average molecular weight of approximately 2,000,000 $\mathrm{Da}$ (for the purpose of clarity referred to as dextran $2 \cdot 10^{6}$ ), total porosity was measured with $\mathrm{D}_{2} \mathrm{O}$, except for the case of Sephadex G-10, where only acetone was available for total porosity determination. Comparison in the two other Sephadex stationary phases showed close similarity in retention volume for $\mathrm{D}_{2} \mathrm{O}$ and acetone. All porosity measurements were conducted in phosphate buffered mobile phase at $25^{\circ} \mathrm{C}$. For all experiments the same mobile phase was used and no adsorption took place. Therefore, the particle porosity was assumed to remain constant for each stationary phase throughout this work. External porosity was confirmed by comparison of measured pressure drop over the column bed with the estimated pressure drop, calculated with the Ergun equation [61].

\section{Chromatographic analysis}

All chromatographic measurements were conducted as pulse injections of $80 \mu \mathrm{L}$. The column was kept at $25^{\circ} \mathrm{C}$ through a water jacket. All peaks were analyzed with the method of moments in Microsoft Excel as described in [62]. Integration limits were set automatically at $1 \%$ of total peak height and baseline drift was corrected for automatically, where necessary, to mitigate common concerns of inaccuracy when using the method of moments [63-65]. Van Deemter curves were recorded at linear superficial velocities $u_{S}$ of 0.5, 1,2, and $3 \mathrm{~m} / \mathrm{h}$. Sephadex G-25 was additionally measured at $u_{S}=0.2 \mathrm{~m} / \mathrm{h}$, the Toyopearl stationary phases were additionally measured at $u_{S}=4 \mathrm{~m} / \mathrm{h}$. All measurements were corrected for the extra-column contribution for each mobile phase velocity and target molecule, with the zero length column as described in [62]. For comparison of data from different stationary phases and target molecules, van Deemter curves were normalized by dividing HETP by the resin particle diameter $d_{p}$, which yields the reduced HETP $h$ and the linear interstitial velocity $u_{L}$ is multiplied by $d_{p}$ and divided by $D_{m}$ which yields the reduced velocity $v$.

\section{Bulk diffusion coefficient}

The bulk diffusion coefficient $D_{m}$ of $\mathrm{D}_{2} \mathrm{O}$ was taken from [66]. Bulk diffusion coefficients of all other molecules were calculated with the correlation of Wilke and Chang, with molecular volumes calculated from the correlation of LeBas, both as described in [67]. For the estimated bulk diffusion coefficient an error of $20 \%$ was assumed. 


\section{Measuring intraparticle diffusivity}

Intraparticle diffusivity was measured by fitting the plate height equation of the lumped kinetic model to experimental van Deemter curves, based on [27], assuming a constant and homogenous distribution of $\varepsilon_{p}$. The slope was measured from the linear region of four point van Deemter curves (five measurement points for Sephadex G-25 and for the Toyopearl series) of HETP (m) over interstitial linear velocity $u_{L}(\mathrm{~m} / \mathrm{s})$. From the slopes of the van Deemter curves the lumped kinetic factor $k_{\text {overall }}$ was calculated with equation 3.9.

$$
k_{\text {overall }}=\frac{\frac{2}{\frac{1-\varepsilon_{b}}{\varepsilon_{b}}} \cdot\left(\frac{k_{1}}{1+k_{1}}\right)^{2}}{\left(\frac{H E T P}{u_{L}}\right)}
$$

In size exclusion chromatography, the zone retention factor $k_{l}$ is dependent on a molecule's ability to penetrate pore volume, rather than adsorption equilibria, therefore $\varepsilon_{p . S E C}$ is used in equation 3.10 , based on [42].

$$
k_{1}=\frac{1-\varepsilon_{b}}{\varepsilon_{b}} \cdot \varepsilon_{p . S E C}=\frac{1-\varepsilon_{b}}{\varepsilon_{b}} \cdot \frac{V_{R}-V_{0}}{V_{C}-V_{0}}
$$

With the retention volume $V_{R}$, the void volume $V_{0}$ and the geometric column volume $V_{C}$. Intraparticle diffusivity $D_{p}$ was then calculated from equation 3.11 .

$$
D_{p}=\frac{r_{p}^{2}}{15\left(\frac{1}{k_{\text {overall }}}-\frac{r_{p}}{3 \cdot k_{\text {film }}}\right)}
$$

With $r_{p}$ particle radius and the resistance to mass transfer through the stagnant film layer $k_{f i l m}$, calculated as a function of reduced velocity $v=\left(2 \cdot r_{p} \cdot u_{L}\right) / D_{m}$ from the correlation of Wilson and Geankoplis [68] as shown in equation 3.12 .

$$
k_{f i l m}=\frac{1.09}{\varepsilon_{b}} \frac{D_{m}}{2 \cdot r_{p}} v^{1 / 3}
$$

This method relies on an assumed linearity for the calculation of a constant $k_{\text {overall }}$ for the entire linear region of the van Deemter curve. However, since $k_{\text {overall }}$ is a function of linear velocity, as it is dependent on $k_{\text {film }}$, the van Deemter curve is not truly linear. We therefore calculated $D_{p}$ for each measurement point of the curve and used the average of the calculated values for each van Deemter curve. The relative standard deviation of the $D_{p}$ measurements was just below $2 \%$ for all data points.

The confidence interval of $D_{p}$ was calculated from the propagated uncertainties of the slope and $k_{f i m}$. The uncertainty of the slope was calculated from the standard error of the slope with a $95 \%$ confidence interval and the uncertainty of $k_{\text {film }}$ from an uncertainty of $20 \%$ for $D_{m}$.

\section{Pore size distribution measurement}

The apparent pore size distribution was measured via inverse size exclusion chromatography, based on a lognormal pore size distribution as explained in [69]. The partition coefficient $K_{D}$ was calculated from the 
first moment of pulse injections for the target molecules listed in Table 3.2, using the mean retention volume $V_{R}$, the interparticle void volume $V_{0}$ and the total mobile phase volume $V_{T}$ (equation 3.13). Interparticle void volume and total mobile phase volume were measured with dextran $2 \cdot 10^{6}$ and $\mathrm{D}_{2} \mathrm{O}$ respectively.

$$
K_{D}=\frac{V_{R}-V_{0}}{V_{T}-V_{0}}
$$

Equation 3.14 was fitted to the plot of $K_{D}$ over molecular radius $r_{m}$ for each stationary phase using gProms Modelbuilder 4.0. Fitting parameters were $r_{p}$ and $s_{p}$ of the pore size distribution function $f(r)$ in equation 3.15. The pore shape dependent constant $a$ was assumed to be 2 (cylindrical pores), as discussed in [70].

$$
K_{D}=\frac{\int_{r_{m}}^{\infty} f(r)\left[1-\left(r_{m} / r\right)\right]^{a} d r}{\int_{0}^{\infty} f(r) d r}
$$

The function $f(r)$ in equation 3.15 describes the pore size distribution as a log-normal probability density function. The fitting parameters $r_{p}$ and $s_{p}$ are the mean and standard deviation of the distribution, respectively [71].

$$
f(r)=\frac{1}{r \sqrt{2 \pi}}\left[\ln \left(1+\left(\frac{s_{p}}{r_{p}}\right)^{2}\right)\right]^{-0.5} \cdot e^{\left[-\frac{\left(\ln \left(\frac{r}{r_{p}} \cdot\left[1+\left(\frac{s_{p}}{r_{p}}\right)^{2}\right]^{0.5}\right)\right)^{2}}{2 \cdot \ln \left(1+\left(\frac{s_{p}}{r_{p}}\right)^{2}\right)}\right]}
$$

From the fitted function the $K_{D}$ curve was calculated and the predicted $K_{D}$ used to describe the accessible pore fraction of pore volume for each molecule based on its size.

\section{Contributions to the internal obstruction factor}

Tortuosity was measured via electric impedance in phosphate buffer, based on [46] and [47]. All measurements were conducted at room temperature in a conductivity cell with a Vertex $10 \mathrm{~A}$ impedance analyzer and IviumSoft software (both by Ivium technologies, The Netherlands). Impedances were measured in phosphate buffer without stationary phase particles and in phosphate buffer with stationary phase particles sedimented into the upside-down conductivity cell. The exact value of the external porosity in the conductivity cell was not known. Bed porosity was estimated to be slightly larger than the geometric optimum of 0.34 . We therefore calculated tortuosity for five different bed porosities in range of 0.36 through 0.44 and worked with the average value as well as the standard deviation. With equation 3.16 the total tortuosity $\tau_{t}$ was calculated from the measured impedance in sedimented stationary phase $\sigma_{t}$ and without stationary phase $\sigma_{0}$.

$$
\frac{\sigma_{0} \cdot \varepsilon_{t}}{\sigma_{t}}=\tau_{t}
$$

Intraparticle tortuosity was derived from particle conductivity with equation 3.17 [47]. 


$$
\varepsilon_{t} \cdot \frac{2+\frac{\sigma_{p}}{\sigma_{0}}+\left(1-\varepsilon_{e}\right) \cdot\left(1-\frac{\sigma_{p}}{\sigma_{0}}\right)}{2+\frac{\sigma_{p}}{\sigma_{0}}-2 \cdot\left(1-\varepsilon_{e}\right) \cdot\left(1-\frac{\sigma_{p}}{\sigma_{0}}\right)}=\tau_{t}
$$

Using the solver add-on in Microsoft Excel, the intraparticle conductivity $\sigma_{p}$ was fitted in equation 3.17, particle tortuosity $\tau_{p}$ was then calculated with equation 3.18 .

$$
\frac{\sigma_{0} \cdot \varepsilon_{p}}{\sigma_{p}}=\tau_{p}
$$

As pointed out in section 3.2.5, validation of the obstruction to diffusion due to constriction $\gamma_{p, c o n s}$ and connectivity $\gamma_{p, c o n n}$ cannot be isolated and validated in practice. For the contribution of constriction and connectivity to the internal obstruction factor $\gamma_{p}$, the authors therefore resigned to a value of 1 in equation 3.5.

\section{Visualization of stationary phases and pore structures}

Two electron microscopy methods were used to visualize the presence of the pores: focused ion beam - scanning electron microscopy FIB-SEM and transmission electron microscopy TEM. Small amounts of the stationary phases were oven-dried overnight at $60^{\circ} \mathrm{C}$. The resulting powder was subsequently sprinkled onto a standard aluminum SEM stub with a carbon sticker on top. Following, a metallic layer $P t$ was sputter coated (Cressington, HQ280) across the stub to ensure sufficient electrical conduction.

The FIB-SEM (Thermo Scientific, Helios Nanolab G3-UC) combines the imaging capabilities of the SEM with the milling capabilities of a $F I B$. The $F I B$ is a beam of gallium ions which scans the surface of a sample. The momentum transfer of the gallium ions onto a sample causes the samples atoms to disappear into the vacuum, a process called sputtering or milling. Prolonged milling results in a trench or cross section of some tens of micro meters. Subsequently, the SEM is employed to visualize the cross section. Visualization is done in backscatter electron mode, which is less affected by local surface charge.

Milling and imaging was performed at customary conditions: a $30 \mathrm{keV}$ ion beam, starting at $9.4 \mathrm{nA}$ and gradually reducing to $40 \mathrm{pA}$ for the final polishing. Prior to the milling, a small layer $(1 \mu \mathrm{m})$ of $P t$ was deposited across the region of interest. The $P t$ deposition acts as protection against the ion beam and it smoothens the surface and therefore the finish of the cross section. Imaging polymeric samples with electron microscopy is not trivial. The low atomic weight of the polymer chains doesn't create any contrast.

The TEM analyzed Dowex 50WX2 sample was stained with $0.1 \mathrm{~mL} / \mathrm{g} \mathrm{FeSO}_{4}$. An additional challenge is the resolving power of the $S E M$. An ideal sample can be resolved down to $0.8 \mathrm{~nm}$. However, the resolving power obtained from unstained polymers is probably not better than $10 \mathrm{~nm}$. Therefore, pores $>10 \mathrm{~nm}$ can be investigated directly by FIB-SEM. In addition, the presence of $1-2 \mathrm{~nm}$ pores was therefore investigated by transmission electron microscopy TEM. TEM requires a thin sample of no more than $100 \mathrm{~nm}$ thick, which were made by the FIB-SEM. Again standard procedures were followed. The final polishing step was done at $30 \mathrm{kV}, 40 \mathrm{pA}$. The TEM (Thermo Scientific, Talos F200x) in STEM mode, using the High Angular Annular Dark Field $H A A D F$ detector. 
Note on availability of data

In an effort to support the understanding of intraparticle diffusivity and its relation to stationary phase characteristics, all of the measured data is made available in the supplementary material of this manuscript.

\subsection{Results and discussion}

\subsubsection{Intraparticle diffusion}

Intraparticle diffusion was measured in nine different stationary phases with eight different tracer molecules at the same conditions (Fig. 3.1). Data in Fig. 3.1 is grouped per backbone material, within each backbone material per decreasing cross-linking and increasing molecular size, both left to right. Determination via the slope of van Deemter curves gave accurate results, the majority of the error bar seen in Fig. 3.1 is due to the uncertainty of $20 \%$ allocated to the bulk diffusion coefficient $D_{m}$ estimated with the Wilke-Chang equation. As expected, intraparticle diffusion, conveniently expressed as dimensionless ratio of intraparticle to bulk diffusion $D_{p} / D_{m}$, differs from stationary phase to phase and molecule to molecule. All experimental van Deemter curves can be found in the supplementary material (Fig. 3.8, Fig. 3.9, and Fig. 3.10). All elution data can be found in Table 3.6 in the supplementary material.

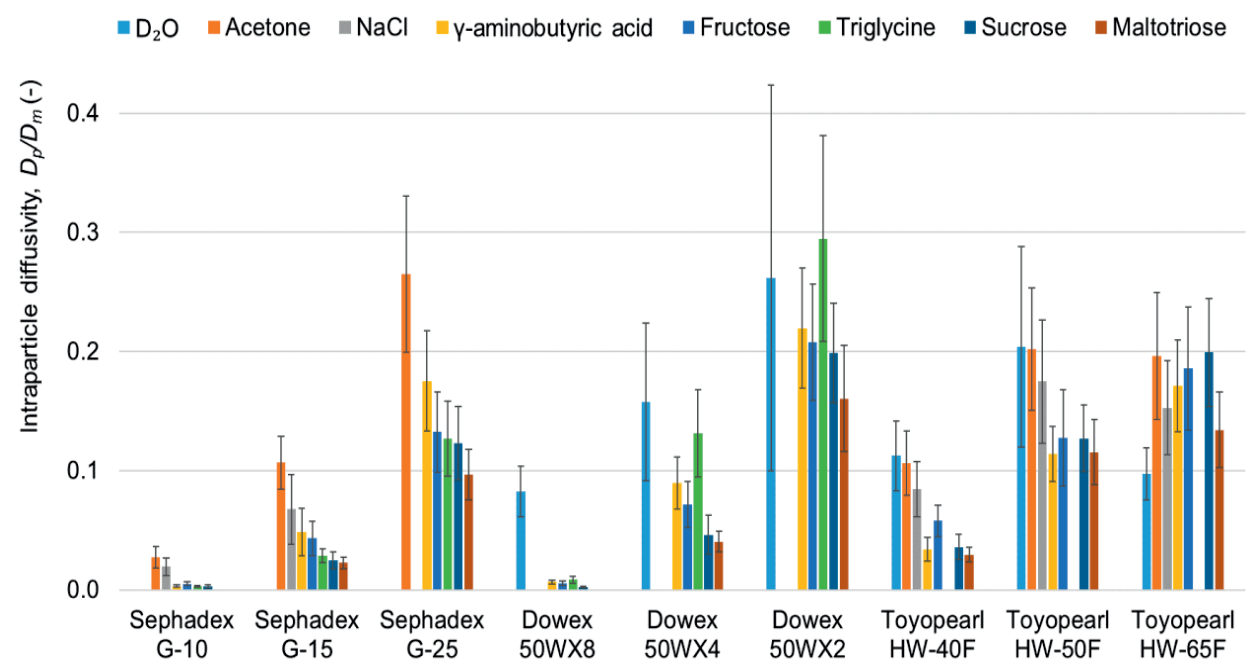

Fig. 3.1 - Measured intraparticle diffusion ratio $D_{p} / D_{m}$ in all nine stationary phases for all target molecules. Error bars indicate uncertainty of determination of $D_{p}$ from slope of van Deemter curves (based on a $95 \%$ confidence interval) and $20 \%$ uncertainty of $D_{m}$ estimation.

Two trends are obvious in the Sephadex stationary phases: first, decreased cross-linking has a positive effect on intraparticle diffusivity and second, increasing target molecule size decreased intraparticle diffusivity. Both observations are easily explained by the mass transfer limiting mechanisms, where smaller molecules experience less resistance to diffusion than larger molecules and pore dimensions increase with decreasing 
cross-linking. The Dowex series, a cation exchange material, shows a similar trend in relation to the crosslinking. The same correlation with the target molecule size holds, with the exception of triglycine. Finally, in the Toyopearl series most of the correlations between intraparticle diffusivity, cross-linking and target molecule size are lost. Toyopearl HW-50F and HW-65F showed comparable measured intraparticle diffusivities. According to the manufacturer, the pore size of Toyopearl HW-65F is eight times larger than for HW-50F and 20 times larger than for HW-40F, a difference in pore size which was not apparent from the measured data.

Perhaps most remarkable is the relatively low intraparticle diffusivity of $\mathrm{D}_{2} \mathrm{O}$ in comparison to larger molecules. In order to explain the observations in Fig 1, additional information regarding the pore structure is required.

\subsubsection{Particle size distribution and porosity}

The Sauter diameter was measured in ten consecutive measurements in the Mastersizer. The average values along with the measured relative standard deviations are given for each stationary phase in Table 3.3. Additionally $\mathrm{d}_{10}$ and $\mathrm{d}_{90}$ (the diameters where $10 \%$ and $90 \%$ of the distribution has a larger particle size respectively), are added given in Table 3.3, along with their respective relative standard deviations. It was not possible to obtain all stationary phases of a series with the same particle diameter, however influence of particle size on mass transfer resistance was accounted for (an input parameter in the modelling equations (e.g. equations 3.11 and 3.12) and by normalizing the van Deemter curves). Particle diameter was additionally measured from SEM images, in the following referred to as $d_{S E M}$, by averaging at least 35 particles. The Sauter diameter and $d_{S E M}$ differ substantially. It is likely that the particles shrank upon drying or in the vacuum chamber, as the stationary phase had not been fixated. Consequently, pore structures may have changed.

Table 3.3 - Stationary phase series, Sauter diameter, $d_{10}$ and $d_{90}$, and their relative standard deviations for all stationary phases. The particle diameter $d_{\text {SEM }}$ was determined from electron microscopy images. Additionally measured particle porosities and apparent mean pore sizes (from ISEC measurements as detailed in section 0 ).

\begin{tabular}{|c|c|c|c|c|c|c|}
\hline Stationary phase & $\begin{array}{c}\text { Sauter diameter } \\
{[\mu \mathrm{m}]}\end{array}$ & $d_{10}$ & $d_{90}$ & $\begin{array}{l}d_{S E M} \\
{[\mu m]}\end{array}$ & $\begin{array}{c}\text { Particle } \\
\text { porosity, } \varepsilon_{p}\end{array}$ & $\begin{array}{c}\text { Apparent mean } \\
\text { pore size }[\mathrm{nm}]\end{array}$ \\
\hline Sephadex $G-10$ & $88 \pm 0.8 \%$ & $65 \pm 1.0 \%$ & $125 \pm 0.5 \%$ & n.d. & 0.46 & 1.0 \\
\hline Sephadex $G-15$ & $74 \pm 0.2 \%$ & $55 \pm 0.4 \%$ & $110 \pm 0.2 \%$ & 58 & 0.66 & 1.4 \\
\hline Sephadex $G-25$ & $262 \pm 1.1 \%$ & $91 \pm 1.2 \%$ & $400 \pm 0.8 \%$ & n.d. & 0.73 & 1.7 \\
\hline Dowex 50WX8 & $91 \pm 0.1 \%$ & $72 \pm 0.7 \%$ & $121 \pm 0.6 \%$ & 71 & 0.52 & 0.7 \\
\hline Dowex 50WX4 & $106 \pm 0.3 \%$ & $82 \pm 0.4 \%$ & $142 \pm 0.2 \%$ & n.d. & 0.68 & 1.4 \\
\hline Dowex 50WX2 & $141 \pm 0.8 \%$ & $11 \pm 0.8 \%$ & $186 \pm 1.1 \%$ & 64 & 0.84 & 2.3 \\
\hline Toyopearl HW-40F & $48 \pm 0.4 \%$ & $39 \pm 1.2 \%$ & $62 \pm 0.9 \%$ & n.d. & 0.66 & 1.7 \\
\hline Toyopearl HW-50F & $50 \pm 0.1 \%$ & $39 \pm 0.1 \%$ & $66 \pm 0.1 \%$ & 34 & 0.72 & 5.0 \\
\hline Toyopearl HW-65F & $52 \pm 0.2 \%$ & $44 \pm 0.3 \%$ & $64 \pm 0.4 \%$ & 33 & 0.68 & 35.0 \\
\hline
\end{tabular}

The measured particle porosities varied between 0.46 in Sephadex G-10 and 0.84 in Dowex 50WX2 and increased with decreasing cross-linking within a series, except for Toyopearl HW-65F, which shows a slightly smaller porosity than Toyopearl HW-50F (Table 3.3). The particle porosity for Toyopearl HW-65F matches data reported in literature well [69]. 


\subsubsection{Visualization of pore structures}

In total five of the nine stationary phases were analyzed in a FIB-SEM (Sephadex G-15, Dowex 50WX8 and 50WX2, and Toyopearl HW-50F and HW-65F) and one in a TEM (Dowex 50WX2). Examples from the outside of particles and pore structures, laid bare with a focused ion beam, can be seen in Fig. 3.2. Visualizing pore structures proved to be very challenging due to the very small diameters. Only the Toyopearl HW-65F revealed a pore structure. The absence of macro pores (pore diameters exceeding $50 \mathrm{~nm}$ [41]) was the only conclusion that could be drawn for the other four stationary phases analyzed in FIB-SEM. High resolution TEM imaging was only just able to reveal structures in the Dowex 50WX2 sample. The presented electron microscopy data is inconclusive with respect to relating intraparticle diffusivity to pore structures, given the shrinkage of particle size compared to particle size distribution measurements in phosphate buffer (Table 3.3).
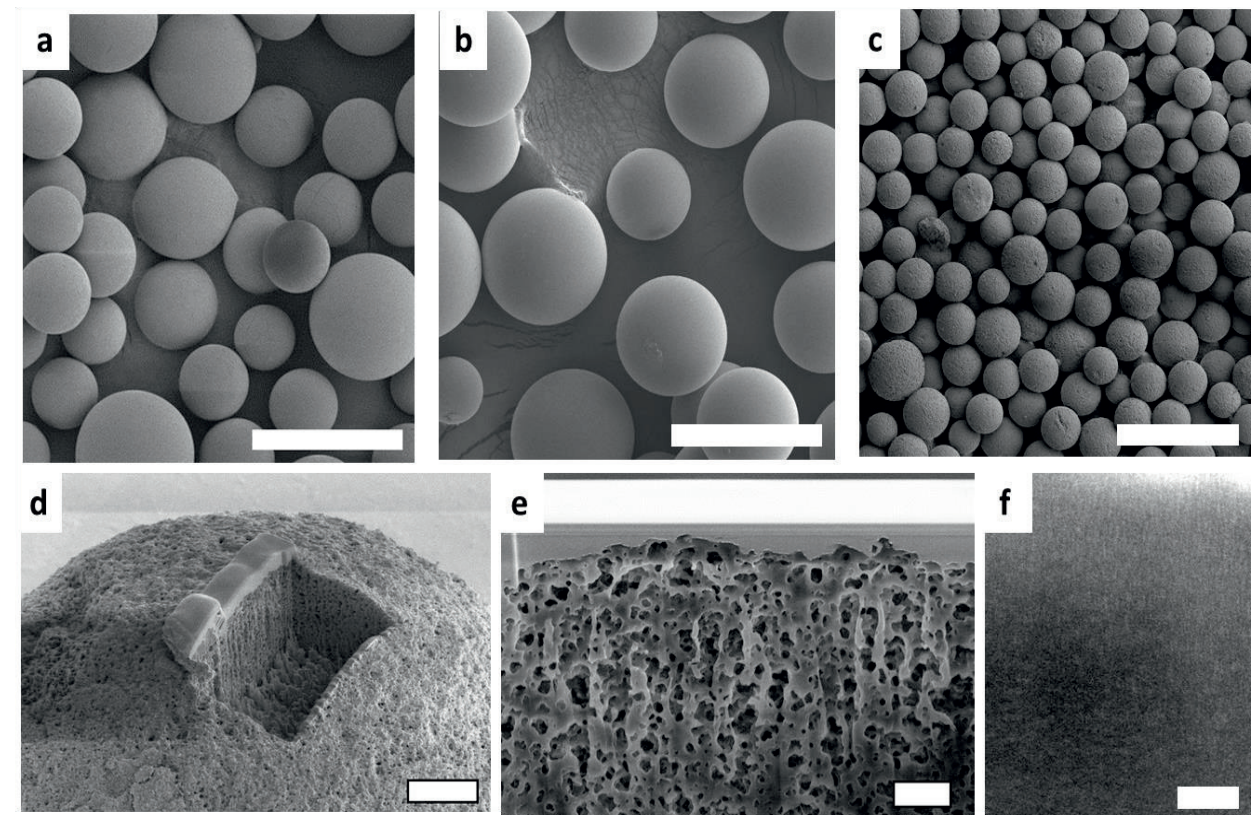

Fig. 3.2 - Examples from the stationary phase as examined by FIB-SEM and TEM: a) Sephadex G-15 b) Dowex $50 \mathrm{WX} 8$ c) Toyopearl HW-65F. d) A FIB cross section was made into an individual Toyopearl HW-65F particle and imaged e) by the SEM. The pore dimensions of the other stationary phases are of the order of 1-2 nm and can only just be made visible by TEM (f, Dowex 50WX2). Scale bars are (a-c) $100 \mu \mathrm{m}, \mathrm{d}) 5 \mu \mathrm{m}$, e) $1 \mu \mathrm{m}$ and f) $40 \mathrm{~nm}$.

\subsubsection{The correlation of Mackie and Meares}

The correlation of Mackie and Meares uses particle porosity as sole parameter to determine intraparticle diffusivity. It is important to note the role of particle porosity, as measurement with a different molecule yields very different results. A smaller molecule will have access to a different pore volume than a larger molecule $[69,72]$. In this study the smallest readily available molecule, $\mathrm{D}_{2} \mathrm{O}$, was used for the determination of the total and particle porosity. Other studies which used same method to measure particle porosity used different 
molecules like a monomeric sugar, e.g. [69]. For illustration purposes, we also calculated total and particle porosity based on the retention of fructose. Fructose has roughly three times the molecular radius of heavy water. Fig. 3.3a and Fig 3b plot the normalized intraparticle diffusivities as a function of particle porosity, based on the retention of $\mathrm{D}_{2} \mathrm{O}$ and fructose respectively. The dashed line indicates the Mackie and Meares correlation. The experimental results follow the expected boundaries to diffusion in porous space, as discussed in section 3.2.2. However, the correlation systematically over-estimates the diffusivity values, when particle porosity is based on the retention of $\mathrm{D}_{2} \mathrm{O}$. Calculated particle porosities are on average $30 \%$ smaller, when particle porosity is based on the retention of fructose. In consequence measured intraparticle diffusivities match the correlation of Mackie and Meares visibly better, albeit far from perfect. This result is of little practical relevance, but it serves to emphasize the importance of $\varepsilon_{t}$ and $\varepsilon_{p}$ determination. We suggest the use of $\mathrm{D}_{2} \mathrm{O}$ for particle porosity measurements, as it measures a more relevant pore spectrum for the chromatographic separation of small target molecules, such as small sugars and peptides. In all following calculations $\varepsilon_{t}$ and $\varepsilon_{p}$ are based on the retention of $\mathrm{D}_{2} \mathrm{O}$.
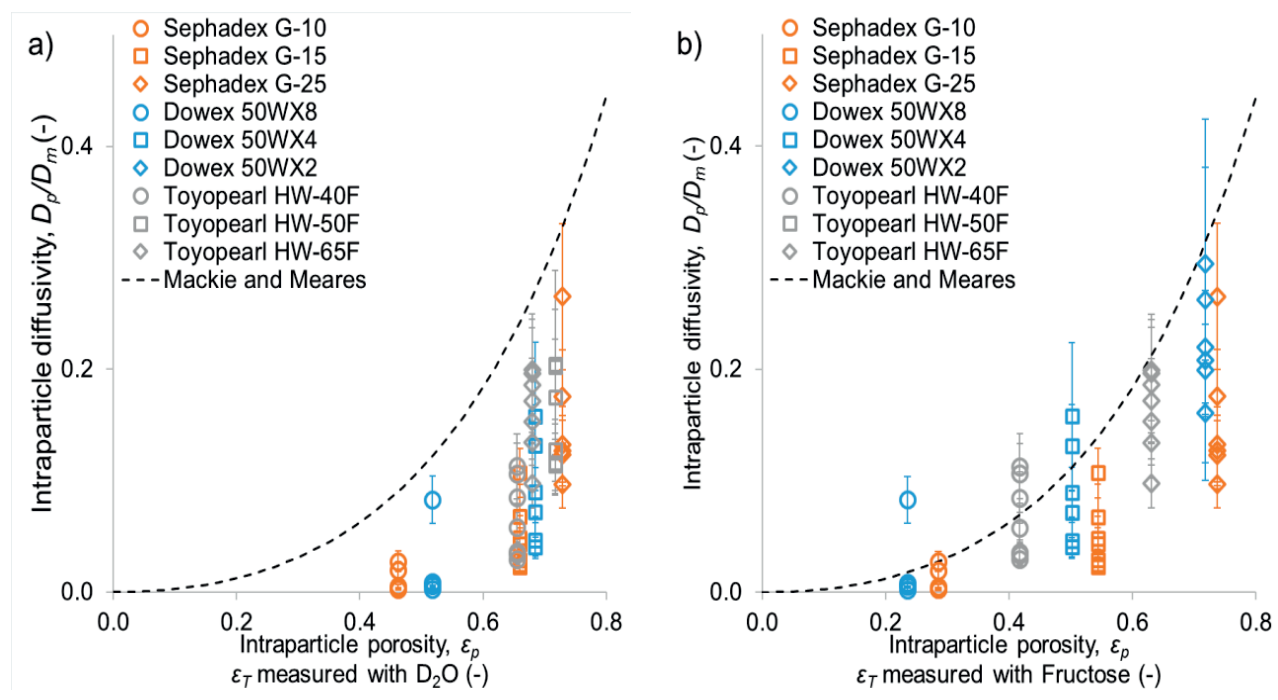

Fig. 3.3 - Intraparticle diffusion as function of particle porosity $\varepsilon_{p}$ for different molecules in nine different stationary phases and the correlation of Mackie and Meares (dotted line). a) $\varepsilon_{p}$ is based on retention of $\mathrm{D}_{2} \mathrm{O}$ and dextran, b) $\varepsilon_{p}$ is based on retention of fructose and dextran.

The correlation of Mackie and Meares may serve as an early estimation of intraparticle diffusivity, but low accuracy must be assumed. From Fig. 3.3a can be observed that particle porosity alone is insufficient as parameter to predict intraparticle diffusivity. This is clearly reflected in the vertical distribution of intraparticle diffusivity values in Fig. 3.3a. A single particle porosity value can produce a range of diffusivity values, even after normalization. Additional structural properties of both the stationary phase and the target molecules are not considered. 


\subsubsection{Apparent pore size distribution}

For the measurement of pore size distribution, $K_{D}$ curves were recorded for each stationary phase, depicting the accessible fraction of pore volume for molecules of different sizes (closed symbols in Fig. 3.4a, b, and c). Lognormal pore size distribution curves were fitted to the experimental data. Based on the underlying function (equation 3.14) the $K_{D}$ curves were calculated (lines in Fig. 3.4a, b, and c). Note, Fig. 3.4a, b, and c each have a differently scaled y-axis to accommodate different pore size distributions. In general, the fitting led to a good description of the experimental data. However, for none of the resins the pore size distribution $f(r)$ of equation 3.15 could describe the $\mathrm{D}_{2} \mathrm{O}$ data point $\left(K_{D}=1, r_{m}=0.09 \mathrm{~nm}\right)$. This is due to the fact that the finite size of the molecule leads to a reduction to the fraction of accessible pore volume. The small mean pore sizes fitted (Table 3.4) resulted even for $\mathrm{D}_{2} \mathrm{O}$ in $K_{D}<1$. It was not possible to determine the standard deviation of the pore size distribution. The fitted function is sensible to variance only in the range of very small $K_{D}$ values, for $K_{D} \geq 0.2$ different variances are barely discernible in the function.
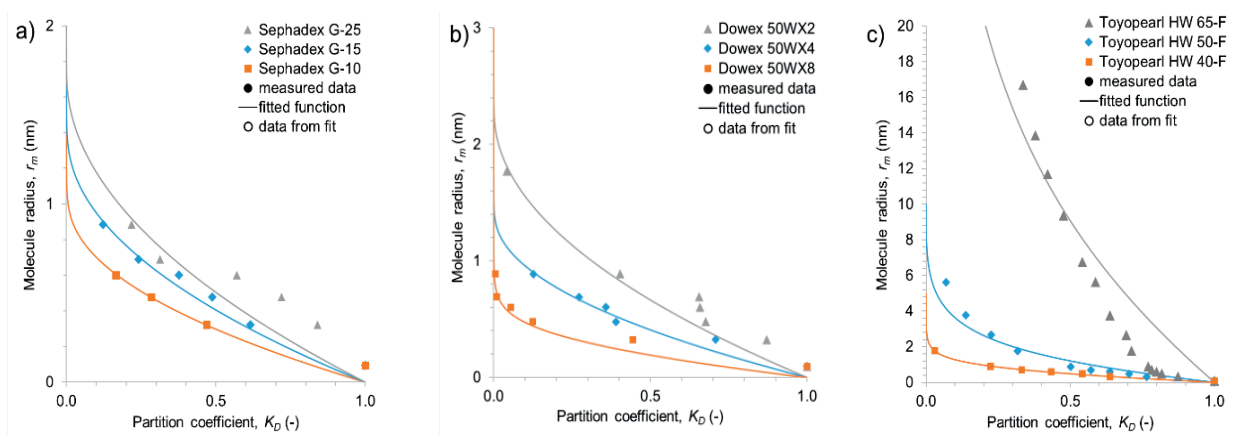

Fig. $3.4-K_{D}$ curves of a) Sephadex, b) Dowex and c) Toyopearl stationary phases, relating the partition coefficient to molecular radii. Measurements (symbols) and fitted functions (solid lines). Due to the larger pores, also larger molecules were employed for the pore size measurement of the Toyopearl series, therefore the y-axis is scaled to a different maximum.

All data recorded during inverted size exclusion measurements can be found in Table 3.15, Table 3.16, and Table 3.17 in the supplementary material.

Table 3.4 - Fitted mean pore radii of pore size distribution for each stationary phase.

\begin{tabular}{r|c} 
Stationary phase & Mean pore radius, $\boldsymbol{r}_{\boldsymbol{p}}(\mathbf{n m})$ \\
\hline Sephadex $G-10$ & 1.0 \\
Sephadex $G-15$ & 1.4 \\
Sephadex $G-25$ & 1.7 \\
Dowex 50WX8 & 0.7 \\
Dowex 50WX4 & 1.4 \\
Dowex 50WX2 & 2.3 \\
Toyopearl HW-40F & 1.7 \\
Toyopearl HW-50F & 5.0 \\
Toyopearl HW-65F & 35.0
\end{tabular}


The fitted mean pore sizes correlate well to measured intraparticle diffusion data of section 3.4.1. The Sephadex material shows a consistent correlation: larger pores result in higher intraparticle diffusivity. The same correlation is found for the Dowex series. The difference in mean pore sizes for the Toyopearl series is more pronounced. Both, in comparison to the other two backbone materials, as well as the difference between Toyopearl HW-F40/F50 and Toyopearl HW-F65. Both observations are not reflected in the measured intraparticle diffusivity. For all nine stationary phases the mean of the pore size distribution increases with decreasing cross-linking.

Pore size distribution measurement via inverted size exclusion chromatography ISEC does not yield absolute but functional values and resulting data should be referred to as apparent pore size distribution [70]. This is partly due to a pore shape parameter within the fitting function ( $a$ in equation 3.14), which requires an assumption about the pore shape [70], although it has been later shown that ISEC is fairly insensitive to the descriptions of pore geometry [13]. Especially in gels, where pores and pore structures are somewhat differently defined, pore size distribution measurement via ISEC is mainly of functional use, rather than matching the geometry of the gel [73] and can only be used to simplify description of pores in gels [74].

The Toyopearl stationary phase series are the only series for which pore sizes are provided by the manufacturer, however the reference does not include the measurement method for the pore radii [75]. The pore radii are 2.5, 6.3, and $50 \mathrm{~nm}$ for the Toyopearl HW40-F, HW50-F, and HW-65F respectively, the latter was also found by [69]. Mean pore radii measured in this work for the Toyopearl series value about 70 to $80 \%$ of the data supplied by the manufacturer, although the fitted $K_{D}$ curves of Toyopearl HW40-F and HW50-F in Fig. 3.4 match measured data reasonably well. The different result highlights how much the results depend on the method used to acquire the data.

Toyopearl HW65-F is the only stationary phase analyzed in this work with observable macropores from SEM analysis. The viscosity radius of the largest molecule employed in this research, a dextran molecule of approximately $2,000,000 \mathrm{Da}$, is $37 \mathrm{~nm}$. Thus it is likely that the dextran molecule is capable of accessing a fraction of the macro-porous pore space, which yields the measurement of external porosity inaccurate. This affects the accuracy of both of intraparticle diffusivity and measured pore size distribution as well. An even larger molecule to measure external porosity, for example large DNA molecules as used in [69], would certainly not be able to penetrate any pore space.

\subsubsection{Obstruction due to tortuosity}

Particle tortuosity, measured via electric impedance, shows trends within each stationary phase series, that correlate to particle porosity. With increasing particle porosity, tortuosity decreases, and the obstruction due to tortuosity $\gamma_{p, \tau}$ increases, just as predicted in literature, e.g. [21]. External porosity is unknown, but a required input factor in equation 3.17. The results in Fig. 3.5 show the average of the obstruction due to tortuosity $\gamma_{p, \tau}$, calculated for five assumed external porosities, as detailed in section 3.3.2 Contributions to the internal obstruction factor, with the error bar as standard deviation of the five results. At similar particle porosity, the tortuosities of Sephadex and Toyopearl stationary phases are very similar. The Dowex stationary phase series shows the largest $\gamma_{p, \tau}$, which may be due to the fact that the ionic surface charge on the ion-exchange stationary phase reduces impedance. Measured obstruction factors can be found in Table 3.18 in the supplementary material. 


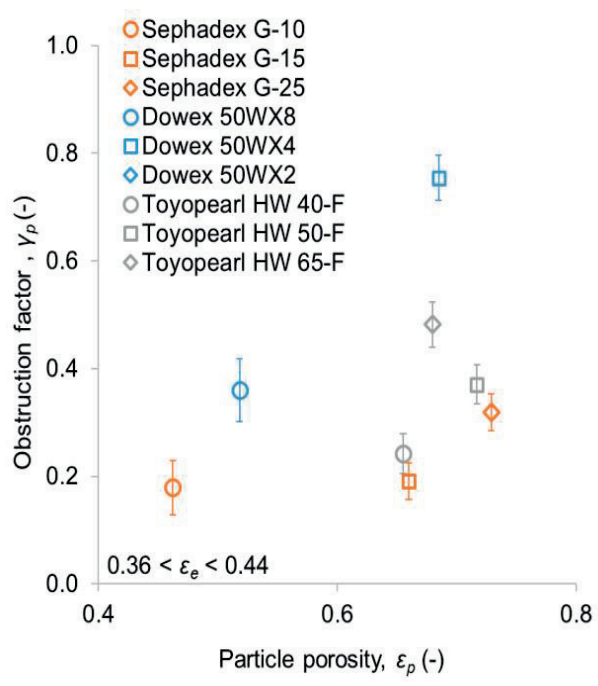

Fig. 3.5 - Obstruction due to tortuosity calculated from particle tortuosity measured via electric impedance. Exact external porosities were unknown, therefore tortuosity was calculated for five estimated external porosities between 0.36 and 0.44 . Displayed value is the average of five calculations with the standard deviation as the error bar.

\subsubsection{The parallel pore model}

Correlating intraparticle diffusion to individual stationary phase properties, as defined in the parallel pore model, in combination with properties of the diffusing molecules did not lead to a conclusive correlation. In Fig. 3.6 we show the correlation of measured intraparticle diffusivities to the product of particle porosity, hindrance to diffusion, and internal obstruction factor, the parallel pore model.

Bringing together all three parameters of the parallel pore model in the relation to measured intraparticle diffusivity lead to reasonably accurate predictions for small intraparticle diffusivities $\left(D_{p} / D_{m}<0.2\right)$, with the exception of Sephadex G-25. The data appeared to "level off" for larger intraparticle diffusivities. Generally, intraparticle diffusion in the Sephadex series appeared to be underestimated, while the Dowex and Toyopearl data appeared to be overestimated.

In comparison to the simple correlation of Mackie and Meares (Fig. 3a), the parallel pore model is an improvement. It provides more insight into the interplay of geometric properties between stationary phase and diffusing molecule and the predictability of the intraparticle diffusivity increases. However, based on Fig. 3.6 it is not possible to predict the intraparticle diffusivity over the whole measurement range, even though the parallel pore model considers more data. We have attempted to find an explicit correlation between intraparticle diffusivity and pore characteristics, but not all model input parameters were experimentally measurable. It is possible that pore structure is a topic more complex than captured in the three parameters of the parallel pore model. 


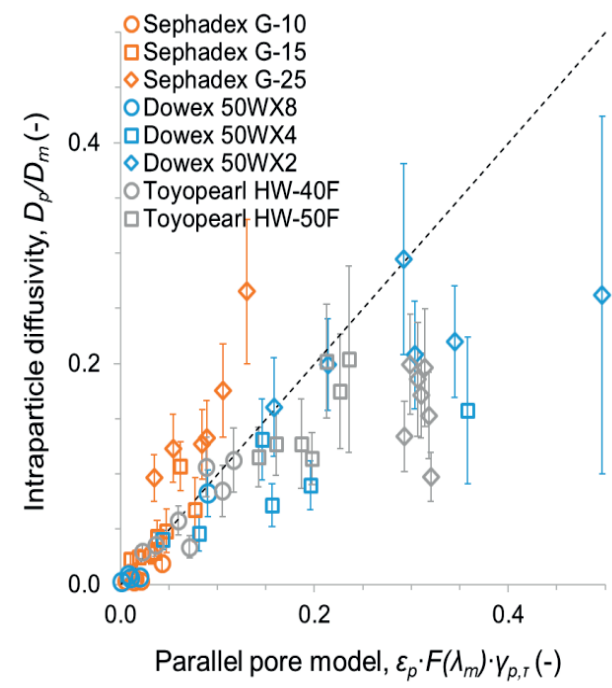

Fig. 3.6 - Correlation of measured intraparticle diffusivity to the parallel pore model: product of particle porosity $\varepsilon_{p}$, hindrance diffusion factor $F\left(\lambda_{m}\right)$, and internal obstruction factor $\gamma_{p, \tau}$.

\subsubsection{Accessible fraction of pore volume and its influence on intraparticle diffusivity}

In an attempt to relate measured intraparticle diffusivities more accurately to pore characteristics, the accessible fraction of pore volume for each molecule was calculated from the product of $K_{D}$ and particle porosity $\varepsilon_{p}$. Here, $K_{D}$ was calculated with equation 3.14 for each molecule and stationary phase. Plotted against measured intraparticle diffusivity the accessible fraction of pore volume yields an exponential trend that follows the trend predicted by Mackie and Meares (Fig. 3.7), which in this case should be interpreted according to equation 3.19 .

$$
D_{P}=\left[\frac{K_{D} \varepsilon_{P}}{2-K_{D} \varepsilon_{P}}\right]^{2} D_{m}
$$

This method yields a clear correlation between measured intraparticle diffusivities and pore characteristics and provides a predictive model. The main advantage of this predictive model is that it relies only on ISEC measurements that can be collected from a packed column, in which the stationary phase is in the same conditions as during the anticipated separation process. Furthermore, the use of the accessible fraction of pore volume does not rely on absolute pore dimensions, as it relies on data recorded with the same or similar molecules. The proposed equation should be further challenged, specifically widening the applicability to higher accessible pore fractions (> 0.7) with corresponding higher intraparticle diffusivities $\left(D_{p} / D_{m}>0.2\right)$. 


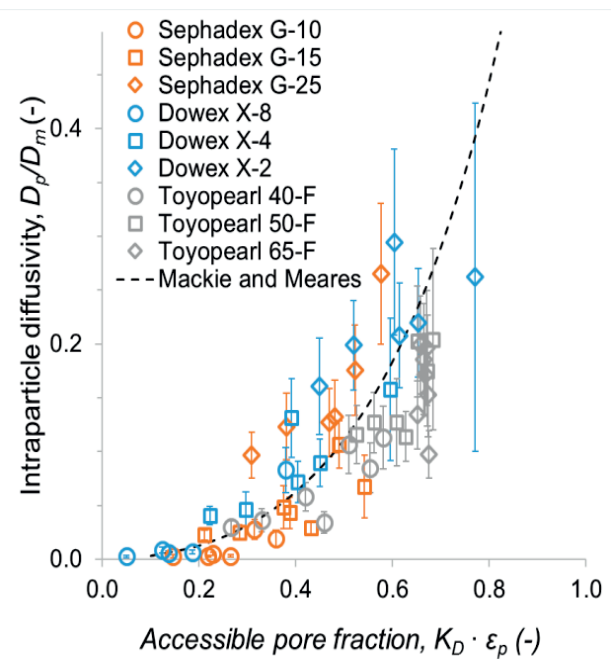

Fig. 3.7 - Accessible fraction of pore volume, calculated from the product of $K_{D}$ and particle porosity.

\subsection{Conclusions}

Measured intraparticle diffusivity $\left(D_{p} / D_{m}\right)$ in this work ranged from 0.02 to 0.2 , with a few exceptions. If a first estimate is required, it seems reasonable to assume diffusion inside a porous chromatographic particle to be around $10 \%$ of the bulk diffusion, as suggested by Nicoud [16] and Ruthven [21]. When the particle porosity is known, a better estimate is obtained with the Mackie and Meares correlation. Although, on average, it overestimates intraparticle diffusivity by a factor of three. Including further characterization of the resin by measuring the mean pore size, the internal obstruction factor and the hindrance diffusion factor, the parallel pore model can provide a better insight and prediction of the intraparticle diffusivity. However, the best prediction of the intraparticle diffusivity to stationary phase characteristics was obtained by using the Mackie and Meares correlation in combination with the apparent fraction of accessible pore volume. This approach should be further challenged, specifically widening the applicability to higher accessible pore fractions $(>0.7)$ with corresponding higher intraparticle diffusivities $\left(D_{p} / D_{m}>0.2\right)$.

\subsection{Acknowledgements}

The authors would like to thank Ronald Vroon for his input and help in this research as well as Loes van Ooijen and Bas Ooteman for their dedication and work on this project. This research took place within the framework of the Institute for Sustainable Process Technology ISPT. The authors would like to thank the ISPT for their support, together with Unilever (Vlaardingen, NL), FrieslandCampina Research (Amersfoort, NL), DSM (Delft, NL) and Cosun Food Technology (Roosendaal, NL) for their financial support and interest in this project. Hans Meeldijk is acknowledged for the TEM observations. Matthijs de Winter is supported by 
the Deutsche Forschungsgemeinschaft (DFG, German Research Foundation) - project number 327154368 SFB1313.

\subsection{References}

1. V.E. Barsky, A.M. Kolchinsky, Y.P. Lysov, A.D. Mirzabekov, Biological Microchips with HydrogelImmobilized Nucleic Acids, Proteins, and Other Compounds: Properties and Applications in Genomics, Molecular Biology, 36 (2002) 437-455.

2. T.-O. Peulen, K.J. Wilkinson, Diffusion of Nanoparticles in a Biofilm, Environmental Science \& Technology, 45 (2011) 3367-3373.

3. N. Murthy, Y.X. Thng, S. Schuck, M.C. Xu, J.M.J. Fréchet, A Novel Strategy for Encapsulation and Release of Proteins: Hydrogels and Microgels with Acid-Labile Acetal Cross-Linkers, Journal of the American Chemical Society, 124 (2002) 12398-12399.

4. S. Sershen, J. West, Implantable, polymeric systems for modulated drug delivery, Advanced Drug Delivery Reviews, 54 (2002) 1225-1235.

5. O. Wichterle, D. LÍM, Hydrophilic Gels for Biological Use, Nature, 185 (1960) 117-118.

6. H.-W. Kang, Y. Tabata, Y. Ikada, Fabrication of porous gelatin scaffolds for tissue engineering, Biomaterials, 20 (1999) 1339-1344.

7. A.S. Hoffman, Hydrogels for biomedical applications, Advanced Drug Delivery Reviews, 54 (2002) 3-12.

8. A.Z. Abidin, T. Puspasari, W.A. Nugroho, Polymers for Enhanced Oil Recovery Technology, Procedia Chemistry, 4 (2012) 11-16.

9. R. Zolfaghari, A.A. Katbab, J. Nabavizadeh, R.Y. Tabasi, M.H. Nejad, Preparation and characterization of nanocomposite hydrogels based on polyacrylamide for enhanced oil recovery applications, J. Appl. Polym. Sci., 100 (2006) 2096-2103.

10. D.A.M. de Winter, F. Meirer, B.M. Weckhuysen, FIB-SEM Tomography Probes the Mesoscale Pore Space of an Individual Catalytic Cracking Particle, ACS Catalysis, 6 (2016) 3158-3167.

11. P. Dechadilok, W.M. Deen, Hindrance Factors for Diffusion and Convection in Pores, Ind Eng Chem Res, 45 (2006) 6953-6959.

12. S.T. Balke, A.E. Hamielec, B.P. LeClair, S.L. Pearce, Gel Permeation Chromatography, Product R\&D, 8 (1969) 54-57.

13. Y. Yao, A.M. Lenhoff, Determination of pore size distributions of porous chromatographic adsorbents by inverse size-exclusion chromatography, Estimation of Physicochemical Properties by Chromatographic and Electrophoretic Techniques, 1037 (2004) 273-282.

14. L. Shen, Z. Chen, Critical review of the impact of tortuosity on diffusion, Chemical Engineering Science, 62 (2007) 3748-3755.

15. B. Ghanbarian, A.G. Hunt, R.P. Ewing, M. Sahimi, Tortuosity in Porous Media: A Critical Review, Soil Sci.Soc.Am.J., 77 (2013) 1461.

16. R.M. Nicoud, Chromatographic Processes, Cambridge University Press, 2015.

17. M. Schröder, E. von Lieres, J. Hubbuch, Direct Quantification of Intraparticle Protein Diffusion in Chromatographic Media, The Journal of Physical Chemistry B, 110 (2006) 1429-1436. 
18. G. Guiochon, D.G. Shirazi, A. Felinger, A.M. Katti, Fundamentals of Preparative and Nonlinear Chromatography, Academic Press, 2006.

19. D. Sandrin, D. Wagner, C.E. Sitta, R. Thoma, S. Felekyan, H.E. Hermes, C. Janiak, S.A. de, R. Kuhnemuth, H. Lowen, S.U. Egelhaaf, C.A.M. Seidel, Diffusion of macromolecules in a polymer hydrogel: from microscopic to macroscopic scales, Physical Chemistry Chemical Physics, 18 (2016) 12860-12876.

20. P. Dechadilok, W.M. Deen, Hindrance factors for diffusion and convection in pores, Industrial and Engineering Chemistry Research, 45 (2006) 6953-6959.

21. J. Kärger, D.M. Ruthven, D.N. Theodorou, Diffusion in Nanoporous Materials, 2 Volume Set, Wiley, 2012.

22. A.G. Ogston, B.N. Preston, J.D. Wells, On the Transport of Compact Particles Through Solutions of Chain-Polymers, Proceedings of the Royal Society of London.Series A, Mathematical and Physical Sciences, 333 (1973) 297-316.

23. R. Taylor, R. Krishna, Multicomponent mass transfer, Wiley, New York, 1993.

24. C.N. Satterfield, C.K. Colton, W.H. Pitcher, Restricted diffusion in liquids within fine pores, AIChE J., 19 (1973) 628-635.

25. H. Brenner, L.J. Gaydos, The constrained brownian movement of spherical particles in cylindrical pores of comparable radius. Models of the diffusive and convective transport of solute molecules in membranes and porous media, J.Colloid Interface Sci., 58 (1977) 312-356.

26. F. Gritti, G. Guiochon, Mass transfer kinetics, band broadening and column efficiency, Journal of Chromatography A, 1221 (2012) 2-40.

27. B. Coquebert de Neuville, A. Tarafder, M. Morbidelli, Distributed pore model for bio-molecule chromatography, Journal of Chromatography A, 1298 (2013) 26-34.

28. J. van Brakel, P.M. Heertjes, Analysis of diffusion in macroporous media in terms of a porosity, a tortuosity and a constrictivity factor, International Journal of Heat and Mass Transfer, 17 (1974) 10931103.

29. C.N. Satterfield, Mass transfer in heterogeneous catalysis, M.I.T. Press, 1970.

30. M. Suzuki, Adsorption engineering, Kodansha, 1990.

31. K. Miyabe, G. Guiochon, Measurement of the parameters of the mass transfer kinetics in high performance liquid chromatography, Journal of Separation Science, 26 (2003) 155-173.

32. B. Coquebert de Neuville, H. Thomas, M. Morbidelli, Simulation of porosity decrease with protein adsorption using the distributed pore model, Journal of Chromatography A, 1314 (2013) 77-85.

33. Y. Yao, K.J. Czymmek, R. Pazhianur, A.M. Lenhoff, Three-Dimensional Pore Structure of Chromatographic Adsorbents from Electron Tomography, Langmuir, 22 (2006) 11148-11157.

34. G. Carta, A. Jungbauer, Adsorption Kinetics, Protein Chromatography, Wiley-VCH Verlag GmbH \& Co. KGaA, 2010, pp. 161-199.

35. F. Gritti, G. Guiochon, General HETP Equation for the Study of Mass-Transfer Mechanisms in RPLC, Anal.Chem., 78 (2006) 5329-5347.

36. E.M. Renkin, Filtration, Diffusion, and Molecular Sieving Through Porous Cellulose Memranes, J.Gen.Physiol., 38 (1954) 225-243.

37. F. Gritti, G. Guiochon, Effect of the surface coverage of C18-bonded silica particles on the obstructive factor and intraparticle diffusion mechanism, Chemical engineering science., 61 (2006) 7636. 
38. S. Khirevich, A. Höltzel, A. Daneyko, A. Seidel-Morgenstern, U. Tallarek, Structure-transport correlation for the diffusive tortuosity of bulk, monodisperse, random sphere packings, Journal of Chromatography A, 1218 (2011) 6489-6497.

39. N. Epstein, On tortuosity and the tortuosity factor in flow and diffusion through porous media, in: Chemical Engineering Science, 1989, pp. 777-779.

40. J.C. Giddings, Dynamics of Chromatography: Principles and Theory, CRC Press, 2017.

41. S. Chalk, L. McEwen, The IUPAC Gold Book, Chemistry International, 39 (2017).

42. F. Gritti, G. Guiochon, Application of the general height equivalent to a theoretical plate equation to size exclusion chromatography. Study of the mass transfer of high-molecular-mass compounds in liquid chromatography, Anal.Chem., 79 (2007) 3188-3198.

43. J.J. Meyers, A.I. Liapis, Network modeling of the intraparticle convection and diffusion of molecules in porous particles packed in a chromatographic column, Journal of Chromatography A, 827 (1998) 197-213.

44. J.C. Giddings, Dynamics of Chromatography. Part. I: Principles and Theory, Marcel Dekker, New York, 1965.

45. D. Wiedenmann, L. Keller, L. Holzer, J. Stojadinović, B. Münch, L. Suarez, B. Fumey, H. Hagendorfer, R. Brönnimann, P. Modregger, M. Gorbar, U.F. Vogt, A. Züttel, F.L. Mantia, R. Wepf, B. Grobéty, Three-dimensional pore structure and ion conductivity of porous ceramic diaphragms, AIChE J., 59 (2013) 1446-1457.

46. M. Barrande, R. Bouchet, R. Denoyel, Tortuosity of porous particles, Anal.Chem., 79 (2007) 91159121.

47. P. Aggarwal, V. Asthana, J.S. Lawson, H.D. Tolley, D.R. Wheeler, B.A. Mazzeo, M.L. Lee, Correlation of chromatographic performance with morphological features of organic polymer monoliths, Journal of Chromatography A, 1334 (2014) 20-29.

48. K. Diao, L. Zhang, Y. Zhao, Measurement of tortuosity of porous $\mathrm{Cu}$ using a diffusion diaphragm cell, Measurement, 110 (2017) 335-338.

49. J. Landesfeind, J. Hattendorff, A. Ehrl, W.A. Wall, H.A. Gasteiger, Tortuosity Determination of Battery Electrodes and Separators by Impedance Spectroscopy, J.Electrochem.Soc., 163 (2016) A1373-A1387.

50. M. Matyka, A. Khalili, Z. Koza, Tortuosity-porosity relation in porous media flow, Physical Review E - Statistical, Nonlinear, and Soft Matter Physics, 78 (2008) 1-8.

51. I.V. Thorat, D.E. Stephenson, N.A. Zacharias, K. Zaghib, J.N. Harb, D.R. Wheeler, Quantifying tortuosity in porous Li-ion battery materials, J.Power Sources, 188 (2009) 592-600.

52. L. Chen, L. Chen, X. Yan, Q.-H. Wan, Measurement of packing tortuosity and porosity in capillary electrochromatography, Anal.Chem., 74 (2002) 5157-5159.

53. G.M. Laudone, C.M. Gribble, K.L. Jones, H.J. Collier, G.P. Matthews, Validated a priori calculation of tortuosity in porous materials including sandstone and limestone, Chemical Engineering Science, 131 (2015) 109-117.

54. N.O. Shanti, V.W.L. Chan, S.R. Stock, F. De Carlo, K. Thornton, K.T. Faber, X-ray micro-computed tomography and tortuosity calculations of percolating pore networks, Acta Materialia, 71 (2014) 126135 .

55. I.V. Thorat, D.E. Stephenson, N.A. Zacharias, K. Zaghib, J.N. Harb, D.R. Wheeler, Quantifying tortuosity in porous Li-ion battery materials, in: J.Power Sources, 2009, pp. 592-600. 
56. B. Tjaden, D.P. Finegan, J. Lane, D.J.L. Brett, P.R. Shearing, Contradictory concepts in tortuosity determination in porous media in electrochemical devices, in: Chemical Engineering Science, 2017, pp. 235-245.

57. Y. Yao, K.J. Czymmek, R. Pazhianur, A.M. Lenhoff, Three-dimensional pore structure of chromatographic adsorbents from electron tomography, Langmuir : the ACS journal of surfaces and colloids, 22 (2006) 11148-11157.

58. J.J. Meyers, S. Nahar, D.K. Ludlow, A.I. Liapis, Determination of the pore connectivity and pore size distribution and pore spatial distribution of porous chromatographic particles from nitrogen sorption measurements and pore network modelling theory, (2000).

59. J.F. Langford, M.R. Schure, Y. Yao, S.F. Maloney, A.M. Lenhoff, Effects of pore structure and molecular size on diffusion in chromatographic adsorbents, The Role of Theory in Chromatography, 1126 (2006) 95-106.

60. L. Hagel, Chapter 5 Pore Size Distributions, Aqueous Size-Exclusion Chromatography, Elsevier, 1988, pp. 119-155.

61. S. Ergun, Fluid flow through packed columns, Chemical Engineering Progress, 48 (1952).

62. A. Schultze-Jena, M.A. Boon, P.J.T. Bussmann, A.E.M. Janssen, A. van der Padt, The counterintuitive role of extra-column volume in the determination of column efficiency and scaling of chromatographic processes, Journal of Chromatography A, 1493 (2017) 49-56.

63. J.J. Baeza-Baeza, S. Pous-Torres, J.R. Torres-Lapasió, M.C. García-Álvarez-Coque, Approaches to characterise chromatographic column performance based on global parameters accounting for peak broadening and skewness, Journal of Chromatography A, 1217 (2010) 2147-2157.

64. A.J. Alexander, T.J. Waeghe, K.W. Himes, F.P. Tomasella, T.F. Hooker, Modifying conventional high-performance liquid chromatography systems to achieve fast separations with Fused-Core columns: A case study, Journal of Chromatography A, 1218 (2011) 5456-5469.

65. F. Gritti, A. Felinger, G. Guiochon, Influence of the errors made in the measurement of the extracolumn volume on the accuracies of estimates of the column efficiency and the mass transfer kinetics parameters, Journal of Chromatography A, 1136 (2006) 57-72.

66. D.S. Eisenberg, W. Kauzmann, The structure and properties of water, Clarendon Press (New York) ; Oxford University Press (Oxford), 2005.

67. R.C. Reid, J.M. Prausnitz, B.E. Poling, The properties of gases and liquids, (1987).

68. E.J. Wilson, C.J. Geankoplis, Liquid Mass Transfer at Very Low Reynolds Numbers in Packed Beds, Industrial \& Engineering Chemistry Fundamentals, 5 (1966) 9-14.

69. P. DePhillips, A.M. Lenhoff, Pore size distributions of cation-exchange adsorbents determined by inverse size-exclusion chromatography, Journal of Chromatography A, 883 (2000) 39-54.

70. L. Hagel, M. Östberg, T. Andersson, Apparent pore size distributions of chromatography media, in: Journal of Chromatography A, 1996, pp. 33-42.

71. A.L. Zydney, P. Aimar, M. Meireles, J.M. Pimbley, G. Belfort, Use of the log-normal probability density function to analyze membrane pore size distributions: functional forms and discrepancies, J.Membr.Sci., 91 (1994) 293-298.

72. G. Healthcare, Column efficiency testing, in, https://www.gelifesciences.com, 2010.

73. J.C. Williams, L.A. Mark, S. Eichholtz, Partition and Permeation of Dextran in Polyacrylamide Gel, in: Biophys.J., 1998, pp. 493-502. 
74. J. Gutenwik, B. Nilsson, A. Axelsson, Effect of hindered diffusion on the adsorption of proteins in agarose gel using a pore model, Journal of Chromatography A, 1048 (2004) 161-172.

75. T. Bioscience, Toyopearl® Instruction Manual, in, https://www.separations.eu.tosohbioscience.com, 2015. 


\subsection{Supplementary material}

\subsubsection{Van Deemter curves}
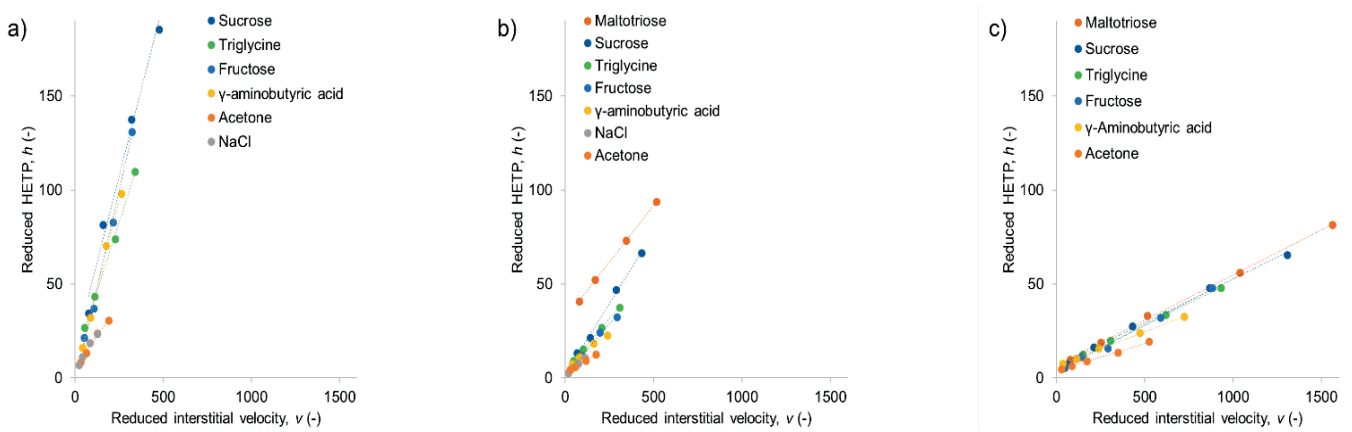

Fig. 3.8 - Van Deemter curves in a) Sephadex G-10, b) G-15, and c) G-25. Reduced HETP $h$ (-) over reduced velocity $v(-)$.
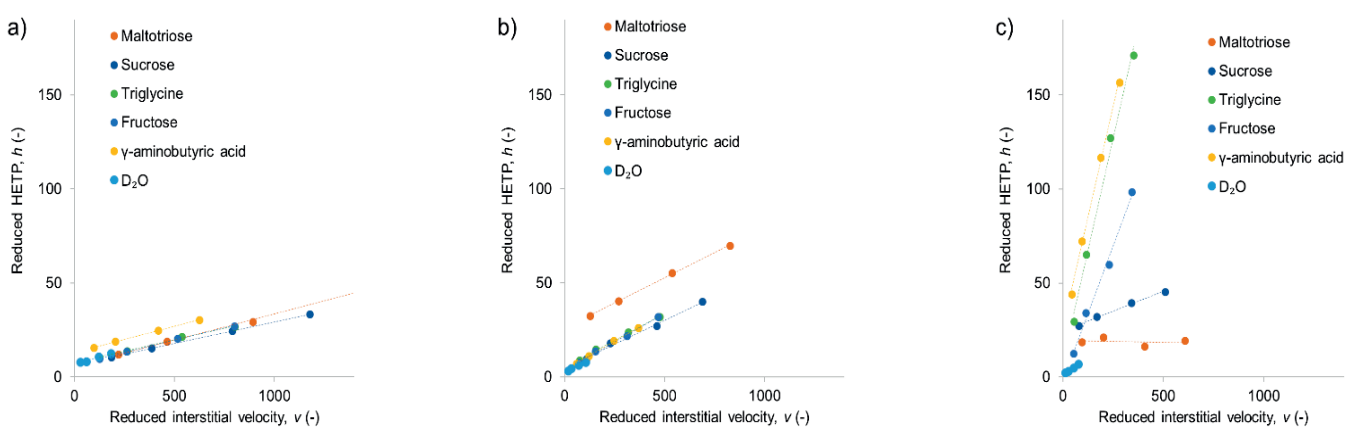

Fig. 3.9 - Van Deemter curves in a) Dowex 50XW2, b) XW4 and c) XW8. Reduced HETP $h$ (-) over reduced velocity $v(-)$.
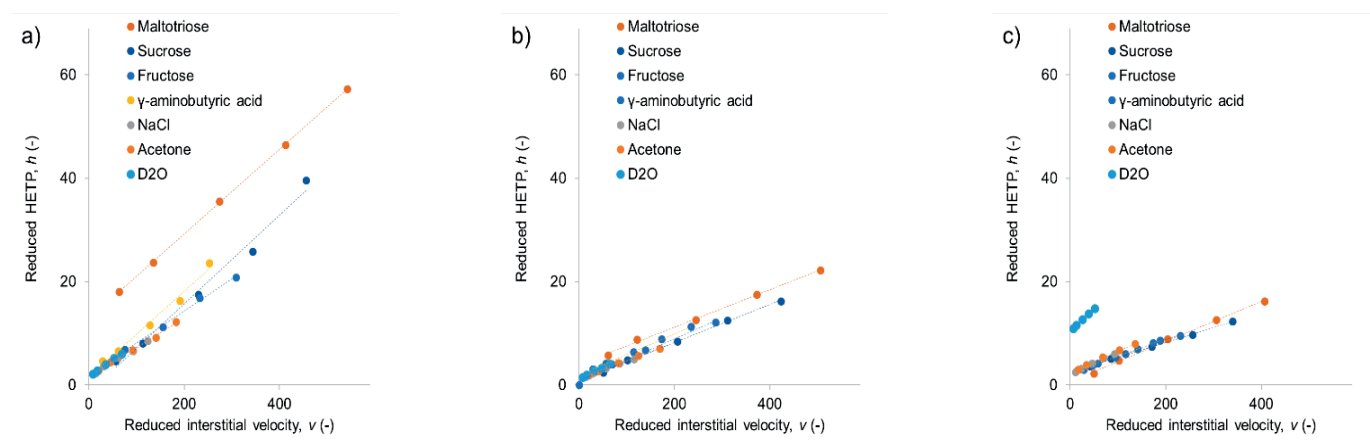

Fig. 3.10 - Van Deemter curves in a) Toyopearl HW40F, b) 50F, and c) 65F. Reduced HETP $h(-)$ over reduced velocity $v(-)$. 


\subsubsection{Column data}

Table 3.5 - Data of each column packed with a different stationary phase. All columns with an inner diameter of $10 \mathrm{~mm}$.

\begin{tabular}{r|rrrrrrrr}
\multicolumn{1}{c}{ Stationary phase } & $\boldsymbol{L}(\boldsymbol{m})$ & $\boldsymbol{r}_{\boldsymbol{p}}(\boldsymbol{\mu m})$ & $\boldsymbol{V}_{\boldsymbol{0}}(\boldsymbol{m} \boldsymbol{L})$ & $\boldsymbol{V}_{\boldsymbol{t}}(\boldsymbol{m} \boldsymbol{L})$ & $\boldsymbol{\varepsilon b}(-)$ & $\boldsymbol{\varepsilon t}(-)$ & $\boldsymbol{\varepsilon}_{p}(-)$ & $\boldsymbol{r}_{\text {pore }}(\boldsymbol{n m})$ \\
\hline Sephadex G-10 & 0.30 & 43.84 & 8.56 & 15.35 & 0.37 & 0.66 & 0.46 & 1.0 \\
Sephadex G-15 & 0.26 & 37.07 & 7.12 & 16.05 & 0.34 & 0.78 & 0.66 & 1.4 \\
Sephadex G-25 & 0.24 & 131.19 & 7.67 & 15.93 & 0.40 & 0.84 & 0.73 & 1.7 \\
Dowex 50WX2 & 0.21 & 70.39 & 3.92 & 14.61 & 0.24 & 0.88 & 0.84 & 2.3 \\
Dowex 50WX4 & 0.21 & 52.85 & 5.00 & 12.82 & 0.30 & 0.78 & 0.68 & 1.4 \\
Dowex 50WX8 & 0.22 & 45.47 & 6.18 & 11.97 & 0.36 & 0.69 & 0.52 & 0.7 \\
Toyopearl HW40F & 0.21 & 24.22 & 4.67 & 12.36 & 0.28 & 0.75 & 0.66 & 1.7 \\
Toyopearl HW50F & 0.24 & 24.78 & 6.04 & 15.28 & 0.32 & 0.81 & 0.72 & 5.0 \\
Toyopearl HW65F & 0.21 & 26.05 & 7.05 & 13.63 & 0.42 & 0.81 & 0.68 & 35.0
\end{tabular}




\subsubsection{Elution data}

Table 3.6 - Elution data of all molecules in Sephadex G-10 stationary phase. Elution of $\mathrm{D}_{2} \mathrm{O}$ for different velocities was not measured in this stationary phase.

\begin{tabular}{|c|c|c|c|c|c|}
\hline Molecule & $u_{S}(m / h)$ & $u_{L}(m / s)$ & $\mu_{I}(m L)$ & $\mu_{2}\left(m L^{2}\right)$ & $\operatorname{HETP}(m)$ \\
\hline \multirow{4}{*}{$\mathrm{D}_{2} \mathrm{O}$} & - & - & - & - & - \\
\hline & - & - & - & - & - \\
\hline & - & - & - & - & - \\
\hline & - & - & - & - & - \\
\hline \multirow{4}{*}{ Acetone } & 0.49 & 0.0004 & 14.84 & 0.56 & 0.0008 \\
\hline & 1.00 & 0.0007 & 15.07 & 0.89 & 0.0012 \\
\hline & 2.02 & 0.0015 & 15.22 & 1.63 & 0.0021 \\
\hline & 3.03 & 0.0022 & 15.34 & 2.16 & 0.0027 \\
\hline \multirow{4}{*}{$\mathrm{NaCl}$} & 0.48 & 0.0003 & 13.53 & 0.38 & 0.0006 \\
\hline & 1.00 & 0.0007 & 14.01 & 0.66 & 0.0010 \\
\hline & 2.03 & 0.0015 & 14.28 & 1.13 & 0.0016 \\
\hline & 3.03 & 0.0022 & 14.31 & 1.43 & 0.0021 \\
\hline \multirow{4}{*}{$\gamma$-aminobutyric acid } & 0.48 & 0.0004 & 11.00 & 0.58 & 0.0014 \\
\hline & 1.00 & 0.0007 & 11.41 & 1.24 & 0.0028 \\
\hline & 2.03 & 0.0015 & 11.42 & 2.73 & 0.0062 \\
\hline & 3.03 & 0.0022 & 11.46 & 3.85 & 0.0086 \\
\hline \multirow{4}{*}{ Fructose } & 0.48 & 0.0004 & 12.21 & 0.95 & 0.0019 \\
\hline & 1.00 & 0.0007 & 12.66 & 1.77 & 0.0032 \\
\hline & 2.03 & 0.0015 & 12.95 & 4.14 & 0.0073 \\
\hline & 3.03 & 0.0022 & 13.19 & 6.80 & 0.0115 \\
\hline \multirow{4}{*}{ Triglycine } & 0.48 & 0.0004 & 10.45 & 0.87 & 0.0024 \\
\hline & 1.00 & 0.0007 & 10.76 & 1.49 & 0.0038 \\
\hline & 2.03 & 0.0015 & 10.88 & 2.61 & 0.0065 \\
\hline & 3.03 & 0.0022 & 10.93 & 3.91 & 0.0096 \\
\hline \multirow{4}{*}{ Sucrose } & 0.48 & 0.0004 & 10.96 & 1.23 & 0.0030 \\
\hline & 1.00 & 0.0007 & 11.31 & 3.11 & 0.0072 \\
\hline & 2.03 & 0.0015 & 11.23 & 5.16 & 0.0121 \\
\hline & 3.03 & 0.0022 & 11.19 & 6.92 & 0.0163 \\
\hline \multirow{4}{*}{ Maltotriose } & 0.48 & 0.0004 & 10.02 & 2.09 & 0.0061 \\
\hline & 1.00 & 0.0007 & 9.97 & 1.99 & 0.0059 \\
\hline & 2.03 & 0.0015 & 9.52 & 1.39 & 0.0045 \\
\hline & 3.03 & 0.0022 & 9.36 & 1.02 & 0.0034 \\
\hline
\end{tabular}


Table 3.7 - Elution data of all molecules in Sephadex $G$ - 15 stationary phase. Elution of $\mathrm{D}_{2} \mathrm{O}$ for different velocities was not measured in this stationary phase.

\begin{tabular}{|c|c|c|c|c|c|}
\hline Molecule & $u_{s}(m / h)$ & $u_{L}(\mathrm{~m} / \mathrm{s})$ & $\mu_{1}(m L)$ & $\mu_{2}\left(m L^{2}\right)$ & $\operatorname{HETP}(m)$ \\
\hline \multirow{4}{*}{$\mathrm{D}_{2} \mathrm{O}$} & - & - & - & - & - \\
\hline & - & - & - & - & . \\
\hline & - & - & - & - & - \\
\hline & - & - & - & - & - \\
\hline \multirow{4}{*}{ Acetone } & 0.47 & 0.0004 & 15.57 & 0.27 & 0.0003 \\
\hline & 1.00 & 0.0008 & 16.42 & 0.41 & 0.0004 \\
\hline & 2.03 & 0.0016 & 16.56 & 0.65 & 0.0007 \\
\hline & 3.03 & 0.0024 & 16.63 & 0.88 & 0.0009 \\
\hline \multirow{4}{*}{$\mathrm{NaCl}$} & 0.47 & 0.0004 & 14.96 & 0.16 & 0.0002 \\
\hline & 1.00 & 0.0008 & 15.80 & 0.33 & 0.0004 \\
\hline & 2.03 & 0.0015 & 16.00 & 0.50 & 0.0006 \\
\hline & 3.02 & 0.0023 & 16.06 & 0.71 & 0.0008 \\
\hline \multirow{4}{*}{$\gamma$-aminobutyric acid } & 0.50 & 0.0004 & 13.03 & 0.32 & 0.0005 \\
\hline & 1.00 & 0.0008 & 13.04 & 0.48 & 0.0008 \\
\hline & 2.03 & 0.0016 & 13.20 & 0.81 & 0.0014 \\
\hline & 3.02 & 0.0024 & 13.24 & 1.02 & 0.0017 \\
\hline \multirow{4}{*}{ Fructose } & 0.47 & 0.0004 & 13.80 & 0.31 & 0.0005 \\
\hline & 1.00 & 0.0008 & 14.58 & 0.63 & 0.0009 \\
\hline & 2.03 & 0.0016 & 14.72 & 1.34 & 0.0018 \\
\hline & 3.03 & 0.0024 & 14.83 & 1.82 & 0.0024 \\
\hline \multirow{4}{*}{ Triglycine } & 0.47 & 0.0004 & 11.67 & 0.32 & 0.0007 \\
\hline & 1.00 & 0.0008 & 12.29 & 0.59 & 0.0011 \\
\hline & 2.03 & 0.0016 & 12.46 & 1.06 & 0.0020 \\
\hline & 3.03 & 0.0024 & 12.52 & 1.49 & 0.0028 \\
\hline \multirow{4}{*}{ Sucrose } & 0.47 & 0.0004 & 12.58 & 0.54 & 0.0010 \\
\hline & 1.00 & 0.0008 & 13.19 & 0.95 & 0.0016 \\
\hline & 2.02 & 0.0016 & 13.24 & 2.09 & 0.0035 \\
\hline & 3.03 & 0.0024 & 13.42 & 3.05 & 0.0049 \\
\hline \multirow{4}{*}{ Maltotriose } & 0.47 & 0.0004 & 11.43 & 1.36 & 0.0030 \\
\hline & 0.99 & 0.0008 & 11.77 & 1.85 & 0.0039 \\
\hline & 2.03 & 0.0016 & 11.81 & 2.60 & 0.0054 \\
\hline & 3.03 & 0.0024 & 11.81 & 3.33 & 0.0069 \\
\hline
\end{tabular}


Table 3.8 - Elution data of all molecules in Sephadex G-25 stationary phase. Elution of $\mathrm{D}_{2} \mathrm{O}$ for different velocities was not measured in this stationary phase.

\begin{tabular}{|c|c|c|c|c|c|}
\hline Molecule & $u_{S}(m / h)$ & $u_{L}(m / s)$ & $\mu_{I}(m L)$ & $\mu_{2}\left(m L^{2}\right)$ & $\operatorname{HETP}(m)$ \\
\hline \multirow{5}{*}{$D_{2} \mathrm{O}$} & . & - & - & - & - \\
\hline & - & - & - & - & - \\
\hline & - & - & - & - & - \\
\hline & - & - & - & - & - \\
\hline & - & - & - & - & - \\
\hline \multirow{5}{*}{ Acetone } & 0.16 & 0.0001 & 16.20 & 1.22 & 0.0012 \\
\hline & 0.50 & 0.0003 & 16.48 & 1.74 & 0.0017 \\
\hline & 1.00 & 0.0007 & 16.32 & 2.39 & 0.0023 \\
\hline & 2.02 & 0.0014 & 16.59 & 3.74 & 0.0036 \\
\hline & 3.04 & 0.0021 & 16.75 & 5.45 & 0.0051 \\
\hline \multirow{5}{*}{$\mathrm{NaCl}$} & - & - & - & - & - \\
\hline & - & - & - & - & - \\
\hline & - & - & - & - & - \\
\hline & - & - & - & - & - \\
\hline & - & - & - & - & - \\
\hline \multirow{5}{*}{$\gamma$-aminobutyric acid } & 0.15 & 0.0001 & 14.09 & 1.54 & 0.0020 \\
\hline & 0.49 & 0.0003 & 15.55 & 2.44 & 0.0027 \\
\hline & 1.00 & 0.0007 & 15.72 & 3.94 & 0.0042 \\
\hline & 2.02 & 0.0014 & 15.99 & 6.06 & 0.0063 \\
\hline & 3.04 & 0.0021 & 15.88 & 8.29 & 0.0086 \\
\hline \multirow{5}{*}{ Fructose } & 0.15 & 0.0001 & 14.96 & 1.26 & 0.0015 \\
\hline & 0.49 & 0.0003 & 15.95 & 2.82 & 0.0030 \\
\hline & 1.00 & 0.0007 & 16.18 & 4.12 & 0.0042 \\
\hline & 2.02 & 0.0014 & 16.42 & 8.56 & 0.0084 \\
\hline & 3.03 & 0.0021 & 16.63 & 13.10 & 0.0126 \\
\hline \multirow{5}{*}{ Triglycine } & 0.15 & 0.0001 & 14.32 & 1.09 & 0.0014 \\
\hline & 0.49 & 0.0003 & 15.17 & 2.87 & 0.0033 \\
\hline & 1.00 & 0.0007 & 15.29 & 4.68 & 0.0052 \\
\hline & 2.02 & 0.0014 & 15.60 & 8.13 & 0.0089 \\
\hline & 3.04 & 0.0021 & 15.58 & 11.72 & 0.0126 \\
\hline \multirow{5}{*}{ Sucrose } & 0.15 & 0.0001 & 14.09 & 1.48 & 0.0020 \\
\hline & 0.49 & 0.0003 & 14.95 & 3.58 & 0.0043 \\
\hline & 1.00 & 0.0007 & 15.09 & 6.28 & 0.0072 \\
\hline & 2.02 & 0.0014 & 15.34 & 11.17 & 0.0126 \\
\hline & 3.04 & 0.0021 & 15.27 & 15.31 & 0.0172 \\
\hline \multirow{5}{*}{ Maltotriose } & 0.15 & 0.0001 & 13.03 & 1.65 & 0.0025 \\
\hline & 0.49 & 0.0003 & 13.73 & 3.58 & 0.0050 \\
\hline & 1.00 & 0.0007 & 13.96 & 6.49 & 0.0087 \\
\hline & 2.02 & 0.0014 & 13.94 & 10.81 & 0.0147 \\
\hline & 3.04 & 0.0021 & 14.05 & 16.16 & 0.0214 \\
\hline
\end{tabular}


Table 3.9 - Elution data of all molecules in Dowex 50WX2 stationary phase. Elution of acetone and $\mathrm{NaCl}$ for different velocities were not measured in this stationary phase.

\begin{tabular}{|c|c|c|c|c|c|}
\hline Molecule & $u_{S}(m / h)$ & $u_{L}(m / s)$ & $\mu_{I}(m L)$ & $\mu_{2}\left(m L^{2}\right)$ & $\operatorname{HETP}(m)$ \\
\hline \multirow{4}{*}{$\mathrm{D}_{2} \mathrm{O}$} & 0.49 & 0.0006 & 14.30 & 1.10 & 0.0011 \\
\hline & 0.99 & 0.0011 & 14.41 & 1.15 & 0.0012 \\
\hline & 2.03 & 0.0023 & 14.89 & 1.59 & 0.0015 \\
\hline & 3.06 & 0.0035 & 14.85 & 1.83 & 0.0018 \\
\hline \multirow{4}{*}{ Acetone } & - & - & - & - & - \\
\hline & - & - & - & - & - \\
\hline & - & - & - & - & - \\
\hline & - & - & - & - & - \\
\hline \multirow{4}{*}{$\mathrm{NaCl}$} & - & - & - & - & - \\
\hline & - & - & - & - & - \\
\hline & - & - & - & - & - \\
\hline & - & - & - & - & - \\
\hline \multirow{4}{*}{$\gamma$-aminobutyric acid } & 0.47 & 0.0005 & 14.69 & 2.23 & 0.0022 \\
\hline & 0.99 & 0.0011 & 15.40 & 2.99 & 0.0027 \\
\hline & 2.02 & 0.0022 & 15.71 & 4.07 & 0.0035 \\
\hline & 3.02 & 0.0033 & 15.88 & 5.09 & 0.0043 \\
\hline \multirow{4}{*}{ Fructose } & 0.47 & 0.0005 & 12.49 & 1.00 & 0.0014 \\
\hline & 0.99 & 0.0011 & 13.16 & 1.57 & 0.0019 \\
\hline & 1.95 & 0.0022 & 12.85 & 2.24 & 0.0029 \\
\hline & 3.02 & 0.0035 & 13.73 & 3.40 & 0.0038 \\
\hline \multirow{4}{*}{ Triglycine } & 0.47 & 0.0005 & 18.78 & 2.47 & 0.0015 \\
\hline & 0.99 & 0.0011 & 19.71 & 3.59 & 0.0020 \\
\hline & 2.02 & 0.0022 & 20.19 & 5.79 & 0.0030 \\
\hline & 3.01 & 0.0033 & 20.18 & 7.27 & 0.0038 \\
\hline \multirow{4}{*}{ Sucrose } & 0.47 & 0.0005 & 10.55 & 0.78 & 0.0015 \\
\hline & 0.99 & 0.0011 & 11.11 & 1.27 & 0.0022 \\
\hline & 2.02 & 0.0023 & 11.41 & 2.11 & 0.0034 \\
\hline & 3.01 & 0.0035 & 11.47 & 2.92 & 0.0047 \\
\hline \multirow{4}{*}{ Maltotriose } & 0.47 & 0.0005 & 10.34 & 0.86 & 0.0017 \\
\hline & 0.99 & 0.0011 & 11.14 & 1.57 & 0.0027 \\
\hline & 1.91 & 0.0022 & 11.06 & 2.41 & 0.0041 \\
\hline & 3.02 & 0.0035 & 11.85 & 4.26 & 0.0064 \\
\hline
\end{tabular}


Table 3.10 - Elution data of all molecules in Dowex 50WX4 stationary phase. Elution of acetone and $\mathrm{NaCl}$ for different velocities were not measured in this stationary phase.

\begin{tabular}{|c|c|c|c|c|c|}
\hline Molecule & $u_{S}(m / h)$ & $u_{L}(m / s)$ & $\mu_{I}(m L)$ & $\mu_{2}\left(m L^{2}\right)$ & $\operatorname{HETP}(m)$ \\
\hline \multirow{4}{*}{$\mathrm{D}_{2} \mathrm{O}$} & 0.48 & 0.0004 & 12.54 & 0.26 & 0.0003 \\
\hline & 0.96 & 0.0009 & 12.53 & 0.36 & 0.0005 \\
\hline & 2.03 & 0.0018 & 13.19 & 0.55 & 0.0007 \\
\hline & 3.03 & 0.0027 & 13.01 & 0.66 & 0.0008 \\
\hline \multirow{4}{*}{ Acetone } & - & - & - & - & - \\
\hline & - & - & - & - & - \\
\hline & - & - & - & - & - \\
\hline & - & - & - & - & - \\
\hline \multirow{4}{*}{$\mathrm{NaCl}$} & - & - & - & - & - \\
\hline & - & - & - & - & - \\
\hline & - & - & - & - & - \\
\hline & - & - & - & - & - \\
\hline \multirow{4}{*}{$\gamma$-aminobutyric acid } & 0.48 & 0.0004 & 12.08 & 0.53 & 0.0008 \\
\hline & 0.99 & 0.0009 & 12.35 & 0.86 & 0.0012 \\
\hline & 2.02 & 0.0017 & 12.63 & 1.56 & 0.0020 \\
\hline & 3.03 & 0.0026 & 12.72 & 2.13 & 0.0028 \\
\hline \multirow{4}{*}{ Fructose } & 0.47 & 0.0004 & 10.05 & 0.39 & 0.0008 \\
\hline & 0.99 & 0.0009 & 10.71 & 0.79 & 0.0014 \\
\hline & 2.02 & 0.0018 & 10.83 & 1.30 & 0.0023 \\
\hline & 3.03 & 0.0027 & 11.31 & 2.06 & 0.0034 \\
\hline \multirow{4}{*}{ Triglycine } & 0.48 & 0.0004 & 15.75 & 1.12 & 0.0009 \\
\hline & 0.99 & 0.0009 & 16.12 & 1.93 & 0.0016 \\
\hline & 2.03 & 0.0018 & 16.66 & 3.33 & 0.0025 \\
\hline & 3.03 & 0.0026 & 16.61 & 4.46 & 0.0034 \\
\hline \multirow{4}{*}{ Sucrose } & 0.48 & 0.0004 & 8.01 & 0.31 & 0.0010 \\
\hline & 1.00 & 0.0009 & 8.34 & 0.63 & 0.0019 \\
\hline & 2.02 & 0.0018 & 8.38 & 0.97 & 0.0029 \\
\hline & 3.02 & 0.0027 & 8.41 & 1.43 & 0.0042 \\
\hline \multirow{4}{*}{ Maltotriose } & 0.47 & 0.0004 & 7.67 & 0.97 & 0.0034 \\
\hline & 0.99 & 0.0009 & 8.20 & 1.38 & 0.0043 \\
\hline & 1.97 & 0.0018 & 8.23 & 1.91 & 0.0058 \\
\hline & 3.03 & 0.0027 & 8.26 & 2.41 & 0.0074 \\
\hline
\end{tabular}


Table 3.11 - Elution data of all molecules in Dowex 50WX8 stationary phase. Elution of acetone and $\mathrm{NaCl}$ for different velocities were not measured in this stationary phase.

\begin{tabular}{|c|c|c|c|c|c|}
\hline Molecule & $u_{S}(m / h)$ & $u_{L}(\mathrm{~m} / \mathrm{s})$ & $\mu_{I}(m L)$ & $\mu_{2}\left(m L^{2}\right)$ & $\operatorname{HETP}(m)$ \\
\hline \multirow{4}{*}{$\mathrm{D}_{2} \mathrm{O}$} & 0.47 & 0.0004 & 11.43 & 0.13 & 0.0002 \\
\hline & 1.00 & 0.0008 & 12.21 & 0.19 & 0.0003 \\
\hline & 2.02 & 0.0016 & 12.22 & 0.31 & 0.0005 \\
\hline & 3.02 & 0.0023 & 12.04 & 0.42 & 0.0006 \\
\hline \multirow{4}{*}{ Acetone } & - & - & - & - & - \\
\hline & - & - & - & - & - \\
\hline & - & - & - & - & - \\
\hline & - & - & - & - & - \\
\hline \multirow{4}{*}{$\mathrm{NaCl}$} & - & - & - & - & - \\
\hline & - & - & - & - & - \\
\hline & - & - & - & - & - \\
\hline & - & - & - & - & - \\
\hline \multirow{4}{*}{$\gamma$-aminobutyric acid } & 0.47 & 0.0004 & 9.95 & 1.79 & 0.0040 \\
\hline & 1.00 & 0.0008 & 10.72 & 3.41 & 0.0066 \\
\hline & 2.02 & 0.0016 & 10.46 & 5.26 & 0.0106 \\
\hline & 3.02 & 0.0023 & 10.48 & 7.08 & 0.0143 \\
\hline \multirow{4}{*}{ Fructose } & 0.47 & 0.0004 & 8.27 & 0.35 & 0.0011 \\
\hline & 1.00 & 0.0008 & 8.98 & 1.13 & 0.0031 \\
\hline & 2.02 & 0.0016 & 8.81 & 1.91 & 0.0054 \\
\hline & 3.02 & 0.0023 & 9.19 & 3.42 & 0.0090 \\
\hline \multirow{4}{*}{ Triglycine } & 0.47 & 0.0004 & 10.99 & 1.47 & 0.0027 \\
\hline & 1.00 & 0.0007 & 11.79 & 3.72 & 0.0059 \\
\hline & 2.02 & 0.0015 & 11.83 & 7.32 & 0.0116 \\
\hline & 3.02 & 0.0023 & 11.75 & 9.71 & 0.0156 \\
\hline \multirow{4}{*}{ Sucrose } & 0.47 & 0.0004 & 6.52 & 0.47 & 0.0025 \\
\hline & 1.00 & 0.0008 & 6.83 & 0.61 & 0.0029 \\
\hline & 2.02 & 0.0016 & 6.66 & 0.72 & 0.0036 \\
\hline & 3.02 & 0.0023 & 6.72 & 0.84 & 0.0041 \\
\hline \multirow{4}{*}{ Maltotriose } & 0.47 & 0.0004 & 5.99 & 0.28 & 0.0017 \\
\hline & 1.00 & 0.0008 & 6.40 & 0.35 & 0.0019 \\
\hline & 2.02 & 0.0016 & 6.42 & 0.28 & 0.0015 \\
\hline & 3.02 & 0.0023 & 6.32 & 0.32 & 0.0018 \\
\hline
\end{tabular}


Table 3.12 - Elution data of all molecules in Toyopearl HW-4OF stationary phase. Elution of triglycine for different velocities was not measured in this stationary phase.

\begin{tabular}{|c|c|c|c|c|c|}
\hline Molecule & $u_{S}(m / h)$ & $u_{L}(m / s)$ & $\mu_{I}(m L)$ & $\mu_{2}\left(m L^{2}\right)$ & $\operatorname{HETP}(m)$ \\
\hline \multirow{5}{*}{$\mathrm{D}_{2} \mathrm{O}$} & 0.50 & 0.0005 & 12.44 & 0.08 & 0.0001 \\
\hline & 1.00 & 0.0010 & 12.30 & 0.10 & 0.0001 \\
\hline & 2.03 & 0.0020 & 12.50 & 0.15 & 0.0002 \\
\hline & 3.04 & 0.0029 & 12.42 & 0.19 & 0.0003 \\
\hline & 4.03 & 0.0039 & 12.17 & 0.21 & 0.0003 \\
\hline \multirow{5}{*}{ Acetone } & 0.46 & 0.0004 & 12.31 & 0.10 & 0.0001 \\
\hline & 0.99 & 0.0010 & 13.18 & 0.18 & 0.0002 \\
\hline & 2.03 & 0.0020 & 13.58 & 0.29 & 0.0003 \\
\hline & 3.10 & 0.0030 & 13.82 & 0.41 & 0.0004 \\
\hline & 4.05 & 0.0039 & 13.38 & 0.51 & 0.0006 \\
\hline \multirow{5}{*}{$\mathrm{NaCl}$} & 0.50 & 0.0005 & 11.25 & 0.06 & 0.0001 \\
\hline & 0.99 & 0.0010 & 10.39 & 0.09 & 0.0002 \\
\hline & 2.02 & 0.0020 & 11.49 & 0.14 & 0.0003 \\
\hline & 3.04 & 0.0029 & 10.88 & 0.18 & 0.0003 \\
\hline & 4.03 & 0.0039 & 10.60 & 0.22 & 0.0004 \\
\hline \multirow{5}{*}{$\gamma$-aminobutyric acid } & 0.46 & 0.0004 & 7.90 & 0.07 & 0.0002 \\
\hline & 0.99 & 0.0010 & 8.61 & 0.11 & 0.0003 \\
\hline & 2.04 & 0.0020 & 8.94 & 0.21 & 0.0006 \\
\hline & 3.05 & 0.0030 & 8.84 & 0.30 & 0.0008 \\
\hline & 4.04 & 0.0039 & 8.63 & 0.41 & 0.0011 \\
\hline \multirow{5}{*}{ Fructose } & 0.46 & 0.0004 & 8.81 & 0.07 & 0.0002 \\
\hline & 0.99 & 0.0010 & 9.64 & 0.15 & 0.0003 \\
\hline & 2.03 & 0.0020 & 9.82 & 0.25 & 0.0005 \\
\hline & 3.04 & 0.0029 & 10.01 & 0.39 & 0.0008 \\
\hline & 4.03 & 0.0039 & 9.58 & 0.44 & 0.0010 \\
\hline \multirow{5}{*}{ Triglycine } & - & - & - & - & - \\
\hline & - & - & - & - & - \\
\hline & - & - & - & - & - \\
\hline & - & - & - & - & - \\
\hline & - & - & - & - & - \\
\hline \multirow{5}{*}{ Sucrose } & 0.50 & 0.0005 & 8.89 & 0.08 & 0.0002 \\
\hline & 1.00 & 0.0010 & 8.92 & 0.15 & 0.0004 \\
\hline & 2.03 & 0.0020 & 9.11 & 0.34 & 0.0008 \\
\hline & 3.04 & 0.0029 & 9.11 & 0.50 & 0.0013 \\
\hline & 4.03 & 0.0039 & 8.73 & 0.70 & 0.0019 \\
\hline \multirow{5}{*}{ Maltotriose } & 0.47 & 0.0005 & 7.86 & 0.26 & 0.0009 \\
\hline & 1.00 & 0.0010 & 8.12 & 0.36 & 0.0011 \\
\hline & 2.03 & 0.0020 & 8.34 & 0.57 & 0.0017 \\
\hline & 3.05 & 0.0030 & 8.16 & 0.71 & 0.0022 \\
\hline & 4.01 & 0.0039 & 8.04 & 0.86 & 0.0028 \\
\hline
\end{tabular}


Table 3.13 - Elution data of all molecules in Toyopearl HW-50F stationary phase. Elution of acetone and $\mathrm{NaCl}$ for different velocities were not measured in this stationary phase. Fructose only at 4 different velocities.

\begin{tabular}{|c|c|c|c|c|c|}
\hline Molecule & $u_{S}(m / h)$ & $u_{L}(m / s)$ & $\mu_{1}(m L)$ & $\mu_{2}\left(m L^{2}\right)$ & $\operatorname{HETP}(m)$ \\
\hline \multirow{5}{*}{$\mathrm{D}_{2} \mathrm{O}$} & 0.50 & 0.0004 & 15.28 & 0.07 & 0.0001 \\
\hline & 1.00 & 0.0009 & 15.43 & 0.10 & 0.0001 \\
\hline & 2.01 & 0.0017 & 15.45 & 0.14 & 0.0001 \\
\hline & 3.04 & 0.0026 & 15.59 & 0.16 & 0.0002 \\
\hline & 4.13 & 0.0035 & 15.84 & 0.21 & 0.0002 \\
\hline \multirow{5}{*}{ Acetone } & 0.50 & 0.0004 & 16.16 & 0.10 & 0.0001 \\
\hline & 0.99 & 0.0009 & 16.11 & 0.14 & 0.0001 \\
\hline & 2.01 & 0.0017 & 16.38 & 0.24 & 0.0002 \\
\hline & 3.03 & 0.0026 & 16.41 & 0.32 & 0.0003 \\
\hline & 4.13 & 0.0035 & 16.72 & 0.41 & 0.0004 \\
\hline \multirow{5}{*}{$\mathrm{NaCl}$} & 0.50 & 0.0004 & 14.07 & 0.06 & 0.0001 \\
\hline & 1.00 & 0.0009 & 13.92 & 0.09 & 0.0001 \\
\hline & 2.01 & 0.0017 & 14.09 & 0.13 & 0.0002 \\
\hline & 3.04 & 0.0026 & 14.21 & 0.17 & 0.0002 \\
\hline & 4.13 & 0.0035 & 14.60 & 0.22 & 0.0002 \\
\hline \multirow{5}{*}{$\gamma$-aminobutyric acid } & 0.50 & 0.0004 & 12.47 & 0.09 & 0.0002 \\
\hline & 1.00 & 0.0009 & 12.53 & 0.12 & 0.0002 \\
\hline & 2.01 & 0.0017 & 12.59 & 0.23 & 0.0003 \\
\hline & 3.05 & 0.0026 & 12.67 & 0.29 & 0.0004 \\
\hline & 4.13 & 0.0035 & 12.90 & 0.39 & 0.0006 \\
\hline \multirow{5}{*}{ Fructose } & 0.50 & 0.0004 & 13.51 & 0.10 & 0.0001 \\
\hline & 1.00 & 0.0009 & 13.36 & 0.15 & 0.0002 \\
\hline & 2.01 & 0.0017 & 13.47 & 0.25 & 0.0003 \\
\hline & - & - & - & - & - \\
\hline & 4.13 & 0.0035 & 13.65 & 0.46 & 0.0006 \\
\hline \multirow{5}{*}{ Triglycine } & - & - & - & - & - \\
\hline & - & - & - & - & - \\
\hline & - & - & - & - & - \\
\hline & - & - & - & - & - \\
\hline & - & - & - & - & - \\
\hline \multirow{5}{*}{ Sucrose } & 0.50 & 0.0004 & 12.69 & 0.08 & 0.0001 \\
\hline & 0.99 & 0.0008 & 12.64 & 0.16 & 0.0002 \\
\hline & 2.01 & 0.0017 & 12.80 & 0.29 & 0.0004 \\
\hline & 3.04 & 0.0026 & 13.00 & 0.43 & 0.0006 \\
\hline & 4.13 & 0.0035 & 13.05 & 0.57 & 0.0008 \\
\hline \multirow{5}{*}{ Maltotriose } & 0.50 & 0.0004 & 12.27 & 0.18 & 0.0003 \\
\hline & 0.99 & 0.0008 & 11.99 & 0.26 & 0.0004 \\
\hline & 2.00 & 0.0017 & 11.99 & 0.37 & 0.0006 \\
\hline & 3.04 & 0.0026 & 12.16 & 0.53 & 0.0009 \\
\hline & 4.13 & 0.0035 & 12.38 & 0.70 & 0.0011 \\
\hline
\end{tabular}


Table 3.14 - Elution data of all molecules in Toyopearl HW-65F stationary phase. Elution of acetone and $\mathrm{NaCl}$ for different velocities were not measured in this stationary phase.

\begin{tabular}{|c|c|c|c|c|c|}
\hline Molecule & $u_{S}(m / h)$ & $u_{L}(m / s)$ & $\mu_{1}(m L)$ & $\mu_{2}\left(m L^{2}\right)$ & $\operatorname{HETP}(m)$ \\
\hline \multirow{5}{*}{$\mathrm{D}_{2} \mathrm{O}$} & 0.49 & 0.0003 & 13.32 & 0.48 & 0.0006 \\
\hline & 1.00 & 0.0007 & 13.72 & 0.53 & 0.0006 \\
\hline & 2.02 & 0.0014 & 13.79 & 0.59 & 0.0007 \\
\hline & 3.03 & 0.0020 & 13.66 & 0.63 & 0.0007 \\
\hline & 4.03 & 0.0027 & 13.65 & 0.68 & 0.0008 \\
\hline \multirow{5}{*}{ Acetone } & 0.49 & 0.0003 & 14.47 & 0.15 & 0.0002 \\
\hline & 1.00 & 0.0007 & 14.83 & 0.21 & 0.0002 \\
\hline & 2.02 & 0.0014 & 15.00 & 0.30 & 0.0003 \\
\hline & 3.03 & 0.0020 & 14.88 & 0.36 & 0.0004 \\
\hline & 4.03 & 0.0027 & 14.84 & 0.43 & 0.0004 \\
\hline \multirow{5}{*}{$\mathrm{NaCl}$} & 0.49 & 0.0003 & 13.20 & 0.11 & 0.0001 \\
\hline & 1.00 & 0.0007 & 13.54 & 0.14 & 0.0002 \\
\hline & 2.02 & 0.0014 & 13.83 & 0.19 & 0.0002 \\
\hline & 3.03 & 0.0020 & 13.78 & 0.24 & 0.0003 \\
\hline & 4.03 & 0.0027 & 13.80 & 0.28 & 0.0003 \\
\hline \multirow{5}{*}{$\gamma$-aminobutyric acid } & 0.49 & 0.0003 & 12.53 & 0.12 & 0.0002 \\
\hline & 1.01 & 0.0007 & 12.94 & 0.16 & 0.0002 \\
\hline & 2.02 & 0.0014 & 12.97 & 0.22 & 0.0003 \\
\hline & 3.03 & 0.0020 & 12.84 & 0.28 & 0.0004 \\
\hline & 4.03 & 0.0027 & 12.84 & 0.35 & 0.0004 \\
\hline \multirow{5}{*}{ Fructose } & 0.49 & 0.0003 & 12.81 & 0.12 & 0.0002 \\
\hline & 1.01 & 0.0007 & 13.24 & 0.18 & 0.0002 \\
\hline & 2.02 & 0.0014 & 13.26 & 0.27 & 0.0003 \\
\hline & 3.03 & 0.0020 & 13.39 & 0.36 & 0.0004 \\
\hline & 4.03 & 0.0027 & 13.08 & 0.40 & 0.0005 \\
\hline \multirow{5}{*}{ Triglycine } & - & - & - & - & - \\
\hline & - & - & - & - & - \\
\hline & - & - & - & - & - \\
\hline & - & - & - & - & - \\
\hline & - & - & - & - & - \\
\hline \multirow{5}{*}{ Sucrose } & 0.49 & 0.0003 & 12.58 & 0.14 & 0.0002 \\
\hline & 1.01 & 0.0007 & 12.95 & 0.21 & 0.0003 \\
\hline & 2.02 & 0.0014 & 13.02 & 0.31 & 0.0004 \\
\hline & 3.03 & 0.0020 & 12.97 & 0.40 & 0.0005 \\
\hline & 4.03 & 0.0027 & 12.63 & 0.48 & 0.0006 \\
\hline \multirow{5}{*}{ Maltotriose } & 0.49 & 0.0003 & 12.13 & 0.08 & 0.0001 \\
\hline & 1.01 & 0.0007 & 12.76 & 0.19 & 0.0002 \\
\hline & 2.02 & 0.0014 & 12.91 & 0.37 & 0.0005 \\
\hline & 3.03 & 0.0020 & 12.67 & 0.49 & 0.0007 \\
\hline & 4.03 & 0.0027 & 12.72 & 0.64 & 0.0008 \\
\hline
\end{tabular}




\subsubsection{Inverted size exclusion data}

Table 3.15 - Mean retention volume $V_{R}$, void volume $V_{0}$, total liquid volume $V_{T}$, partition coefficient $K_{D}$, and molecular radius $r_{m}$ for the Sephadex series used for the $K_{D}$ curves to calculate pore size distribution.

\begin{tabular}{|c|c|c|c|c|c|c|}
\hline Stationary phase & Molecule & $V_{R}\left(\mu_{I}\right)[m L]$ & $V_{0}[m L]$ & $V_{T}[m L]$ & $K_{D}[-]$ & $r_{m}[n m]$ \\
\hline \multirow[t]{7}{*}{ Sephadex G-10 } & Dextran $2 \cdot 10^{6}$ & 8.18 & 8.18 & 15.95 & 0.000 & 36.706 \\
\hline & $\mathrm{D}_{2} \mathrm{O}$ & 15.95 & 8.18 & 15.95 & 1.000 & 0.094 \\
\hline & Fructose & 11.82 & 8.18 & 15.95 & 0.468 & 0.323 \\
\hline & Sucrose & 10.39 & 8.18 & 15.95 & 0.284 & 0.478 \\
\hline & Maltotriose & 9.46 & 8.18 & 15.95 & 0.165 & 0.601 \\
\hline & $\gamma$-aminobutyric acid & 10.57 & 8.18 & 15.95 & 0.308 & 0.272 \\
\hline & Triglycine & 10.02 & 8.18 & 15.95 & 0.237 & 0.369 \\
\hline \multirow[t]{9}{*}{ Sephadex G-15 } & Dextran $2 \cdot 10^{6}$ & 7.56 & 7.56 & 16.87 & 0.000 & 36.706 \\
\hline & $\mathrm{D}_{2} \mathrm{O}$ & 16.87 & 7.56 & 16.87 & 1.000 & 0.094 \\
\hline & Fructose & 13.29 & 7.56 & 16.87 & 0.615 & 0.323 \\
\hline & Sucrose & 12.11 & 7.56 & 16.87 & 0.488 & 0.478 \\
\hline & Maltotriose & 11.06 & 7.56 & 16.87 & 0.376 & 0.601 \\
\hline & Maltotetraose & 9.81 & 7.56 & 16.87 & 0.241 & 0.691 \\
\hline & Dextran $1 K$ & 8.70 & 7.56 & 16.87 & 0.122 & 0.886 \\
\hline & $\gamma$-aminobutyric acid & 11.24 & 7.56 & 16.87 & 0.395 & 0.272 \\
\hline & Triglycine & 11.97 & 7.56 & 16.87 & 0.474 & 0.369 \\
\hline \multirow[t]{10}{*}{ Sephadex $G-25$} & Dextran $2 \cdot 10^{6}$ & 7.97 & 7.97 & 17.90 & 0.000 & 36.706 \\
\hline & $\mathrm{D}_{2} \mathrm{O}$ & 17.90 & 7.97 & 17.90 & 1.000 & 0.094 \\
\hline & Fructose & 16.32 & 7.97 & 17.90 & 0.840 & 0.323 \\
\hline & Sucrose & 15.11 & 7.97 & 17.90 & 0.719 & 0.571 \\
\hline & Maltotriose & 13.63 & 7.97 & 17.90 & 0.570 & 0.601 \\
\hline & Maltotetraose & 11.08 & 7.97 & 17.90 & 0.313 & 0.691 \\
\hline & Dextran $1 K$ & 10.14 & 7.97 & 17.90 & 0.218 & 0.886 \\
\hline & Dextran $5 K$ & 7.83 & 7.97 & 17.90 & -0.014 & 1.768 \\
\hline & $\gamma$-aminobutyric acid & 15.84 & 7.97 & 17.90 & 0.792 & 0.272 \\
\hline & Triglycine & 15.44 & 7.97 & 17.90 & 0.752 & 0.369 \\
\hline
\end{tabular}


Table 3.16 - Mean retention volume $V_{R}$, void volume $V_{0}$, total liquid volume $V_{T}$, partition coefficient $K_{D}$, and molecular radius $r_{m}$ for the Dowex series used for the $K_{D}$ curves to calculate pore size distribution.

\begin{tabular}{|c|c|c|c|c|c|c|}
\hline Stationary phase & Molecule & $V_{R}\left(\mu_{I}\right)[m L]$ & $V_{0}[m L]$ & $V_{T}[m L]$ & $K_{D}[-]$ & $r_{m}[n m]$ \\
\hline \multirow[t]{10}{*}{ Dowex $50 W X 2$} & Dextran $2 \cdot 10^{6}$ & 5.86 & 5.86 & 18.49 & 0.000 & 36.706 \\
\hline & $\mathrm{D}_{2} \mathrm{O}$ & 18.49 & 5.86 & 18.49 & 1.000 & 0.094 \\
\hline & Fructose & 16.86 & 5.86 & 18.49 & 0.871 & 0.323 \\
\hline & Sucrose & 14.40 & 5.86 & 18.49 & 0.676 & 0.571 \\
\hline & Maltotriose & 14.18 & 5.86 & 18.49 & 0.659 & 0.601 \\
\hline & Maltotetraose & 14.13 & 5.86 & 18.49 & 0.655 & 0.691 \\
\hline & $\operatorname{Dex} 1 K$ & 10.95 & 5.86 & 18.49 & 0.403 & 0.886 \\
\hline & $\operatorname{Dex} 5 K$ & 6.40 & 5.86 & 18.49 & 0.042 & 1.768 \\
\hline & $\gamma$-aminobutyric acid & 19.46 & 5.86 & 18.49 & 1.077 & 0.272 \\
\hline & Triglycine & 24.79 & 5.86 & 18.49 & 1.499 & 0.369 \\
\hline \multirow[t]{7}{*}{ Dowex 50WX4 } & Dextran $2 \cdot 10^{6}$ & 7.37 & 7.37 & 17.81 & 0.000 & 36.706 \\
\hline & $\mathrm{D}_{2} \mathrm{O}$ & 17.81 & 7.37 & 17.81 & 1.000 & 0.094 \\
\hline & Fructose & 14.76 & 7.37 & 17.81 & 0.708 & 0.323 \\
\hline & Sucrose & 11.45 & 7.37 & 17.81 & 0.391 & 0.571 \\
\hline & Maltotriose & 11.10 & 7.37 & 17.81 & 0.358 & 0.601 \\
\hline & Maltotetraose & 10.21 & 7.37 & 17.81 & 0.272 & 0.691 \\
\hline & $\operatorname{Dex} 1 K$ & 8.69 & 7.37 & 17.81 & 0.127 & 0.886 \\
\hline \multirow[t]{7}{*}{ Dowex 50WX8 } & Dextran $2 \cdot 10^{6}$ & 7.96 & 7.96 & 15.92 & 0.000 & 36.706 \\
\hline & $\mathrm{D}_{2} \mathrm{O}$ & 15.92 & 7.96 & 15.92 & 1.000 & 0.094 \\
\hline & Fructose & 11.50 & 7.96 & 15.92 & 0.445 & 0.323 \\
\hline & Sucrose & 8.96 & 7.96 & 15.92 & 0.125 & 0.571 \\
\hline & Maltotriose & 8.41 & 7.96 & 15.92 & 0.055 & 0.601 \\
\hline & Maltotetraose & 8.04 & 7.96 & 15.92 & 0.010 & 0.691 \\
\hline & $\operatorname{DexlK}$ & 8.01 & 7.96 & 15.92 & 0.006 & 0.886 \\
\hline
\end{tabular}


Table 3.17 - Mean retention volume $V_{R}$, void volume $V_{0}$, total liquid volume $V_{T}$, partition coefficient $K_{D}$, and molecular radius $r_{m}$ for the Toyopearl series used for the $K_{D}$ curves to calculate pore size distribution.

\begin{tabular}{|c|c|c|c|c|c|c|}
\hline Stationary phase & Molecule & $V_{R}\left(\mu_{I}\right)[m L]$ & $V_{0}[m L]$ & $V_{T}[m L]$ & $K_{D}[-]$ & $r_{m}[n m]$ \\
\hline \multirow[t]{10}{*}{ Toyopearl HW 40-F } & Dextran $2 \cdot 10^{6}$ & 4.01 & 4.01 & 12.97 & 0.000 & 36.706 \\
\hline & $\mathrm{D}_{2} \mathrm{O}$ & 12.97 & 4.01 & 12.97 & 1.000 & 0.094 \\
\hline & Fructose & 9.72 & 4.01 & 12.97 & 0.638 & 0.323 \\
\hline & Sucrose & 8.87 & 4.01 & 12.97 & 0.542 & 0.571 \\
\hline & Maltotriose & 7.89 & 4.01 & 12.97 & 0.433 & 0.601 \\
\hline & Maltotetraose & 6.98 & 4.01 & 12.97 & 0.331 & 0.691 \\
\hline & Dex $1 K$ & 6.02 & 4.01 & 12.97 & 0.224 & 0.886 \\
\hline & $\operatorname{Dex} 5 K$ & 4.29 & 4.01 & 12.97 & 0.031 & 1.768 \\
\hline & $\gamma$-aminobutyric acid & 8.50 & 4.01 & 12.97 & 0.501 & 0.272 \\
\hline & Triglycine & 7.75 & 4.01 & 12.97 & 0.418 & 0.369 \\
\hline \multirow[t]{13}{*}{ Toyopearl HW 50-F } & Dextran $2 \cdot 10^{6}$ & 5.41 & 5.41 & 15.42 & 0.000 & 36.706 \\
\hline & $\mathrm{D}_{2} \mathrm{O}$ & 15.42 & 5.41 & 15.42 & 1.000 & 0.094 \\
\hline & Fructose & 13.07 & 5.41 & 15.42 & 0.765 & 0.323 \\
\hline & Sucrose & 12.45 & 5.41 & 15.42 & 0.704 & 0.571 \\
\hline & Maltotriose & 11.78 & 5.41 & 15.42 & 0.636 & 0.601 \\
\hline & Maltotetraose & 11.13 & 5.41 & 15.42 & 0.571 & 0.691 \\
\hline & Dex $1 K$ & 10.43 & 5.41 & 15.42 & 0.501 & 0.886 \\
\hline & Dex $5 k$ & 8.58 & 5.41 & 15.42 & 0.317 & 1.768 \\
\hline & Dex $12 K$ & 7.67 & 5.41 & 15.42 & 0.226 & 2.661 \\
\hline & Dex $25 K$ & 6.78 & 5.41 & 15.42 & 0.137 & 3.757 \\
\hline & Dex $50 k$ & 6.09 & 5.41 & 15.42 & 0.069 & 5.627 \\
\hline & $\gamma$-aminobutyric acid & 12.37 & 5.41 & 15.42 & 0.695 & 0.272 \\
\hline & Triglycine & 11.87 & 5.41 & 15.42 & 0.646 & 0.369 \\
\hline \multirow[t]{16}{*}{ Toyopearl HW 65-F } & Dextran $2 \cdot 10^{6}$ & 9.18 & 9.18 & 18.05 & 0.000 & 36.706 \\
\hline & $\mathrm{D}_{2} \mathrm{O}$ & 19.33 & 9.18 & 18.05 & 1.145 & 0.094 \\
\hline & Fructose & 18.05 & 9.18 & 19.33 & 0.873 & 0.323 \\
\hline & Sucrose & 17.48 & 9.18 & 19.33 & 0.818 & 0.571 \\
\hline & Maltotriose & 17.29 & 9.18 & 19.33 & 0.799 & 0.601 \\
\hline & Maltotetraose & 17.12 & 9.18 & 19.33 & 0.782 & 0.691 \\
\hline & $\operatorname{DexlK}$ & 17.00 & 9.18 & 19.33 & 0.770 & 0.89 \\
\hline & $\operatorname{Dex} 5 K$ & 16.41 & 9.18 & 19.33 & 0.712 & 1.77 \\
\hline & Dex $12 K$ & 16.23 & 9.18 & 19.33 & 0.695 & 2.66 \\
\hline & Dex $25 K$ & 15.66 & 9.18 & 19.33 & 0.638 & 3.76 \\
\hline & Dex $50 K$ & 15.14 & 9.18 & 19.33 & 0.587 & 5.63 \\
\hline & Dex $80 K$ & 14.68 & 9.18 & 19.33 & 0.541 & 6.76 \\
\hline & Dex $150 K$ & 14.02 & 9.18 & 19.33 & 0.477 & 9.36 \\
\hline & Dex $270 K$ & 13.46 & 9.18 & 19.33 & 0.422 & 11.68 \\
\hline & Dex $410 K$ & 13.03 & 9.18 & 19.33 & 0.379 & 13.86 \\
\hline & Dex $670 K$ & 12.60 & 9.18 & 19.33 & 0.337 & 16.70 \\
\hline
\end{tabular}




\subsubsection{The parallel pore model - input parameters}

Table 3.18 - Intraparticle diffusivities $D_{p} / D_{m}$, errors of intraparticle diffusivities, hindrance diffusion factors $F\left(\lambda_{m}\right)$, obstruction factors $\gamma_{p}$, partition coefficient $K_{D}$ calculated from the pore size distribution for all molecules in the Sephadex series

\begin{tabular}{|c|c|c|c|c|c|c|}
\hline & Molecule & $D_{p} / D_{m}[-]$ & $\begin{array}{c}\text { Error of } \\
D_{p} / D_{m}[-]\end{array}$ & $F\left(\lambda_{m}\right)[-]$ & $\gamma_{p}[-]$ & $\begin{array}{c}K_{D} \text { from } \\
\text { PoSD [-] }\end{array}$ \\
\hline \multirow[t]{8}{*}{ Sephadex G-10 } & $\mathrm{D}_{2} \mathrm{O}$ & - & - & - & - & - \\
\hline & Acetone & 0.03 & 0.01 & 0.38 & 0.18 & 0.68 \\
\hline & $\mathrm{NaCl}$ & 0.02 & 0.01 & 0.52 & 0.18 & 0.78 \\
\hline & $\gamma$-aminobutyric acid & 0.003 & 0.001 & 0.26 & 0.18 & 0.57 \\
\hline & Fructose & 0.01 & 0.001 & 0.18 & 0.18 & 0.50 \\
\hline & Triglycine & 0.003 & 0.001 & 0.16 & 0.18 & 0.47 \\
\hline & Sucrose & 0.003 & 0.001 & 0.06 & 0.18 & 0.32 \\
\hline & Maltotriose & - & - & - & - & - \\
\hline \multirow[t]{8}{*}{ Sephadex G-15 } & $\mathrm{D}_{2} \mathrm{O}$ & - & - & - & - & - \\
\hline & Acetone & 0.11 & 0.02 & 0.49 & 0.19 & 0.74 \\
\hline & $\mathrm{NaCl}$ & 0.07 & 0.03 & 0.61 & 0.19 & 0.82 \\
\hline & $\gamma$-aminobutyric acid & 0.05 & 0.02 & 0.38 & 0.19 & 0.57 \\
\hline & Fructose & 0.04 & 0.01 & 0.30 & 0.19 & 0.59 \\
\hline & Triglycine & 0.03 & 0.01 & 0.28 & 0.19 & 0.65 \\
\hline & Sucrose & 0.02 & 0.01 & 0.15 & 0.19 & 0.43 \\
\hline & Maltotriose & 0.02 & 0.005 & 0.08 & 0.19 & 0.32 \\
\hline \multirow[t]{8}{*}{ Sephadex G-25 } & $\mathrm{D}_{2} \mathrm{O}$ & - & - & - & - & - \\
\hline & Acetone & 0.27 & 0.07 & 0.56 & 0.32 & 0.79 \\
\hline & $\mathrm{NaCl}$ & - & - & - & - & - \\
\hline & $\gamma$-aminobutyric acid & 0.18 & 0.04 & 0.45 & 0.32 & 0.72 \\
\hline & Fructose & 0.13 & 0.03 & 0.38 & 0.32 & 0.66 \\
\hline & Triglycine & 0.13 & 0.03 & 0.36 & 0.32 & 0.64 \\
\hline & Sucrose & 0.12 & 0.03 & 0.23 & 0.32 & 0.52 \\
\hline & Maltotriose & 0.10 & 0.02 & 0.15 & 0.32 & 0.42 \\
\hline
\end{tabular}


Table 3.19 - Intraparticle diffusivities $D_{p} / D_{m}$, errors of intraparticle diffusivities, hindrance diffusion factors $F\left(\lambda_{m}\right)$, obstruction factors $\gamma_{p}$, partition coefficient $K_{D}$ calculated from the pore size distribution for all molecules in the Dowex series

\begin{tabular}{|c|c|c|c|c|c|c|}
\hline & Molecule & $D_{p} / D_{m}[-]$ & $\begin{array}{c}\text { Error of } \\
D_{p} / D_{m}[-]\end{array}$ & $F\left(\lambda_{m}\right)[-]$ & $\gamma_{p}[-]$ & $\begin{array}{c}K_{D} \text { from } \\
\text { PoSD [-] }\end{array}$ \\
\hline \multirow[t]{8}{*}{ Dowex 50WX2 } & $\mathrm{D}_{2} \mathrm{O}$ & 0.26 & 0.16 & 0.79 & 0.75 & 0.92 \\
\hline & Acetone & - & - & - & - & - \\
\hline & $\mathrm{NaCl}$ & - & - & - & - & - \\
\hline & $\gamma$-aminobutyric acid & 0.22 & 0.05 & 0.55 & 0.75 & 0.78 \\
\hline & Fructose & 0.21 & 0.05 & 0.48 & 0.75 & 0.73 \\
\hline & Triglycine & 0.29 & 0.09 & 0.46 & 0.75 & 0.72 \\
\hline & Sucrose & 0.20 & 0.04 & 0.34 & 0.75 & 0.62 \\
\hline & Maltotriose & 0.16 & 0.04 & 0.25 & 0.75 & 0.53 \\
\hline \multirow[t]{8}{*}{ Dowex 50WX4 } & $\mathrm{D}_{2} \mathrm{O}$ & 0.16 & 0.07 & 0.69 & 0.75 & 0.87 \\
\hline & Acetone & - & - & - & - & - \\
\hline & $\mathrm{NaCl}$ & - & - & - & - & - \\
\hline & $\gamma$-aminobutyric acid & 0.09 & 0.02 & 0.38 & 0.75 & 0.66 \\
\hline & Fructose & 0.07 & 0.02 & 0.30 & 0.75 & 0.59 \\
\hline & Triglycine & 0.13 & 0.04 & 0.28 & 0.75 & 0.57 \\
\hline & Sucrose & 0.05 & 0.02 & 0.16 & 0.75 & 0.43 \\
\hline & Maltotriose & 0.04 & 0.01 & 0.08 & 0.75 & 0.32 \\
\hline \multirow[t]{8}{*}{ Dowex 50WX8 } & $\mathrm{D}_{2} \mathrm{O}$ & 0.08 & 0.02 & 0.48 & 0.36 & 0.73 \\
\hline & Acetone & - & - & - & - & - \\
\hline & $\mathrm{NaCl}$ & - & - & - & - & - \\
\hline & $\gamma$-aminobutyric acid & 0.01 & 0.002 & 0.11 & 0.36 & 0.36 \\
\hline & Fructose & 0.01 & 0.002 & 0.05 & 0.36 & 0.26 \\
\hline & Triglycine & 0.01 & 0.003 & 0.04 & 0.36 & 0.24 \\
\hline & Sucrose & 0.002 & 0.001 & 0.004 & 0.36 & 0.10 \\
\hline & Maltotriose & - & - & - & - & - \\
\hline
\end{tabular}


Table 3.20 - Intraparticle diffusivities $D_{p} / D_{m}$, errors of intraparticle diffusivities, hindrance diffusion factors $F\left(\lambda_{m}\right)$, obstruction factors $\gamma_{p}$, partition coefficient $K_{D}$ calculated from the pore size distribution for all molecules in the Toyopearl series

\begin{tabular}{|c|c|c|c|c|c|c|}
\hline & Molecule & $D_{p} / D_{m}[-]$ & $\begin{array}{c}\text { Error of } \\
D_{p} / D_{m}[-]\end{array}$ & $F\left(\lambda_{m}\right)[-]$ & $\gamma_{p}[-]$ & $\begin{array}{c}K_{D} \text { from } \\
\text { PoSD [-] }\end{array}$ \\
\hline \multirow[t]{8}{*}{ Toyopearl HW-4OF } & $\mathrm{D}_{2} \mathrm{O}$ & 0.11 & 0.03 & 0.74 & 0.24 & 0.89 \\
\hline & Acetone & 0.11 & 0.03 & 0.56 & 0.24 & 0.78 \\
\hline & $\mathrm{NaCl}$ & 0.08 & 0.02 & 0.66 & 0.24 & 0.85 \\
\hline & $\gamma$-aminobutyric acid & 0.03 & 0.01 & 0.45 & 0.24 & 0.70 \\
\hline & Fructose & 0.06 & 0.01 & 0.38 & 0.24 & 0.64 \\
\hline & Triglycine & - & - & - & - & - \\
\hline & Sucrose & 0.04 & 0.01 & 0.23 & 0.24 & 0.50 \\
\hline & Maltotriose & 0.03 & 0.01 & 0.14 & 0.24 & 0.41 \\
\hline \multirow[t]{8}{*}{ Toyopearl HW-50F } & $\mathrm{D}_{2} \mathrm{O}$ & 0.20 & 0.08 & 0.89 & 0.37 & 0.95 \\
\hline & Acetone & 0.20 & 0.05 & 0.80 & 0.37 & 0.91 \\
\hline & $\mathrm{NaCl}$ & 0.17 & 0.05 & 0.85 & 0.37 & 0.94 \\
\hline & $\gamma$-aminobutyric acid & 0.11 & 0.02 & 0.74 & 0.37 & 0.88 \\
\hline & Fructose & 0.13 & 0.04 & 0.70 & 0.37 & 0.85 \\
\hline & Triglycine & - & - & - & - & - \\
\hline & Sucrose & 0.13 & 0.03 & 0.60 & 0.37 & 0.78 \\
\hline & Maltotriose & 0.12 & 0.03 & 0.54 & 0.37 & 0.73 \\
\hline \multirow[t]{8}{*}{ Toyopearl HW-65F } & $\mathrm{D}_{2} \mathrm{O}$ & 0.10 & 0.02 & 0.98 & 0.48 & 0.99 \\
\hline & Acetone & 0.20 & 0.05 & 0.96 & 0.48 & 0.99 \\
\hline & $\mathrm{NaCl}$ & 0.15 & 0.04 & 0.97 & 0.48 & 0.99 \\
\hline & $\gamma$-aminobutyric acid & 0.17 & 0.04 & 0.95 & 0.48 & 0.98 \\
\hline & Fructose & 0.19 & 0.05 & 0.94 & 0.48 & 0.98 \\
\hline & Triglycine & - & - & - & - & - \\
\hline & Sucrose & 0.20 & 0.05 & 0.91 & 0.48 & 0.97 \\
\hline & Maltotriose & 0.13 & 0.03 & 0.89 & 0.48 & 0.96 \\
\hline
\end{tabular}




\section{Chapter 4}

\section{High viscosity preparative chromatography for food applications}

This chapter has been submitted as:

A. Schultze-Jena, M.A. Boon, R.C. Vroon, P.J.Th. Bussmann, A.E.M. Janssen, A. van der Padt.

High viscosity preparative chromatography for food applications 


\section{Abstract}

The strength of chromatography lies in the ability of fine-tuning recovery for specific target components or fractions of interest. A downside of industrial chromatography is the need to dilute streams, as it is often applied today. This article challenges the conventional low concentration of input streams and investigates size exclusion chromatography at concentrated streams of high viscosity. Chromatographic operation with concentrated streams leads to an increased pressure drop over the column and decreased mass transfer kinetics, but also lower volumes compared to diluted streams. The objective of this research was to investigate separation performance and system dimensions as a function of viscosity for food type streams, in scenarios where viscosity is not caused by target components. Disadvantages due to increased stream volume with decreasing concentration and benefits due to decreased viscosity were evaluated, aiming to find minimal column volume.

Separation performance was evaluated for a range of target components in a preparative lab-scale system using a size exclusion resin and mobile phase viscosities in the range of $1.2-8.7 \mathrm{mPa} \cdot \mathrm{s}$. Mobile phases were viscosified through addition of sucrose, glycerol, or dextran. Change in mass transfer resistance, measured via van Deemter curves, was related to the change in diffusivity through viscosity.

The analysis of different viscosifying agents emphasized the influence of viscosity inside the pores, rather than viscosity of the bulk phase. The viscosity inside the pores was calculated via the partition coefficient of each viscosifying agent. Based on the slopes of van Deemter curves, column dimensions were calculated for different scenarios, assuming a non-compressible stationary phase. Column volume remained constant with stream dilution from $8.7 \mathrm{mPa} \cdot \mathrm{s}$ down to about $2.5 \mathrm{mPa} \cdot \mathrm{s}$. However, at the same time column geometry changed to thinner and longer columns with decreasing viscosity, in order to accommodate throughput and pressure drop. When diluting to even lower viscosities, column volume increased, since stream viscosity is less sensitive to stream concentration at the low viscosity range. These results are relevant to a wide range of industries utilizing weak interaction chromatography, especially those where process development is mainly driven by cost reduction and where a trade-off between purity, yield, and costs has to be made. 


\subsection{Introduction}

Preparative chromatographic separations distinguish themselves from most other separation techniques through the ability to fine-tune separation mechanisms for specific molecular properties of target components. This ability allows access to fractions of interest with low loss of product, high purity and high productivity. In order to economize chromatography in industrial processes, process productivity is optimized in regard to product loss, processing time and unit costs. Productivity, generally defined as ratio of product mass to column volume and processing time, is commonly improved by optimizing product yield and/or processing time. Additional potential for productivity optimization lies within reduction of column volume. Column volume is directly linked to chromatographic resin and eluent quantities required, which in many separation processes are the main contributors to overall chromatographic footprint and processing costs and can be reduced if column volume is reduced [1]. One possibility to reduce column volume is reduction of feed stream volume by operation at higher concentrations. But, with increase in concentration, most streams will also show an increase in viscosity, which in turn will have a negative impact on both mass transfer and pressure drop. Commonly, processes are designed to keep stream viscosity as low as possible in order to maximize diffusion and chromatographic efficiency and minimize retention times [2].

Literature on the effect of mobile phase viscosity in liquid column chromatography is primarily concerned with viscous fingering, a phenomenon which reduces column efficiency through instable interfaces between liquid phases of different viscosities [3, 4]; if all liquid phases have the same viscosity, as in this work, viscous fingering does not occur. The effects of viscosity on mass transfer inside chromatographic columns have been discussed to a limited extent. Colin et al. looked at the change in mobile phase viscosity through temperature [5]. They found the main effect of increased temperature on column efficiency and pressure drop is due to the reduced viscosity, which leads to increased diffusivities of the target components. Nakanishi et al. described the dispersion of $\mathrm{NaCl}$ and glucose in size exclusion chromatography, focusing on diffusion inside a range of Sephadex stationary phases [6]. In one part of their work the mobile phase was viscosified with added glycerol to a viscosity of $2.5 \mathrm{mPa} \cdot \mathrm{s}$ and elution curves of $\mathrm{NaCl}$ were measured. The increase in viscosity resulted in an increase in mass transfer resistance. This study builds on the work done by Nakanishi et al. [6]. The mobile phase viscosity was increased even further, up to $8.7 \mathrm{mPa} \cdot \mathrm{s}$.

In general, there are three scenarios where operating at higher stream viscosity may theoretically lead to direct benefits in separation processes. First, inherently viscous streams are diluted before chromatographic processing, only to remove water content of product fractions in subsequent steps. If costly and energy intensive removal of water was minimized by reducing dilution prior to chromatographic separation, process cost savings would be imminent. Second, streams with large volumes and low concentration of target components are often discarded entirely and their product content is not utilized. If the stream volume was reduced, less volume with a higher product concentration could make the purification of target components economically feasible, despite water removal costs. For both scenarios, a chromatographic step under increased viscosity could lead to smaller column volumes and thus processing cost reduction. In a third scenario, chromatography at elevated viscosity may be a viable alternative to chromatography at high temperatures. Many components of complex products are sensitive to temperature and require mild treatment throughout the process. In all three scenarios column length and column diameter will change with input stream viscosity and volume. Relating column volume to bulk viscosity leads to optimized process conditions and minimized water use. 
Evaluation of column volume with changing mobile phase viscosity requires knowledge on column efficiency, stream viscosity, and pressure drop over the column bed, when input stream concentration is changed. Column efficiency is evaluated based on the number of plates $N$, which can be determined as a requirement for a given separation process [5]. The number of plates in a chromatographic column is defined by the ratio of column length $L$ over the height equivalent to a theoretical plate HETP. With the term HETP, fluid dynamic nonidealities, mass transfer resistances, and ad- and desorption rates in a chromatographic column are summed up [5]. One of the most prominent factors on HETP is the diffusion of molecules in mobile and stationary phases, in the latter especially at preparative scale. Molecular diffusivity is directly related to mobile phase properties such as viscosity, temperature, and concentration of its constituents, as well as molecular size or volume of the diffusing molecule itself. Diffusivity in a stationary phase is a more complex matter, but generally speaking it is related to diffusivity in the mobile phase and reduced through (steric) hindrances induced by the porous stationary phase [6]. The influence of concentration on stream viscosity is known for most simple solutions and can be measured in more complex cases. The pressure drop in packed chromatographic beds as a function of viscosity is known. The main unknown is the influence of viscosity on HETP, described by the van Deemter curve.

This study builds on the work done by Colin et al. and Nakanishi et al. [5][6]. The temperature was kept constant and the mobile phase viscosity was increased through addition of viscosifying agents. The effect of viscosity on HETP and system volume was analyzed under the condition that not the target component but the viscosifying agent caused the bulk viscosity. The focus was laid on plate height and the change in number of plates, however the authors are aware that the required number of plates for a separation depends on peak shapes as well. Van Deemter curves, which relate HETP to velocity, were measured at various viscosities. Mobile phase viscosity was increased with three different viscosity agents: sucrose, a common constitute of many food streams such as ketchup, juices and extracts, was compared to glycerol and dextran to analyze influence of molecular characteristics of the viscosifying agent. A new method is demonstrated that enables the comparison of column designs for different stream concentrations based on a constant number of plates and pressure drop. With this method, the influence of viscosity and viscosifier concentration was related to the number of plates $N$ and changes in column dimensions. This research aims at a variety of industries such as food processing or polymer production as well as other processes, where processing costs are limiting factors and, unlike for pharmaceutical requirements, the optimal combination of purity, yield and costs is a trade-off.

\subsection{Theory}

\subsubsection{Height equivalent to a theoretical plate as a function of viscosity}

Preparative chromatography at large scale generally utilizes high velocities to treat large stream volumes and rather large particle diameters to maintain a low pressure drop over the column bed. In such conditions, linear interstitial velocity $u_{L}$ and particle diameter $d_{p}$ are easily a hundred times larger, compared to typical analytical chromatography. When velocity and particle diameter are so large, overall HETP in weak interaction chromatography processes is typically dominated by transparticle mass transfer resistance $H_{\text {Stat }}$ [7], which in 
turn is proportional to the quotient of interstitial linear velocity $u_{L}$ and intraparticle diffusivity $D_{p}$ (equation 4.1).

$$
H E T P \sim H_{\text {Stat }} \propto \frac{u_{L}}{D_{p}}
$$

Intraparticle diffusivity $D_{p}$ is a function of solute interaction with stationary phase properties, here summed up as $\Omega$, and solute diffusivity in the liquid phase inside the pore volume $D_{m \text {.pore }}$ (equation 4.2) [8].

$$
D_{p}=\Omega \cdot D_{\text {m.pore }}
$$

When mobile phase viscosity is influenced by viscosifying agents, solute diffusivity inside pore volume $D_{\text {m.pore }}$ may not be identical to solute diffusivity in the bulk phase $D_{m \text {.bulk }}$. Within the pore volume, molecules are confined by the ratio of their size to pore diameter. This confinement influences the concentration of all molecules in pore volumes and may cause mobile phase viscosity to be different in pore volumes than in the bulk phase. The concentration inside the pores as function of mobile phase concentration can be determined via isotherm measurements. For linear relationships of concentration in bulk and pore, the concentration ratio of each molecule between pore and bulk can be expressed via the partition coefficient $K_{D}$ (equation 4.3) [9].

$$
K_{D}=\frac{c_{\text {pore }}}{c_{\text {bulk }}}
$$

The partition coefficient $K_{D}$ of the viscosity agent can be determined from pulse injection measurements of retention volume of the viscosifying agent $V_{R}$, the interparticle void volume $V_{0}$ and the total mobile phase volume $V_{T}$ (equation 4.4) in phosphate buffer without elevated viscosity [10].

$$
K_{D}=\frac{V_{R}-V_{0}}{V_{T}-V_{0}}
$$

From the concentration of the viscosifying agent in the mobile phase, concentration inside pore volumes is determined and used to calculate an average viscosity $\mu_{p o r e}$ in the pore volume. Solute diffusivity $D_{m . i}\left(\mathrm{~cm}^{2} / \mathrm{s}\right)$ in turn is inversely proportional to viscosity $\mu_{i}$ (either in the bulk or inside the pore), as given by the WilkeChang equation (equation 4.5) [2, 11].

$$
D_{m . i}=\frac{7.4 \cdot 10^{-8}\left(\varphi M_{B . i}\right)^{1 / 2} T}{\mu_{i} V_{A}^{0.6}}
$$

With $\varphi$, the dimensionless association factor of 2.6 for water, $M_{B . i}$ molecular weight of the bulk phase (mixture of phosphate buffer and viscosifier; $\mathrm{g} / \mathrm{mol}), T$ temperature $(\mathrm{K}), \mu_{i}$ viscosity in the bulk or inside the pore volume (mPa.s) and $V_{A}$ molecular volume of solute at boiling point $(\mathrm{mL} / \mathrm{mol})$, calculated after Le Bas [11].

Combining equations 4.1, 4.2, and 4.5, shows that a change of HETP as function of velocity under preparative conditions is proportional to inverse $D_{m \text {.pore }}$, which in turn is inversely proportional to the viscosity inside the pores $\mu_{\text {pore }}$, leading to the relationship of equation 4.6: 


$$
\frac{H E T P}{u_{L}} \propto \frac{1}{D_{\text {m.pore }}} \Rightarrow \frac{\text { HETP }}{u_{L}} \propto \mu_{\text {pore }}
$$

Change in HETP as function of $u_{L}$ is plotted as van Deemter curve and its determination standard chromatographic procedure. Generally van Deemter plots are normalized by dividing HETP by the resin particle diameter $d_{p}$, which yields the reduced HETP $h$ and the linear interstitial velocity $u_{L}$ is multiplied by $d_{p}$ and divided by $D_{m . b u l k}$, which yields the reduced velocity $v$.

\subsubsection{Column dimensions as a function of mobile phase viscosity}

This method enables calculation of column dimensions for different bulk viscosities on the basis of fixed parameters such as a constant pressure drop, required number of plates and initial stream volume.

Column length $L$ and operating velocity $u_{S}$ are calculated for a constant pressure drop $\Delta p$ and a specific viscosity (solid green line in Fig. 4.1). The same parameters $L$ and $u_{S}$ are calculated for a constant number of plates $N$ at that same viscosity (dashed blue line in Fig. 4.1). The intersection of the two lines, marks the operating parameters at which all requirements are met, when utilizing a specific stream viscosity (red circle in Fig. 4.1).

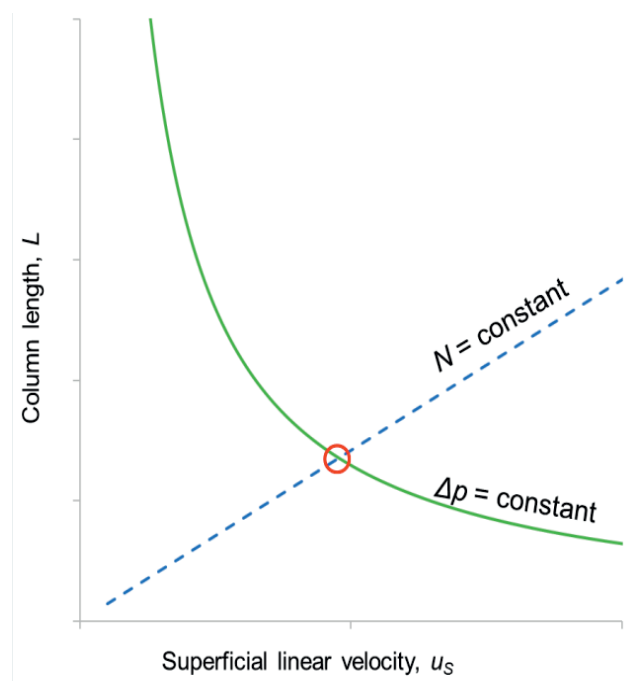

Fig. 4.1 - Column length $L$ and superficial linear velocity $u_{S}$ for set parameters and a specific viscosity. Solid green line shows operation at constant pressure drop over the column $\Delta p$, dashed blue line shows operation at constant number of plates $N$. Intersection of both lines, marked with red circle, gives $L$ and $u_{S}$ at set pressure drop and plate number for a specific viscosity.

Column length for a constant pressure drop over the column bed as function of velocity is calculated with the Ergun equation (equation 4.7), with column length $L(\mathrm{~m})$, pressure drop $\Delta p(\mathrm{~Pa})$, mobile phase viscosity 
$\mu_{b u l k}(\mathrm{~Pa} \cdot \mathrm{s})$, particle diameter $d_{p}(\mathrm{~m})$, bed porosity $\varepsilon_{b}(-)$, mobile phase density $\rho\left(\mathrm{kg} / \mathrm{m}^{3}\right)$, and superficial linear velocity $u_{S}(\mathrm{~m} / \mathrm{s})[12]$.

$$
\frac{1}{L}=\frac{150 \mu_{b u l k}}{d_{p}^{2} \Delta p} \frac{\left(1-\varepsilon_{b}\right)^{2}}{\varepsilon_{b}{ }^{3}} u_{S}+\frac{1.75 \rho}{d_{p} \Delta p} \frac{\left(1-\varepsilon_{b}\right)}{\varepsilon_{b}{ }^{3}} u_{S}^{2}
$$

Equation 4.7 is valid only in case of a non-compressible stationary phase. Many industrially relevant stationary phases do exhibit compression to a certain degree, dependent on operating conditions and column aspect ratios, which must be considered when scaling designs. For such a case Stickel and Fotopoulos derived adjustments to empirical models to correlate pressure drop to aspect ratio of packed beds and velocity and further predict bed porosity [13]. As a first approximation for scale-up, we assumed the stationary phase was non-compressible.

Column length for a constant number of plates as function of linear superficial velocity $u_{S}$ is calculated based on slopes of van Deemter curves and equation 4.8 with $u_{L}=u_{S} / \varepsilon_{b}$.

$$
L=\operatorname{HETP}\left(u_{L}\right) \cdot N
$$

Column volume $C V$ is calculated from $L$ and column area $A$, which in turn was calculated by dividing volumetric stream $Q_{V}$ with linear superficial velocity $u_{S}$. The stream volume $Q_{V}$ is reduced with stream concentration.

With this method the column volume for operation at any given viscosity can be calculated, as long as the van Deemter curve, the pressure drop over the column bed and the viscosifier concentration are known.

\subsection{Materials and methods}

\subsubsection{Materials}

\section{Mobile phase}

All experiments were conducted with a phosphate buffered mobile phase $\left(25 \mathrm{mM} \mathrm{Na}_{2} \mathrm{HPO}_{4}\right.$, $25 \mathrm{mM} \mathrm{NaH}_{2} \mathrm{PO}_{4}$, and $50 \mathrm{mM} \mathrm{NaCl}$; all from Merck, Germany) in Milli-Q water. Viscosity was increased by use of sucrose, a small dextran of 9-11 kDa (both from Sigma Aldrich, St. Louis, MO, USA) or glycerol (Boom, the Netherlands). For the viscous mobile phases, the viscosifier was dissolved first and the salts were added according to the volume of the solution. For each mobile phase the viscosity was measured with a Physica MCR 301 rheometer (Anton Paar, Austria) at $22^{\circ} \mathrm{C}$. Viscosities and concentration of viscosifier added are detailed in Table 4.1 .

Before use mobile phases were filtered through a $0.45 \mu \mathrm{m}$ Durapore ${ }^{\circledR}$ membrane filter (Merck, Germany). Between experiments mobile phases were stored at $4^{\circ} \mathrm{C}$, but no longer than $48 \mathrm{~h}$ before use. 
Table 4.1 - Concentration of sucrose and glycerol $(\% \mathrm{~m} / \mathrm{m})$ to reach target viscosities in each mobile phase.

\begin{tabular}{|c|c|c|c|c|}
\hline Viscosifier \Viscosity & $1.2 \mathrm{mPa} \cdot \mathrm{s}$ & $2.3 \mathrm{mPa} \cdot \mathrm{s}$ & $4.5 \mathrm{mPa} \cdot \mathrm{s}$ & $8.7 \mathrm{mPa} \cdot \mathrm{s}$ \\
\hline Sucrose & $0 \%$ & $22 \%$ & - & $44 \%$ \\
\hline Glycerol & $0 \%$ & - & $46 \%$ & $58 \%$ \\
\hline Dextran & $0 \%$ & - & $19 \%$ & $27.5 \%(8.0 \mathrm{mPa} \cdot \mathrm{s})$ \\
\hline
\end{tabular}

Stationary phase

All experiments were conducted using Sephadex G-15 size exclusion resin (GE Healthcare, Uppsala, Sweden) with a cut off of about $1.5 \mathrm{kDa}$, according to the manufacturers website. The mean particle diameter $(80 \mu \mathrm{m})$ was measured with a Mastersizer 2000 (Malvern, UK).

\section{Target component preparation}

All solid target components were dissolved in the mobile phase at each viscosity, to avoid any changes in viscosity along the column and associated phenomena like viscous fingering. All target components were from Sigma Aldrich, St. Louis, MO, USA. Respective target concentrations and detection wavelengths (refractive index in case of dextran) are given in Table 4.2. Acetone was the only liquid target component and added per volume.

Table 4.2 - Target components, concentration in sample volume and $U V$ detection wavelength ( $R I$ for refractive index).

\begin{tabular}{r|ll} 
Target & $\boldsymbol{c}(\mathbf{g} / \mathbf{L})$ & Detection \\
\hline y-aminobutyric acid & 3 & $210 \mathrm{~nm}$ \\
Triglycerin & 1 & $218 \mathrm{~nm}$ \\
Glycyl-L-tyrosine & 1 & $276 \mathrm{~nm}$ \\
Dextran & 10 & $\mathrm{RI}$ \\
NaCl & 58 & $200 \mathrm{~nm}$ \\
Acetone & $2 \% \mathrm{v} / \mathrm{v}$ & $260 \mathrm{~nm}$
\end{tabular}

\section{Chromatographic equipment}

For liquid chromatography a Wellchrom set-up with a K-1001 pump and a K-2401 RI-detector was used, all from Knauer, Germany. Furthermore a Julabo F25 MP controlled the temperature in the column jacket and a mini Cori-Flow flowmeter (Bronkhorst, The Netherlands) measured the flow rate after the detector. Pressure drop over the column bed was continuously measured using EZG10 pressure sensors (Knauer, Germany), all components were connected with 0.02" PEEK tubing (Grace, Deerfield, IL, USA).

Column measurements were made on a slurry packed Götec Superformance 300-10 column (300 x 10 mm) with tefzel capillaries of $35 \mathrm{~cm}$ lengths and ID $0.5 \mathrm{~mm}$, including flow adapter with frits and filter (all Götec, Germany). During all experiments bed height and pressure drop were recorded and, if required, flow adapters adjusted to minimize headspace upon bed compression or the bed repacked. The zero length column was a Götec Superformance 10-10 column (10 x $10 \mathrm{~mm})$ with no resin and the flow adapters in direct contact as described in [14]. 


\subsubsection{Methods}

\section{Column preparation and characterization}

For experiments at low viscosity, the column was slurry packed in two steps. The first began with phosphate buffer to settle the slurry in a ramped up profile of up to $10 \mathrm{~mL} / \mathrm{min}$ for 20 minutes. In the second step the funnel for the slurry packing was removed, the flow adapter and a filter placed above the resin bed and the resin bed further compressed at $10 \mathrm{~mL} / \mathrm{min}$ for 30 minutes. For measurements at high viscosity, the column was slurry packed in three steps. First, resin slurry was settled in a ramped up flow profile of up to $10 \mathrm{~mL} / \mathrm{min}$ for 20 minutes in phosphate buffer. Second, mobile phase was exchanged to $8.7 \mathrm{mPa} \cdot \mathrm{s}$ sucrose phosphate buffer and flow reduced to $3 \mathrm{~mL} / \mathrm{min}$ for $25 \mathrm{~min}$. Third, the funnel for the slurry packing was removed, the flow adapter and a filter placed above the resin bed and the resin bed compressed at $3 \mathrm{~mL} / \mathrm{min}$ in $8.7 \mathrm{mPa} \cdot \mathrm{s}$ sucrose phosphate buffer for 30 minutes. External porosity was measured via retention volume of $10 \mathrm{~g} / \mathrm{L}$ dextran (average molecular weight of approximately $2 \cdot 10^{6} \mathrm{Da}$ ) pulse injections in phosphate buffer and with blue-dextran (both Sigma Aldrich, St. Louis, MO, USA) at higher viscosities ( $U V$ detection at $621 \mathrm{~nm}$ ).

\section{Chromatographic analysis}

All chromatographic measurements were conducted as pulse injections of $80 \mu \mathrm{L}$ at varying velocity. The column was kept at $22^{\circ} \mathrm{C}$ through a water jacket. All peaks were analyzed with the method of moments in Microsoft Excel as described in [14]. Integration limits were set automatically at $1 \%$ of total peak height and baseline drift was corrected for automatically when necessary to mitigate common concerns of inaccuracy when using the method of moments [15-17]. A detailed example of the correction of drifting baselines and the setting of peak start and end points is given in the supplementary material for $\gamma$-aminobutyric acid in different mobile phases (Fig. 4.12a through 12e). With high viscosifier concentration some target components showed a low signal intensity in their chromatogram, resulting in detection instabilities, noisy baselines and sometimes minima before or after the peak. Among the elution peaks in the supplementary material (Fig. 4.13), $\gamma$-aminobutyric acid eluted in sucrose and glycerol gives a good example of aforementioned detection challenges (Fig. 4.13b). In these cases the integration limits were set manually. Linear superficial velocities $u_{S}$ were $0.5,1,2$, and $3 \mathrm{~m} / \mathrm{h}(0.5,1,1.5$, and $2 \mathrm{~m} / \mathrm{h}$ with dextran as viscosifier at $4.5 \mathrm{mPa} \cdot \mathrm{s}$ and $8 \mathrm{mPa} \cdot \mathrm{s})$. With each new mobile phase the column was equilibrated with a minimum of five column volumes. All measurements were corrected for extra-column contribution for each mobile phase viscosifier, viscosity, velocity, and target component, as described in [14].

\section{Measurement of partition coefficient $-K_{D}$}

The partition coefficient $K_{D}$ for sucrose and glycerol was calculated from the slopes of linear isotherms. Isotherms were measured via frontal analysis of breakthrough times with the staircase method via the refractive index (RI) as described by [18]. To the unviscosified phosphate buffer the viscous mobile phase was added in steps of $5 \%(\mathrm{v} / \mathrm{v})$, while the viscous mobile phase was at the same viscosifier concentration as the maximum used in the pulse injection measurements.

$K_{D}$ of all three mobile phase viscosifiers was additionally calculated with equation 4.4 from mean retention times measured from pulse injections of $80 \mu \mathrm{L}$ at $1 \mathrm{~m} / \mathrm{h}$ in unviscosified phosphate buffer as mobile phase. $V_{0}$ was measured with dextran $\left(2 \cdot 10^{6} \mathrm{Da}\right)$ and $V_{T}$ with $2 \%(\mathrm{v} / \mathrm{v})$ acetone. $K_{D}$ of dextran was measured from pulse injections and calculated with equation 4.4 . 


\section{Mobile phase viscosity}

Mobile phase viscosity was increased through addition of sucrose, glycerol, or dextran. Viscosity of different viscosifier concentrations in phosphate buffer were measured in a rheometer at $22^{\circ} \mathrm{C}$ and functions fitted to the data. Viscosity of sucrose solutions was fitted to equation 4.9, a modification of an empirical model [19]. Viscosity of glycerol solutions was estimated using equations 4.10 through 4.15 , as proposed by [20]. Viscosity of dextran solutions was fitted to equation 4.16, entirely based on measurements. Measurements, empirical models and fitted functions are shown in Fig. 4.11 which, along with equations 4.9 through 4.16, can be found in the supplementary material.

\section{Van Deemter curves and intraparticle diffusivity $D_{p}$}

Van Deemter curves, HETP as function of linear interstitial velocity $u_{L}$, were recorded for each target component and mobile phase. From the slope in the linear region of each van Deemter curve the intraparticle diffusivity $D_{p}$ was calculated as described in [7]. This method also takes the resistance to external mass transfer $k_{\text {film }}$ into account, which was calculated using the Wilson and Geankoplis relation. Non-interacting conditions were assumed. The confidence interval of $D_{p}$ was calculated from the propagated uncertainties of the slope of van Deemter curves and $k_{f i l m}$. The uncertainty of the slope was calculated from the standard error of the slope with a $95 \%$ confidence interval, the uncertainty of $k_{f i l m}$ was estimated assuming an uncertainty of $20 \%$ for $D_{m}$.

\section{Parameters for system size calculations}

The influence of the bulk viscosity on the system size is illustrated by separation of two minor components (target component and a component without affinity for the resin) in a viscous medium. For the calculation of column dimensions as function of viscosity, case parameters were set. All case parameters are given in Table 4.3. For the calculation of pressure drop a bed porosity of 0.34 was assumed, even though van Deemter curves at higher viscosities were recorded with lower bed porosities.

An initial stream volume of $10 \mathrm{~m}^{3} / \mathrm{h}$ and a viscosifier concentration of $1 \%(\mathrm{~m} / \mathrm{m})$ was assumed. Change in stream volume through water removal and subsequent increase in viscosity, was simulated by increasing concentration of viscosifying agent in the mobile phase while assuming a linear isotherm for all target components.

Table 4.3 - Case parameters for comparison of change in column dimensions with increased viscosity.

\begin{tabular}{r|rrr} 
Name & Symbol & Quantity & Unit \\
\hline Pressure drop & $\Delta \mathrm{p}$ & 5 & $\mathrm{bar}$ \\
Initial stream volume & $Q_{V}$ & 10 & $\mathrm{~m}^{3} / \mathrm{h}$ \\
Number of plates & $N$ & 100 & - \\
Particle size & $d_{p}$ & 800 & $\mu \mathrm{m}$ \\
Temperature & $T$ & 22 & ${ }^{\circ} \mathrm{C}$ \\
Bed porosity & $\varepsilon_{b}$ & 0.34 & -
\end{tabular}




\subsection{Results and discussion}

\subsubsection{Van Deemter curves at increased viscosities}

Chromatography in viscous solutions leads to a decrease in overall mass transfer, resulting in increased slopes of van Deemter curves, as shown for triglycine in phosphate buffer viscosified to 2.3 and $8.7 \mathrm{mPa} \cdot \mathrm{s}$ with sucrose in Fig. 4.2a and viscosified to 4.5 and $8.7 \mathrm{mPa} \cdot \mathrm{s}$ with glycerol in Fig. 4.2b. Recording elution peaks in viscous mobile phases, two main effects and several side effects were observed. The first main effect was the expected increase in band broadening due to increased mass transfer resistance with viscosity. The second main effect was the degree of band broadening increase was correlated to the viscosity inside the pores, rather than the viscosity of the bulk phase; a topic further discussed in section 4.4.2.

a)

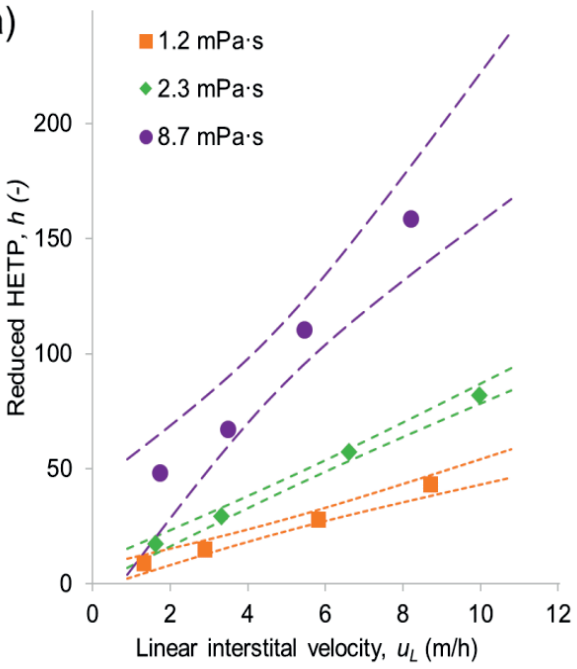

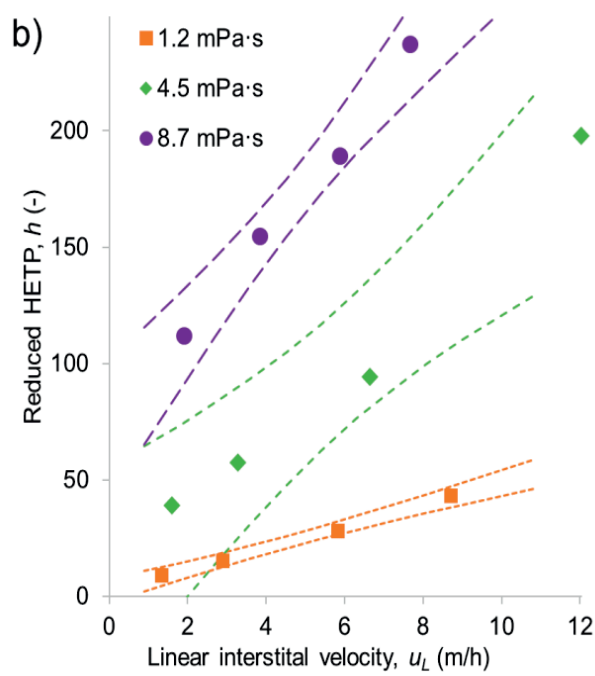

Fig. 4.2 - Van Deemter curves of reduced HETP $h$ over interstitial linear velocity $u_{L}$ for triglycine in phosphate buffer at $1.2 \mathrm{mPa} \cdot \mathrm{s}$ and (a) phosphate buffer containing sucrose at 2.3 and $8.7 \mathrm{mPa} \cdot \mathrm{s}$ and (b) phosphate buffer containing glycerol at 4.5 and $8.7 \mathrm{mPa} \cdot \mathrm{s}$. Lines indicate $95 \%$ confidence interval for linear regression of the slope.

The observed side effects challenged experimental HETP determination and ultimately decreased accuracy with increasing viscosity. At lower viscosities, measured data points of the van Deemter curve formed a straight line with little or no deviation, visible from the small $95 \%$ confidence interval displayed by dotted lines in Fig. 4.2. At higher viscosities of the mobile phase recorded van Deemter curves showed a larger spread of data points for expected linear behavior, leading to larger uncertainty in determined slopes, which could be improved through additional measurements. As pressure drop increased, the resin bed compressed slightly, resulting in different bed heights, bed porosities and retention volumes, which were corrected for in each case. Measured pressure drop was compared to the calculated pressure drop, which was calculated based on actual bed height and porosity with equation 4.7 (Fig. 4.3). In most cases measured pressure drop matched expected pressure drop fairly well. One measurement in glycerol, where measured pressure drop was far above expectation, led to a repacking of the column, as indicated in Fig. 4.3. Peak shapes changed from almost 
perfect symmetry to tailing and in some cases fronting peaks. Because of the focus of this work, the change in peak shape was not further analyzed, although it has an influence on HETP and an accurate description of the change in peak shape is important for application towards a practical separation. The elution peaks of acetone show an increase of retention volume with viscosity, with either sucrose or glycerol in the mobile phase (Fig. 4.13a), while all other retention volumes decrease (Fig. 4.13b, c, and d). We have found no plausible explanation for this observation. Last but not least baseline stability decreased, resulting in necessity for baseline correction in some cases, especially for $\gamma$-aminobutyric acid measured at a wavelength of $210 \mathrm{~nm}$ (Fig. 4.13b). Elution peaks of acetone, $\gamma$-aminobutyric acid, triglycine and glycyl-L-tyrosine at $u_{S}=1 \mathrm{~m} / \mathrm{h}$ in phosphate buffer, and mobile phase viscosified with sucrose at 2.3 and $8.7 \mathrm{mPa} \cdot \mathrm{s}$ and glycerol at 4.5 and $8.7 \mathrm{mPa} \cdot \mathrm{s}$ are added to the supplementary material (Fig. 4.13).

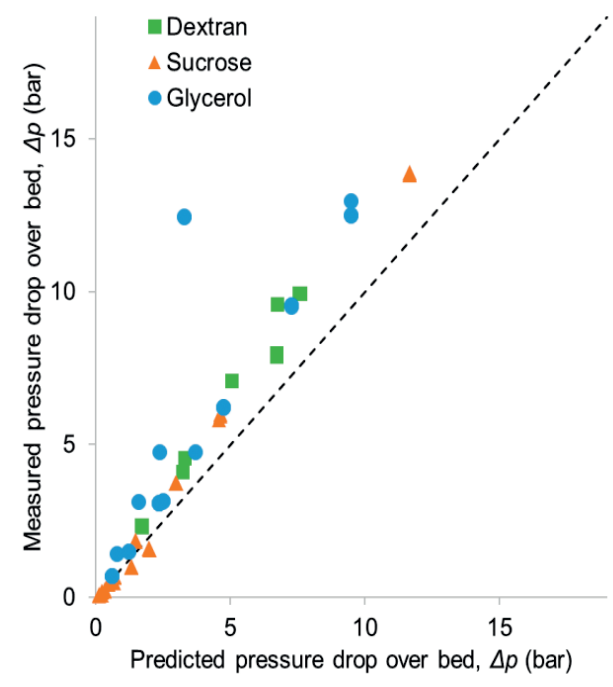

Fig. 4.3 - Measured pressure drop over the column bed over the calculated pressure drop from equation 4.7. Pressure drop of mobile phase viscosified with dextran was constantly larger than expected due to higher apparent viscosity.

For all investigated target components the following relationships were found: the larger the molecule, the steeper the slope of the van Deemter curve at low viscosity and greater the increase in slope with increased viscosity. Van Deemter curves of all target components showed similar behavior in terms of increasing confidence interval with viscosity (supplementary material, Fig. 4.14, Fig. 4.15, and Fig. 4.16).

Comparison of peak broadening for different target components at different viscosities is best demonstrated by plotting reduced HETP $h$ over reduced velocity $v$ which takes bulk diffusion coefficients into account (Fig. 4.4a for sucrose and Fig. 4.4b for glycerol in mobile phase). The slope of $h$ over $v$ increased with molecular size, but due to the correction for the bulk diffusion coefficient $D_{M, b u l k}$, displayed an almost linear behavior for each molecule with varying viscosities. But it is also evident from Fig. 4.4, that at high viscosity overall mass transfer resistance is not exactly proportional anymore to the diffusion coefficient in the bulk mobile phase, as slopes at $8.7 \mathrm{mPa} \cdot \mathrm{s}$ differ from slopes at lower viscosities. 

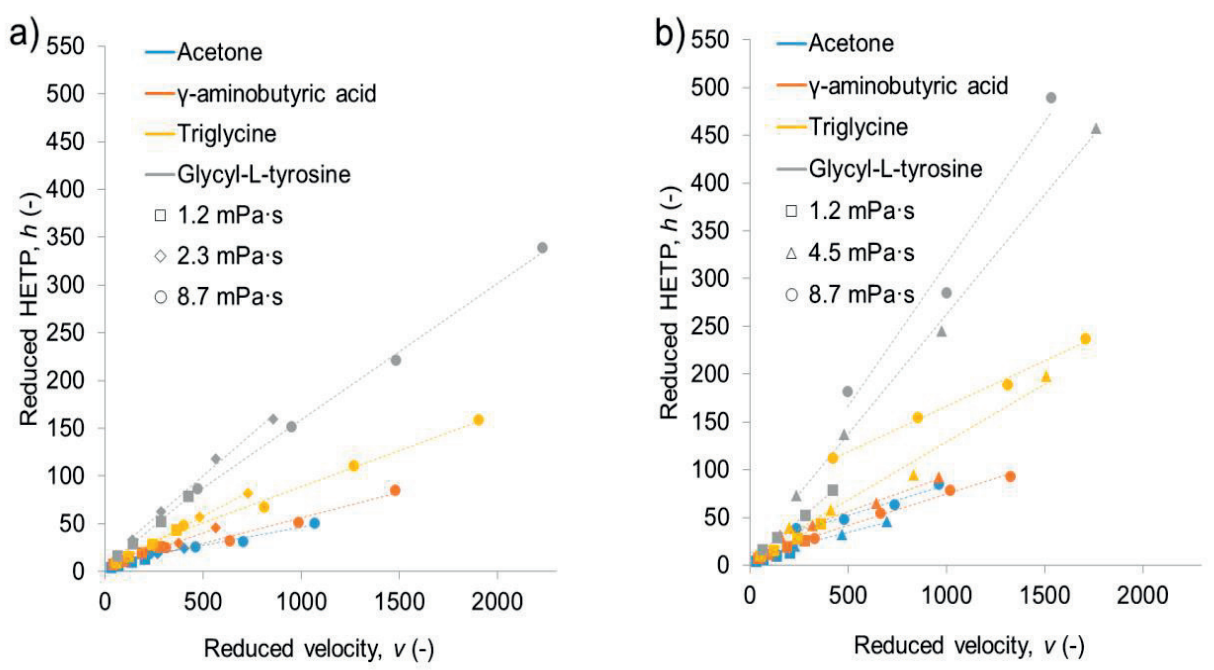

Fig. 4.4 - Reduced HETP $h$ over reduced velocity $v$ for pulse injections of acetone, $\gamma$-aminobutyric acid, triglycine, and glycyl-L-tyrosine in phosphate buffer at $1.2 \mathrm{mPa} \cdot \mathrm{s}$ and a) phosphate buffer containing sucrose at 2.3 and $8.7 \mathrm{mPa} \cdot \mathrm{s}$ and $\mathrm{b}$ ) phosphate buffer containing glycerol at 4.5 and $8.7 \mathrm{mPa} \cdot \mathrm{s}$.

\subsubsection{Influence of the partition coefficient of the viscosifying agent}

A direct comparison of van Deemter curves for mobile phases viscosified with sucrose and glycerol revealed a difference in slope at the same mobile phase bulk viscosity (round symbols for $8.7 \mathrm{mPa} \cdot \mathrm{s}$ in Fig. $4.4 \mathrm{a}$ and Fig. 4.4b). The same bulk viscosity resulted in a difference in slope and therefore a different resistance to overall mass transfer. From the slope of the van Deemter curve, the overall resistance to mass transfer was calculated, as described in [7], which takes into account both the mass transfer resistance inside the particle and outside the particle in the stationary film layer. The contribution of the film mass transfer resistance $k_{f i l m}$, as calculated by the Wilson and Geankoplis relation, to the overall mass transfer resistance was negligible. Since bulk diffusivities in sucrose and glycerol were almost identical and overall mass transfer was dominated by the contribution of transparticle mass transfer, it stands to reason that the major difference between mass transfer in mobile phases viscosified with sucrose or glycerol affected diffusion inside the particle pores. Inside the pore volume, steric restriction imposed by the pore structure lead to a different distribution of viscosifier molecules between external and internal particle space, resulting in different viscosities, and thus diffusivities, in the pore volume. The difference in viscosity inside the pore and dependence of overall mass transfer not only on bulk viscosity, but also the viscosifiers ability to penetrate pore volume was shown when dextran was introduced; a viscosifying agent too large to penetrate any pore volume.

The concentration of viscosifier molecules, and with that an averaged viscosity inside the pore volume, was calculated from the isotherms of sucrose and glycerol (Fig. 4.5). Since the isotherms are linear, the concentration can also be calculated via the partition coefficient $K_{D}$ (equation ). The measured $K_{D}$ values for sucrose and glycerol were 0.48 and 0.70 respectively. Measured $K_{D}$ from pulse injections match the slope of 
isotherms for sucrose and glycerol. The isotherm of dextran showed an increase in stationary phase concentration at higher mobile phase concentration (Fig. 4.5), which cannot be explained through pore penetration, as the dextran used is about an order of magnitude larger than the cut off of the resin. A pore concentration of zero, as measured via the partition coefficient was assumed for the remainder of the experiments with dextran in the mobile phase. Viscosity inside the pore volume, calculated from the partition coefficient, is plotted as a function of bulk viscosity in Fig. 4.6. In addition to glycerol and sucrose, Fig. 4.6 also shows the pore viscosity for dextran with a $K_{D}$ of 0 (no access to any pore volume) and the case for a $K_{D}$ of 1 (complete access to pore volume).

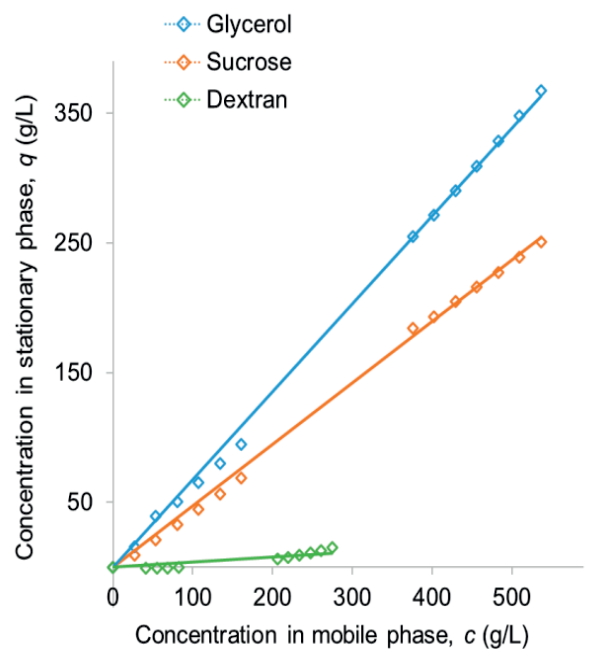

Fig. 4.5 - Isotherms of glycerol, sucrose and dextran.

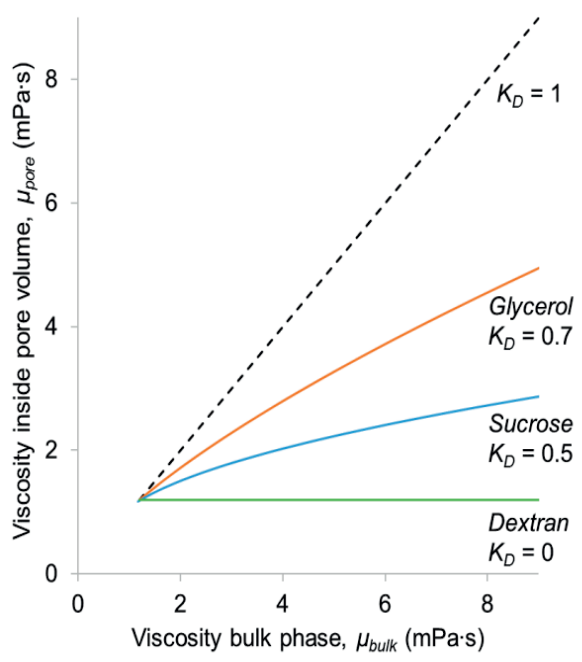

Fig. 4.6 - Change of viscosity in pore volume $\mu_{\text {pore }}$ as function of bulk phase viscosity $\mu_{\text {bulk }}$, based on measured partition coefficient $K_{D}$.

The dependence of mass transfer on the viscosity inside the pores is demonstrated by plotting the intraparticle diffusivity measured in mobile phase viscosified with either sucrose (closed symbols) or glycerol (open symbols) as a function of pore volume viscosity for four different target components (Fig. 4.7). The calculated intraparticle diffusivities are small compared to bulk diffusivities, however the range of $\Omega$ of 3 to $8 \%$ fits well with data measured for an upcoming publication and also with simulated data [21, 22].

By accounting for the partition coefficient of the mobile phase viscosifying agent the respective intraparticle diffusivities line up well for each molecule, independent of the mobile phase viscosifying agent used. In all measurements glycyl-L-tyrosine was retained by an unknown mechanism, therefore the calculated intraparticle diffusivity is larger than for triglycine at low intraparticle viscosity, based on their respective molecular size alone, the opposite relationship was expected. The dependence of mass transfer on the viscosity inside the pores is further demonstrated by viscosifying the mobile phase with dextran, a molecule so large it does not have any access to the pore volume $\left(K_{D}=0\right)$. The influence of the mobile phase viscosifying agents partition coefficient $K_{D}$ becomes visible when the relative intraparticle diffusivity of triglycine is plotted against $K_{D}$. Fig. 4.8 compares the intraparticle diffusivity measured at different viscosities due to glycerol, 
sucrose and dextran to intraparticle diffusivity measured in non-viscosified conditions. The reduction of $D_{p}$ is greatest for glycerol, the smallest viscosifier molecule with the largest $K_{D}$. At the same bulk mobile phase viscosity, $D_{p}$ is greater in mobile phase viscosified with sucrose because the viscosity in the pores is lower. For mobile phases viscosified with dextran to $4.5 \mathrm{mPa} \cdot \mathrm{s}$ and $8.0 \mathrm{mPa} \cdot \mathrm{s}$, no significant change in $D_{p}$ was measured because the viscosity in the pores stayed the same. Additionally to measured data, two calculated values for $D_{p}$ were added to Fig. 4.8 , based on the measured change of $D_{p}$ with pore viscosity (open symbols). The difference intraparticle diffusivity shows the dependence of mass transfer resistance on the accessible fraction of pore volume for the viscosifying agent. The error bars show the $95 \%$ confidence intervals based on the uncertainty of the van Deemter slopes. For acetone and $\gamma$-aminobutyric acid, measured intraparticle diffusivity in sucrose and glycerol behaved similar to triglycine. Intraparticle diffusivity is not the only mass transfer to be considered, but it is the main bottleneck to efficient column design in many chromatographic applications at scale. Accurate data of change in overall mass transfer with viscosity can be used to design chromatographic systems.

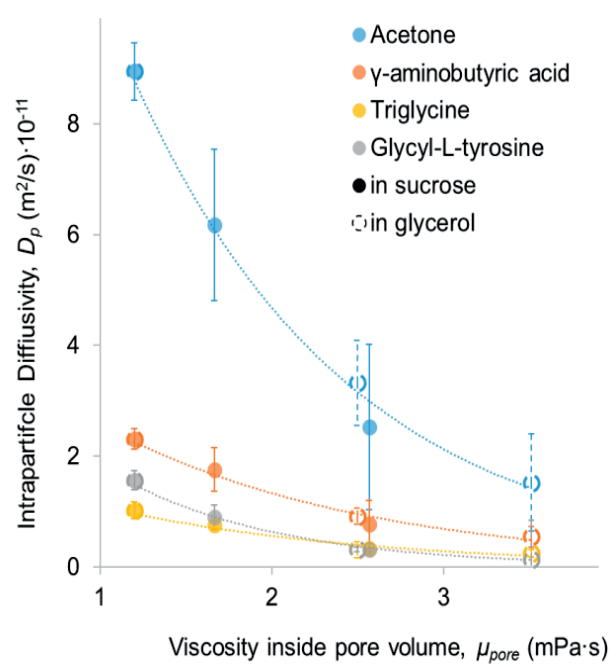

Fig. 4.7 - Intraparticle diffusivities of acetone, $\gamma$ aminobutyric acid, triglycine, and glycyl-L-tyrosine as function of viscosity inside pore volume due to sucrose (closed symbols) and glycerol (open symbols). Error bars show $95 \%$ confidence interval.

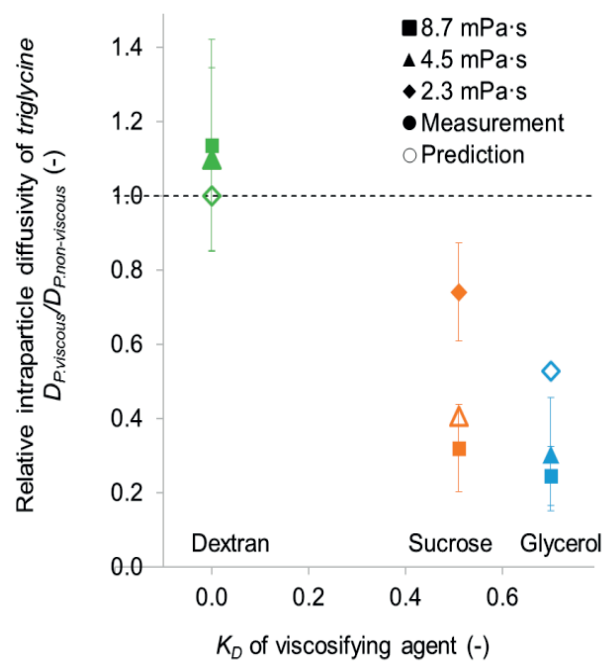

Fig. 4.8 - Intraparticle diffusivity $D_{p}$ of triglycine in mobile phases of $2.3 \mathrm{mPa} \cdot \mathrm{s}, 4.5 \mathrm{mPa} \cdot \mathrm{s}$, and $8.7 \mathrm{mPa} \cdot \mathrm{s}$ bulk viscosity with sucrose, glycerol, or dextran as viscosifying agent, relative to $D_{p}$ in phosphate buffer. Closed symbols represent measured $D_{p}$, open symbols show interpolated values, based on measured intraparticle diffusivities.

\subsubsection{System design based on bulk mobile phase viscosity}

System sizes were calculated for input streams with different viscosities caused by changes in viscosifier concentration. The change in concentration reflected on the stream volume, which was calculated for viscous streams of $8.7 \mathrm{mPa} \cdot \mathrm{s}$ diluted down to $1.2 \mathrm{mPa} \cdot \mathrm{s}$, on the basis of a throughput of $10 \mathrm{~m} / \mathrm{h}$ at $1.2 \mathrm{mPa} \cdot \mathrm{s}$. For the 
estimation of system size with changing viscosity due to changing concentration, three relationships needed to be known: viscosity of an input stream as a function of concentration, pressure drop over a column bed as function of viscosity and velocity, and HETP as function of viscosity and velocity. The first relationship can be found in literature for most mobile phases and is easily measured in other cases. The second relationship can be accurately calculated with equation 4.4 for non-compressible stationary phases, but the third relationship was not found in literature and was therefore measured in this work (section 4.4.1).

The influence of bulk viscosity on system size is illustrated in the following via the separation of two minor components (target component triglycine and a component which is not retained by the resin) in a viscous medium (sucrose solution). Three important assumptions were made for the design: first, the target component did not influence mobile phase viscosity. Second, the stationary-phase was non-compressible, leading to constant bed height and porosity, independent of column diameter and pressure drop. And third, the required number of plates stayed constant, even though differences in peak shape were observed. Analogue calculations for other target components and mobile phases viscosified through glycerol or dextran were made and their results will also be discussed. For a variety of bulk viscosities and a constant pressure drop $\Delta p$, the column length $L$ was calculated as a function of linear superficial velocity $u_{S}$ with equation 4.7 (solid lines in Fig. 4.9). Further, for the same bulk viscosities and a constant number of plates $N$, the required $L$ was calculated as a function of $u_{S}$ with equation 4.8 (striped lines in Fig. 4.9). Calculation of column length from $N$ required HETP as an input, which was measured for three different viscosities (as discussed in section 4.4.1). The slope of the van Deemter curve changed linearly with bulk viscosity, which allowed interpolation between measured data points to calculate the slope of van Deemter curves at any viscosity, within the measured range. Intersections in Fig. 4.9, marked with circles, show $L$ and $u_{S}$ for different viscosities at required operation parameters. With increasing viscosity, $L$ and $u_{S}$ were reduced, maintaining a constant pressure drop and number of plates. From the determined intersections in Fig. 4.9, column dimensions were calculated as described in section 4.2.2, taking into account an increase in stream volume, as concentration decreased through dilution.

Fig. 4.10a shows column volume as a function of bulk viscosity for mobile phases viscosified with sucrose, glycerol, and dextran and triglycine as target components, Fig. $4.10 \mathrm{~b}$ column area $A$ and column length $L$, for the same data. Change in column volume was dictated by the effect viscosity had on overall mass transfer. The change of pressure drop per column length was largely balanced through changes in the ratio of column area to column length.

With decreasing viscosity, from $8.7 \mathrm{mPa} \cdot \mathrm{s}$ until around $2.5 \mathrm{mPa} \cdot \mathrm{s}$, column volume stayed more or less constant. The minute changes in column volume at higher viscosities were well within the margin of error attributable to the uncertainty encountered when measuring van Deemter curves at higher viscosities. Dilution to viscosities lower than $2.5 \mathrm{mPa} \cdot \mathrm{s}$, led to drastic increase in column volume (Fig. 4.10a). In the region of low viscosities, relatively large changes in stream dilution and thus stream volume and viscosifier concentration, had little impact on feed viscosity and mass transfer resistance. In a scenario where streams are diluted before chromatographic separation, the results show dilution down to a viscosity of around $2.5 \mathrm{mPa} \cdot \mathrm{s}$ would have little to no effect on overall column volume. Dilution beyond $2.5 \mathrm{mPa} \cdot \mathrm{s}$ would result in drastically larger column volumes. 
For all target components, change in column volume with viscosity was similar, resulting in a more or less constant column volume at viscosities larger than approximately $2.5 \mathrm{mPa} \cdot \mathrm{s}$. Also for different viscosifiers, change in column volume followed the same overall trend, with differences in the range of 2 through $3 \mathrm{mPa} \cdot \mathrm{s}$. These differences are due to the difference in viscosity at the same viscosifier concentration and therefore stream volume reduction. With dextran as mobile phase viscosifier for example, measured van Deemter slopes are less sensitive to viscosity changes but viscosity is more sensitive to concentration changes. The sum of these two effects lead to a larger column volume with dextran as viscosifying agent in the range of 2 through $3 \mathrm{mPa} \cdot \mathrm{s}$, in comparison to sucrose or glycerol.

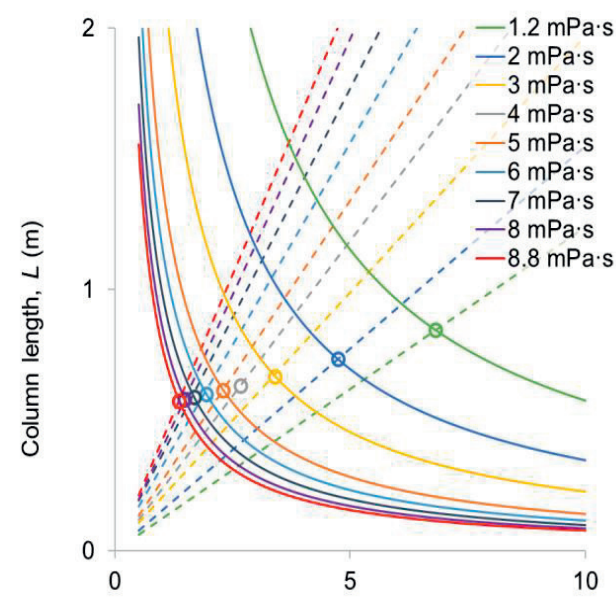

Superficial linear velocity, $u_{S}(\mathrm{~m} / \mathrm{h})$

Fig. 4.9 - Calculated column length $L$ for a range of bulk viscosities at different linear superficial velocities $u_{S}$. Solid lines present constant pressure drop $\Delta p$ (5 bar), dashed lines present a constant number of theoretical plates $N(N=100)$ for triglycine in sucrose. Intersections, marked with circles, show column length $L$ and linear velocity $u_{S}$ at which all operating conditions are met.

For viscosities larger than about $2.5 \mathrm{mPa} \cdot \mathrm{s}$, where overall column volume did not change with stream viscosity, changing viscosifier concentration still affected column length $L$ and column area $A$ (Fig. 4.10b).

With initial dilution of the stream, linear velocity increased such, that column area decreased in order to accommodate both increasing stream volume and mass transfer, while the length of the column increased. Diluting further than about $2.5 \mathrm{mPa} \cdot \mathrm{s}$, led to an increase in column area, required to accommodate the large stream volumes at low velocities. With larger viscosifying agents, columns became shorter and wider. 

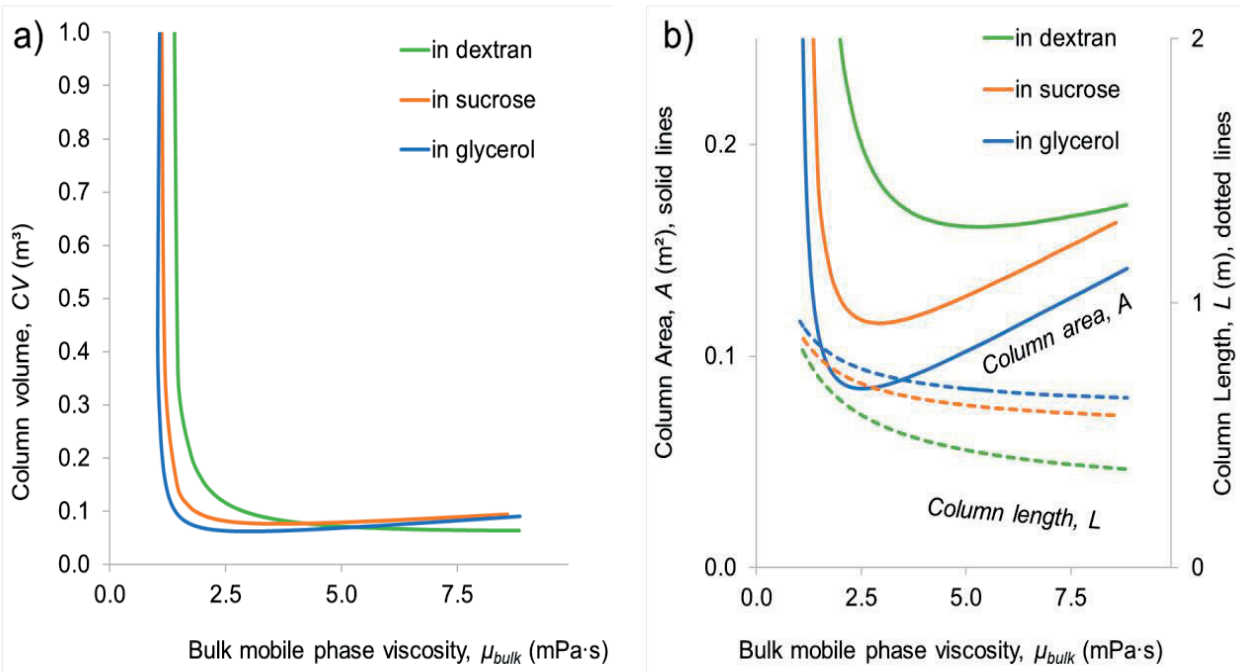

Fig. 4.10 - (a) Column volume $C V$ and (b) Column area $A$ (solid line) and column length $L$ (dotted line) as a function of bulk mobile phase viscosity $\mu_{\text {bulk }}$, for constant number of plates and pressure drop for triglycine viscosified with dextran, sucrose, glycerol.

\subsection{Conclusions}

This work shows HETP measurements in packed bed chromatography for a range mobile phase viscosities. The results were used to explain the dependence of HETP on the viscosity predominant in pore space, rather than in the bulk mobile phase and further to determine the change in column size and geometry for given process parameters when streams are concentrated. With increasing viscosity each van Deemter curve showed an increase in slope. Mass transfer in mobile phases with sucrose, glycerol or dextran differed, even with identical bulk viscosities, due to difference in viscosity in the pore volume. Larger molecules penetrate the pore volume to a smaller extend and have less influence on the diffusion inside the pores, which in preparative chromatography usually is the main resistance to mass transfer. Correction for viscosifier penetration into pore volume via the partition coefficient $K_{D}$, enabled the comparison and calculation of behavior for different viscosifying agents. The data showed that viscosity inside pore volume should be taken into account rather than only the viscosity of the bulk mobile phase. In case the target molecule is intrinsically viscous the difference between the viscosity inside and outside will be based on the same phenomena. The insights provided in this study can help taking viscosity differences into account when selecting a resin for a separation process. If the resin is selected such that it largely excludes the viscosity causing molecules from the pore space, mass transfer resistance inside the pores is reduced greatly and processes can be designed more efficiently.

Calculations for the influence of feed viscosity on system dimensions showed that column volume is more or less independent of stream viscosity in a viscosity range from $8.7 \mathrm{mPa} \cdot \mathrm{s}$ down to about $2.5 \mathrm{mPa} \cdot \mathrm{s}$. With dilution, columns get longer and thinner, but the overall volume stays near constant. Upon further dilution, 
column volume is increased drastically, as viscosity becomes less sensitive to concentration changes. The difference of column volume for different viscosifying agents is not pronounced as system size is mostly dependent on the slope of van Deemter curves as function of viscosity. Column dimensions however do differ between viscosifying agents, tending towards larger column areas and shorter columns with viscosifying molecule size. For the estimation of process economics, column geometry can be used as an indicator for unit costs, as the logarithm of unit costs generally behaves linearly to the logarithm of a unit operations required area [2].

\subsection{Acknowledgements}

The authors would like to thank Fleur Crielaard and Nina Kosten for their work on this project and Victor Aguirre Montesdeoca for his advice. This research took place within the framework of the Institute for Sustainable Process Technology ISPT. The authors would like to thank the ISPT for their support, together with Unilever (Vlaardingen, NL), FrieslandCampina Research (Amersfoort, NL), DSM (Delft, NL) and Cosun Food Technology (Roosendaal, NL) for their financial support and interest in this project.

\subsection{References}

1. H. Schmidt-Traub, M. Kaspereit, S. Engell, A. Susanto, A. Epping, A. Jupke, Model-Based Design, Optimization, and Control, Preparative Chromatography, Wiley-VCH Verlag GmbH \& Co. KGaA, 2012, pp. 425-518.

2. G. Guiochon, D.G. Shirazi, A. Felinger, A.M. Katti, Fundamentals of Preparative and Nonlinear Chromatography, Academic Press, 2006.

3. G. Guiochon, The limits of the separation power of unidimensional column liquid chromatography, Journal of Chromatography A, 1126 (2006) 6-49.

4. E.J. Fernandez, T.T. Norton, W.C. Jung, J.G. Tsavalas, A Column Design for Reducing Viscous Fingering in Size Exclusion Chromatography, Biotechnol.Prog., 12 (1996) 480-487.

5. A. Seidel-Morgenstern, M. Schulte, A. Epping, H. Schmidt-Traub, M. Schulte, A. SeidelMorgenstern, Fundamentals and General Terminology, Preparative Chromatography, Wiley-VCH Verlag GmbH \& Co. KGaA, 2012, pp. 7-46.

6. G. Carta, A. Jungbauer, Adsorption Kinetics, Protein Chromatography, Wiley-VCH Verlag GmbH \& Co. KGaA, 2010, pp. 161-199.

7. B. Coquebert de Neuville, A. Tarafder, M. Morbidelli, Distributed pore model for bio-molecule chromatography, Journal of Chromatography A, 1298 (2013) 26-34.

8. F. Gritti, G. Guiochon, Mass transfer kinetics, band broadening and column efficiency, Journal of Chromatography A, 1221 (2012) 2-40.

9. Y. Yao, A.M. Lenhoff, Determination of pore size distributions of porous chromatographic adsorbents by inverse size-exclusion chromatography, Estimation of Physicochemical Properties by Chromatographic and Electrophoretic Techniques, 1037 (2004) 273-282.

10. P. DePhillips, A.M. Lenhoff, Pore size distributions of cation-exchange adsorbents determined by inverse size-exclusion chromatography, Journal of Chromatography A, 883 (2000) 39-54.

11. R.C. Reid, J.M. Prausnitz, B.E. Poling, The properties of gases and liquids, (1987). 
12. S. Ergun, Fluid flow through packed columns, Chemical Engineering Progress, 48 (1952).

13. J.J. Stickel, A. Fotopoulos, Pressure-Flow Relationships for Packed Beds of Compressible Chromatography Media at Laboratory and Production Scale, Biotechnol.Prog., 17 (2001) 744-751.

14. A. Schultze-Jena, M.A. Boon, P.J.T. Bussmann, A.E.M. Janssen, A. van der Padt, The counterintuitive role of extra-column volume in the determination of column efficiency and scaling of chromatographic processes, Journal of Chromatography A, 1493 (2017) 49-56.

15. J.J. Baeza-Baeza, S. Pous-Torres, J.R. Torres-Lapasió, M.C. García-Álvarez-Coque, Approaches to characterise chromatographic column performance based on global parameters accounting for peak broadening and skewness, Journal of Chromatography A, 1217 (2010) 2147-2157.

16. A.J. Alexander, T.J. Waeghe, K.W. Himes, F.P. Tomasella, T.F. Hooker, Modifying conventional high-performance liquid chromatography systems to achieve fast separations with Fused-Core columns: A case study, Journal of Chromatography A, 1218 (2011) 5456-5469.

17. F. Gritti, A. Felinger, G. Guiochon, Influence of the errors made in the measurement of the extracolumn volume on the accuracies of estimates of the column efficiency and the mass transfer kinetics parameters, Journal of Chromatography A, 1136 (2006) 57-72.

18. J.A. Vente, H. Bosch, A.B. de Haan, P.J.T. Bussmann, Evaluation of sugar sorption isotherm measurement by frontal analysis under industrial processing conditions, Journal of Chromatography A, 1066 (2005) 71-79.

19. M.V. Galmarini, R. Baeza, V. Sanchez, M.C. Zamora, J. Chirife, Comparison of the viscosity of trehalose and sucrose solutions at various temperatures: Effect of guar gum addition, LWT - Food Science and Technology, 44 (2011) 186-190.

20. N.-S. Cheng, Formula for the Viscosity of a Glycerol-Water Mixture, Ind Eng Chem Res, 47 (2008) 3285-3288.

21. J.J. Meyers, O.K. Crosser, A.I. Liapis, Pore network modelling of affinity chromatography: determination of the dynamic profiles of the pore diffusivity of $\beta$-galactosidase and its effect on column performance as the loading of $\beta$-galactosidase onto anti- $\beta$-galactosidase varies with time, Journal of Biochemical and Biophysical Methods, 49 (2001) 123-139.

22. J.J. Meyers, A.I. Liapis, Network modeling of the intraparticle convection and diffusion of molecules in porous particles packed in a chromatographic column, Journal of Chromatography A, 827 (1998) 197-213. 


\subsection{Supplementary material}

\subsubsection{Viscosity estimation}

Bulk viscosity as function of concentration was measured in a rheometer for sucrose, glycerol and dextran solutions and basic functions fitted to the measured data (Fig. 4.11). Viscosity for sucrose solutions was calculated with equation 4.9 , based on [19].

$$
\mu_{\text {Sucrose }}=A_{0} \cdot e^{A_{1} \cdot c_{\text {Sucrose }}}+A_{2}
$$

With $c_{\text {Sucrose }}$ in $\%(\mathrm{~m} / \mathrm{m})$ and $A_{0}, A_{l}$, and $A_{2}$ are empirical parameters (with values of $0.48,0.064$, and 0.6 respectively) determined for best fit between measurement and calculated data.

For the calculation of viscosity in glycerol solutions, the empirical correlation detailed in equations 4.10 through 4.15 was used, as proposed by [20].

$$
\begin{aligned}
& \mu=\mu_{w}^{\alpha} \mu_{g}^{1-\alpha} \\
& \alpha=1-c_{\text {Glycerol }}+\frac{a b c_{\text {Glycerol }}\left(1-c_{\text {Glycerol }}\right)}{a c_{\text {Glycerol }}+b\left(1-c_{\text {Glycerol }}\right)} \\
& \mu_{w}=1.790 e^{\left(\frac{(-1230+T) T}{36100+360 T}\right)} \\
& \mu_{g}=12,100 e^{\left(\frac{(-1233+T) T}{9900+70 T}\right)} \\
& a=0.705-00017 T \\
& b=(4.9+0.036 T) a^{2.5}
\end{aligned}
$$

Viscosity estimation of dextran relied on empirical data measured in a rheometer. Equation 4.16 fit the measured data with $A_{0}=1.1693$ and $A_{l}=0.0725$ and $c_{\text {Dextran }}$ in $\%(\mathrm{~m} / \mathrm{m})$.

$$
\mu_{\text {Dextran }}=A_{0} \cdot e^{A_{1} \cdot c_{\text {Dextran }}}
$$




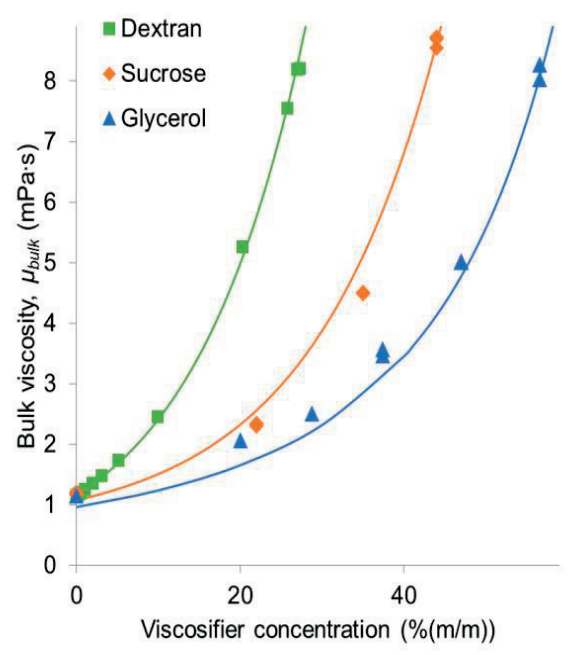

Fig. 4.11 - Measured viscosity as function of concentration for the viscosifying agents sucrose, glycerol and dextran as well as the fitted functions.

\subsubsection{Elution peaks}

\section{Baseline correction}

Fig. 4.12 shows recorded signals of $\gamma$-aminobutyric acid and their corresponding baseline corrected elution peaks, for a) phosphate buffer, b) $2.3 \mathrm{mPa} \cdot \mathrm{s}$ sucrose, c) $8.7 \mathrm{mPa} \cdot \mathrm{s}$ sucrose, d) $4.5 \mathrm{mPa} \cdot \mathrm{s}$ glycerol and e) $8.7 \mathrm{mPa} \cdot \mathrm{s}$ glycerol. For baseline correction, slope $m$ and intercept $b$ of the recorded signal between injection and shortly before peak start were analyzed. All recorded data points of the elution peak $y$ at time $t$ were corrected in the form $y_{\text {corrected }}(t)=y(t)-(m \cdot t+b)$. The start and endpoints of each elution peak were calculated automatically, where the signal reached $1 \%$ of the maximum signal intensity of the corrected peak. In cases were baseline inconsistency hindered this simple algorithm, the start and/or endpoints were set manually. 
a)

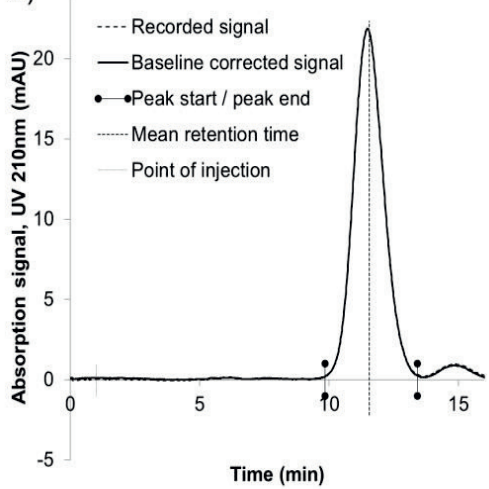

c)

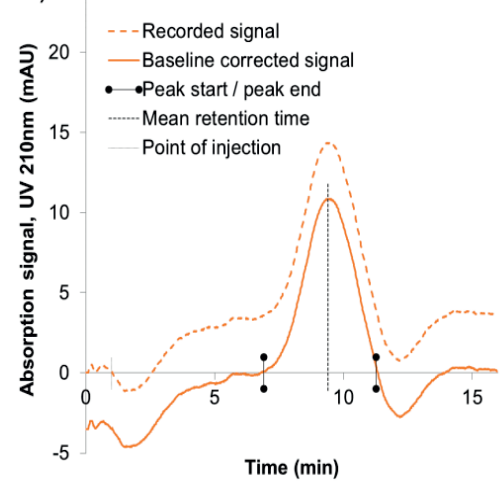

b)

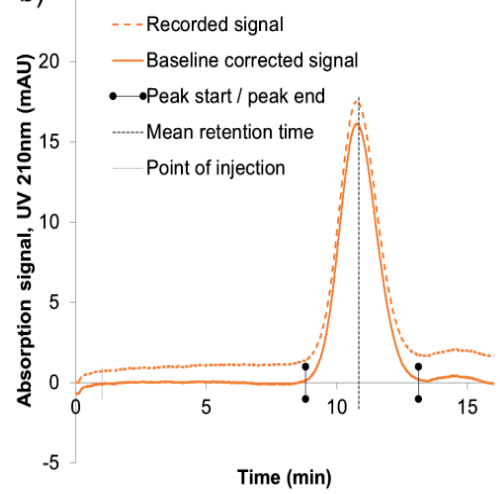

d)

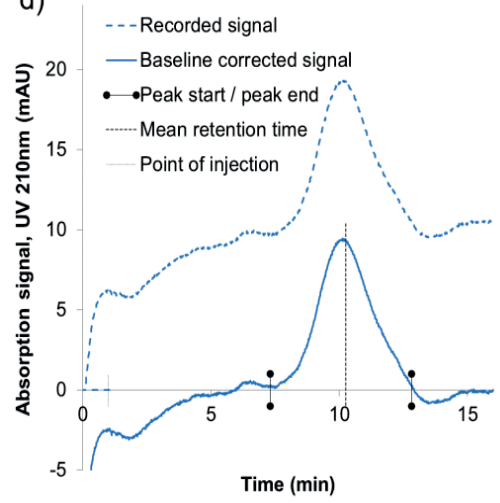

e)

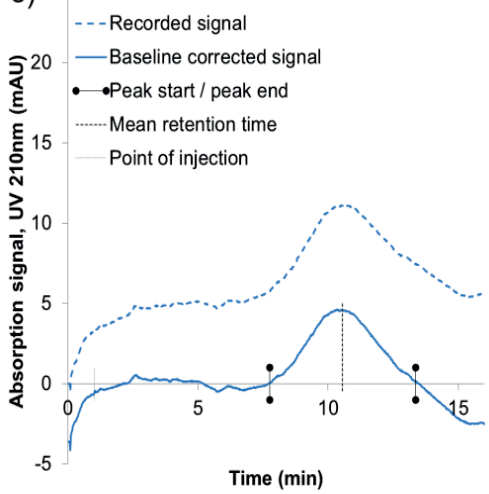

Fig. 4.12 - Recorded signals of $\gamma$-aminobutyric acid and their corresponding baseline corrected elution peaks, for a) phosphate buffer, b) $2.3 \mathrm{mPa} \cdot \mathrm{s}$ sucrose, c) $8.7 \mathrm{mPa} \cdot \mathrm{s}$ sucrose, d) $4.5 \mathrm{mPa} \cdot \mathrm{s}$ glycerol and e) $8.7 \mathrm{mPa} \cdot \mathrm{s}$ glycerol. 
Elution peak overlays
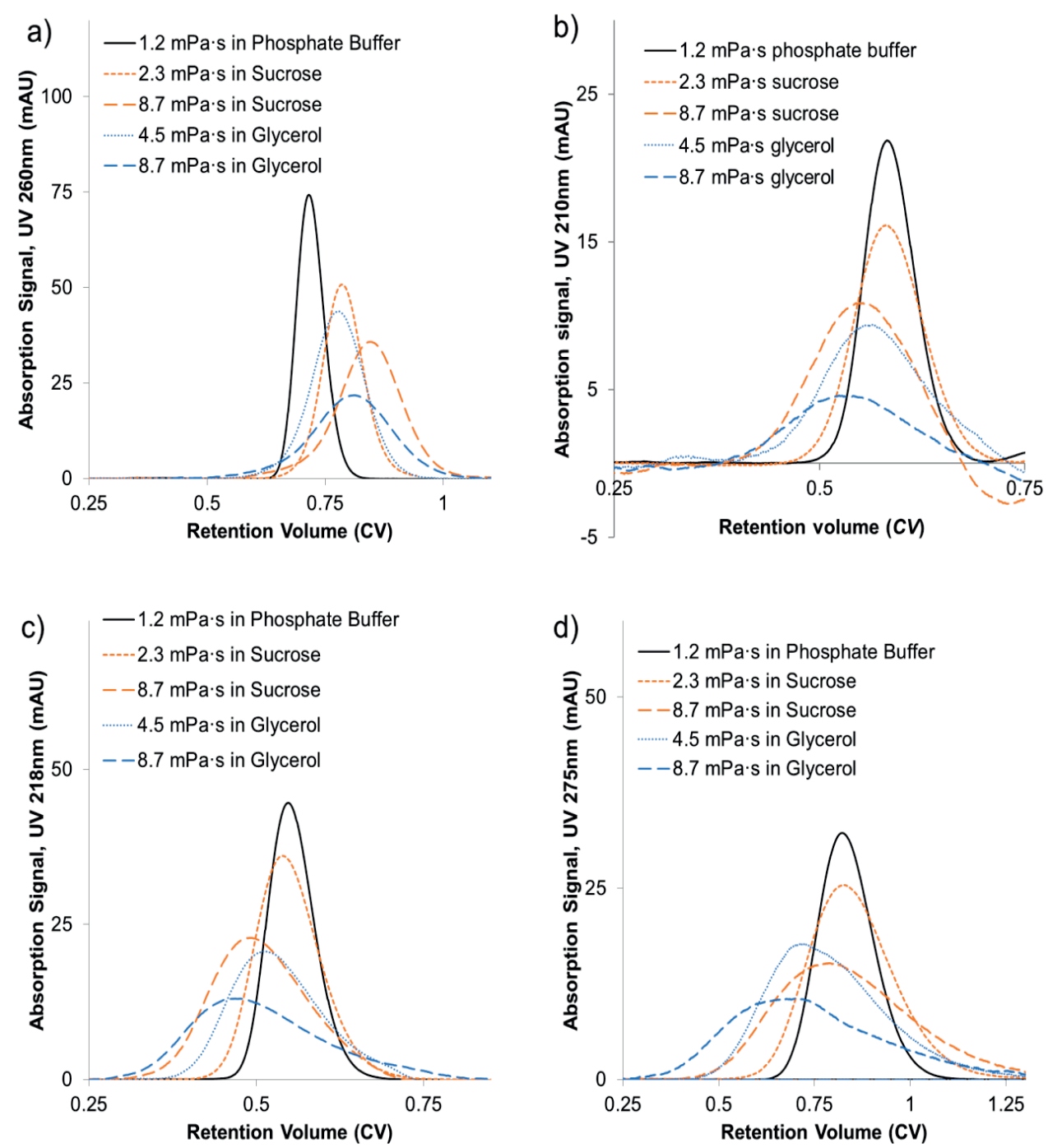

Fig. 4.13 - Overlaid chromatograms of ) acetone, b) $\gamma$-aminobutyric acid, c) triglycine, and d) glycyl-L-tyrosine in phosphate buffer, and mobile phase viscosified with sucrose at 2.3 and $8.7 \mathrm{mPa} \cdot \mathrm{s}$ and glycerol at 4.5 and $8.7 \mathrm{mPa} \cdot \mathrm{s}$ at $u_{S}=1 \mathrm{~m} / \mathrm{h}$. 
4.8.3 Van Deemter curves for sucrose in mobile phase
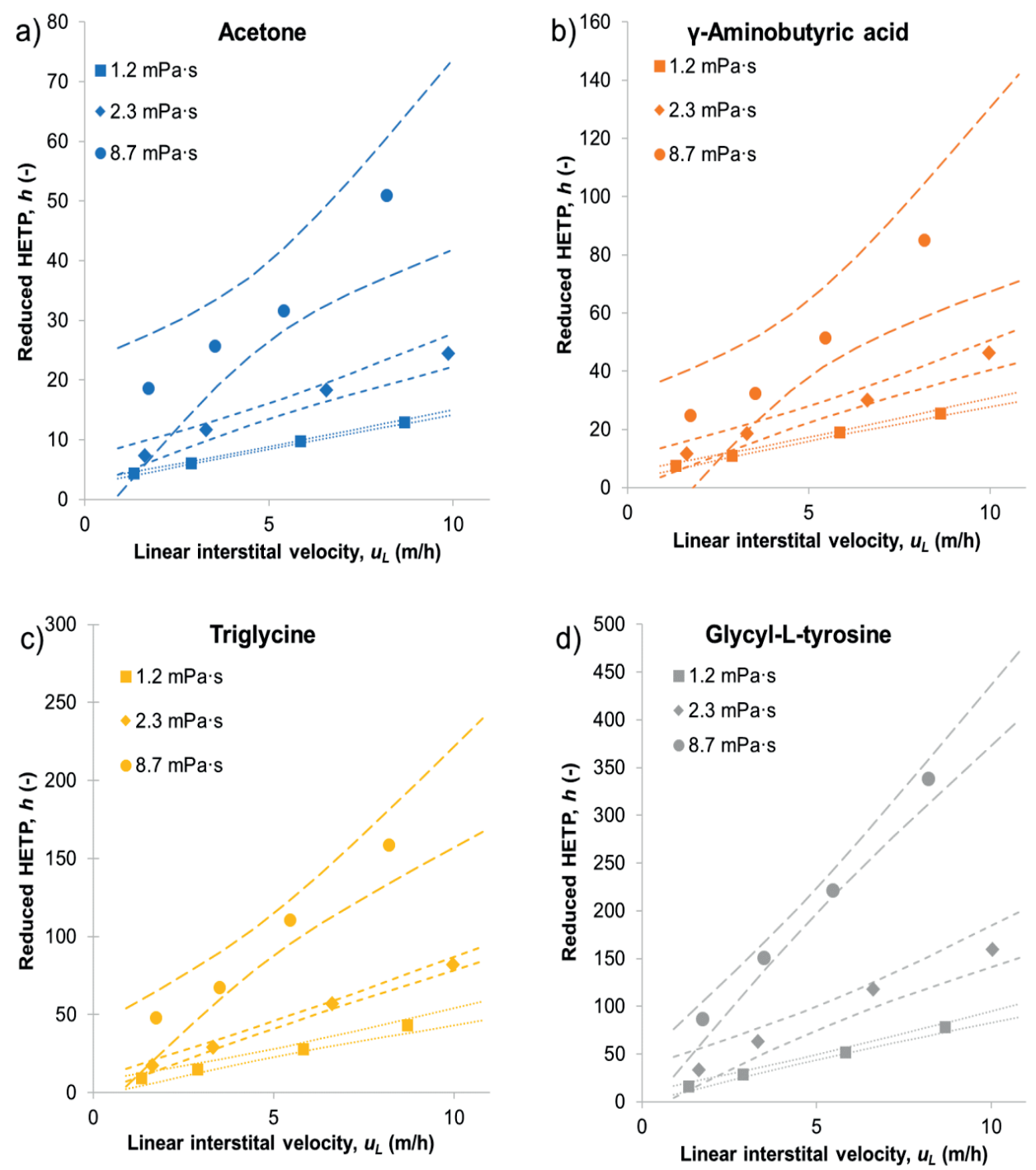

Fig. 4.14 - Van Deemter plots of reduced HETP $h$ over interstitial linear velocity $u_{L}$ for a) acetone, $b$ ) $\gamma$-aminobutyric acid, c) triglycine, and d) glycyl-L-tyrosine in phosphate buffer at $1.2 \mathrm{mPa} \cdot \mathrm{s}$ and phosphate buffer containing sucrose at 2.3 and $8.7 \mathrm{mPa} \cdot \mathrm{s}$. Lines indicate $95 \%$ confidence interval for linear regression of the slope. 
4.8.4 Van Deemter curves for glycerol in mobile phase
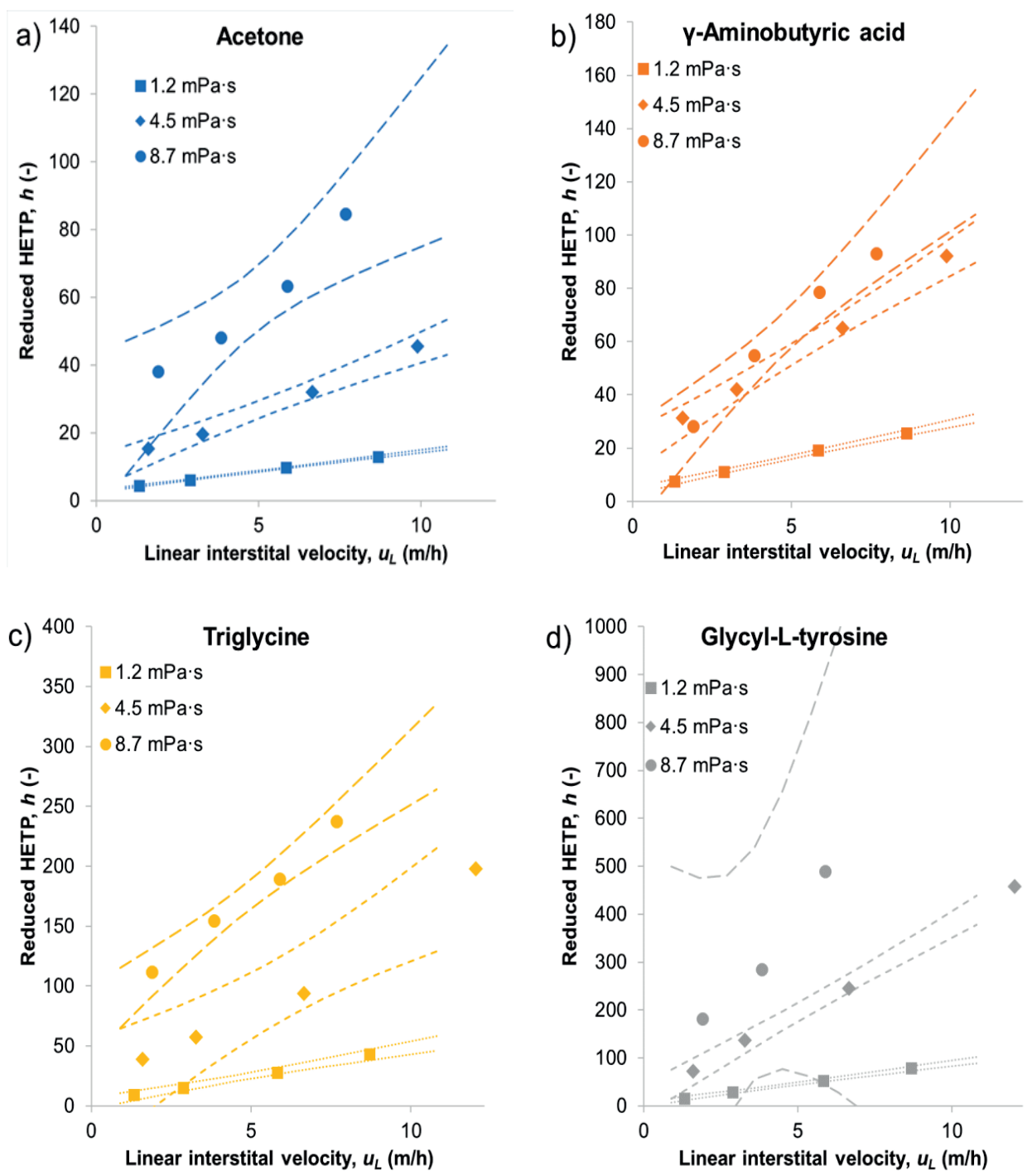

Fig. 4.15 - Van Deemter plots of reduced HETP $h$ over interstitial linear velocity $u_{L}$ for a) acetone, $b$ ) $\gamma$-aminobutyric acid, c) triglycine, and d) glycyl-L-tyrosine in phosphate buffer at $1.2 \mathrm{mPa} \cdot \mathrm{s}$ and phosphate buffer containing glycerol at 4.5 and $8.7 \mathrm{mPa} \cdot \mathrm{s}$. Lines indicate $95 \%$ confidence interval for linear regression of the slope. 
4.8.5 Van Deemter curves for dextran in mobile phase
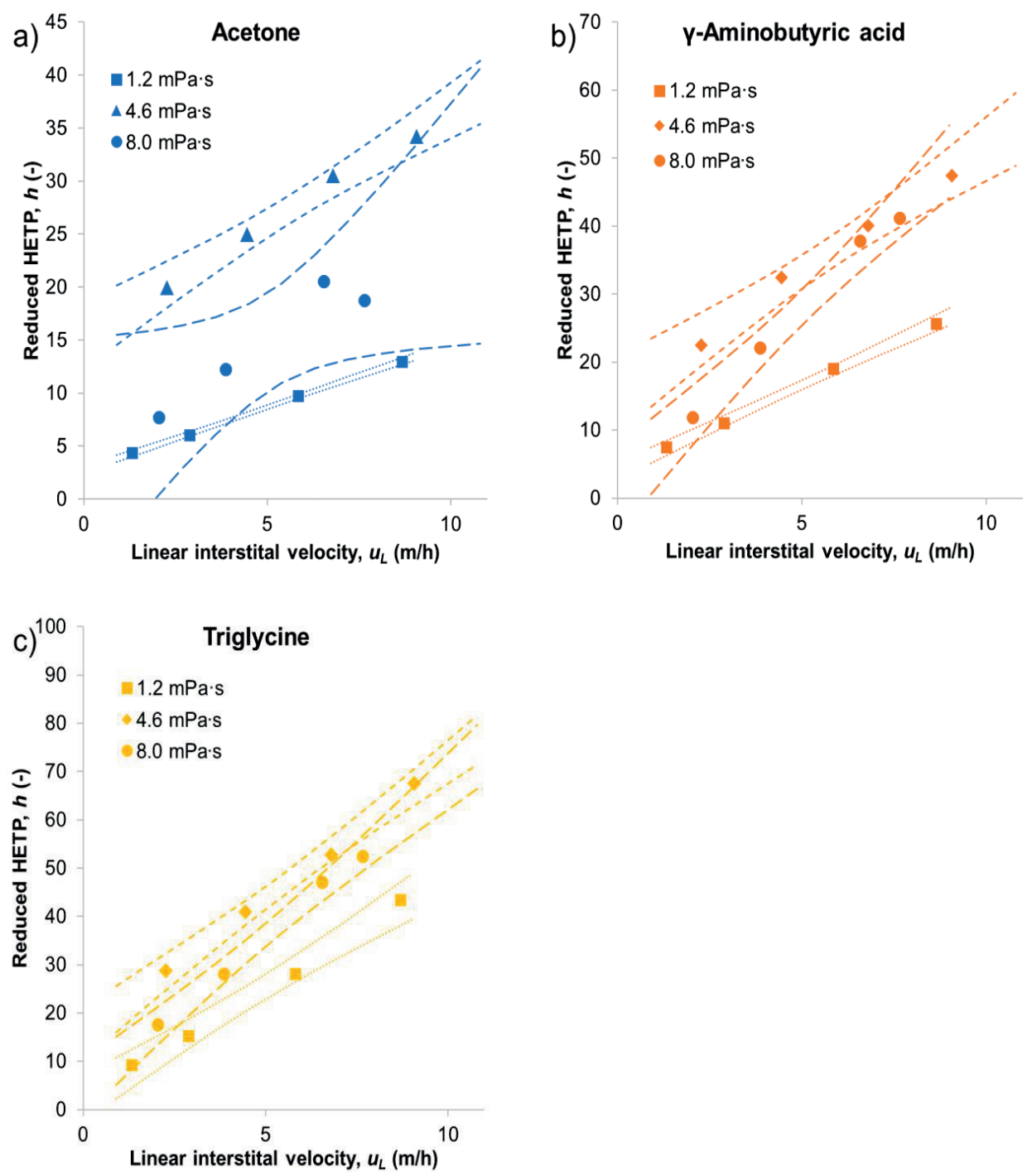

Fig. 4.16 - Van Deemter plots of reduced HETP $h$ over interstitial linear velocity $u_{L}$ for a) acetone, $b$ ) $\gamma$-aminobutyric acid, and c) triglycine, (no data for glycyl-L-tyrosine) in phosphate buffer at $1.2 \mathrm{mPa} \cdot \mathrm{s}$ and phosphate buffer containing dextran at $8.0 \mathrm{mPa} \cdot \mathrm{s}$. Lines indicate $95 \%$ confidence interval for linear regression of the slope. 



\section{Chapter 5}

\section{Elevated viscosities in a simulated moving bed using ion-exclusion chromatography: a case study on $\gamma$-aminobutyric acid recovery from \\ tomato serum}

This chapter has been submitted as:

A. Schultze-Jena, M.A. Boon, R.C. Vroon, P.J.Th. Bussmann, A.E.M. Janssen, A. van der Padt.

Elevated viscosities in a simulated moving bed using ion-exclusion chromatography: a case study on $\gamma$ aminobutyric acid recovery from tomato serum 


\begin{abstract}
Process streams of agro-food industries are often large and viscous. In order to fractionate such a stream the viscosity can be reduced by either a high temperature or dilution. In case of temperature sensitive components, temperature increase is not an option. Such streams are diluted prior to chromatographic fractionation, resulting in even larger volumes and high energy costs for sub-sequential water removal. The influence of feed viscosity on the performance of simulated moving bed SMB chromatography has been investigated. For this the recovery of a $\gamma$-aminobutyric acid rich fraction from tomato serum, with a restriction on the sugar content, is used as case study using ion-exclusion separation mechanism. This work addresses the SMB design, evaluates results from a pilot scale operation and uses these to calculate the productivity and water use at elevated feed concentration. The $S M B$ at the two higher feed viscosities (2.5 and $4 \mathrm{mPa} \cdot \mathrm{s}$ ) outperformed the $S M B$ at the lowest feed viscosity $(1 \mathrm{mPa} \cdot \mathrm{s})$ both in terms of water use and productivity. The behavior of the sugars for different feed viscosities can be described well by the model when the ratio of feed to eluent is used as dilution factor. The behavior of $\gamma$-aminobutyric acid in ion-exclusion chromatography is highly concentration dependent and the recovery could not be predicted with accuracy.
\end{abstract}




\subsection{Introduction}

Recovery of minor components from large agro-food streams offers a potential source for a variety of complex compounds. Due to the size of the available streams, these components are present in large quantities. Such components may be of value as functional ingredients in food products, however, they often are present in a mixture with less desired components (i.e. salts or mono- and disaccharides). For the food industry, such components are not only attractive with high purity, but also in the form of enriched fractions and clean-label components [1]. This offers a window of opportunity to design recovery processes, which are restricted by food industry specific requirements. Those requirements generally stem from a combination of process economics and functionality of target components.

Non-destructive technologies are required, which leave the processed stream unspoiled from the (mild) separation process so that all fractions may remain useable. Economic aspects mandate that the technologies are scalable and energy efficient. Component functionality requires mild treatment throughout the process, avoiding harsh chemicals or high temperatures, preferring the use of water as process aid.

Chromatographic processes are technically well suited for these type of separations, since they target specific interactions between required compounds and stationary phase and are scalable [2]. In the pharmaceutical industry chromatography is used to purify and fractionate complex molecules, such as proteins and peptides often on a relatively small scale. In the food industry the examples are limited to large scale applications such as high fructose corn syrup HFCS production and sucrose recovery from molasses [3]. Industrial-scaled preparative chromatography for food products could be done by using continuous multicolumn applications, like a simulated moving bed system with high productivities. Small and robust separation systems are required since they will use small amounts of rather expensive stationary phase, which is one of the main contributors to operation costs [4].

One way to reduce system size is the reduction of the feed volumetric flowrate. Feed streams can be reduced in volume by reduction of water content or by minimizing dilution of concentrated serums, syrups, extracts, etc. prior to the chromatographic separation. Generally, as the feed stream concentration decreases with dilution, resistance to mass transfer and pressure drop will decrease. With dilution feed stream volume increases. In this trade-off between feed stream size and mass transfer resistance, column volume increases at highly diluted feed streams in single column operations [5].

This research investigates the impact of feed stream concentration on the productivity and water use in a simulated moving bed $S M B$. The phenomena were studied using the case of obtaining an enriched $\gamma$-aminobutyric acid fraction from tomato serum using ion-exclusion chromatography. $\gamma$-Aminobutyric acid is a small amino acid and of interest as an additive in food products, it was separated from the saccharides present in the serum. 


\subsection{Materials and methods}

\subsubsection{Materials}

\section{Tomato serum}

Tomato serum was supplied by Unilever, NL. The serum was supplied in cooled containers at a concentration of $70^{\circ} \mathrm{Bx}$ and a viscosity of $271 \mathrm{mPa} \cdot \mathrm{s}$ (measured at $20^{\circ} \mathrm{C}$ ). The composition of tomato serum, as supplied by the manufacturer, is detailed in Table 5.1. The serum was diluted with Milli-Q water to reach concentrations of 7, 25, and $35^{\circ} \mathrm{Bx}$, which amounts to $1,2.5$ and $4 \mathrm{mPa} \cdot \mathrm{s}$ respectively. For isotherm measurements a model feed was used, as a defined and simplified version of the complex tomato serum.

Table 5.1 - Feed for the case study - tomato serum as supplied by Unilever, the model feed used for isotherm measurements, and the composition of the three diluted feed streams used for $S M B$ experiments.

\begin{tabular}{r|cc|cc|ccc|}
\multicolumn{2}{l|}{ Composition tomato serum $\left(\mathbf{7 0}^{\circ} \mathbf{B}\right)$} & \multicolumn{4}{|c|}{ Model feed $\left(\mathbf{7 0}^{\circ} \mathbf{B x}\right)$} & \multicolumn{3}{|c|}{ Composition diluted $[\mathbf{g} / \mathbf{k g} \mathbf{w w}]$} \\
\hline Component & {$[\mathrm{g} / \mathrm{kg} \mathrm{ww}]$} & {$[\% \mathrm{db}]$} & {$[\mathrm{g} / \mathrm{kg} \mathrm{ww}]$} & {$[\% \mathrm{db}]$} & $7^{\circ} \mathrm{Bx}$ & $25^{\circ} \mathrm{Bx}$ & $35^{\circ} \mathrm{Bx}$ \\
\hline Saccharides [g/kg ww] & & & & & & & \\
Fructose & 187.5 & 43.1 & 190.8 & 40.8 & 19.1 & 68.1 & 95.4 \\
Glucose & 166.5 & 38.2 & 169.8 & 36.3 & 17.0 & 60.6 & 84.9 \\
Sucrose & 6.5 & 1.5 & & & & & \\
\hline Minerals [g/kg ww] & & & & & & & \\
Monovalent $(\mathrm{K}, \mathrm{Na})$ & 28.4 & 6.5 & 31.0 & 6.6 & 3.1 & 11.1 & 15.5 \\
Divalent $(\mathrm{Ca}, \mathrm{Mg})$ & 2.6 & 0.6 & & & & & \\
\hline Organic acids [g/kg ww] & & & & & & & \\
Citrate & 35.1 & 8.1 & 35.1 & 7.5 & 3.5 & 12.5 & 17.6 \\
\hline Amino acids [g/kg ww] & & & & & & & \\
$\gamma$-Aminobutyric acid & 8.9 & 2.0 & 8.9 & 1.9 & 0.9 & 3.2 & 4.5 \\
Proteinaceous amino acids & 32.1 & 7.4 & 32.1 & 6.9 & 3.2 & 11.5 & 16.1
\end{tabular}

Stationary phase

Dowex 50WX4, a cation exchange resin, was used as stationary phase. The mean particle diameter (106 $\mu \mathrm{m})$ was measured with a Mastersizer 2000 (Malvern, UK). For the isotherm measurements and SMB pilot experiments, the stationary phase was equilibrated with an ion solution resembling the cationic composition of tomato serum: $38.35 \mathrm{~g} / \mathrm{L} \mathrm{KCl}, 3.28 \mathrm{~g} / \mathrm{L} \mathrm{CaCl} 2 \cdot 2 \mathrm{H}_{2} \mathrm{O}$, and $8.11 \mathrm{~g} / \mathrm{L} \mathrm{MgCl}_{2} \cdot 6 \mathrm{H}_{2} \mathrm{O}$.

\section{Chromatographic equipment}

For single column measurements a Wellchrom set-up with a K-1001 gradient HPLC pump, combined with a dynamic mixer and injection valve, was used. Detection via a K-2600 UV detector and a CM 2.1S conductivity detector; all from Knauer, Germany. Additional detection through a RI-502 RI detector from Shodex, Japan. Furthermore, a F25 MP water-bath (Julabo, Germany) controlled the temperature in the column jacket and a mini Cori-Flow flowmeter (Bronkhorst, The Netherlands) measured the flow rate after the detector. Elution profiles were measured in a Götec Superformance 300-10 column (300 x $10 \mathrm{~mm})$, packed to a bed height of $26.8 \mathrm{~cm}$ at a superficial velocity of $1.11 \mathrm{~m} / \mathrm{h}$. 
The $S M B$ pilot setup was built from six slurry packed Götec Superformance 300-16 columns (300 x $16 \mathrm{~mm}$ ) with tefzel capillaries of $35 \mathrm{~cm}$ lengths and ID $0.5 \mathrm{~mm}$, including flow adapter with frits and filter (all Götec, Germany). The columns were connected in series in a 2/1/2/1 configuration as shown in Fig. 5.1, with $26.6 \mathrm{~cm}$ average bed height, and $53.4 \mathrm{~mL}$ average bed volume. Water jackets of all columns were connected in line with a F25 MP water bath (Julabo, Germany) to control the column temperature at $20^{\circ} \mathrm{C}$. Four pumps, two for delivery of eluent (Milli-Q water) and feed (tomato serum) and two for extraction of raffinate and extract flows, all up to $50 \mathrm{~mL} / \mathrm{min}$ (Knauer, Germany). One pump $(250 \mathrm{~mL} / \mathrm{min})$ for recycling eluent from $S M B$ section 4 to section 1 (Knauer, Germany). Five multi-position valves (Knauer, Germany) for distribution of inlets (eluent and feed) and outlets (raffinate, extract and recycle) over the $S M B$ columns.

The $S M B$ was inline monitored at the extract and raffinate outlets using refractive index K-2401 $R I$ detectors (Knauer, Germany) and conductivity (GE Healthcare, USA) detectors respectively. Pump flows were monitored with inline mini CORI-FLOW flow meters (Bronkhorst, The Netherlands) at the pump outlets. Temperature of two column outlets were measured with inline thermocouples.

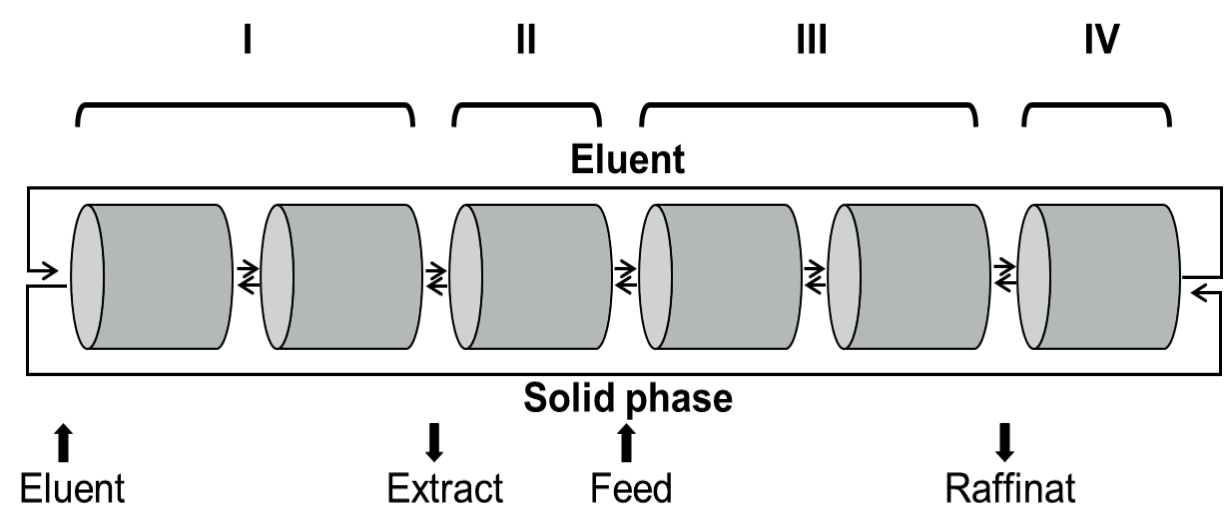

Fig. 5.1 - Schematic drawing of six column SMB set up. Sections marked with Roman numerals, schematic entry and exit ports at the bottom.

\subsubsection{Methods}

\section{Column characterization}

In preparation of the $S M B$ pilot experiments six columns were packed and characterized by pulse experiments. Prior to column packing the resin was conditioned with a $\mathrm{K} / \mathrm{Ca} / \mathrm{Mg}$ solution, to avoid swelling or shrinking of the stationary phase during operation. Columns were packed in Milli-Q, followed by bed compression with a $4 \mathrm{mPa} \cdot \mathrm{s}$ sugar solution at $15 \mathrm{~mL} / \mathrm{min}(450 \mathrm{~cm} / \mathrm{h})$. Next the columns were characterized by measuring bed height, porosities (bed and total porosity), and distribution coefficients of target components (glucose and minerals). It was shown that all columns were uniformly packed, by comparing pulse elution profiles, bed porosities, and distribution coefficients. 


\section{Isotherm measurement}

The linear isotherms of fructose and glucose were determined from pulse injections. The non-linear isotherm of $\gamma$-aminobutyric acid had to be measured via a different method and was determined from frontal analysis of breakthrough times as described in [6]. All isotherm measurements were carried out in mobile phase of three viscosities: 1,4 and $12.5 \mathrm{mPa} \cdot \mathrm{s}$, corresponding to 7,35 and $50^{\circ} \mathrm{Bx}$ tomato serum respectively. To increase viscosity fructose, glucose and sucrose were used in the same ratio as in tomato serum (35\% total sugars for $4 \mathrm{mPa} \cdot \mathrm{s}$ and $51 \%$ total sugars for $12.5 \mathrm{mPa} \cdot \mathrm{s}$ ).

\section{Chromatographic analysis}

The lumped kinetic model was used to describe mass transfer (equation 5.1), based on [7].

$$
\text { HETP }=\frac{2 D_{L}}{u_{L}}+\frac{2 u_{L}}{k_{\text {overall }} \cdot \frac{1-\varepsilon_{b}}{\varepsilon_{b}}} \cdot\left(\frac{k_{1}}{1+k_{1}}\right)^{2}
$$

$\operatorname{HETP}(\mathrm{m})$ is the height equivalent to a theoretical plate, measured from pulse experiments as described in [8]. $D_{L}$ is the axial diffusion coefficient $\left(\mathrm{m}^{2} / \mathrm{s}\right)$ in the mobile phase, which combines longitudinal diffusion and eddy dispersion in the moving eluent [9], $u_{L}$ is the interstitial linear velocity $(\mathrm{m} / \mathrm{s}), k_{\text {overall }}$ the lumped kinetic factor (1/s), $\varepsilon_{b}$ bed porosity (-), and $k_{l}$ the zone retention factor (-).

From the slopes of the linear part of van Deemter curves, in HETP $(\mathrm{m})$ over interstitial linear velocity $u_{L}(\mathrm{~m} / \mathrm{s})$, the lumped kinetic factor $k_{\text {overall }}(1 / \mathrm{s})$ was calculated with equation 5.2 [10].

$$
k_{\text {overall }}=\frac{\frac{2}{\frac{1-\varepsilon_{b}}{\varepsilon_{b}}} \cdot\left(\frac{k_{1}}{1+k_{1}}\right)^{2}}{\left(\frac{H E T P}{u_{L}}\right)}
$$

The zone retention factor $k_{l}$ calculated from equation 5.3, with the particle porosity $\varepsilon_{p}$ and the slope of the isotherm $\frac{\partial q}{\partial c}$, based on [7].

$$
k_{1}=\frac{1-\varepsilon_{b}}{\varepsilon_{b}}\left(\varepsilon_{p}+\left(1-\varepsilon_{p}\right) \frac{\partial q}{\partial c}\right)
$$

Intraparticle diffusivity $D_{p}\left(\mathrm{~m}^{2} / \mathrm{s}\right)$ was then calculated from equation 5.4 [10].

$$
D_{p}=\frac{r_{p}{ }^{2}}{15\left(\frac{1}{k_{\text {overall }}}-\frac{r_{p}}{3 \cdot k_{\text {film }}}\right)}
$$

With $r_{p}$ particle radius (m) and the resistance to mass transfer through the stagnant film layer $k_{f i l m}(\mathrm{~m} / \mathrm{s})$, calculated as a function of reduced velocity $v=\left(2 \cdot r_{p} \cdot u_{L}\right) / D_{m}(-)$ from the correlation of Wilson and Geankoplis [11] as shown in equation 5.5. 


$$
k_{f i l m}=\frac{1.09}{\varepsilon_{b}} \frac{D_{m}}{2 \cdot r_{p}} v^{1 / 3}
$$

\section{Viscosity measurement}

Mobile phase viscosity was measured with a Physica MCR 301 rheometer (Anton Paar, Austria) at $20^{\circ} \mathrm{C}$.

\section{SMB design}

A $S M B$ design model was used based on the lumped kinetic model. Six columns were distributed over the four sections of the $S M B$ in a 2/1/2/1 configuration. First, an initial estimate of flows, size of the columns, and switch time, was obtained from the triangle theory [12] was used. In a second step the design was optimized in gProms Modelbuilder 5.1.1. dynamic optimization routine, by finding ideal velocities for each section. The optimization objective was the maximization of feed flow over water input and the constrains were a 95\% removal of sugar and $\gamma$-aminobutyric acid recovery of $99 \%$. In these design calculations and to facilitate evaluation of practical results, the same pressured drop in the system for all viscosities was maintained (4 bar per column). All concentrations were calculated after 15 cycles of the entire system, well into steady state operation. $\gamma$-Aminobutyric acid recovery was calculated with equation 5.6, from mineral concentration in the raffinate $c_{\text {mineral.raff }}\left(\mathrm{kg} / \mathrm{m}^{3}\right)$, raffinate flow rate $q_{\text {raff }}\left(\mathrm{m}^{3} / \mathrm{h}\right)$, feed concentration $c_{\text {mineral.feed }}\left(\mathrm{kg} / \mathrm{m}^{3}\right)$, and feed flow rate $q_{\text {feed }}\left(\mathrm{m}^{3} / \mathrm{h}\right)$.

$$
\text { Recovery } y_{\gamma \text {-aminobutyric acid }}=\frac{c_{\text {mineral.raff }} \cdot q_{\text {raff }}}{c_{\text {mineral.feed }} \cdot q_{\text {feed }}} \cdot 100 \%
$$

Sugar removal was calculated with equation 5.7, from summed glucose and fructose concentration in the extract $c_{\text {sugar.extr }}\left(\mathrm{kg} / \mathrm{m}^{3}\right)$, extract flow rate $q_{\text {extr }}\left(\mathrm{m}^{3} / \mathrm{h}\right)$, summed glucose and fructose concentration in the raffinate $c_{\text {sugar.raff }}\left(\mathrm{kg} / \mathrm{m}^{3}\right)$, and raffinate flow rate $q_{\text {raff }}\left(\mathrm{m}^{3} / \mathrm{h}\right)$.

$$
\text { Removal }_{\text {sugars }}=\frac{c_{\text {sugar.extr }} \cdot q_{\text {extr }}}{c_{\text {sugar.extr }} \cdot q_{\text {extr }}+c_{\text {sugar.raff }} \cdot q_{\text {raff }}} \cdot 100 \%
$$

Productivity was calculated from equation 5.8 with feed concentration of $\gamma$-aminobutyric acid $c_{\gamma \text {-aminobutyric acidfeed }}\left(\mathrm{kg} / \mathrm{m}^{3}\right)$, the recovery of $\gamma$-aminobutyric acid (-), the feed flow rate $q_{\text {feed }}\left(\mathrm{m}^{3} / \mathrm{h}\right)$ and the system volume $V_{S M B}$ (volume of six columns, $\mathrm{m}^{3}$ ).

$$
\text { Productivity }=\frac{c_{\gamma \text {-aminobutyric acid.feed }} \cdot \text { Recovery }_{\gamma \text {-aminobutyric acid }} \cdot q_{\text {feed }}}{V_{S M B}}
$$

Water use was calculated as the sum of eluent (water input) and water required for feed dilution. Water required for feed dilution was calculated with equation 5.9 from the feed stream $q_{\text {feed }}\left(\mathrm{m}^{3} / \mathrm{h}\right)$ and dilution factor $D F(-)$.

$$
\text { Water for dilution }=q_{\text {feed }}-\frac{q_{\text {feed }}}{D F}
$$

With dilution factors of $10,2.2$, and 2 , concentrated tomato serum $\left(70^{\circ} \mathrm{Bx}\right)$ was diluted to viscosities of $1,2.5$, and $4 \mathrm{mPa} \cdot \mathrm{s}$ respectively. 


\section{SMB operation}

For each feed viscosity three experiments were performed in which the switch time was adjusted (Table 5.2) and the flows were kept constant. For each feed viscosity the optimal switch time calculated was the lowest switch time. During the start-up of each experimental series the flows were adapted in such a way that the pressure drop in the first section was approximately 4 bar.

Table 5.2 - Switch time ( $\mathrm{min})$ in the nine experiments.

\begin{tabular}{r|c|c} 
Feed viscosity & Experiment & Switch time [min] \\
\hline $1 \mathrm{mPa} \cdot \mathrm{s}\left(7^{\circ} \mathrm{Bx}\right)$ & $1-3$ & $1.25-1.38-1.44$ \\
$2.5 \mathrm{mPa} \cdot \mathrm{s}\left(25^{\circ} \mathrm{Bx}\right)$ & $4-6$ & $1.57-1.72-1.80$ \\
$4 \mathrm{mPa} \cdot \mathrm{s}\left(35^{\circ} \mathrm{Bx}\right)$ & $7-9$ & $2.00-2.20-2.30$
\end{tabular}

\section{Viscosity estimation}

The viscosity was estimated as an average over the system by using the ratio of feed and eluent (water) as dilution factor. The so estimated viscosity was compared to the viscosity calculated from the measured pressure drop. The pressure drop was measured with two EZG10 pressure sensors (Knauer, Germany) over one column which changed position with each column switch. During steady state operation, the viscosity in the system was estimated via the average pressure drop, using the Ergun equation (equation 5.10), with pressure drop $\Delta p(\mathrm{~Pa})$, column length $L(\mathrm{~m})$, mobile phase viscosity $\mu_{\text {bulk }}(\mathrm{Pa} \cdot \mathrm{s})$, particle diameter $d_{p}(\mathrm{~m})$, bed porosity $\varepsilon_{b}(-)$, mobile phase density $\rho\left(\mathrm{kg} / \mathrm{m}^{3}\right)$, and superficial linear velocity $u_{S}(\mathrm{~m} / \mathrm{s})[13]$.

$$
\Delta p=\frac{150 \mu_{b u l k}}{d_{p}^{2} L} \frac{\left(1-\varepsilon_{b}\right)^{2}}{\varepsilon_{b}{ }^{3}} u_{S}+\frac{1.75 \rho}{d_{p} L} \frac{\left(1-\varepsilon_{b}\right)}{\varepsilon_{b}{ }^{3}} u_{S}^{2}
$$

The total sugar concentration within each column was also analyzed at the end of experiments for each feed viscosity.

\subsection{Results and discussion}

\subsubsection{Simulated moving bed - design}

Tomato serum was analyzed for its key components (Table 5.1). The elution profile of each key component was recorded from pulse injections, an overlay of all elution profiles, recorded via the refractive index $R I$, is given in Fig. 5.2a. The profiles of the saccharides and $\gamma$-aminobutyric acid were well separated, the later showed little to no retention, eluting together with the minerals. Fig. 5.2b shows the same pulse injections as Fig. 5.2a, with an overlay of diluted (1:10) tomato serum. The profile of tomato serum was recorded via $R I$ and electric conductivity $E C$. The $R I$ overlay showed that the key components of the complex tomato serum are well represented by the individually injected components. The $E C$ overlay showed that almost all charged components are in the first peak. These charged components had almost no retention, and were thus excluded from the intraparticle pore volume. Based on these results it was decided, for practical reasons, to use minerals as an indicator for $\gamma$-aminobutyric acid during the design. 

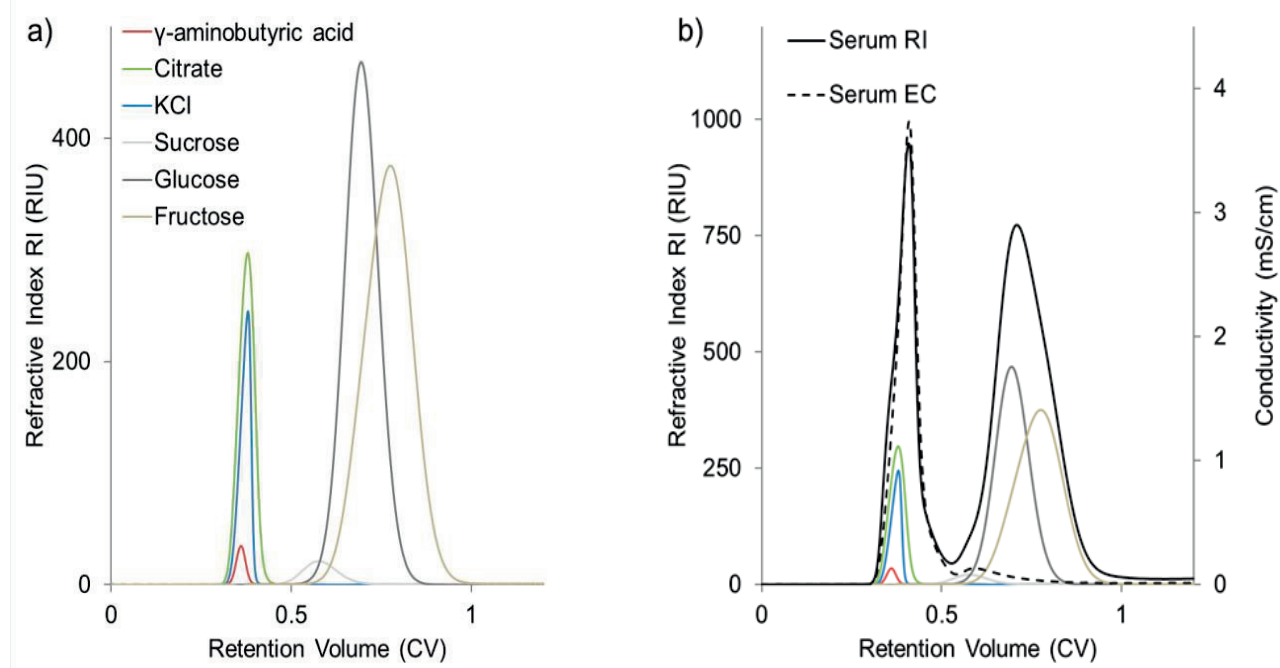

Fig. 5.2 - a) Overlay of eluted peaks from pulse injections of the key components of tomato serum detected via $R I$. b) Overlay of the same key components but with the additional overlay of diluted tomato serum detected with $R I$ and EC.

The viscosity of the tomato serum differed from the viscosity of the pure fructose and/or glucose mixtures. Fig. 5.3 shows the viscosity measured in tomato serum as a function of total sugar concentration (sum of fructose and glucose). The viscosity was much larger than data based on literature for either monosaccharide [14]. This is an indication for the presence of a, so far, unidentified molecule, that influences viscosity. This unknown contribution to viscosity, made the description of concentration and viscosity profiles inside the $S M B$ system inaccurate, as we will show later.

The $S M B$ process was designed to fractionate the feed stream into an enriched $\gamma$-aminobutyric acid fraction (low affinity, raffinate port) and saccharide fraction (high affinity, extract port) (see Fig. 5.1). To establish the equilibria of the separation, the isotherms were measured for the two major saccharide components (glucose and fructose) and minerals (as indicator for $\gamma$-aminobutyric acid) as function of feed viscosities $(1,2.5$, and $4 \mathrm{mPa} \cdot \mathrm{s})$. The affinity of fructose was slightly higher than of glucose, and with increasing viscosity, the affinity of both saccharides increased (Fig. 5.4a) and was linear in the range measured. The minerals isotherm was convex, and showed no discernable dependence on viscosity (Fig. 5.4b). One mineral isotherm was fitted over the whole concentration range and for the three measured viscosities (black dashed line in Fig. 5.4b). Due to the convex isotherm of the minerals, the selectivity between the sugars and minerals (as indicator for $\gamma$-aminobutyric acid) decreased at increased tomato serum concentration, which made the separation more difficult. 


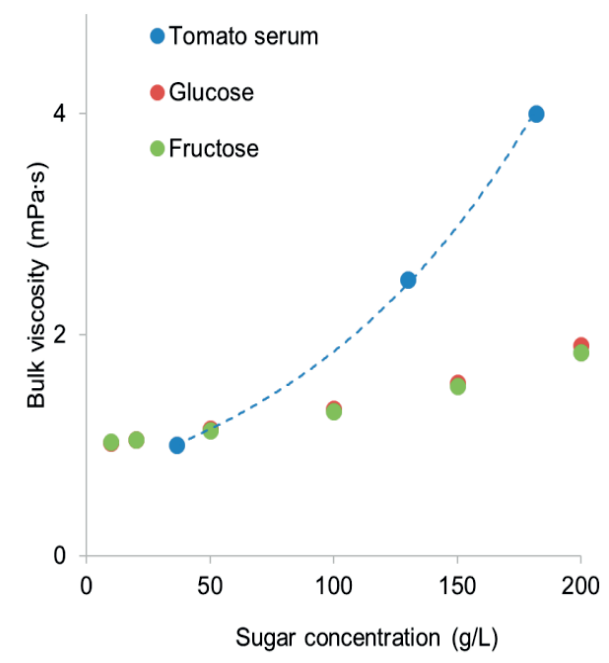

Fig. 5.3 - Difference in viscosity between tomato serum, glucose and sucrose solutions at equivalent concentrations.
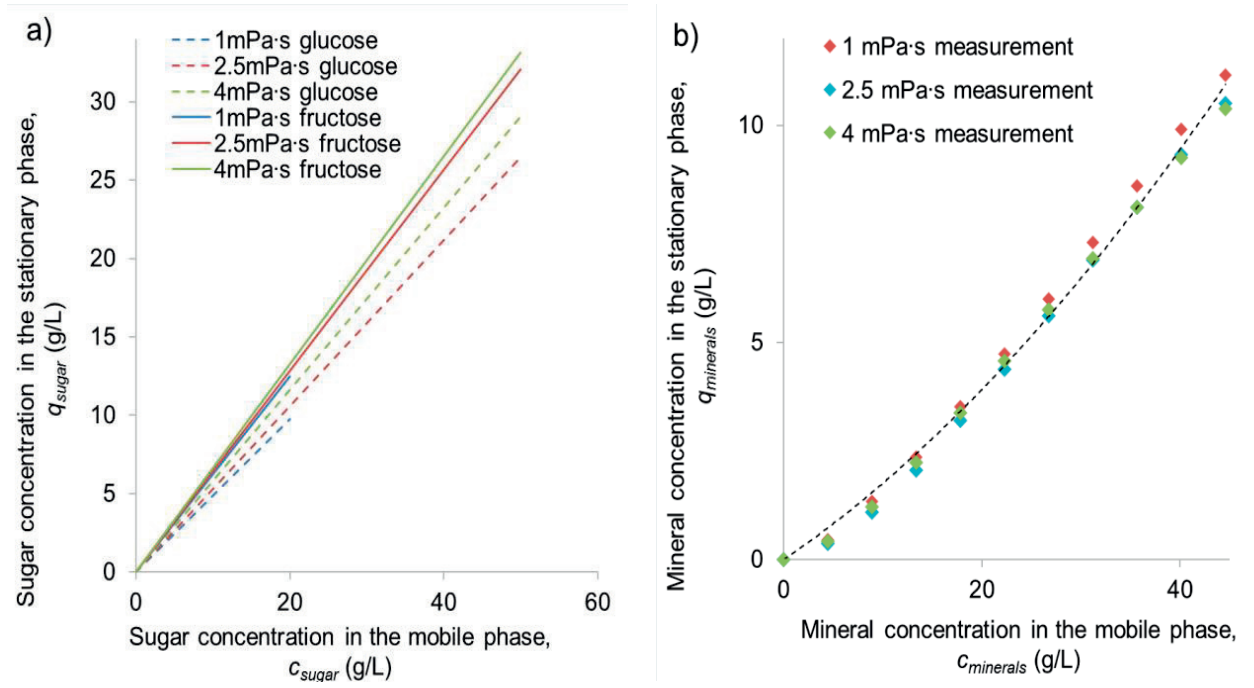

Fig. 5.4 - Isotherms of a) glucose and fructose and b) $\gamma$-aminobutyric acid in target bulk viscosities.

Based on the isotherms, the flow rates in the different sections were calculated with the triangle theory [12] for each viscosity. With the estimated flow rates and the kinetic parameters given in Table 5.3, flow rates and switching times were optimized (Table 5.4), in order to maximize productivity and minimize water input. 
Table 5.3 - Kinetic parameters calculated for the different viscosities.

\begin{tabular}{|c|c|c|c|c|}
\hline & Viscosity [mPa.s] & 1 & 2.5 & 4 \\
\hline \multirow{3}{*}{$k_{\text {overall }}^{\prime}[1 / s]$} & Fructose & 0.30 & 0.19 & 0.16 \\
\hline & Glucose & 0.30 & 0.19 & 0.16 \\
\hline & $\gamma$-Aminobutyric acid & 0.23 & 0.10 & 0.07 \\
\hline \multirow{3}{*}{$D_{L}\left[10^{-7} \cdot \mathrm{m}^{2} / \mathrm{s}\right]$} & Fructose & 6.96 & 2.80 & 1.75 \\
\hline & Glucose & 6.96 & 2.80 & 1.75 \\
\hline & $\gamma$-Aminobutyric acid & 6.20 & 2.09 & 1.17 \\
\hline
\end{tabular}

Table 5.4 - Optimized flow rates, calculated $\gamma$-aminobutyric acid recovery rates and sugar removal rates for optimized switch time (switch time 1) and switch times increased two times by roughly $25 \%(2 \& 3)$, for each viscosity.

\begin{tabular}{r|rrr} 
Feed viscosity [mPa·s] & $\mathbf{1}$ & $\mathbf{2 . 5}$ & $\mathbf{4}$ \\
\hline Feed [mL/min] & 9.74 & 6.42 & 5.44 \\
Extract [mL/min] & 16.34 & 10.56 & 7.93 \\
Raffinate [mL/min] & 13.97 & 10.71 & 9.56 \\
Water [mL/min] & 21.57 & 15.85 & 13.04 \\
Recycle [mL/min] & 11.37 & 10.62 & 8.07 \\
& & & \\
Switch time 1 [min] & 1.25 & 1.57 & 2.00 \\
Calculated $\gamma$-aminobutyric acid recovery [\%] & 99.4 & 96.0 & 93.9 \\
Calculated sugar removal [\%] & 99.8 & 9.3 & 99.1 \\
& & & \\
Switch time 2 [min] & 1.38 & 1.72 & 2.20 \\
Calculated $\gamma$-aminobutyric acid recovery [\%] & 100.0 & 100.0 & 100.0 \\
Calculated sugar removal [\%] & 90.7 & 89.0 & 88.0 \\
Switch time 3 [min] & 1.44 & 1.80 & 2.30 \\
Calculated $\gamma$-aminobutyric acid recovery [\%] & 99.6 & 99.8 & 99.8 \\
Calculated sugar removal [\%] & 85.4 & 81.1 & 76.7
\end{tabular}

\subsubsection{Pilot results}

Laboratory results often require slight adjustments of switching times from the calculated optima, a lesson learned through practical experience. Therefore the experiments were repeated twice for each viscosity, each time increasing the switching time by approximately $25 \%$ (Table 5.4). For both process criteria, the saccharide removal and $\gamma$-aminobutyric acid recovery, and for all three switching times, the experimental results are shown in Fig. 5.5. For the third switching time at viscosities of 2.5 and $4 \mathrm{mPa} \cdot \mathrm{s} \gamma$-aminobutyric acid recovery was not measured. The figure shows that the recovery of $\gamma$-aminobutyric acid was dependent on the feed viscosity, the results varying from about $55 \%(1 \mathrm{mPa} \cdot \mathrm{s}$, switch time 1$)$ to about $80 \%(4 \mathrm{mPa} \cdot \mathrm{s}$, switch time 2). The removal of sugar was decreasing at higher feed viscosities. Further, the trade-off between $\gamma$ aminobutyric acid recovery and sugar removal is visible. With increasing switch time the sugar removal decreases as the $\gamma$-aminobutyric acid recovery increases. 


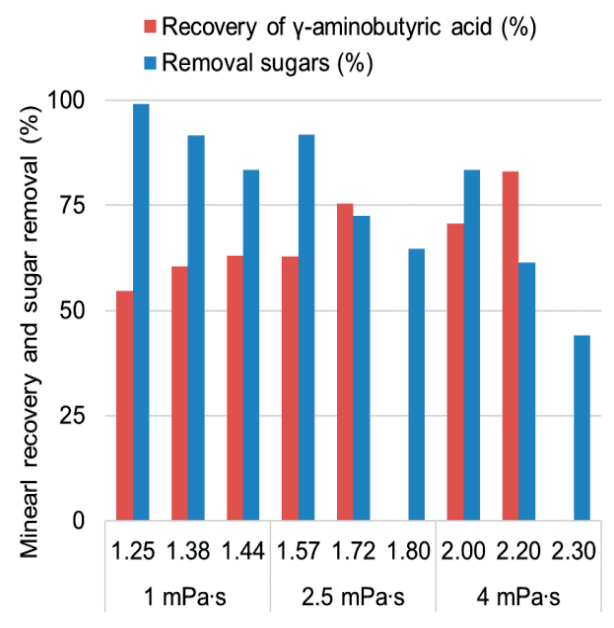

Switchin times ( $\mathrm{min})$ per feed viscosity

Fig. $5.5-\gamma$-Aminobutyric acid recovery and saccharide removal for three different viscosities measured from pilot scale experiments. Switching times were increased twice within each experimental series. No data for $\gamma$-aminobutyric acid recovery at the longest switching time in 2.5 and $4 \mathrm{mPa} \cdot \mathrm{s}$.

The separation process, resulted in enriched fractions of $\gamma$-aminobutyric acid. However, neither the target concentration of $\gamma$-aminobutyric acid, nor the targeted sugar removal reached the levels the process was designed for. With the exception of sugar removal at $1 \mathrm{mPa} \cdot \mathrm{s}$ (switch time 1), none of the values fell in the calculated range. To find an explanation for the discrepancy between the measured values and model, the distribution of viscosity within the system was analyzed.

\subsubsection{Viscosity distribution inside the $S M B$}

In the $S M B$ design a constant viscosity, equal to the feed concentration, was assumed. Fig. 5.6 shows the viscosity profile, measured via pressure drop, for each column in the $S M B$. At low viscosities (1 $\mathrm{mPa} \cdot \mathrm{s})$ the effect of dilution was not visible. However, at higher feed viscosity (2.5 and $4 \mathrm{mPa} \cdot \mathrm{s}$ ) dilution was observed and viscosity clearly varied between the columns. Fig. 5.6 shows good agreement with the expected relative distribution of viscosity throughout the system: the highest viscosities were measured in column 3 and 4, downstream and upstream of the feed port respectively. Moreover, Fig. 5.6 also shows that for feed with elevated viscosity, nowhere in the system the feed viscosity was measured, even at the highest viscosity in column three, the measured viscosity was about two-thirds of the feed viscosity $(1.7 \mathrm{mPa} \cdot \mathrm{s}$ for $2.5 \mathrm{mPa} \cdot \mathrm{s}$ feed and $2.7 \mathrm{mPa} \cdot \mathrm{s}$ for $4 \mathrm{mPa} \cdot \mathrm{s}$ feed). In the model input for the design of the pilot experiments, all parameters were based on the isotherm and mass transfer kinetics measurements at the feed viscosity. In the multicolumn separation, the input feed was diluted with the desorbent stream (water) and this should be taken into account. 


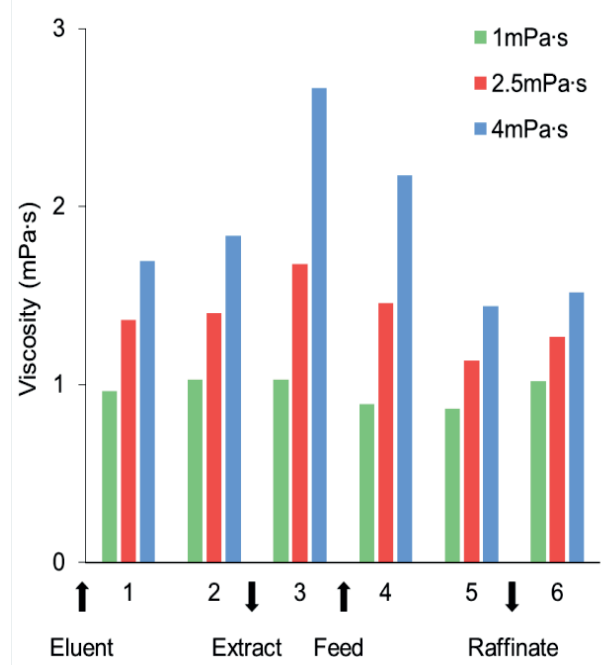

Fig. 5.6 - Viscosity for each column within the system and the feed viscosities of $1,2.5$, and $4 \mathrm{mPa} \cdot \mathrm{s}$. For orientation, the ports of eluent, extract, feed, and raffinate are marked between the columns.

Since the viscosity was reduced by dilution with water, the ratio of feed to eluent flow rate was used as dilution factor to estimate the average viscosity inside the $S M B$ system. From the dilution factor the total sugar concentration was calculated (given in Table 5.5). The total sugar concentration in $\mathrm{g} / \mathrm{L}$ is proportional to ${ }^{\circ} \mathrm{Bx}$. From the plot of viscosity as function of sugar concentration (Fig. 5.7), the viscosity of the diluted feed was fitted. The estimated viscosities were 1.2 and $1.5 \mathrm{mPa} \cdot \mathrm{s}$ for 2.5 and $4 \mathrm{mPa} \cdot \mathrm{s}$ feed, respectively. These were slightly lower than the measured values via pressure drop (1.4 and $1.9 \mathrm{mPa} \cdot \mathrm{s}$, respectively). The isotherms (Fig. 5.7b) and mass transfer parameters (Table 5.6) were re-evaluated for these average viscosities based on concentration. The isotherm for the minerals (as indicator for $\gamma$-aminobutyric acid) was independent of viscosity and was not changed (Fig. 5.4b). The change in mass transfer kinetics $k_{\text {overall }}$ and axial diffusivity $D_{L}$ were also estimated based on viscosity (Table 5.6). Using these re-evaluated equilibrium and kinetic parameters, the performances of the pilot experiments were calculated in the model.

Table 5.5 - Average viscosities within the $S M B$ system, as measured via pressure drop and estimated from dilution, based on ratio of eluent over feed flow. All viscosities in $(\mathrm{mPa} \cdot \mathrm{s})$.

\begin{tabular}{r|rrr} 
Feed viscosity $(\mathrm{mPa} \cdot \mathrm{s})$ & $\mathbf{1}$ & $\mathbf{2 . 5}$ & $\mathbf{4}$ \\
\hline Average viscosity measured via pressure drop $(\mathrm{mPa} \cdot \mathrm{s})$ & 1 & 1.4 & 1.9 \\
Ratio of flowrate eluent over feed $\left(q_{\text {eluent }} / q_{\text {feed }}\right)(-)$ & 2.2 & 2.5 & 2.4 \\
Total sugar concentration after dilution $(\mathrm{g} / \mathrm{kg})$ & 16 & 53 & 76 \\
Average viscosity based on dilution $(\mathrm{mPa} \cdot \mathrm{s})$ & 1 & 1.2 & 1.5
\end{tabular}



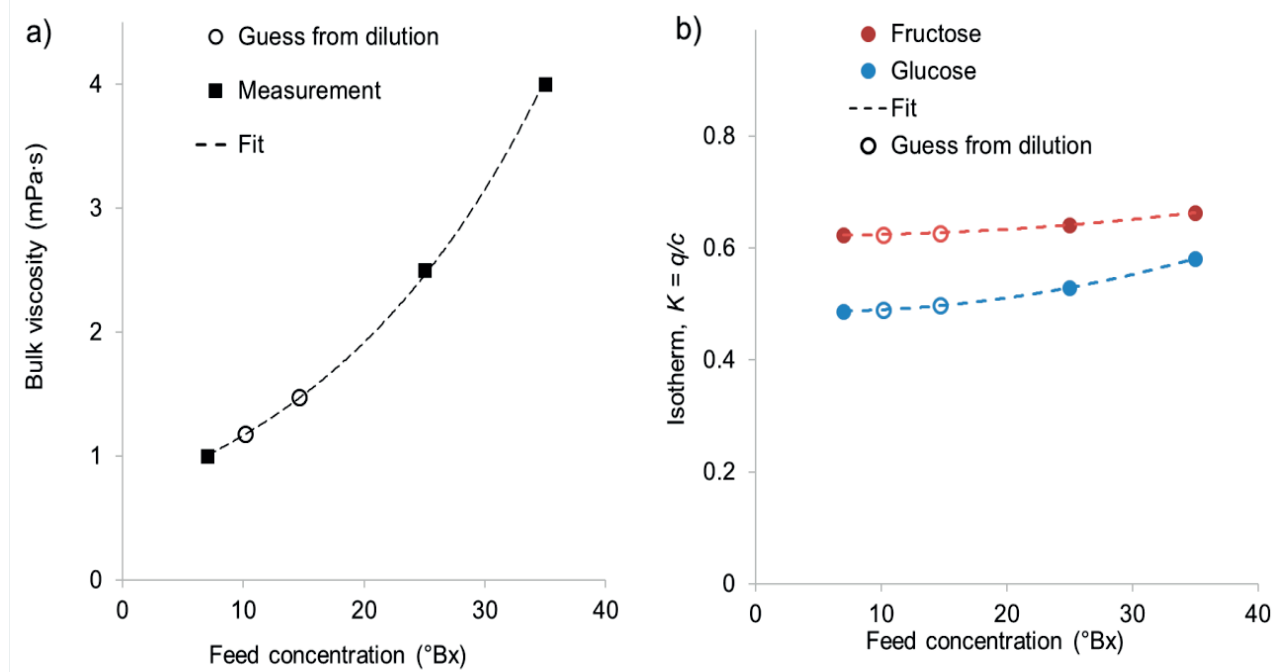

Fig. 5.7 a) Estimation of viscosities from dilution of feed concentration (open circles) over the system and measured viscosities (closed squares) and b) fitting of isotherm slopes for saccharides.
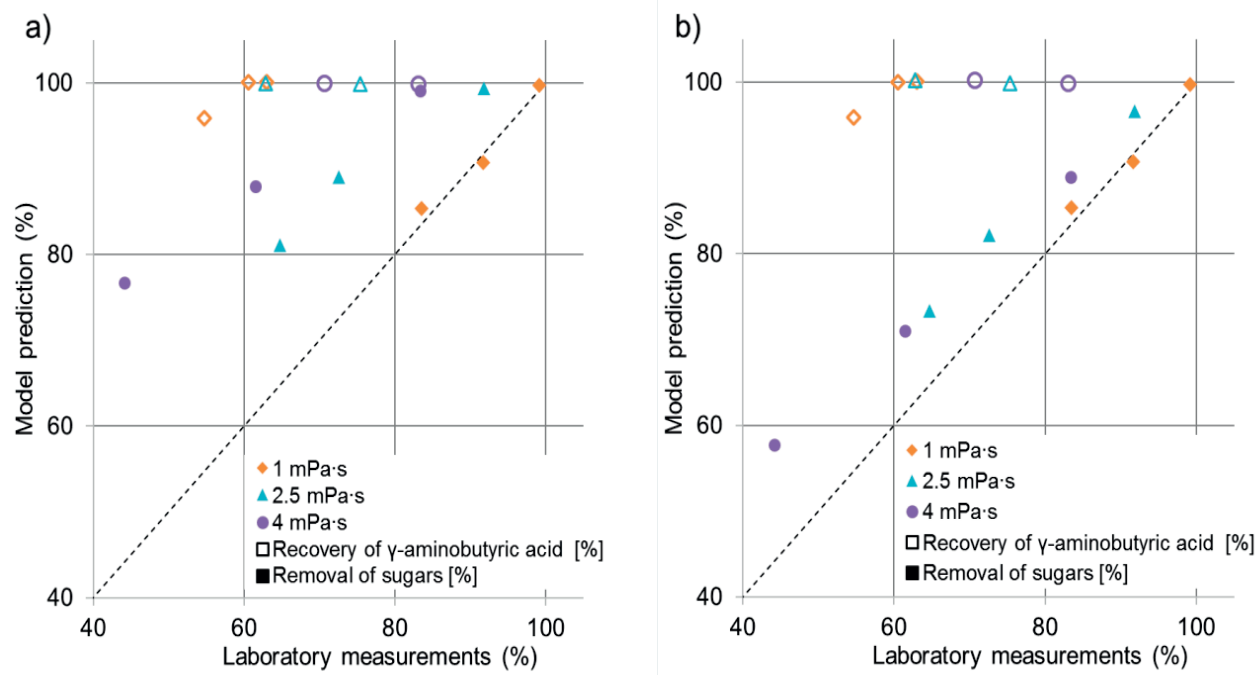

Fig. 5.8 - Plot comparing model calculation to experimental results from the laboratory pilot system for $\gamma$ aminobutyric acid recovery and saccharide removal for three different viscosities and three switching times, a) input parameters based on feed viscosity, b) input parameter based on viscosity of feed diluted by eluent. 
Table 5.6 - Kinetic parameters calculated for the different viscosities based on dilution of feed stream.

\begin{tabular}{|c|c|c|c|c|}
\hline & eed viscosity [mPa $\cdot \mathrm{s}]$ & 1 & 2.5 & 4 \\
\hline \multicolumn{2}{|c|}{ Average viscosity based on dilution [mPa.s] } & 1 & 1.2 & 1.5 \\
\hline \multirow{3}{*}{$k_{\text {overall }}^{\prime}[1 / s]$} & fructose & 0.30 & 0.28 & 0.26 \\
\hline & glucose & 0.30 & 0.28 & 0.26 \\
\hline & $\gamma$-aminobutyric acid & 0.23 & 0.21 & 0.18 \\
\hline \multirow{3}{*}{$D_{L}\left[10^{-7} \cdot \mathrm{m}^{2} / \mathrm{s}\right]$} & fructose & 6.96 & 6.31 & 5.31 \\
\hline & glucose & 6.96 & 6.31 & 5.31 \\
\hline & $\gamma$-aminobutyric acid & 6.20 & 5.55 & 4.56 \\
\hline
\end{tabular}

Fig. 5.8a shows the comparison of laboratory pilot measurements versus model calculations, which were based on equilibrium and kinetic parameters determined for a constant viscosity, equal to the feed viscosity, in the $S M B$ system. It is clear, that only the pilot data of sugar removal for $1 \mathrm{mPa} \cdot \mathrm{s}$ feed concentration match the calculation; sugar removal of at higher viscosities and all $\gamma$-aminobutyric acid recoveries showed poor agreement. The $\gamma$-aminobutyric acid recovery was always calculated to be close to $100 \%$, independent of viscosity and switching time.

Fig. 5.8b shows the calculation with the re-evaluated model parameters using the average viscosity based on the feed and eluent viscosity. The model calculations were repeated. The calculation of $1 \mathrm{mPa} \cdot \mathrm{s}$ feed concentration was the same. For both greater feed concentrations, it became apparent, that the calculated sugar removal was much closer to experimental values, even though the model overestimated sugar removal by roughly $10 \%$. The change in saccharide removal could be attributed to the change in isotherms, the influence of the changed kinetic parameters was small. Also the influence of switching time, is well represented in the model. The calculation of $\gamma$-aminobutyric acid recovery still requires improvement. It appears that the elution behavior of the mineral fraction is sensitive to concentration profiles within the system. In Fig. 5.3 it was shown that viscosities of tomato serum and model solution were not in agreement, possibly due to an unknown molecule. The change in viscosity and its influence on thermodynamics and kinetics needs to be identified and understood.

Literature described that mineral isotherms are dependent on sugar concentration [15]. At greater sugar concentration the capacity of the cation exchange resin for the minerals decreased. This could explain why a lower $\gamma$-aminobutyric acid recovery was observed at higher feed viscosities. Furthermore, during the pilot experiments, it was observed that divalent cations present in the tomato serum (such as $\mathrm{Ca}$ and $\mathrm{Mg}$ ) were exchanged with the ions on the cation exchange resin (such as $\mathrm{Na}$ and $\mathrm{K}$ ). It is known that the affinity of sugars for the cation exchange resin depends on the counter ion, and this may further have reflected on the mineral isotherms [16].

Additionally, viscous fingering, an instability at the interfaces between viscous sample and eluent, may have influenced the separation, at the two larger viscosities ( 2.5 and $4 \mathrm{mPa} \cdot \mathrm{s}$ ). The result of viscous fingering is an instable interface, leading to distorted peaks and therefore reduced separation performance [17]. It occurs where a low viscous liquid displaces a high viscous liquid. In the $S M B$ operated at higher feed viscosities, this happens where the eluent enters the system and it therefore should have little influence on the recovery of $\gamma$ aminobutyric acid. 


\subsubsection{Water use and productivity as function of feed input viscosity}

The use of water and the productivity of the chromatographic system for different input stream viscosities were compared to evaluate the water saving potential and resin volume reduction of chromatography operated at higher viscosities. The comparison was made for optimized systems, minimal water input for eluent and maximum productivity, using the re-evaluated parameters for the average viscosity within the $S M B$. As the model overestimated $\gamma$-aminobutyric acid recovery compared to measurements in the pilot system, the results may be optimistic, but still serve the purpose of comparison. Water use for dilution of the concentrated tomato serum to the viscosities of the input stream was included in the total water use.

The recovered mass of $\gamma$-aminobutyric acid, by lieu of recovered mineral fraction, was compared to the use of water for eluent and feed dilution calculated in the model (Fig. 5.9a). The comparison clearly showed that operating at higher viscosities $(2.5-4 \mathrm{mPa} \cdot \mathrm{s})$ used much less water per $\mathrm{kg}$ of recovered target fraction and resulted in a higher productivity (mass of recovered product per system volume and time, Fig. 5.9b). This result matched previously calculated column performances in single column experiments (chapter 4), where it was found that the trade-off between mass transfer and stream volume which was made when changing viscosity, lead to an increase in column volume in feed streams with viscosities below about $2.5 \mathrm{mPa} \cdot \mathrm{s}$.
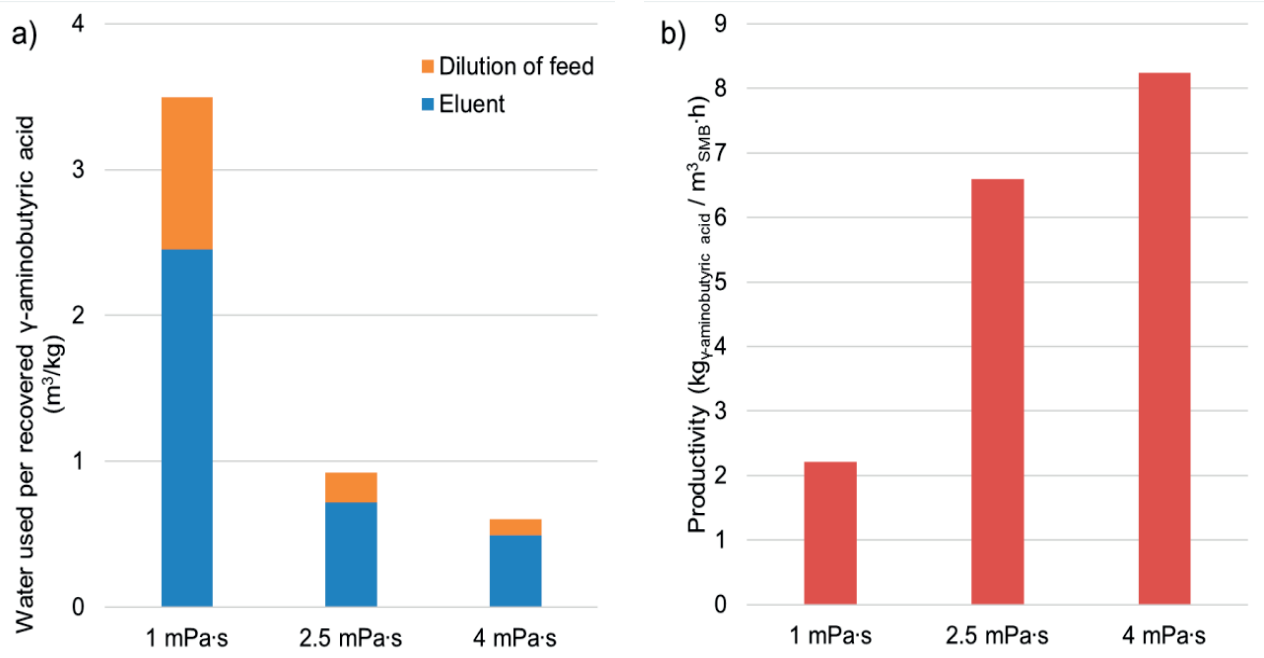

Fig. 5.9 - After optimization of velocities in each section of the $S M B$, a) water use per kg of recovered $\gamma$-aminobutyric acid for three feed viscosities for dilution of feed and for eluent use and $b$ ) productivity for three feed viscosities were calculated.

\subsection{Conclusions}

The footprint of an $S M B$ system can be improved, by optimizing feed stream viscosity. Whether dilute streams are concentrated, or concentrated streams are diluted, a distinct change in productivity and water use is found in between viscosities of 1 and $2.5 \mathrm{mPa} \cdot \mathrm{s}$, the higher viscosity outperforming lower viscosity. Productivity is 
increased by a factor of around 3 and water use is reduced by around the same factor. The difference between 2.5 and $4 \mathrm{mPa} \cdot \mathrm{s}$ is less pronounced, both in terms of productivity and water use. Within an $S M B$ operated at higher feed concentrations, concentration dependent parameters can be estimated based on the dilution of the feed with the eluent. In this manner the measured removal of sugars from tomato serum using ion-exclusion chromatography matches design calculations well. The measured recovery of $\gamma$-aminobutyric acid enriched fraction, is not in agreement with the model design. The behavior of $\gamma$-aminobutyric acid in such a system in very sensitive to concentration differences due to convex isotherms, sugar affinities that depend on the counter ion, and the interaction between the saccharides and the minerals, requiring the measurements of multicomponent isotherms.

\subsection{Acknowledgements}

This research took place within the framework of the Institute for Sustainable Process Technology ISPT. The authors would like to thank the ISPT for their support, together with Unilever (Vlaardingen, NL), FrieslandCampina Research (Amersfoort, NL), DSM (Delft, NL) and Cosun Food Technology (Roosendaal, $\mathrm{NL}$ ) for their financial support and interest in this project.

\subsection{References}

1. A.J.v.d. Goot, P.J.M. Pelgrom, J.A.M. Berghout, M.E.J. Geerts, L. Jankowiak, N.A. Hardt, J. Keijer, M.A.I. Schutyser, C.V. Nikiforidis, R.M. Boom, Concepts for further sustainable production of foods, Journal of Food Engineering 168 (2016), (2016).

2. H. Schmidt-Traub, M. Schulte, A. Seidel-Morgenstern, Preparative Chromatography, Wiley, 2012.

3. Dow, Ion exchange and adsorbent solutions for the nutrition market, in https://www.dow.com/webapps/include/GetDoc.aspx?filepath=liquidseps/pdfs/noreg/17703529.pdf, 2017.

4. M. Verzele, Industrial application of preparative liquid chromatography, TrAC Trends in Analytical Chemistry, 6 (1987) 202-205.

5. A. Schultze-Jena, M.A. Boon, R.C. Vroon, P.J.T. Bussmann, A.E.M. Janssen, A. van der Padt, High viscosity preparative chromatography for food applications, in, to be published, 2019.

6. J.A. Vente, H. Bosch, A.B. de Haan, P.J.T. Bussmann, Evaluation of sugar sorption isotherm measurement by frontal analysis under industrial processing conditions, Journal of Chromatography A, 1066 (2005) 71-79.

7. A. Felinger, G. Guiochon, Comparison of the Kinetic Models of Linear Chromatography, Chromatographia, 60 (2004) S175-S180.

8. A. Schultze-Jena, M.A. Boon, P.J.T. Bussmann, A.E.M. Janssen, A. van der Padt, The counterintuitive role of extra-column volume in the determination of column efficiency and scaling of chromatographic processes, Journal of Chromatography A, 1493 (2017) 49-56.

9. F. Gritti, G. Guiochon, Mass transfer kinetics, band broadening and column efficiency, Journal of Chromatography A, 1221 (2012) 2-40.

10. B. Coquebert de Neuville, A. Tarafder, M. Morbidelli, Distributed pore model for bio-molecule chromatography, Journal of Chromatography A, 1298 (2013) 26-34. 
11. E.J. Wilson, C.J. Geankoplis, Liquid Mass Transfer at Very Low Reynolds Numbers in Packed Beds, Industrial \& Engineering Chemistry Fundamentals, 5 (1966) 9-14.

12. G. Carta, A. Jungbauer, Design of Chromatographic Processes, Protein Chromatography, Wiley-VCH Verlag GmbH \& Co. KGaA, 2010, pp. 309-339.

13. S. Ergun, A.A. Orning, Fluid Flow through Randomly Packed Columns and Fluidized Beds, Industrial \& Engineering Chemistry, 41 (1949) 1179-1184.

14. Nordic Sugar A/S, The functional properties of sugar - on a technical level, in www.nordicsugar.com.

15. M.-L. Lameloise, R. Lewandowski, Purification of beet molasses by ion-exclusion chromatography: fixed-bed modelling, Journal of Chromatography A, 685 (1994) 45-52.

16. J. Vente, H. Bosch, A. De Haan, P. Bussmann, Comparison Of Sorption Isotherms Of Mono- and Disaccharides Relevant To Oligosaccharide Separations For Na, K, And Ca Loaded Cation Exchange Resins, Chemical Engineering Communications, 192 (2005) 23-33.

17. H.J. Catchpoole, R. Andrew Shalliker, G.R. Dennis, G. Guiochon, Visualising the onset of viscous fingering in chromatography columns, Journal of Chromatography A, 1117 (2006) 137-145. 
Chapter 6

General discussion 


\subsection{Main findings and conclusions}

This thesis aims at understanding the interplay between feed viscosity, mass transfer resistance, pressure drop and eventually productivity and water use of a chromatographic system. With the aim of defining a window of operation which includes feed concentration as design parameter. Chapter 2 addresses method development for measuring mass transfer resistance in a chromatographic column in such a way that the data can be used to scale up to industrial scale. It was shown that correction for extra-column contribution to band broadening must be made in many cases, otherwise column efficiency is overestimated and subsequently industrial designs under-dimensioned.

With the right mass transfer measurement in place, chapter $\mathbf{3}$ focuses on the possibility to predict the parameter describing the mass transfer inside the stationary phase: the intraparticle diffusivity $D_{p}$. Mass transfer inside the stationary phase is often the rate determining step in industrial chromatographic processes, because these are operated with relatively large particle diameters to accommodate large throughput. Stationary phase properties, relevant to intraparticle diffusion, such as pore size, tortuosity, and particle porosity, were determined and related to intraparticle diffusivity using existing models. For SEC it was shown that by adapting the Mackie and Mears model, using accessible pore volume fraction instead of particle porosity alone, intraparticle diffusivity can be predicted for different stationary phases and diffusing molecules.

Having more understanding on intraparticle diffusivity, chapter $\mathbf{4}$ expands this knowledge towards the use of highly viscous feed streams. The dependence of intraparticle diffusivity on the viscosifiers ability, those molecules in the mobile phase which contribute to viscosity, to penetrate pore volume was shown. The ability to penetrate pore volume is expressed by the partition coefficient $K_{D}$. These results were used to determine the window of operation for viscous feed streams. It was shown that diluting highly viscous feed streams prior to chromatographic separation, should go no further than approximately $2.5 \mathrm{mPa} \cdot \mathrm{s}$. If the feed stream is diluted to lower viscosities, column volume will increase. Diluting feed streams from $8 \mathrm{mPa} \cdot \mathrm{s}$ down to around $2.5 \mathrm{mPa} \cdot \mathrm{s}$ showed little influence on column volume, but column dimensions changed, with a tendency to get narrower but longer as viscosity decreases.

Finally, in chapter 5, the knowledge gained from working at high viscosities in single column systems is challenged on a multicolumn simulated moving bed $S M B$ system with tomato serum as feed and ion-exclusion as separation mechanism. The aim was obtaining a $\gamma$-aminobutyric acid enriched and monomeric sugar strapped fraction. The behavior of the sugars for different feed viscosities is calculated well by the model when the ratio of feed to eluent is used as dilution factor. The behavior of $\gamma$-aminobutyric acid in ion-exclusion chromatography is highly concentration dependent and the recovery could not be calculated with accuracy. The $S M B$ at the two higher feed viscosities (2.5 and $4 \mathrm{mPa} \cdot \mathrm{s}$ ) outperformed the $S M B$ at the lower feed viscosity $(1 \mathrm{mPa} \cdot \mathrm{s})$ both in terms of water use and productivity. 


\subsection{Using temperature to reduce viscosity}

In the interplay between feed viscosity, mass transfer resistance, pressure drop, and productivity of a chromatographic system, also other aspects require discussion, which have only been mentioned in brief at this point, e.g. the relationship between temperature and viscosity.

In practice, to reduce the viscosity of feed streams, separation processes are often run at elevated temperatures. The viscosity of liquids is inversely related to temperature, as Fig. 6.1 shows for three different sugar solutions at $20 \%(\mathrm{w} / \mathrm{w})$ concentration. For instance the desalting of high fructose corn syrup by ion-exchange chromatography is performed at temperatures around $60^{\circ} \mathrm{C}$ with a dry weight of $42 \%(\mathrm{~m} / \mathrm{m})$ [1]. The temperature increase reduces the viscosity from $7.4 \mathrm{mPa} \cdot \mathrm{s}$ at $20^{\circ} \mathrm{C}$ to $1.9 \mathrm{mPa} \cdot \mathrm{s}$ at $60^{\circ} \mathrm{C}$ (estimated assuming the viscosity equals a fructose solution).

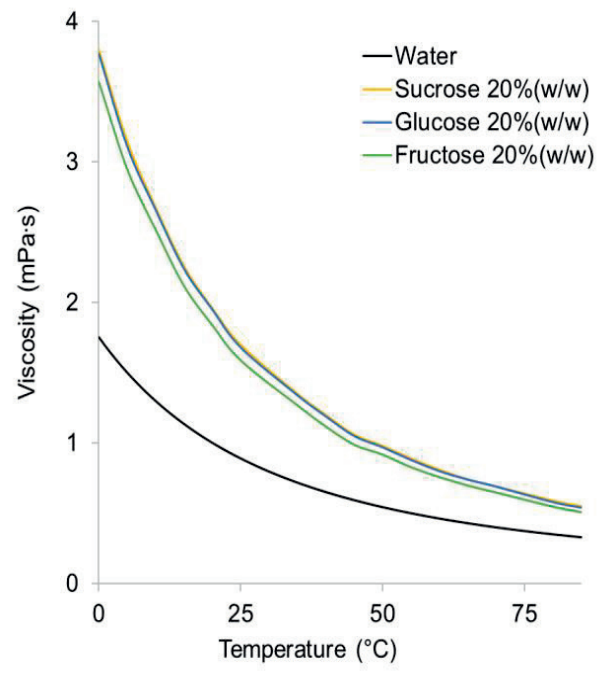

Fig. 6.1 - Viscosity as function of temperature for water and sugar solutions at $20 \%(\mathrm{w} / \mathrm{w})$. Water viscosity from [2] and sugar viscosities from [3].

In case the functionality of the target components inside the feed stream is not sensitive to temperature, increasing temperature to facilitate chromatography may well increase separation performance. Elevated temperatures enhance mass transfer by increasing diffusivity of molecules directly and by decreasing viscosity (equation 4.5). The viscosity reduction enables maintaining a relatively low pressure drop. However, temperature also influences the sorption isotherms and selectivity may decrease at elevated temperatures [4]. How the sorption isotherm is influenced by the temperature depends on the underlying thermodynamics. Another aspect of temperature is the possibility of microbial growth. Growth kinetics of many food contaminants favor intermediate temperatures, therefore processing temperatures below $5^{\circ} \mathrm{C}$ or above $60^{\circ} \mathrm{C}$ may have to be chosen.

The determination of optimum temperature in combination with elevated viscosities of the feed stream requires experiments measuring the influence of temperature on mass transfer, viscosity and sorption 
isotherms. As a first indication, van Deemter curves of $\mathrm{D}_{2} \mathrm{O}$, injected as pulses on a column packed with Dowex Monosphere $99 \mathrm{Ca} / 320$, were recorded at 5,25 , and $50^{\circ} \mathrm{C}$ in Milli-Q water as mobile phase. In the experimental setup, feed and column were temperature regulated and the tubing was isolated (for further details on the setup, see chapter 2). The data show, that with increase in temperature, the slope of van Deemter curves decrease and plate heights are reduced (Fig. 6.2).

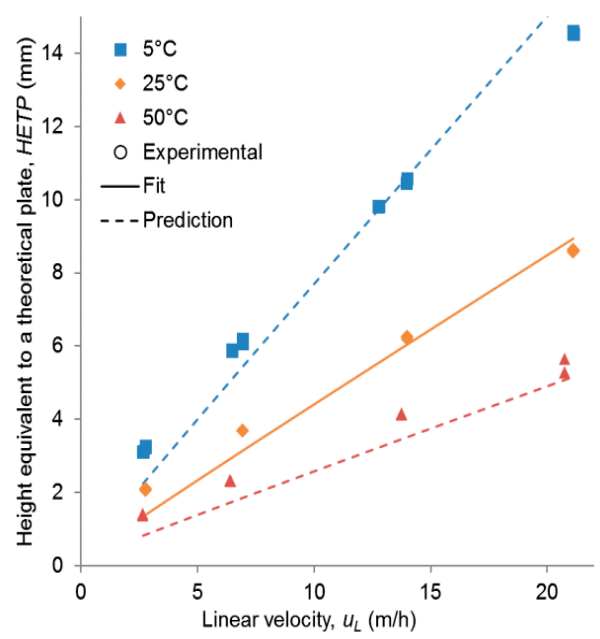

Fig. 6.2 - Van Deemter curves of $\mathrm{D}_{2} \mathrm{O}$ in Milli-Q water as function of temperature. Line at $25^{\circ} \mathrm{C}$ fitted, lines at 5 and $50^{\circ} \mathrm{C}$ predicted. Experimental set-up as described in chapter 2.

It is expected that the intraparticle diffusivity, which determines the slope of a van Deemter curve has the same temperature dependency as the bulk diffusion coefficient (equation 3.2) [5]. From measurements at $25^{\circ} \mathrm{C}$, the intraparticle diffusivity $D_{p}$ was estimated (equation $3.9-3.11$ ) and the bulk diffusion coefficient $D_{m}$ was calculated using the relationship of Wilke and Chang, both described in chapter 3. According to the parallel pore model $[6,7]$, the ratio of $D_{P} / D_{m}$ is temperature independent. The ratio of $D_{P} / D_{m}$ was used to calculate the van Deemter curve slope for 5 and $50^{\circ} \mathrm{C}$. Fig. 6.2 shows that the change in mass transfer resistance with temperature is quite accurately calculated.

It is concluded that the temperature influence on mass transfer kinetics is already well understood and can easily be implemented in mathematical models. To fully include temperature as a design parameter the influence on the sorption isotherms and the underlying thermodynamics also have to be understood.

\subsection{Requirement of constant number of plates for various feed viscosities}

Within this research the height equivalent to a theoretical plate HETP is used as indicator of the performance of chromatographic columns. The required number of theoretical plates $N$ determines, if a separation goal can be reached and combined with HETP it determines the size of the system (equations $1.1 \& 1.2$ ). The required number of plates is dependent on selectivity and peak width. For a single column and two symmetrical 
Gaussian peaks, the required number of plates $N$ to achieve a given resolution $R$, e.g. $R=1.5$ for complete separation, can be calculated using [4]:

$$
N=16 R^{2}\left(\frac{\alpha}{\alpha-1} \frac{k+1}{k}\right)^{2}
$$

With selectivity $\alpha$ as ratio of retention factors of the two components and $k$ the retention factor of the component with the longer retention time.

In case one or both of the peaks are asymmetrical, overlap of the two peaks can change. A basic separation example for the separation of two components eluting with different peak shapes is given in Fig. 6.3. The figure illustrates the separation of different peak shapes from a second component with a constant Gaussian peak shape. The deviation of a peak shape from a Gaussian peak is quantified with the tailing factor $T F$, which is calculated from the distance of the two flanks, at a certain height, from the peak maximum, $a$ and $b$ respectively, see equation 6.2 [4]. In this work the tailing factor is calculated from the points where the flanks had reached $5 \%$ of the maximum peak height.

$$
T F=\frac{a+b}{2 a}
$$

It is clear that the required number of plates for a given separation is dependent on peak shapes from the overlapping area in Fig. 6.3.

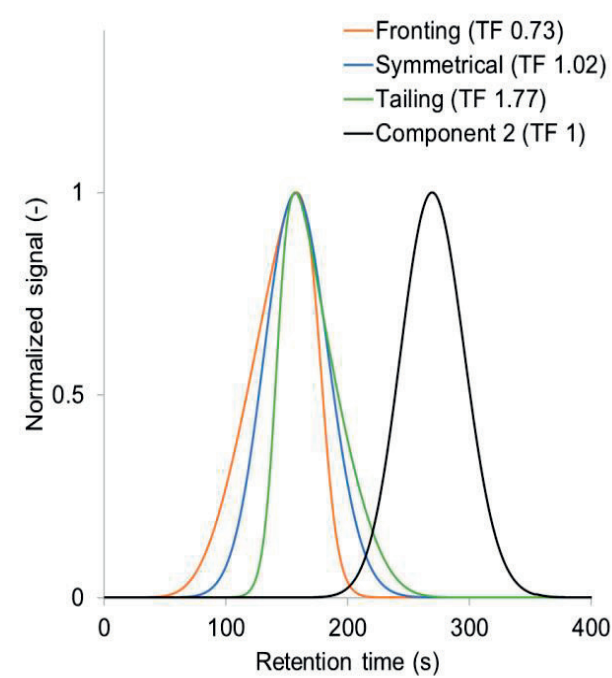

Fig. 6.3 - Change of separation behavior at constant plate number, but different peak symmetries.

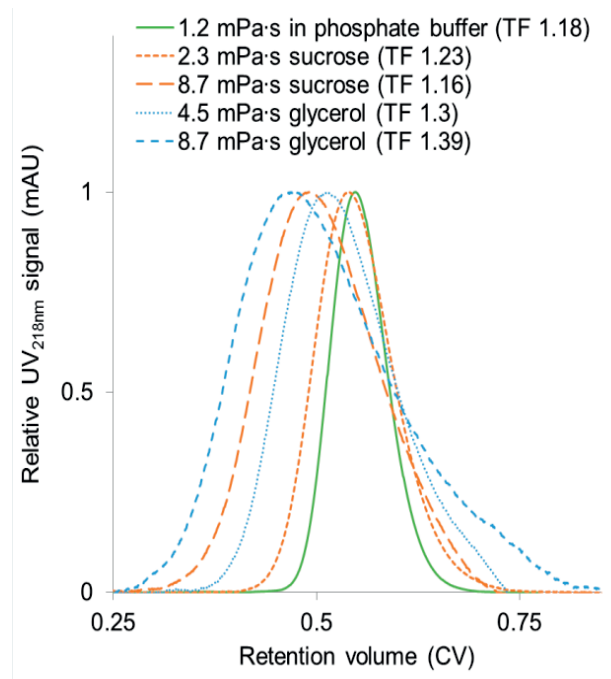

Fig. 6.4 - Overlaid chromatograms of triglycine in phosphate buffer, and mobile phase viscosified with sucrose at 2.3 and $8.7 \mathrm{mPa} \cdot \mathrm{s}$ and glycerol at 4.5 and $8.7 \mathrm{mPa} \cdot \mathrm{s}$ at $u_{S}=1 \mathrm{~m} / \mathrm{h}$.

Experiments with different mobile phases show that mobile phase viscosity affects the peak shape. Fig. 6.4 shows elution peaks of triglycine recorded at viscosities ranging from 1.2 to $8.7 \mathrm{mPa} \cdot \mathrm{s}$. 
With changing viscosity, peak symmetries change from $T F 1.16$ to 1.39. Further, the retention volume is reduced at elevated viscosities. Within this thesis, when comparing systems for various feed viscosities, $N$ (and the pressure drop) were kept constant. In chapter 5, the retention volume was taken into account via the slope of the isotherm. Changes in peak shape were not taken into account. However, this change due to viscosity should be considered. Process design requires knowledge on peak shapes in order to calculate operational parameters for the separation of two components. With changing viscosity, change in peak shape must be accounted for in the design. More insight is required on underlying thermodynamic phenomena, e.g. information on the isotherms, to enable the calculation of the required number of plates for a given separation challenge as function of feed viscosity.

\subsection{Future perspectives}

Food industry is increasingly aware of the potential value of minor components, which are available in large agro-feed streams but are thus far underutilized. The challenge in extracting minor components can be their low concentration, but also the complex mixture of similar components in the stream. Industrial chromatography, a still emerging technology in agro-food, could play an important role in this. However, chromatography on this scale is rather expensive both in investment and operational costs. One way to reduce these costs is by reducing system size which is realizable by concentrating the feed stream. This thesis shows that there are limits to this kind of size reduction due to viscosity phenomena, through experiments in singleand multicolumn systems. At viscosities greater than about $2.5 \mathrm{mPa} \cdot \mathrm{s}$, increases in pressure drop and reduction in mass transfer limit the benefits gained by feed stream volume reduction. One possible way to work around this issue is by improving characteristics of the stationary phase. Reducing the size of the stationary phase particles reduces the characteristic length molecules have to traverse by diffusion, which results in reduced hindrance to mass transfer inside the particle pores. With decreasing particle size, however, pressure drop over the column bed will increase as well. This in turn can be countered by the use of stationary phases that are open and allow for convection within the particles. Open particles can potentially change the link between particle size and pressure drop. Process designers should consider an increase of temperature to reduce viscosity, a common custom in processes of sugar syrup. With increase in temperature, stream viscosity can be reduced significantly, resulting in higher mass transfer and lower back pressure. However, if the components of the stream are temperature sensitive, increase in temperature may not be an option. Furthermore, in temperatures between around 25 and $40^{\circ} \mathrm{C}$, microbial growth may be an issue, necessitating processing at temperatures either high enough or low enough to limit microbial growth.

The behavior of components within a chromatographic column is determined by thermodynamics, mass transfer kinetics, and fluid dynamics. The focus of this thesis is on mass transfer kinetics. The fluid dynamics are taken into account by way of pressure drop. The thermodynamics are neglected, although influence of viscosity on isotherms (Fig. 5.4), peak shapes and peak positions (Fig. 6.4) are observed in some cases. Complex multicolumn separation processes will be better understood once the change in concentration profile within the system, and its influence on isotherm and mass transfer kinetics, is implemented into the design models. 


\subsection{Acknowledgements}

The authors wish to thank Sasja Antonisse for performing experiments and Melda Mutlu for making model calculations.

\subsection{References}

1. P. Corporation, Corn Sweetener Refining with Ion Exchange Resins, in: www.purolite.com, 2007.

2. I.A.P.W.S., Release on the IAPWS Formulation 2008 for the Viscosity of Ordinary Water Substance, in, IAPWS, http://www.iapws.org/relguide/visc.pdf, 2008, pp. 9.

3. V.R.N. Telis, J. Telis-Romero, H.B. Mazzotti, A.L. Gabas, Viscosity of Aqueous Carbohydrate Solutions at Different Temperatures and Concentrations, International Journal of Food Properties, 10 (2007) 185-195.

4. A. Seidel-Morgenstern, M. Schulte, A. Epping, H. Schmidt-Traub, M. Schulte, A. SeidelMorgenstern, Fundamentals and General Terminology, Preparative Chromatography, Wiley-VCH Verlag GmbH \& Co. KGaA, 2012, pp. 7-46.

5. F. Gritti, G. Guiochon, Mass transfer kinetics, band broadening and column efficiency, Journal of Chromatography A, 1221 (2012) 2-40.

6. C.N. Satterfield, Mass transfer in heterogeneous catalysis, M.I.T. Press, 1970.

7. M. Suzuki, Adsorption engineering, Kodansha, 1990. 

Summary 
Agro-food streams, present in large volumes, contain compounds attractive for food industry, if they are separated from unwanted components. Recovery of such components offers a large potential for industrial applications. A trend towards enriched fractions, rather than purified products, enables sustainable process design via chromatographic separation. Chromatography has the potential to fractionate these agro-food streams at large industrial scale, while maintaining functionality and operating in a sustainable and economical manner. The separation mechanism can be fine-tuned to target specific molecular characteristics. Because the streams to be processed are so large, large equipment is required, which renders the process economically unfeasible. Process economics can be improved by reducing the size of the chromatographic installation, which is directly dependent on the volume to be processed. The stream to be processed can be reduced by increase of concentration, which leads to higher viscosity. The aim of this thesis is to understand the interplay between feed viscosity, mass transfer resistance, pressure drop and eventually productivity and water use of a chromatographic system.

In the design of industrial chromatographic processes, both experiments at lab scale and model calculations are used. Experiments are performed to determine model parameters and to validate the model. Subsequently the model is used to scale-up. Experiments require, in addition to the column, the use of further equipment (connectors, tubing, valves, detectors, etc.), all of which add to extra-column volume. The contribution of the extra-column volume to peak broadening is often neglected assuming that by doing so the column efficiency is underestimated and scaling up results in an oversized system. Chapter 2 addresses method development for measuring the mass transfer resistance in a chromatographic column in such a way that the data can be used to scale up to industrial scale. It was shown, that correction for the extra-column contribution to band broadening must be made in many cases, otherwise the column efficiency is overestimated and subsequently industrial designs under-dimensioned.

In chapter 3, the main hindrance to mass transfer is identified and measured: the intraparticle diffusivity. In industrial applications, generally stationary phase particles of large diameters are used. Large particle sizes facilitate operation at a low pressure drop, but they introduce large characteristic lengths that the molecules have to travers by diffusion. It is difficult to predict intraparticle diffusivity and therefore measurement for each new molecule/stationary phase combination is required. In an attempt to link and quantify pore and molecule characteristics, intraparticle diffusivities, pore diameters, porosities, and tortuosities are measured in various stationary phases with a selection of (relatively) small molecules. Two models from literature that relate stationary phase properties to intraparticle diffusivity, are tested for their predictive quality, but give unsatisfactory results. By modifying one of the models with the addition of the accessible pore fraction, calculated from inverse size exclusion measurements, a good fit of all intraparticle diffusivities for all stationary phases and all molecules is observed. This improved model can serve as a predictive tool for intraparticle diffusivity.

Implications of using a concentrated feed with an increased viscosity on mass transfer resistance inside a chromatographic column is described in chapter 4. Dependence of intraparticle diffusivity on viscosifiers ability, those molecules in the mobile phase which contribute to viscosity, to penetrate pore volume is shown. The ability to penetrate pore volume is expressed by the partition coefficient $K_{D}$. These results are used to determine the window of operation for viscous feed streams. It is shown that diluting highly viscous feed streams prior to chromatographic separation, should go no further than approximately $2.5 \mathrm{mPa} \cdot \mathrm{s}$. If the feed stream is diluted to lower viscosities, column volume will increase. Diluting feed streams from $8 \mathrm{mPa} \cdot \mathrm{s}$ down 
to around $2.5 \mathrm{mPa} \cdot \mathrm{s}$ shows little influence on column volume, but column dimensions change, with a tendency to get narrower and longer as viscosity decreases.

In chapter 5, knowledge gained from working at high viscosities in single column systems is challenged on a multicolumn simulated moving bed $S M B$ system with tomato serum as feed and ion-exclusion as separation mechanism. The aim was obtaining a $\gamma$-aminobutyric acid enriched and monomeric sugar strapped fraction. The behavior of the sugars for different feed viscosities is calculated well by the model when the ratio of feed to eluent is used as dilution factor. The behavior of $\gamma$-aminobutyric acid in ion-exclusion chromatography is highly concentration dependent and the recovery is not calculated with accuracy. The $S M B$ at the two higher feed viscosities (2.5 and $4 \mathrm{mPa} \cdot \mathrm{s}$ ) outperforms the $S M B$ at the lower feed viscosity ( $1 \mathrm{mPa} \cdot \mathrm{s}$ ) both in terms of water use and productivity.

Finally in chapter 6 the main findings and conclusions are discussed. Further addressed are the use of temperature to reduce viscosity and an assumption used in this thesis, that the required number of theoretical plates remains constant when comparing designs for various feed concentrations. At the end of this chapter an outlook is given on future perspectives for the effort of decreasing chromatographic system size. 



\section{Acknowledgements}

I did write this thesis, but I did not work on it by myself. Along the four years as $\mathrm{PhD}$ student, I have met and worked with a number of people. Many have had an influence on this work and on myself, and I am grateful for each contribution, big or small.

No doubt the greatest influence had my supervisor Floor Boon, to whom I am deeply thankful. Floor, I want to thank you for the countless discussions, little meetings, big meetings, your explanations, perspective and patience when you explained something again. I truly enjoyed working with you during my time as $\mathrm{PhD}$ student and am happy that we continue working together. Next I want to thank Anja Janssen, my supervisor from the University chair group. Thank you for all your advice, in science, in academia, and how to help a baby sleep through the night. Your motivation meant a lot to me. My promotor, Albert van der Padt, thank you for your keen observations and the time you took for me. Your motivational influence was greater than you might think and I thank you very much for supporting me throughout this time and of course for the opportunity to write this thesis in the first place. Also I really appreciated our visit to the FrieslandCampina plant in Borculo. Paul Bussmann, thank you for the many conversations, discussions, and arguments with all your professional insight. Ronald Vroon, the wisdom and knowledge I found in you could fill books. Thank you for all the help in setting up experiments, helping out the thesis students, and discussing results.

Thank you Juliana Romero Guzman and Pina Fritz, for being my paranymphs at the day of my defense. Thank you Bijoy for organizing the weekly football matches and adding your perspective to the Dutch language. Eet smakie Bijoy, eet smakie.

I want to thank my colleagues from TNO and WUR-FBR, especially Peter Geerdink and Corjan van den Berg. Jan Jetten, group manager of Functional Ingredients at TNO and later of Biorefinery and Sustainable Value Chains at FBR, thank you for giving me the opportunity to work in your group.

Although I spent far less time at my university chair group, Food Process Engineering, than at TNO/FBR, the group has had a great influence on me and my work, that I want to acknowledge next. Remko Boom, you are leading an extraordinary group, filled with contagious energy and great people. From the weekly Thursday meetings (although I could attend only a fraction) to the PhD trip to Germany and Switzerland, working within FPE meant a lot to me and inspired me a lot. Thank you Marjan de Lange, for answering uncounted questions and always helping.

Among those that have taught me the most during this work were the people, that I was supposed to guide. I had the pleasure of supervising Sasja Antonisse, Dimitris Psinakis, Loes van Ooijen, Bas Ooteman and Melda Mutlu during their Master thesis and Fleur Crielaard and Nina Kosten during their Bachelor thesis. It was fun working with each of you and I thank you for your dedication and input. 
Further, I want to thank Matthijs de Winter from the University of Utrecht. We only met in person twice, but exchanged many e-mails and ideas. Thank you for your energy, expertise and input on taking electronmicroscopy pictures of the stationary phases. I entered a whole new world and value the experience.

This project was led by the ISPT, another organization that I am very grateful for. Through the ISPT I had the opportunity to see all this work in an industrial perspective, in addition to the academic perspective that university naturally tends to. In presenting my progress to the industrial partners of this project, and finding answers to all the questions that ensued, I gained a vantage point, that I would not want to miss. It ever highlighted the transition of fundamental towards applied science. I want to thank the industrial partners of Unilever (especially Hilde Wijngaard, Alphons Cambeen, and Hans-Gerd Janssen), of Royal Cosun (Johan van den Bergh and Edwin Poiesz), DSM (Nienke Hylkema, Jan Hein Willemsen, and Guilherme Ferreira) and FrieslandCampina, for their continued interest in this work and their support. I especially want to thank Johan van den Bergh for the interest in the SEM pictures and Matthew de Roode (Royal Cosun) for showing my paranymph Pina Fritz and me the inulin plant in Dinteloord on a very cold winter day.

Last but not least, I want to thank my family. I especially want to mention my mother, my father, and my mother in law. You came to help Sarahi, Clementina, and me, when we needed you the most. In the last months before handing in our theses, you came to stay at our house, help with the household chores and most importantly be with Clementina, while Sarahi and I worked till way too late. I am confident, that without your help, we would not have managed to finish the theses on time and together. Of course I want to thank my family for much more. Especially my parents, for supporting me throughout my studies and life, for the confidence and trust you placed in me, thank you.

To my daughter Clementina, I extend thanks for taking my mind off of work, like no-one else can. You do not know it yet, but you have helped me a great deal, just by being with us. Last but not least I want to thank my wife Sarahi. Writing this thesis was not always a pleasant experience, especially the final stretches. But the truth is, with you at my side, I enjoyed doing it and I am happy we are going through this together. Those nights, writing in the living room, are among the memories I will keep forever. 


\section{About the author}

Anton Schultze-Jena, was born on Sept. $14^{\text {th }} 1984$ in Hamburg, Germany. After primary school he attended the Walddörfer Gymnasium in Hamburg, to receive his high school diploma (Abitur) in 2004. His $11^{\text {th }}$ school year he spent in Odessa, Texas, USA, as an exchange student at the Odessa Career Center, living with a host family. Following high school, Anton did his (back then still mandatory) social service, opting out of military service. He worked 9 months at a daycare center for the elderly, specialized on patients suffering from dementia, involved in personal and hygienic care, transport, logistics and planning and executing daily activities. Following the 9 mandatory months, he continued to work for another 5 months and then spent the next 14 months travelling around the planet.

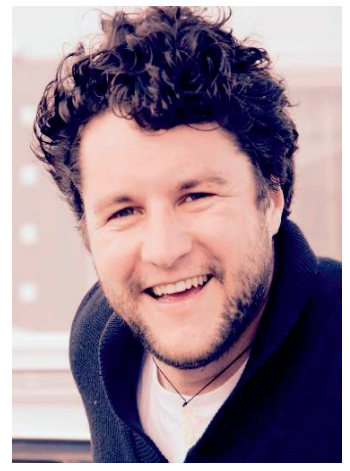

In 2007, Anton started studying Biotechnology at the University of Applied Sciences (HAW) in Hamburg, Germany, receiving his BSc. in 2012. During his time at the HAW, Anton worked as a teacher's aide in the department of organic chemistry and the department of fermentation and bioreactor technology. For the second half of 2010, Anton left Hamburg for an exchange semester in Brazil at the Universidade da Região de Joinville (Univille). Since his University in Hamburg told him (rather last minute), that credits received in Brazil would in all probability not be useable for his degree in Germany, Anton decided to change tracks slightly, and studied marine biology, while in Brazil. All courses were held in Portuguese. To finish his BSc. degree, Anton went to the Fraunhofer Institute for Marine Biotechnology (EMB) in Lübeck, Germany, where he worked with mammalian and fish cell cultures, developing a bioreactor concept for adherently growing cells. He stayed for one year, working as an intern (5 months), a thesis student (4 months) and finally as scientific assistant (3 months). In order to combine education with improving his Portuguese language skills and experiencing life in yet another country, Anton went on to study his MSc. in Biological Engineering at the Instituto Superior Técnico (IST) in Lisbon, Portugal in 2012. The majority of courses were held in Portuguese. He finished in 2014, writing his thesis on extended bed ion exchange chromatography at DSM in Delft, the Netherlands. In January 2015, Anton started his $\mathrm{PhD}$ at the department of Food Process Engineering at the University of Wageningen, the Netherlands. He was hired as an external PhD student, working with the group Functional Ingredients at TNO in Zeist, the Netherlands. In his last year, Functional Ingredients was merged with WUR Food and Biobased Research in Wageningen. Since April 2019 Anton is working for Wageningen Food and Biobased Research as a scientist. He lives with his wife and daughter in Wageningen and can be contacted at: anton@schultze-jena.de 


\section{List of publications}

A. Schultze-Jena, M.A. Boon, P.J.T. Bussmann, A.E.M. Janssen, A. van der Padt. The counterintuitive role of extra-column volume in the determination of column efficiency and scaling of chromatographic processes, Journal of Chromatography A, 1493 (2017) 49-56.

A. Schultze-Jena, M.A. Boon, D.A.M. de Winter, P.J.Th. Bussmann, A.E.M. Janssen, A. van der Padt. Submitted as: Predicting intraparticle diffusivity as function of stationary phase characteristics in preparative chromatography.

A. Schultze-Jena, M.A. Boon, R.C. Vroon, P.J.Th. Bussmann, A.E.M. Janssen, A. van der Padt. Submitted as: High viscosity preparative chromatography for food applications.

A. Schultze-Jena, M.A. Boon, R.C. Vroon, P.J.Th. Bussmann, A.E.M. Janssen, A. van der Padt. Submitted as: Elevated viscosities in a simulated moving bed using ion-exclusion chromatography: a case study on $\gamma$ aminobutyric acid recovery from tomato serum. 


\section{Overview of completed training activities}

\section{Discipline specific activities}

Conferences and Meetings

European Conference of Chemical Engineering (ECCE), Nice, (FR), 2015

Symposium on Preparative and Industrial Chromatography and Allied Techniques (SPICA), Vienna (AUT), 2016

Symposium on Preparative and Process Chromatography (PREP), Philadelphia (USA), 2017

\section{Courses}

Numerical methods for chemical engineers, OSPT, Eindhoven (NL), 2015

Microalgae process design, VLAG, Wageningen (NL), 2015

Sustainability analysis in food and biobased production, VLAG, Wageningen (NL), 2015

\section{General courses}

VLAG PhD week, VLAG, Baarlo (NL), 2015

Techniques for writing and presenting a scientific paper, VLAG, Wageningen (NL), 2016

Teaching and supervising thesis studentsEducational staff development, WUR, Wageningen (NL), 2015

Scientific publishing, WUR, Wageningen (NL), 2015

Effective behavior in professional surroundings, VLAG, Wageningen (NL), 2017

Interpersonal communication for PhD Students, VLAG, Wageningen (NL), 2015

Information Literacy for $\mathrm{PhD}$ including Endnote Intro, WUR Library, Wageningen (NL), 2017

Communication with the media and the general public, WGS, Wageningen (NL), 2017

Applied statistics for VLAG PhD, VLAG, Wageningen (NL), 2016

PhD workshop carousel, WGS, Wageningen (NL), 2015

PhD workshop carousel, WGS, Wageningen (NL), 2016

\section{Optional}

PhD study tour to Germany and Switzerland, FPE, 2016

PhD Symposium, WPC, Wageningen (NL), 2015

$\mathrm{PhD}$ Symposium, WPC, Wageningen (NL), 2016 
The research described in this thesis was financially supported by the Institute for Sustainable Process Technology ISPT. Financial support from Wageningen University \& Research for printing this thesis is gratefully acknowledged.

Cover design by Tania Erazo Ramos, printed by ProefschriftMaken. 



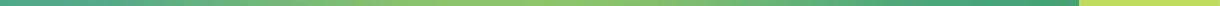

